\title{
Play it again, Sam! \\ An analysis of constructive replication in the organizational sciences
}

\author{
Tine Köhler \\ Department of Management and Marketing \\ Faculty of Business and Economics \\ The University of Melbourne \\ 198 Berkeley Street, Level 10 \\ Parkville, VIC 3010, Australia. \\ tkoehler@unimelb.edu.au \\ Jose M. Cortina \\ Department of Management and Entrepreneurship \\ School of Business \\ Virginia Commonwealth University \\ 301 W. Main Street, Box 844000 \\ Richmond, VA 23284 USA \\ jcortina@vcu.edu \\ josecortina.com
}

Acknowledgements: We wish to express our gratitude to Fred Oswald and two anonymous reviewers for their insightful comments. Work on the article was supported by the University of Melbourne's Faculty of Business and Economics.

Corresponding author: Tine Köhler, Department of Management and Marketing, Faculty of Business and Economics, The University of Melbourne, 198 Berkeley Street, Level 10, Parkville, VIC 3010, Australia. tkoehler@unimelb.edu.au 


\title{
Play it again, Sam! \\ An analysis of constructive replication in the organizational sciences
}

\author{
Tine Köhler \\ Department of Management and Marketing \\ Faculty of Business and Economics \\ The University of Melbourne \\ 198 Berkeley Street, Level 10 \\ Parkville, VIC 3010, Australia. \\ tkoehler@unimelb.edu.au \\ Jose M. Cortina \\ Department of Management and Entrepreneurship \\ School of Business \\ Virginia Commonwealth University \\ 301 W. Main Street, Box 844000 \\ Richmond, VA 23284 USA \\ jcortina@vcu.edu \\ josecortina.com
}

\begin{abstract}
Acknowledgements: We wish to express our gratitude to Fred Oswald and two anonymous reviewers for their insightful comments. Work on the article was supported by the University of Melbourne's Faculty of Business and Economics.

Corresponding author: Tine Köhler, Department of Management and Marketing, Faculty of Business and Economics, The University of Melbourne, 198 Berkeley Street, Level 10, Parkville, VIC 3010, Australia. tkoehler@unimelb.edu.au
\end{abstract}




\begin{abstract}
Replication is an essential part of any science, confirming or adjusting our understanding of the world through repeated exploration of a phenomenon of interest. While there has been an increased interest in the role of replication studies, there also exists skepticism regarding the need for more replication. Our empirical analysis of 406 recent studies that use the term 'replication' suggests that this criticism stems from a lack of appreciation of the different forms that replication can take, the prevalence (or lack thereof) of many of these forms, and the objectives that are met by one of the least common forms, constructive replication. As such, the purposes of our paper are 1) to explore the different forms that constructive replication can take and the objectives at which each can be directed, 2) to distinguish these forms from other forms of replication with which they are often confused, 3) to determine how common each form of replication is in our field, and 4) to provide concrete examples of different forms of constructiveness from published studies in order to pave the way towards more (and more useful) replications in the future.
\end{abstract}

Keywords: Replication, constructive replication, research design, empirical contribution 


\section{PLAY IT AGAIN, SAM! AN ANALYSIS OF CONSTRUCTIVE REPLICATION IN THE ORGANIZATIONAL SCIENCES}

Replication in science has received a great deal of attention in recent years. Calls for replication of findings and descriptions of different types of replication studies have appeared in such diverse fields as sociology (Adams, Ajrouch, Henderson, \& Heard, 2005), software engineering (Gomez, Juristo, \& Vegas, 2014), medicine (Lüdtke, 2008), economics (Mittelstaedt \& Zorn, 1984), and marketing (Easley, Madden, and Dunn, 2000) to name a few. The social and organizational sciences, though, have seen a particularly noteworthy increase in discussions of replication over the last few years. Perspectives on Psychological Science has devoted one special section (2012) and one special issue (2018) to the general subject, published another that deals with replication in a specific domain (i.e., priming, in the first issue of 2014), and published a third that includes treatment of related issues $(\# 3,2014)$. The Academy of Management created a new journal, Academy of Management Discoveries, that has as part of its mission the publication of replications of important findings. These and other sources include explanations of the importance of replication (e.g., Vazire, 2018; Anderson \& Maxwell, 2016; Bettis, Helfat, \& Shaver, 2016; Pashler \& Wagenmakers, 2012; Simons, 2014) and calls for change to the review process (e.g., Nosek \& Lakens, 2014).

Along the same lines, in each of the last few years, the annual conferences of the Society for Industrial and Organizational Psychology (SIOP), the Academy of Management (AOM), and the European Association of Work and Organizational Psychology (EAWOP) have contained several sessions related to replication in the organizational sciences. Furthermore, in 2016, SIOP founded the taskforce for robust and reliable research, which has produced a strategic report that highlights the importance of replications for creating robust research findings. Clearly, there is a 
great deal of energy around the idea that studies and analyses need to be repeated in one way or another to advance science.

At the same time, there remains skepticism about the existence of a replication crisis. Carpenter (2012), Pashler and Harris (2012), and others describe some of the objections to claims of a crisis, claims that the authors of the present paper have heard voiced in various panel discussions on the topic. As a specific example, a paper that received considerable attention in the popular press (e.g., Carey, 2016) was Gilbert, King, Pettigrew, and Wilson (2016). These authors sharply criticized the conclusions drawn by the Open Science Collaboration (2015; OSC) regarding replication. The claims of skeptics seem to fall into three categories. First, replication is quite common, so there is no pressing need for more of it. Second, replication studies are generally quite flattering to previous results. Third, where results fail to replicate, the failure can be attributed to the fact that the replication study differed from the original.

We argue that much of the skepticism regarding the need for more replication stems from a lack of appreciation of the different forms that replication can take, the prevalence (or lack) of many of these forms, and the objectives that are met by one of the less common forms constructive replication. The primary purpose of the present paper is to describe the various forms of replication and their targets, and to determine how common each form is. This allows us to ascertain the scientific needs (e.g., the need to quantify sampling error or to understand the degree to which findings are due to methodological characteristics) that are and are not being met by current replication practices. In particular, our examination of published journal articles in three top tier journals reveals that the most useful form of replication, constructive replication, is one of the least common forms currently employed by researchers. Furthermore, the most useful form of constructive replication, the independent comprehensive variety, is almost 
unheard of. In short, our field contains a great many of the less useful forms of replication, and very few of the most useful forms. Consequently, although we may not have a crisis in the form of an insufficient number of replications, we do seem to have a crisis regarding the usefulness of these replications as a whole.

We begin by providing an overview of prior definitions of replication to derive one integrative definition and to distinguish replication from reproducibility and generalizability. Next, we distinguish the different forms that replication can take and describe the functions that these forms can serve. We then report findings from an empirical examination of 406 recent studies in three top journals that claim to conduct some form of replication. The purposes of this examination are to determine what authors mean when they use the term 'replication', and to understand how common the different forms of replication are. Finally, we provide guidance for authors, reviewers, and editors in an attempt to cultivate a culture of constructive replication in our field.

\section{Distinguishing among replication, reproducibility, and generalizability}

As was mentioned earlier, one reason for disagreement regarding the replicability of findings in our field and the frequency with which such tests are conducted seems to be that different people use certain terms in different ways. One can find reference to reproducibility, literal replication, direct replication, exact replication, strict replication, conceptual replication, partial replication, quasireplication, operational replication, instrumental replication, constructive replication, negative replication, positive replication, internal replication, external replication, differentiated replication, dependent replication, independent replication, and transferability, to name a few (Benson \& Borrego, 2015; Gomez et al., 2014; Kelly, 2006; McElreath \& Smaldino, 2015). Gomez et al. (2014) reviewed 20 papers across many disciplines that had attempted to 
distinguish among different replication types and found that, “...there are no standard intra- or interdisciplinary terms to refer to different replication types (2014: 1035)." In and of itself, the existence of so many terms is not a problem given the complexity of the topic. The problem is that some of these terms are interchangeable while others are used interchangeably when they shouldn't be. Even more importantly, there are critical distinctions that are not reflected in the current set of terms and definitions.

We begin, therefore, by distinguishing among the umbrella terms to be used in this paper. We intend for our definitions to be as consistent with previous work on the topic as possible, but we depart somewhat from previous work when doing so allows us to maintain important distinctions.

Asendorpf and 15 coauthors from around the world (Asendorpf et al., 2013) define reproducibility in a way that makes clear its distinctiveness. Reproducibility involves a single dataset being analyzed more than once, either by a single researcher (which we label dependent reproducibility) or by different researchers (independent reproducibility; see Figure 1a for an illustration). Let us suppose two researchers, A and B. Independent reproducibility exists if Researcher B reanalyzes the data of Researcher A and arrives at the same results and presumably conclusions (Asendorpf et al., 2013). This is essentially Gleser's (1996) first law of applied statistical analysis, which he defines as, "Two individuals using the same method on the same data should arrive at the same conclusions (1996: 220).” Gleser goes on to say that adherence to this law, “...is clearly fundamental to scientific reasoning for how else can scientists check each other's work?" (1996: 220). How indeed? Of course, if a science is to be self-correcting, then there must be someone to do the checking (see the last two of Merton's, 1942/1973, conditions for a self-correcting science). 
In any case, reproducibility involves a single data set, and the issues that are the target of a reproducibility study include the statistical competence of the original researcher as well as his or her thoroughness in reporting data analytic methods and results (see Aguinis, Ramani, \& Abduljader, 2018 for detailed examples of these sorts of targets). Asendorpf et al. (2013) describe reproducibility as a precondition for more complex criteria such as replicability and generalizability. Similarly, Aguinis et al. (2018) describe results reproducibility as necessary but insufficient for inferential reproducibility. Although the focus of the current paper is on replication rather than reproducibility, it is worth noting that such studies can be quite useful. Edwards and Harrison (1993) and Hollenbeck, DeRue, and Mannor (2006) offer good examples. Concerns over reproducibility have also led to many article retractions in recent years. That said, such studies are rare in our field.

Whereas reproducibility refers to a single data collection or study, replication involves the study of a phenomenon (e.g., the relationship between two variables) being conducted more than once, either by the same set of authors (dependent replication) or by different sets of authors (independent replication) (see Figure $1 \mathrm{~b}$ for an illustration). This implies that a replication does not necessarily have to lead to the same conclusion. However, if a researcher stipulates that findings were replicated, then they did have to come to the same conclusion as previous research. Let us assume again two researchers, A and B. A result or set of results is independently replicated if, in some way, Researcher B examines the same phenomenon that was examined by Researcher A and arrives at the same conclusions. The replication can be an attempt to repeat the original test exactly or it can include differences in design (e.g., superior dependent variable measures, experimental versus observational) from the original test. In fact, some replications may target design characteristics under which findings do not replicate. A 
good example for this is the paper by Schultze, Pfeiffer, and Schulz-Hardt (2012), which we describe in more detail later.

Replication might be pursued in an attempt to identify any number of sources of betweenstudy variability. Among the most common are chance, experimenter competence, experimenter motives, reporting accuracy and completeness, predictor operationalization, criterion operationalization, task fidelity, and sample representativeness. One of the central tenets of the present paper is that different replication designs target different sources of variability, and the choice of replication design should be driven by the source of variability that one wishes to evaluate. For example, a literal replication, in which the design of the replication study mirrors exactly the design of the original study, is primarily concerned with variability due to sampling error, although it can also target factors such as transparency. Other forms of replication, such as those that change aspects of the research design, may be concerned with variability due to differences in measurement error. We further explore different forms of replications and their targeted sources of variability later in the paper. In any case, the variability sources that are the target of replication as we define it all have two things in common. First, they represent potential moderators of one or more linkages in the model. Second, they represent factors that are potentially relevant to findings but are not really part of the model being tested. In other words, they represent methodological moderators.

Finally, there is generalizability. Generalizability has been defined in many different ways, both within and outside of discussions of replication. Many of these overlap in one way or another with definitions of replication. The definition of generalizability used by Asendorpf et al. (2013) revolves around the notion that findings should not depend on a particular type of unmeasured variable. These unmeasured variables could be study characteristics, in which case 
generalizability seems conflated with replication. The unmeasured variables can also be of the sort that might reasonably appear as substantive moderators in a path diagram of a conceptual model (Asendorpf et al. use subject gender as an example).

In order to make the distinction between replication and generalizability clear, we define generalizability as lack of variation in findings across studies that differ on one or more substantive moderators. Given this definition, one should have few concerns about the degree to which generalizability studies exist in our field. The number of studies that contain such substantive moderators has been growing steadily for decades (Cortina, 1993; Aguinis, Edwards, $\&$ Bradley, 2016). Thus, we restrict our attention in this paper to replication, and we concern ourselves with the characteristics and targets of such studies rather than the criteria that might be used to conclude replicability (i.e., finding the same results as previous studies of the same phenomenon) (cf. Anderson \& Maxwell, 2016). It is worth noting, however, that when authors claim to be "replicating and extending" previous work, they are often talking not so much about replication but about generalizability.

\section{Dimensions of Replication Studies: Constructiveness and Researcher Independence}

There are many different forms that replication studies can take (see Table 1 for definitions, descriptions, and examples). In this section, we discuss two core dimensionsresearcher independence and constructiveness - on which replication studies can vary. The first of these is fairly well-understood (though seldom recognized), so we offer only a brief description. The second is, as our empirical review shows, much less well understood and requires more discussion. In any case, it is important to understand not only differences in the procedures through which replication is established but also differences in the specific concerns 
FRAMEWORK FOR REPLICATION

with previous findings that researchers might address by conducting a replication study of a particular kind.

\section{Insert Table 1 here}

Researcher Independence. The first general dimension on which replication studies differ is researcher independence. A replication study is independent if it is conducted by an entirely different set of researchers from the original study. At the other end of the scale would be a study conducted by the same set of researchers who conducted the original. It is also possible to have a semi-independent study if a subset of the researchers who conducted the original study joins the team that conducted the replication study.

Those who are skeptical of the need for more replication point out that multi-study papers are increasingly common in the organizational sciences. This is true, and we consider this trend to be a good one. However, because Study 2 in a multi-study paper is conducted by the same researchers who conducted Study 1, it does not represent an independent test. The design knowledge that led to the design of study 1 will also be relied upon in designing study 2 . Furthermore, the original team's incentive to find support for their hypotheses is just as strong in study 2 as it is in study 1, which again may affect their design choices or statistical analyses. Without independent corroboration, science cannot be self-correcting (Vazire, 2018). The overarching issues that researcher independence targets are competence and the effect of incentives to obtain findings that are favorable vis-à-vis hypotheses. 
Constructiveness. This term is familiar to all of us, but it is clear from our review that much of our field has only a vague notion of what a constructive study would look like. Thus, a deconstruction (as it were) of the term might be of some use.

Replication studies can differ in the degree to which they simply repeat versus improve upon previous studies. The most common citation for definitions of constructive replication is Lykken (1968), who said:

To obtain an ideal constructive replication, one would provide a competent investigator with nothing more than [original emphasis] a clear statement of the empirical "fact" which the first author would claim to have established [...] and then let the replicator formulate his own methods of sampling, measurement, and data analysis (p.156).

Implied in this definition is the notion that the replicator would choose the best possible design. We suspect, however, that because this is only implied, many have been confused about the true nature of constructive replication. We therefore define constructiveness more concretely: A study is constructive if it retains all of the virtues of the previous study but includes at least one methodological improvement, thus allowing a test of hypotheses that is clearly superior.

We intend for this definition to be consistent with the spirit of Lykken's (1968) definition of constructive replication while at the same time offering a more specific reference to quality of evidence (see Fiedler, 2018 for a treatment of the evidentiary quantity/quality distinction). For example, a study that repeats a previous study but uses a higher fidelity task is constructive. If it used a higher fidelity task and a less representative sample, it would not be constructive. As we show later, this latter sort of study is increasingly common in our field, and it is often labeled 
'constructive replication.' This fact may explain why some believe that there is no replication crisis. We term this type of replication confounded.

It should be noted that, although there are design attributes that are universally virtuous (e.g., task fidelity, presence of manipulation checks), others may or may not be considered part of a constructive replication depending on the question being asked. For example, supervisor ratings of performance may be superior to, say, peer ratings of performance in some circumstances. If, however, the dimension of performance under consideration is one to which supervisors are unlikely to be privy (e.g., undermining of supervisor), then supervisor ratings may be inappropriate. To some degree, the design attributes that comprise the ideal design vary from question to question.

In any case, replication studies can vary in the degree to which they are constructive. For example, a replication study may attempt to resolve all of the most important weaknesses of previous tests. We would characterize such a study as a comprehensive constructive replication. At the other end of the spectrum would be replication studies that address one or more minor flaws in previous work but leave all major methodological shortcomings of the previous study unresolved. We might label these incremental constructive replications. Although the "comprehensive" and "incremental" bars represented in these definitions may seem rather high and low respectively, they have the advantage of being relatively easy to judge. Relatedly, they represent opposite ends of the constructiveness spectrum: incremental studies address none of the major shortcomings, while comprehensive studies address all of them.

In between the comprehensive and incremental constructive replication would be the study that attempts to incorporate improvements to some, but not all, of the important flaws in previous research designs. We call this a substantial constructive replication. As we show later, 
neither comprehensive nor substantial constructive replications are at all common. The bar that is set by our definition of comprehensive constructive replication is indeed high, but a study needn't be comprehensive in order to make a tremendous contribution. In our opinion, the field would be far more successful in meeting Merton's (1973) criteria for a self-correcting science if it contained more replications that were "merely" substantial.

These distinctions are, of course, a matter of judgment, although the studies at either extreme are easy to identify. Generally, though, we label as constructive any study that is superior to previous studies in at least one way for answering the research questions at hand while at the same time not being inferior to previous studies in any way. These improvements may include more valid measures, more critical control variables, a more realistic task, or a more representative or relevant sample, just to name a few.

As an example of a comprehensive constructive replication, consider Eden and Shani's (1982) study as a gold standard upon which any researcher would be hard-pressed to improve. The Introduction of Eden and Shani (1982) is devoted almost entirely to the limitations of previous attempts to test the Pygmalion effect, the vast majority of which involved weak expectancy manipulations in elementary school classrooms. The authors conducted a field experiment in a high-stakes environment (i.e., a combat command course in the Israeli military) with measures of student performance and instructor behavior, and they were able to control for student cognitive ability. Expectancy was manipulated by giving instructors an evaluation of “command potential" (CP) which was based on, "psychological test scores, sociometric data from the previous course, and ratings by previous commanders $[\ldots]$ course grades predict trainee CP in 95\% of the cases" (p. 197). Eden and Shani's (1982) study represented an improvement over previous efforts in almost every respect including sample (working adults), setting (high- 
stakes), design (“after only” randomized block experimental), independent variable operationalization (evaluation based on multiple identifiable sources of information), and dependent variable operationalization (objective and subjective, trainee and instructor). Because it retained the virtues of previous tests but none of their major flaws, Eden and Shani (1982)'s study can be described as comprehensive constructive replication.

In order to understand the implications of constructiveness, it might be instructive to consider studies that lack constructiveness. One of the criticisms levied against the OSC was that its studies did not repeat exactly the procedures used in the original studies. Borrowing a term from Lykken (1968) and Kelly, Chase, and Tucker (1979), we might say that Gilbert et al. (2016) criticized the studies in the OSC for not being literal replications, also referred to as direct replication (e.g., Simons, 2014) and exact replication (Stroebe \& Strack, 2014). Although such studies certainly have value, the conclusions that can be drawn from them are limited. The targets of literal replication depend on whether the replication is conducted by the same researchers who conducted the original study. If so, that is, if the literal replication is dependent, then the only target of the replication is sampling error. If the literal replication is independent, then it can also target experimenter competence regarding research design, experimenter malfeasance, or reporting accuracy. Because they repeat the designs of previous tests, literal replications do not tell us anything about the effect of design characteristics on tests of hypotheses. This limitation is critical wherever particular design flaws are common (such as using a dominant, but flawed, measure or using observational designs to draw causal inferences). If such common flaws are merely repeated, the deductive part of our science cannot function (Wagenmakers, Dutilh, \& Sarafoglou, 2018). 
The low end of the constructiveness dimension also contains a more nebulous, but very common, category that we label quasi-random. In discussing replication more generally, Asendorpf et al. (2013) point out that although we usually think of design characteristics as fixed factors, one could also conceptualize them as random. In fact, Asendorpf et al. (2013) actually define replicability as, "the finding can be obtained with other random samples drawn from a multidimensional space that captures the most important facets of the research design (2013: 109)." We chose not to employ the Asendorpf definition of replicability because study characteristics are not, in fact, sampled randomly. There are many factors that drive design choices. If the factor that drives a given design choice is desire for quality, then the study would fall into the constructive category. If instead factors such as familiarity, convenience, or availability are responsible for design choices, then the study would fall into the quasi-random category.

A quasi-random replication study is one that differs from the original but that isn't clearly better or worse for the research questions being asked. Thus, the quasi-random replication is not literal. But neither is it constructive because these differences do not necessarily reflect improvements upon the previous study. The distinctiveness of a quasi-random study is due to factors other than design quality. If one were to sift through the studies included in the meta-analysis of a particular relationship, one would observe differences in a variety of design characteristics (e.g., the effect of different adaptations of a specific survey as per Mackey, Frieder, Brees, \& Martinko, 2017). Variability in a design characteristic allows one to determine whether that characteristic is relevant for the relationship in question. While this is useful, it leaves open questions regarding the effects that would be observed if superior characteristics were applied. 
In response to calls for more replication, one often hears the rebuttal that if we don't have enough replications, then how can we possibly have so many meta-analyses? The answer lies in recognition of the fact that quasi-random replications, particularly of bivariate correlations among global observational variables, are very common indeed. In fact, as we show later, we found that roughly $52 \%$ (i.e., 211 out of 406 studies) of the studies claiming to have conducted a replication study are quasi-random replication studies. When social scientists such as Schwartz claim that robustness concerns are overblown (Carpenter, 2012), they cite as evidence the presence of "conceptual replications", but most of these are quasi-random". While such replications are useful for some purposes, they are less useful for others. If we borrow Whewell's metaphor of the scientific staircase (1840), we might say that whereas other forms of replication take us several steps toward deduction, quasi-random replication only gains us a single step. We contend that those who call for more replications are not calling for more quasi-random replications but rather for more constructive replication studies, and perhaps more literal studies as well, because there are so few of them and because they address important issues that are not addressed by quasi-random replications.

In the next section, we describe an empirical review of published papers in AMJ, JAP, and JOM. The purpose of this review was to assess how frequently researchers employ the different forms of replication we just described and examine the utility of their application in the reviewed papers.

AN EMPIRICAL REVIEW OF THE FREQUENCY OF DIFFERENT FORMS OF REPLICATION Selection of studies for review of current replication practices 
We began with a search of papers in three top-tier journals, Academy of Management Journal (AMJ), Journal of Applied Psychology (JAP), and Journal of Management (JOM), from 2007-2017 with replicat* in the title and/or abstract. We then expanded the search to full-text mentions of replicat* for papers published between 2012 and $2017^{2}$. We decided to limit the fulltext search to this timeframe to determine the most recent use of the term "replication" in published work, but also to keep the coding process manageable. Between 2007 and 2017, we found 77 papers with replicat* in the abstract or title. Between 2012 and 2017, we found 719 papers that contained replicat* in the text. We recognize there are papers that contain replications without ever using the term, and that there were papers published prior to 2012 and 2007 , respectively, that would have used the term. Our goal was not to produce an exhaustive review of the journals but rather to understand how the term "replication" and its derivatives are currently being used and how common different forms of replication are.

From the papers turned up in these searches, we excluded papers that did not actually collect any data (e.g., editorials, reviews, or author guidelines), papers that only used the term replication somewhere in their reference list, papers in which authors only stated that their work needs future replication but they themselves didn't claim to have conducted a replication, and papers that used the word replication in a sense that was not captured by our definitions. For example, some authors used the term in the sense that they recreated materials in a way to reflect a particular context (e.g., a scenario replicates a real work experience). In a number of papers, authors used the term replication to refer to iterations in bootstrapping procedures or permutations in Monte Carlo simulations. Given that our definition of replication relies on collecting new data, not artificially generating data, we excluded these papers from our analysis. 
Amongst the papers meeting our inclusion criteria, many papers provided more than one replication study. We counted each independent data collection effort targeted at retesting a hypothesis or set of hypotheses as a separate replication study, following common conventions for coding independent samples. Our final dataset included 508 independent studies or samples (References for these studies in the online Supplementary Materials).

\section{Coding of replication studies}

We coded each putative replication for a variety of characteristics. As a first step, we coded where in the paper some version of the term replicat* was used, i.e., in the abstract or in the main body of the article. We then coded for the meaning with which authors employed the term. Because we had noted that the term replication and related terms were used in either one of two particular ways, we distinguished between usage of the term to mean that the study was repeated in some way and usage to communicate the notion that the same result was found in multiple studies. Sample wording includes "in our study, we replicated previous experiments by testing..." or "our study replicated previous findings."

We also coded for whether any sort of constructive language was used. For example, Hanek, Garcia, and Tor (2016: 1122), state in their abstract: "Studies 2a and 2b replicate the effect with real behavioral decisions in different domains [emphasis added]." As another example, Thau, Derfler-Rozin, Pitesa, Mitchell, and Pillutla (2015: 98) highlight how their second study improves over their first study: "A 2-wave field study conducted among a diverse sample of employees working in groups (Study 1) and a constructive replication using a laboratory experiment (Study 2) provided converging evidence for the theory."

We then coded for the level of independence. If a study was conducted or a data analysis rerun by the same researchers as the original, it was labeled dependent. If a study was conducted 
or a data analysis rerun by different researchers than the original, it was labeled independent. If the research team of the new study or data analysis retained one or more researchers from the team of the original study, it was labeled semi-independent. Furthermore, we coded for whether the phenomenon being replicated was a single relationship (e.g., a specific correlation) or multiple relationships (i.e., a model of some sort or multiple hypotheses).

Finally, we coded for the type of replication employed in the study, using the categories that we described earlier. This was the only code that required judgment. We therefore made the coding as concrete as possible. For judging constructiveness, if a study or data analysis retained the characteristics of the original research design but improved upon it in some way, it was coded as constructive. We judged a study to be incrementally constructive if it corrected at least one minor flaw in the original research design but kept all major flaws. A study was coded as substantially constructive if it addressed at least one major shortcoming of the previous research design, but it retained other major shortcomings. For a study to be coded as comprehensively constructive, the research design of the replication study had to resolve all major shortcomings of the original study's research design.

Examples for major shortcomings in research design would be reliance on potentially unemployed student populations when the phenomenon of interest was clearly employment related, reliance on self-reported job performance, use of all self-report data (when other sources of information would have been better), or use of low fidelity, low stakes tasks when the phenomenon of interest is more likely to emerge in a high-fidelity setting. As these examples indicate, whether or not a specific research design choice represents a major shortcoming depends in part on the research question that is being addressed. For example, student populations can be entirely appropriate in studies that address research questions related to the 
value of internships, the experiences of first-time job seekers, or the effectiveness of certain learning approaches. Similarly, self-report data are entirely appropriate when researchers want to explore perceptions, attitudes, and beliefs of the individual. As such, the degree of constructiveness needs to be determined vis-à-vis the chosen research question and the intended conclusions to be drawn from the data collection.

If a study or data analysis differed from the original research design without clearly improving the design, then it was labeled quasi-random. For example, the original study might have used a particular measure of an attitude while the replication study used another. Or perhaps the original study used white collar employees and the replication study used blue collar employees to answer a question for which the white collar- blue collar distinction is unimportant.

Because our field contains many studies or data analyses that are superior to previous efforts in some ways but inferior in others, we created a category that we labeled 'confounded replication.’ For example, in some papers, researchers might have used an organizational employee sample in study 1 but used self-report for measuring all variables. In study 2 , they might have used an experimental design, thus addressing the problem of common method variance and strengthening causal inference, but relied upon a sample of potentially unemployed undergraduates to answer their employment context research question. In this case, the appropriateness of the sample for the research question is inferior, but the ability to draw causal inferences is superior. Such a study would have been labeled as confounded. As we discuss later, although confounded replications are increasingly common, their value is not altogether clear.

If the study design in the replication was an exact repetition of the design in the original study, then it was labeled as literal. Finally, if the purpose of the study was to test substantive moderators for a relationship, it was coded as generalizability. 
Coder Agreement. We first examined all relevant published papers in the time frames outlined above in JAP. The search and coding of the word replicat* in the abstract and title was initially completed by the second author of the paper, employing the coding scheme outlined above to the 58 studies that emerged from this search. The first author of the paper then also coded all of these studies independently. After this, the two co-authors discussed their coding to reach $100 \%$ agreement and resolve any ambiguities in the coding scheme. Subsequently, the first author conducted the full-text search of the word replicat* in JAP and coded all of the obtained papers. Throughout the coding process, she kept notes in the coding file that assisted in discussing cases with the second co-author during their coding meetings. To assess coder agreement on the full-text search, we randomly selected 10 studies for the second author to code independently. Initial coder agreement for these 10 studies was $93.75 \%$. Disagreements were discussed and resolved. The first author then coded papers published in AMJ and JOM. She identified 74 cases about which there was some uncertainty, mainly regarding the distinction between incremental and substantial constructive replication and the distinction between confounded and quasi-random replication. For both distinctions, the problem lay in the fact that the virtues of many of these replications were relative rather than absolute. For example, there were several replications that retained several major flaws of the replicated study but that corrected one other major flaw. Although it seemed strange to label such studies "substantial constructive", this was the only way to keep this code relatively objective (i.e., a replication is substantial if it corrects at least one major flaw without introducing any new ones). For these and other distinctions, we stuck with the approach that others would find easiest to replicate.

After discussing the general issues that had given the first author pause, these 74 cases were independently coded by the second co-author, and discrepancies were discussed to reach 
$100 \%$ agreement. Initial agreement on the coding choices was $97.5 \%$. Finally, we further independently coded 23 studies at the end of our coding to determine if our coder agreement remained high. Initial agreement was $96.44 \%$, and final discussion resolved all disagreements. Altogether, we independently assessed 165 out of 508 (i.e., 32.5\%) of studies in the data set.

\section{RESULTS}

In the following, we present the frequencies of different types of studies found in our review. In addition, to illustrate the different forms of replications represented in published work, we provide some concrete examples of different types of replication studies to help authors of future work assess whether their planned research design includes more or less useful forms of replication.

\section{Insert Table 2 and Table 3 here}

Out of the 508 studies we coded (complete coding table can be found in the online Supplementary Materials), 406 represented different forms of replication studies, 35 (6.9\%) were in fact generalizability studies, in which the authors tested substantive moderators, 64 were reproducibility studies $(12.6 \%), 2$ were studies with repeated measures but not replications, 1 was a first-time hypothesis test. We did not code the latter four categories of studies further as they did not match our definition for replication. 319 (78.6\%) out of the 406 coded studies were dependent, i.e., conducted by the same authors of the original study, 79 (19.5\%) were independent, and $8(2 \%)$ were semi-independent, i.e., where at least one co-author on the replication study was also a co-author of the original study (see Table 2 for a summary and Table 3 for results presented separately for each journal). 5 out of 319 dependent studies were 
published across separate papers, the rest were published within the same paper (i.e., multi-study, multi-sample, or re-analysis). Thus, there appears to be a strong preference for replicating one's own work, rather than replicating the work of others. One root cause for this may be the emphasis on novelty vis-a-vis the work of others. Another root cause may be authors are concerned that replication attempts (especially those that do not find the same results) may be assessed negatively by reviewers. We discuss both root causes in more depth later in the paper.

\section{Quasi-random replication studies}

The most common form of replication study is the quasi-random replication $(52 \%, 211$ out of 406 studies). Amongst those, 64\% (135 out of 211) are dependent, 32.2\% (68 out of 211) are independent, and 3.8\% (8 out of 211) are semi-independent. Dependent quasi-random replications are the single most frequent overall form of replication study we found $(33.3 \%, 135$ out of 406 studies). As alluded to earlier, the prevalence of quasi-random replication studies may largely be responsible for the existing perception that plenty of replication studies already exist in the organizational sciences.

As an example of dependent quasi-random replication, Venkataramani, Labianca, and Grosser (2013) conducted a Study 2 to replicate their Study 1. Study 2 data came from a product development firm instead of a food and animal science organization, which seems neither more nor less appropriate for the study of valence of network ties and organizational attachment. Study 2 used many of the same measures but used different measures of affective commitment and advice ties. These different measures do not seem to be better or worse than those used in Study 1, and the authors make no such claims. Thus, Study 2 differs from Study 1, but not in a way that makes it better or worse for the questions being addressed. It is neither constructive nor confounded, but quasi-random. 
As an example of independent quasi-random replication, consider the primary studies in the meta-analysis by Van Iddekinge, Aguinis, Mackey, and DeOrtentiis (2018). Among other things, the primary studies in this meta-analysis of the effects of cognitive ability and motivation on performance varied with regard to whether they were conducted in civilian versus military settings. Clearly, this sample difference means that the civilian studies are not literal replications of the military studies. It isn't clear, however, that one of these settings is superior to the other for purposes of testing ability by motivation interactions. As a result, with all else being equal, the civilian studies should be characterized as quasi-random replications of the military studies (or vice versa, which is another characteristic of the quasi random replication) rather than constructive replications.

Replications for the primary studies in this meta-analysis would be constructive if they included, for example, measures of motivation not tainted by intentional distortion and selfdeception. However, Van Iddekinge et al. (2018) were unable to compare constructive replications to more typical studies because so few primary studies contained measures of motivation that were not self-report (personal communication, H. Aguinis, June 5, 2017).

Because quasi-random replication isn't constructive, it doesn't address issues of design appropriateness and tells us little about experimenter competence/motives. Instead, it concerns itself mainly with issues of sampling error (e.g., error associated with sampling of subject pools, of predictor scales, of task type, etc.) and perhaps reporting thoroughness. The generation of a number of studies such as this makes meta-analytic estimation of population effect size and variance due to sampling error possible, although the conclusions drawn from such metaanalyses should be restricted to a certain level of design quality. With this type of replication, the researcher can also discover something about the influence of certain study design characteristics 
on the relationship in question. Such discovery is, however, limited to the design characteristics that are commonly used, while many other characteristics (i.e., those that are superior but more difficult to implement) occur too infrequently to be examined. ${ }^{3}$

\section{Constructive replication studies}

Although quasi-random studies are by far the most common, the second most common group of replication studies are the constructive replication studies $(23.9 \%, 97$ out of 406 studies). Amongst these, 66\% (64 out of 97) are dependent incremental constructive, and 7.2\% (7 out of 97) are independent incremental constructive. As such, roughly $73.2 \%$ of all constructive replication studies improve only slightly upon the research design of the original study. We found $25(25.8 \%)$ dependent substantial constructive replication studies, that is, studies in which the authors solved at least one but not all of the major shortcomings in prior studies. It is somewhat telling that we found no studies whatsoever that substantially or comprehensively improved a prior study of a separate team of authors. In addition, we only found one dependent comprehensive constructive replication study, i.e., one study that addressed the major shortcomings of the previous study conducted by the same authors. Together that means, that only 26 out of all 406 studies (6.4\%) improve at least one major flaw in the previous research design without making other aspects of the design worse.

Garcia, Restubog, Kiewitz, Scott, and Tang (2014) test the relationship between supervisor's history of family aggression and abusive supervision. They designed four studies to test their hypotheses. While study 2 was an incremental constructive replication over study 1, in which the authors retested Hypothesis 1 with an implicit measure of cognitions, study 3 was a comprehensive constructive replication of studies 1 and 2. As the authors describe: 
In Study 3, we obtained parent ratings of family aggression history to minimize response distortion and memory recall problems associated with retrospective accounts (Hardt \& Rutter, 2004). We included a temporal element in Studies 3 and 4 to help reduce concerns associated with common method variance (Podsakoff, MacKenzie, \& Podsakoff, 2012). To rule out alternative explanations for our findings, we included previously established antecedents of abusive supervision as control variables in Studies 2-4 (p. 886).

Furthermore, the authors didn't confound their replication by going back to a self-report measure of cognitions. While this is a good example of what a comprehensive constructive replication should look like, it also throws into sharp relief the fact that, although constructive replications do exist in our field, they are rarely comprehensive.

Less uncommon are dependent substantial constructive replications. For example, in their 2015 study on the link between team cohesion and performance, Mathieu, Kukenberger, D'Innocenzo, and Reilly conducted two primary studies with student teams. In the first study, the authors observed student teams over the course of a 10-week business simulation, during which they measured team cohesion at three points in time. GPA was measured pre-simulation to approximate academic competence, and performance was measured via the outcome statistics of the simulation at the end of each performance round. Furthermore, shared leadership was measured at one time during the second survey. Finding that cohesion correlated positively with performance and vice versa and that shared leadership contributed positively to team cohesion, the authors attempted to replicate their findings, using a larger number of student teams (to combat sampling error issues) and assessing shared leadership as an emergent team state (i.e., as developing dynamically over time) instead of as a stable between-team variable. This replication study is an example of a substantial constructive replication because it included a nontrivial 
improvement upon the measurement of shared leadership (i.e., one that captures its emergent nature) and upon the modest sample size of study 1 . As a result, the constructive replication in Mathieu et al. (2015) targeted predictor validity and sampling error. Furthermore, given that the authors' original study design was quite strong, a second study in which any improvement at all was made would be valuable.

While we did not find among the 406 studies any one study that could be considered independent substantial or comprehensive constructive, we found a multi-study paper that we consider to be an independent comprehensive constructive replication.

Schultze et al. (2012) explored the effect of biased information processing on the amount of reinvestment in decision-making tasks. In their first experiment, the authors attempted to replicate the findings of biased information search in Conlon and Parks (1987). Having speculated that one particular piece of information in the item pool used by Conlon and Parks might have swayed findings, they generated a more appropriate pool of items and compared findings obtained with the old and the new item pool. The authors found that they could replicate Conlon and Park's findings when using the old item pool, but not when using the new one. Schultze et al. (2012) then conducted a second experiment in which the manipulation was strengthened, the task was made more realistic in several ways, and a potential confound was manipulated. Using this improved design, the authors could not find support for information search bias in the second experiment. Expanding on these findings, in experiment 3, the authors added an information evaluation phase to test whether biased information processing is in fact due to biased evaluation of information, rather than biased information search. Finding support for biased information evaluation (rather than information search), the authors conducted a fourth experiment in which they manipulated a putative causal variable that had been measured 
in the previous studies. Thus, through a series of experiments, they were able to improve upon the most important features of previous designs. As a result, they distinguished the actual facets of information processing bias from those that were merely artifacts of design flaws. Unlike Eden and Shani (1982), the comprehensiveness of Schultze et al.'s (2012) constructive replication resides in the combination of the four studies. None of the 4 studies alone addressed a substantial number of the important flaws of previous tests, but as a set they do.

\section{Confounded replication studies}

71 out of 406 studies (17.5\%) were confounded replications, out of which 69 (97.2\%) were dependent and $2(2.8 \%)$ were independent. As we explained above, the value of these replication studies is unclear as they do not allow one to isolate the effects of the improvement. For example, consider the study by Chen, Chen, and Sheldon (2016). Study 1 was a longitudinal survey with an objective measure of unethical pro-organizational behavior (UPB). The inclusion of an objective measure of UPB is particularly impressive. The authors noted two limitations of this study, namely, that it included only a single measure of UPB and that it did not include a measure of interorganizational competition. Study 2 included such a measure and included a broader measure of UPB. On the other hand, whereas Study 1 involved an objective measure of UPB, Study 2 measured only willingness to engage in UPB. Thus, although two limitations of Study 1 were improved upon, one of its most virtuous attributes (an objective measure of deviance) was lost. Hypotheses were once again supported in Study 2. Unfortunately, it is impossible to say whether the results of Study 2 represent replication in a superior design or in an inferior one. Should we conclude that the results of study 1 replicate to a broad range of UPB, or merely that they replicate for hypothetical behavior? 
In order to clarify the problem inherent in confounded replications, let us suppose that the model being tested in a given study is $\mathrm{X} \rightarrow \mathrm{Y}$. Let us also suppose that the effect is weaker in high quality studies than in low quality ones. Now, imagine a Study 1 that is high in quality on design characteristic A and low in quality in design characteristic B, and that Study 1 found a regression weight of $b_{x y}=.30$. Suppose that the purpose of Study 2 is to examine the possibility that the reason for the substantial $b_{x y}$ in Study 1 was a low-quality design characteristic B. Thus, Study 2 is designed to be high in quality on characteristic B. Unfortunately, design characteristic A could not be repeated in Study 2, and a lower quality version had to be used instead. If we find $b_{x y}=.30$ in Study 2, we have no way of knowing if this should be interpreted as vindicating the finding from Study 1 in spite of its flawed design characteristic B, as supporting the notion that $b_{x y}$ in Study 2 was an artifact of a low-quality design characteristic A, or both.

It should also be noted that, although confounded replications may be overvalued, they can be useful. Consider the replications in Dillon, Tinsley, Madsen, and Rogers (2016). Study 1 involved archival data from 30 actual NASA missions administered by the JPL. Clearly their primary predictors, safety climate and project stakes, could not be manipulated for actual NASA missions. Therefore, studies 2-4 were vignette studies with NASA trainees. Vignettes are necessarily decontextualized, and there were few consequences for the actions of the trainees. On the other hand, participants were randomly assigned to condition. Although the design is clearly confounded, it is difficult to imagine a data collection opportunity in which there could be random assignment to actual safety climate and actual project stakes conditions. The fact remains, however, that it is impossible to tease apart the effects of the improvements from the effects of the compromises. 
As can be seen in Table 4, the only difference among journals that is worth noting is the frequency of confounded replications. $24 \%$ of the replications in AMJ were confounded, whereas $17.4 \%$ of JAP replications and $10.4 \%$ of JOM replications were confounded. Chi-squared comparisons show that the proportion of replications that are confounded is significantly smaller in JOM than in AMJ $(4.47,1 \mathrm{df})$, but that the JAP proportion is not significantly different from either of the other two.

\section{Literal replication studies}

Finally, 19 out of 406 studies (4.7\%) were literal replications, out of which $17(89.5 \%, 17$ out of 19) were dependent literal replications and 2 (10.5\%) were independent literal replications. As such, literal replications are the rarest form of replication studies we observed.

As an example, Martinez, Sawyer, Thoroughgood, Ruggs, and Smith (2017) examined the relationship between authentic identity expression and work-related attitudes and experiences of transgender employees. In studies 1 and 2, the researchers collected data from conference participants at the same conference for transgender health issues in the Northeastern USA. The data collection method and measures were exactly the same in both studies. While the researchers added measurement of mediators in study 2 , the core variables from study 1 that were supposed to be replicated, were replicated literally, i.e., with the exact same study design.

\section{Regressive replication studies}

Finally, we also found an additional category of replication studies that we did not previously conceptualize in our framework, regressive replication studies. While they are not very frequent ( 8 out of 406 studies, $2 \%$ ), it is worth highlighting them. Regressive replication studies are follow-up studies that in fact contain all of the major shortcomings of the studies that 
FRAMEWORK FOR REPLICATION

they were intended to replicate plus at least one additional one. The value of regressive replications is unclear.

In sum, although dependent quasi-random and dependent incremental constructive replications are common, so are forms whose value is unclear (confounded and regressive replications). The forms of replication that target critical design flaws (substantial and comprehensive constructive), especially those that incorporate experimenter issues (independent) are very rare indeed.

\section{DISCUSSION}

The purpose of this paper is to provide a review of current replication types and practices to identify the replication needs of our field. Previous literature has used terms inconsistently to denote different things. In this paper, we identify and provide discussion of various forms of replication and the threats to scientific legitimacy they target.

By distinguishing different forms of replication and by highlighting the specific concerns that each of these forms targets, we provide readers with an evaluation regarding which forms make a superior empirical contribution to science and knowledge creation, and which forms do not (even though they may be more frequently used). Along these lines, we provide concrete examples to showcase substantial and comprehensive constructive replication studies to demonstrate how these studies make empirical contributions that greatly advance knowledge about their phenomena of interest. In doing so, we aim to shift the focus of the research community away from some of the currently popular, but less useful, forms of replication, and towards more constructive and informative replication strategies.

At the outset of the paper, we attempted to reframe the replication crisis. Those who object to claims of a replication crisis point to meta-analyses and multi-study papers as evidence that a replication crisis does not exist. If by "replication crisis" we mean a lack of replications of 
any kind, then these critics are correct. As we show, this is an irrational definition of "replication crisis”. A replication crisis exists not because sampling error has been ignored (it hasn't), but because sources of error such as design flaws and researcher motives have been ignored. Substantive or comprehensive constructive replications would address the former, and independent constructive replications would address the latter. These types of replication are uncommon in the extreme. This is the replication crisis.

Our hope is that the classification of different types of replication would help instructors in their efforts to teach the next generation of organizational scientists about research design. It should also help editors and reviewers in determining the strengths and weaknesses of a particular study. Articles that include independent and highly constructive replication studies are difficult to execute, and researchers may be reluctant to undertake them out of fear of criticism for lack of novelty. Such efforts must be encouraged and nurtured if they are to be undertaken at all.

This last point is of particular importance. In our roles as researchers, authors, reviewers, and editors, we have witnessed a strong imbalance in research and publishing towards theoretical contribution (and more specifically, novelty), resulting in a neglect of the importance of empirical contributions. We are not alone in this. Many have written about the importance of novelty to establish theoretical contribution (e.g., Davis, 1971; Locke and Golden-Biddle, 1997; Sandberg \& Alvesson, 2011; Weick, 1989; Whetten, Rodgers, Green \& Coles, 2014). At the same time, many authors have bemoaned the fact that empirical contributions are being disregarded and viewed as less valuable (e.g., Chan \& Arvey, 2012; Tsang \& Kwan, 1999). We now discuss these issues in more depth, attempting an integration of theoretical and empirical contribution by placing a greater emphasis on the role of replication. 


\section{Theoretical versus empirical contribution and the role of replication}

Replication has a particular place in the scientific process. Popper (2005: 23) wrote:

We do not take even our own observations quite seriously, or accept them as scientific observations, until we have repeated and tested them. Only by such repetitions can we convince ourselves that we are not dealing with a mere isolated 'coincidence', but with events which, on account of their regularity and reproducibility, are in principle intersubjectively testable.

While there is debate about whether social science studies can ever be repeated exactly (e.g., Lykken, 1968; Stroebe \& Strack, 2014), the general idea that a phenomenon should be observable under roughly the same conditions is one to which we believe most researchers would subscribe. Otherwise, the more general goal of prediction becomes unattainable. Yet, as a discipline we seem to afford this component of the research process relatively little attention.

As we explained above, this may in part be due to different understandings of the meaning of replication, and subsequently, its utility. Despite these issues, however, creating theory without ever testing it stringently, or testing it only once and accepting it as gospel is also unhelpful. Our field has seen so much emphasis on theoretical innovation and novelty that theories have sprouted around us like weeds (Leavitt, Mitchell, \& Peterson, 2010) and are threatening to overgrow the few tried-and-tested theories. Moreover, the need for results to support hypotheses has given rise to widespread p-hacking, HARKing, and other such strategies. Nelson, Simmons, and Simonsohn (2018), and Johnson, Payne, Wang, Asher, and Mandal (2017) recently concluded that the prevalence of such strategies has resulted in a false positive rate likely to be even higher than suggested by the OSC. 
Leavitt et al. (2010) suggested that in order to keep our research garden ordered, happy, and healthy we need to engage in systematic theory pruning, which consists of testing and weeding out those theories that don't really hold up under sound methodological scrutiny. Replication is one of our most useful gardening tools here. But in order for it to be most effective, it should be of the constructive kind, and preferably, independent as well. In making constructive research design choices, not only can we test hypotheses in a more stringent manner, we can also more systematically explore the influence of methods factors on the observability of a given effect (e.g., exploring whether the size or direction of an effect is dependent on using a certain type of measure or sample).

Used in this way, replication studies make a strong empirical contribution that provides the foundation for subsequent theoretical contributions. Without strong empirical evidence, we cannot move our theorizing forward, we can only create more new theories. Strong empirical evidence is built, like strong theory, on an improvement and extension of what has been there before. Currently, the lack of importance accorded to empirical contributions means that papers making an empirical contribution, but not a theoretical one, hardly ever get published. With a stronger focus on empirical contribution we could move the field forward by building strategically, meaningfully, and constructively onto existing research and by extending, consolidating, and strengthening our evidence base. In order to do that, we have to understand fully the nature and purpose of replication and include it in our future research designs.

\section{What can we as researchers do?}

We must consider what a good replication study looks like. When designing a constructive replication study, researchers should begin by envisioning an ideal design for addressing the research questions in which they are interested and determine how previous 
designs in that domain stack up against this ideal design. The biggest opportunities for contributions come from domains in which the differences between actual designs and ideal designs are many and profound. It is here that the comprehensive constructive replication is of most use. More generally, researchers should think about the characteristics of previous designs that potentially compromise the validity of the conclusions drawn from the study. These include sampling error, unreliability in measurement, lack of task representativeness, lack of sample representativeness, lack of construct validity of measures, lack of researcher competence, researcher malfeasance, and many more (see Table 1 for a list of threats targeted by different forms of replication studies). Researchers should then think about the improvements that can be included in the next study to ameliorate these threats, without confounding their study by compromising other aspects of the research design.

One additional consideration regarding constructiveness involves the ethical treatment of research participants. As an anonymous reviewer pointed out, there are many notorious examples of studies that were plagued by ethical shortcomings (e.g., the Milgram studies, the Stanford prison experiment, some twin studies). At the same time, these studies taught us some of the most important lessons about human behavior that the behavioral sciences have to offer. One could argue that the biggest flaw in these studies has nothing to do with causal inference or sample representativeness but lies instead in the manner in which participants were treated. One could make a tremendous, top-tier caliber contribution simply by finding a way to uncover truths about obedience and power without subjecting participants to the level of risk created by Milgram, Zimbardo, etc.

Throughout this paper, we highlighted several examples of well-designed constructive replication studies and have described their approach to replication design in depth (e.g., Mathieu 
et al., 2012 or Schultze et al., 2012). Table 1 contains several examples of the various forms of replication that researchers can use as a template for their own replication designs. It is important that the authors of a constructive replication study think carefully about how to improve the most important characteristics of the original study that allows them to better address the research question of interest and draw more valid conclusions. To provide readers with concrete examples for what they can improve in their own research designs, in Table 4 we list the major improvements of the substantial and comprehensive constructive replications we found in our empirical examination. Granted, some studies make more improvements than others. But even one major improvement is a step forward.

Ultimately, we implore researchers to consider a more strategic and systematic approach to the design of follow-up or replication studies to ensure that something is learned about the impact of methodological choices and design characteristics on the effects and relationships that are being studied. In existing research, a great deal of emphasis is placed on substantive moderators, i.e., generalizability, and not enough on the impact of methodological moderators i.e., replicability. This is an important shortcoming that must be addressed in order to better understand how choices related to research design affect our ability to understand workplace phenomena. We as authors need to plan and execute such studies, and we as reviewers and editors must be open to them despite their lack of "theoretical contribution."

Insert Table 4 here

What can we as reviewers and editors do? 
Although the contribution of the independent comprehensive constructive replication is clear, such studies are very rare. This likely is due to several factors. First, authors are not rewarded by journals for submitting papers that do not involve theoretical novelty (Sandberg \& Alvesson, 2011; Locke \& Golden-Biddle, 1997). This is the reason that Edwards, Berry, and Kay (2013) found that models from $A M R$ papers are so seldom tested. Constructive replication predominantly focuses on empirical contribution through improvement rather than on theoretical contribution. Given current publication practices and preferences, researchers may find it difficult to get comprehensive constructive replications through a modern review process that puts so much emphasis on theoretical novelty (Tsang \& Kwan, 1999; Whetten et al., 2014), or what Antonakis (2017) might have called the comorbid diseases of theorrhea and neophilia. Even if such fears were somewhat unfounded, reduced probabilities of success coupled with the difficulty associated with execution of a comprehensive constructive study likely act as a strong deterrent.

In addition, reviewers and editors prefer studies that support previous findings and are more likely to attribute a failure to replicate to some flaw of the follow-up research (e.g., Chan \& Arvey, 2012). To make matters worse, when selecting reviewers of a replication, editors may choose one of the authors of the original study, particularly if the replication suggested different conclusions. This makes the reviewer assignment decision a kind of "performative," as J.L. Austin (1963) would have put it, consigning the replication to the rejection bin before the editor has even read it. As such, a replication that does not find the same results and does not draw the same conclusions as previous work may make the selection of potentially hostile reviewers more likely. We would advocate, though, that it is exactly this type of strong empirical contribution that supports theory through theory pruning (e.g., Leavitt et al., 2010) and theory refinement 
(Edwards, 2010). Consequently, when reviewing replications, especially those with disconfirming outcomes, we encourage editors to be more cautious in their choice of reviewers. Naturally, an editor may want to engage the critical lens of a reviewer that is highly knowledgeable on the topic. In that case, the editor needs to take the potential conflict of interest of the reviewers into account.

Just as important, we encourage reviewers of replications to embrace a sort of "antiperformativity" (Fournier \& Grey, 2000). The term comes from critical management studies (CMS), but it seems to apply just as well to the effort to increase the number of replications in our field. Spicer, Alvesson, and Kärreman (2009), referring to Fournier and Grey (2000), state "By anti-performativity, they mean that CMS should resist attempts to 'develop and celebrate knowledge which contributes to the production of maximum output for minimum input... (p.17)" (Spicer et al., p. 540). By 'input and output' they mean the work and products of employees, and that scholars should resist the drift towards Taylorian managerialism. We can extend the notion of anti-performativity to the present topic by replacing output and input of employees with output and input of management scholars. Reviewers who embrace antiperformativity would engage in active and subversive intervention, to borrow a phrase from Spicer et al. (2009). The intervention would occur in the review process, whereby the reviewer not only refuses to succumb to theorrhea and neophilia, but also anticipates these diseases in others and attempts to inoculate the editor and the other reviewers by making the case that the empirical contribution of the well-executed constructive replication more than makes up for its lack of theoretical contribution. Criticisms such as, "We don't learn anything new from this paper" or "It doesn't contribute to new knowledge" may hold water for confounded or quasirandom or even incremental constructive replications, but they are fatuous in response to 
substantial or comprehensive constructive replication, and the anti-performative reviewer does his or her best to subvert them.

Finally, given that many studies lack comprehensive, transparent description of the methodological choices made by the authors (Aguinis et al., 2018), it is not always easy to design a study that improves upon the most important study characteristics. As a result, the putative replicator might choose a design that they believe to be superior, but which does not actually improve upon the most important design characteristics of the original study. The goal of the replicator might be constructive replication, but the study may nevertheless fall into the category of quasi-random replication. Preregistration might be helpful here because it provides for disclosure of methodological details before recalcitrant results compel the author to omit design details that might cause reviewers to ask uncomfortable questions (Nosek \& Lakens, 2014; Nosek et al., 2018).

To sum up, in order for constructive replication to be probable, we as reviewers and editors need to revisit our implicit weighting schemes. Too often, theoretical novelty acts as a first hurdle in a multiple hurdle system. If a paper doesn't clear this hurdle, then it is out of the race regardless of its other attributes. We also tend to look askance at results that contradict previous findings regardless of the relative virtues of the designs that produced the different results. Even if these obstacles did not exist, constructive replication would be difficult because methodological details are often omitted, frequently at the behest of reviewers and editors who want to shorten manuscripts. Real progress in our field can only come from a rigorous scientific approach, and replication is an essential tool in this approach. Many of us, in our roles as researchers, would like to conduct such studies. In order for this to happen, we in our roles as editors and reviewers must set aside some of the rules that we were taught and by which we have 
lived our professional lives. We must make a conscious effort to replace them with rules that not only allow but actively encourage independent constructive replication. Replication may not be so interesting in the Davis (1971) sense, but it is indispensable. To quote Sir Francis Bacon, "The understanding must not therefore be supplied with wings, but rather hung with weights, to keep it from leaping and flying" (1620/1858, p.97). Novelty is like a hot air balloon, and constructive replication is its ballast. With ballast, the balloon allows us to see the world. Without ballast, the balloon soars away until it has burned its fuel, and then crashes to the ground. So, let's not forget the ballast. 
FRAMEWORK FOR REPLICATION

\section{REFERENCES}

Adams, A.T., Ajrouch, K.J., Henderson, H., \& Heard, I. 2005. Service-learning outcomes research: The role and scarcity of replication studies. Journal of Applied Sociology, 22: 5574.

Aguinis, H., Ramani, R.S., \& Alabduljader, N. 2018. What you see is what you get? Enhancing methodological transparency in management research. Academy of Management Annals, 12: $1-28$.

Aguinis, H., Edwards, J. R., \& Bradley, K. J. 2017. Improving Our Understanding of Moderation and Mediation in Strategic Management Research. Organizational Research Methods, 20: $665-685$.

Anderson, S. F., \& Maxwell, S. E. 2015. There's more than one way to conduct a replication study: Beyond statistical significance. Psychological Methods, 21: 1-12.

Antonakis, J. 2017. Editorial: The future of The Leadership Quarterly. The Leadership Quarterly, 28: 1-4.

Asendorpf, J. B., Conner, M., De Fruyt, F., De Houwer, J., Denissen, J. J. A., Fiedler, K., Fiedler, S., Funder, D.C., Kliegl, R., Nosek, B.A., Perugini, M., Roberts, B.W., Schmitt, M., Vanaken, M.A.G., Weber, H., \& Wicherts, J. M. 2013. Recommendations for increasing replicability in psychology. European Journal of Personality, 27: 108-119.

Austin, J.L. 1962. How To Do Things With Words. Oxford University Press, Oxford.

Bacon, F. 1858. Novum organum. In: J. Spedding, R.L. Ellis, \& D.D. Heath (Eds.), The Works of Francis Bacon: 39-248. London, England: Longman.

Benson, L., \& Borrego, M. 2015. The Role of Replication in Engineering Education Research. Journal of Engineering Education, 104: 388-392. 
Bettis, R. A., Helfat, C. E., \& Shaver, J. M. 2016. The necessity, logic, and forms of replication. Strategic Management Journal, 37: 2193-2203.

Carey, B. 2016. New critique sees flaws in landmark analysis of psychology studies. The New York Times: A3. Retrieved from http://nyti.ms/1OUoPmE.

Carpenter, S. 2012. Psychology’s bold initiative. Science, 335(6076): 1558-1561.

Chan, M. E., \& Arvey, R. D. 2012. Meta-Analysis and the Development of Knowledge. Perspectives on Psychological Science, 7: 79-92.

Chen, M., Chen, C. C., \& Sheldon, O. J. 2016. Relaxing moral reasoning to win: How organizational identification relates to unethical pro-organizational behavior. Journal of Applied Psychology, 7: 1-30.

Conlon, E. J., \& Parks, J. M. 1987. Information requests in the context of escalation. Journal of Applied Psychology, 72: 344-350.

Cortina, J. M. 1993. What is coefficient alpha? An examination of theory and applications. Journal of Applied Psychology, 78: 98-104.

Davis, M. S. 1971. That's interesting! Toward a phenomenology of sociology and a sociology of phenomenology. Philosophy of Social Science, 1: 209-344.

Dillon, R. L., Tinsley, C. H., Madsen, P. M. \& Rogers, E. W. 2016. Organizational correctives for improving recognition of near-miss events. Journal of Management, 42: 671-697.

Easley, R. W., Madden, C. S., \& Dunn, M. G. 2000. Conducting marketing science: The role of replication in the research process. Journal of Business Research, 48: 83-92.

Eden, D., \& Shani, A. B. 1982. Pygmalion goes to boot camp: Expectancy, leadership, and trainee performance. Journal of Applied Psychology, 67: 194-199. 
FRAMEWORK FOR REPLICATION

Edwards, J. R. 2010. Reconsidering theoretical progress in organizational and management research. Organizational Research Methods, 13: 615-619.

Edwards, J. R., Berry, J. W., \& Kay, V. S. 2013. Bridging the great divide between theoretical and empirical management research. Working paper, Kenan-Flagler Business School, University of North Carolina.

Edwards, J. R., \& Harrison, R. V. 1993. Job demands and worker health: Three-dimensional reexamination of the relationship between person-environment fit and strain. Journal of Applied Psychology, 78: 628-648.

Fiedler, K. 2018. The creative cycle and the growth of psychological science. Perspectives on Psychological Science, Vol. 13(4), 433-438.

Fournier, V., \& Grey, C. 2000. At the critical moment: Conditions and prospects for critical management studies. Human Relations, 53: 7-32.

Garcia, P. R. J. M., Restubog, S. L. D., Kiewitz, C., Scott, K. L., \& Tang, R. L. 2014. Roots run deep: Investigating psychological mechanisms between history of family aggression and abusive supervision. Journal of Applied Psychology, 99: 883-897.

Gilbert, D. T., King, G., Pettigrew, S., \& Wilson, T. D. 2016. Comment on: Estimating the reproducibility of psychological science. Science, 351(6277): 1037-a.

Gleser, L. J. 1996. [Bootstrap Confidence Intervals]: Comment. Statistical Science, 11: 219-221.

Gómez, O. S., Juristo, N., \& Vegas, S. 2014. Understanding replication of experiments in software engineering: A classification. Information and Software Technology, 56: 10331048.

Hanek, K. J., Garcia, S. M., \& Tor, A. 2016. Gender and competitive preferences: the role of competition size. Journal of Applied Psychology, 101: 1122-1133. 
Hollenbeck, J. R., DeRue, D. S., \& Mannor, M. 2006. Statistical power and parameter stability when subjects are few and tests are many: Comment on Peterson, Smith, Martorana, and Owens (2003). Journal of Applied Psychology, 91: 1-5.

Johnson, V. E., Payne, R. D., Wang, T., Asher, A., \& Mandal, S. 2017. On the reproducibility of psychological science. Journal of the American Statistical Association, 112: 1-10.

Judge, T. A., Thoresen, C. J., Bono, J. E., \& Patton, G. K. 2001. The job satisfaction-job performance relationship: A qualitative and quantitative review. Psychological Bulletin, 127: $376-407$.

Kelly, C.D. 2006. Replicating empirical research in behavioral ecology: How and why it should be done but rarely ever is. The Quarterly Review of Biology, 81: 221-236.

Kelly, C. W., Chase, L. J., \& Tucker, R. K. 1979. Replication in experimental communication research: An analysis. Human Communication Research, 5: 338-342.

Leavitt, K., Mitchell, R. R., \& Peterson, J. 2010. Theory pruning: Strategies to reduce our dense theoretical landscape. Organizational Research Methods, 13: 644-667.

Locke, K., \& Golden-Biddle, K. 1997. Constructing opportunities for contribution: Structuring intertextual coherence and "problematizing" in organizational studies. Academy of Management Journal, 40: 1023-1062.

Lüdtke, R. 2008. Do that to me one more time! What kind of trial replications do we need? Complementary Therapies in Medicine, 16: 181-182.

Lykken, D. T. 1968. Statistical significance in psychological research. Psychological Bulletin, 70: $151-159$.

Mackey, J. D., Frieder, R. E., Brees, J. R., \& Martinko, M. J. 2017. Abusive supervision: A meta-analysis and empirical review. Journal of Management, 43: 1940-1965. 
FRAMEWORK FOR REPLICATION

Martinez, L. R., Sawyer, K. B., Thoroughgood, C. N., Ruggs, E. N., \& Smith, N. A. 2017. The importance of being "me": The relation between authentic identity expression and transgender employees' work-related attitudes and experiences. Journal of Applied Psychology, 102: 215-226.

Mathieu, J. E., Kukenberger, M. R., D’Innocenzo, L., \& Reilly, G. 2015. Modeling reciprocal team cohesion - performance relationships, as impacted by shared leadership and members' competence. Journal of Applied Psychology, 100: 1-22.

McElreath, R., \& Smaldino, P. E. 2015. Replication, communication, and the population dynamics of scientific discovery. PLoS One, 10: e0136088.

Merton, R. K. 1942/1973. The sociology of science: Theoretical and empirical investigations. University of Chicago Press.

Mittelstaedt, R. A., \& Zorn, T. S. 1984. Econometric replication: Lessons from the experimental sciences. Quarterly Journal of Business and Economics, 23: 9-15.

Nelson, L. D., Simmons, J., \& Simonsohn, U. 2018. Psychology's renaissance. Annual Review of Psychology, 69: 511-534.

Nosek, B. A., \& Lakens, D. 2014. Registered Reports: A method to increase the credibility of published results. Social Psychology, 45: 137-141.

Nosek, B. A., Ebersole, C. R., DeHaven, A. C., \& Mellor, D. T. 2018. The preregistration revolution. Proceedings of the National Academy of Sciences: 201708274.

Open Science Collaboration 2015. Estimating the reproducibility of psychological science. Science, 349: aac4716-1-aac4716-8.

Pashler, H., \& Harris, C. R. 2012. Is the replicability crisis overblown? Three arguments examined. Perspectives on Psychological Science, 7: 531-536. 
Pashler, H., \& Wagenmakers, E.-J. 2012. Editors' introduction to the special section on replicability in Psychological Science: A crisis of confidence? Perspectives on Psychological Science, 7: 528-530.

Popper, K. 2005. The Logic of Scientific Discovery. New York, NY: Taylor \& Francis e-Library. Sandberg, J., \& Alvesson, M. 2011. Ways of constructing research questions: gap-spotting or problematization? Organization, 18: 23-44.

Schultze, T., Pfeiffer, F., \& Schulz-Hardt, S. 2012. Biased information processing in the escalation paradigm: Information search and information evaluation as potential mediators of escalating commitment. Journal of Applied Psychology, 97: 16-32.

Simons, D. J. 2014. The value of direct replication. Perspectives on Psychological Science, 9: $76-80$.

Spicer, A., Alvesson, M., \& Kärreman, D. 2009. Critical performativity: The unfinished business of critical management studies. Human Relations, 62: 537-560.

Stroebe, W., \& Strack, F. 2014. The alleged crisis and the illusion of exact replication. Perspectives on Psychological Science, 9: 59-71.

Thau, S., Derfler-Rozin, R., Pitesa, M., Mitchell, M. S., \& Pillutla, M. M. 2015. Unethical for the Sake of the Group: Risk of Social Exclusion and Pro-Group Unethical Behavior. Journal of Applied Psychology, 100: 98-113.

Tsang, E. W. K., \& Kwan, K.-M. 1999. Replication and theory development in organizational science: A critical realist perspective. Academy of Management Review, 24: 759-780.

Van Iddekinge, C. H., Aguinis, H., Mackey, J. D., \& DeOrtentiis, P. S. 2018. A meta-analysis of the interactive, additive, and relative effects of cognitive ability and motivation on performance. Journal of Management, 44: 249-279. 
FRAMEWORK FOR REPLICATION

Vazire, S. 2018. Implications of the credibility revolution for productivity, creativity, and progress. Perspectives on Psychological Science, 13: 411-417.

Venkataramani, V., Labianca, G., \& Grosser, T. 2013. Positive and negative workplace relationships, social satisfaction, and organizational attachment. Journal of Applied Psychology, 98: 1028-1039.

Wagenmakers, E.J., Dutilh, G., \& Sarafoglou, A. 2018. The creativity-verification cycle in psychological science: new methods to combat old idols. Perspectives on Psychological Science, 13: 418-427.

Weick, K. E. 1989. Theory construction as disciplined imagination. Academy of Management Review, 14: 516-531.

Whetten, D. A., Rodgers, Z. J., Green, C. D., \& Coles, R. S. 2014. What, really, constitutes a theoretical contribution? In Academy of Management Proceedings, 1: 17067.

Whewell, W. 1840. The philosophy of the inductive sciences, founded upon their history (Vol. 2). London, England: John W. Parker. Retrieved from https://archive.org/details/philosophyofindu01whewrich.

* References in tables are available in the online supplementary materials. 


\section{FOOTNOTES}

${ }^{1}$ It should be noted that the term "conceptual replication" is often used merely to denote a replication that is not literal (e.g., Stroebe \& Strack, 2014). A researcher either tries to repeat a previous study in every detail, or s/he conducts a "conceptual replication". In essence, the present paper distinguishes between a conceptual replication that differs from the original study because it employs a superior design (i.e., a constructive study), and a conceptual replication that differs from the original study for reasons other than design superiority (i.e., quasi-random). The latter is very common, the former much less so, and these two different types of "conceptual replication" address very different issues, thus the need for distinct terms.

${ }^{2}$ We conducted our searches both via the search function on the journals' respective websites but also via Ovid, APA, and EBSCO. Different search engines led to different search results (both in numbers and in content). Consequently, we went through all available search results provided from any of the search engines to ensure that we didn't miss any applicable papers.

${ }^{3}$ This type of replication is similar to the type to which Stroebe and Strack (2014) would apply the term conceptual replication, which they define as a test of the relationships among the same "...underlying theoretical variables using different manipulations and/or different measures" (p. 60). However, Stroebe and Strack assume in their discussion of conceptual replication that authors strategically choose other research designs and contexts so that they might learn something about boundary conditions under which an effect can be observed or about factors that influence the relationship between theoretical concepts. As our empirical review of published replication studies shows, however, most authors do not choose their replication design elements for these purposes. There is very seldom discussion of the underlying factors, mechanisms, and structures that may be responsible for the fact that a specific effect size may be observed in one study but not in another.

${ }^{4} N=406$; In our coding, we also found 35 generalizability studies that focused on the testing of a substantial moderator, 64 reproducibility studies, and 3 studies that were neither a replication or reproducibility study. These studies are not included in the table. 


\section{Table 1}

\section{Forms of Replication}

\begin{tabular}{|c|c|c|c|c|}
\hline Form of replication & efinition & Alternative names & Targets concerns about & Examples \\
\hline Literal replication & \multirow{4}{*}{$\begin{array}{l}\text { Repeat exactly the procedures } \\
\text { used in the original study, } \\
\text { including the same sample, same } \\
\text { research design, experimental } \\
\text { setup, measures, procedure, etc. } \\
\text { In its most exact form, literal } \\
\text { replication is not possible. }\end{array}$} & \multirow{4}{*}{$\begin{array}{l}\text { Literal replication (Kelly } \\
\text { et al., 1979; Lykken, } \\
\text { 1968) } \\
\text { Direct replication } \\
\text { (Simons, 2014) } \\
\text { Exact replication } \\
\text { (Stroebe \& Strack, 2014; } \\
\text { Tsang \& Kwan, 1999) }\end{array}$} & & \multirow{4}{*}{$\begin{array}{l}\text { Whiting, } \\
\text { Maynes, } \\
\text { Podsakoff, \& } \\
\text { Podsakoff (2012) }\end{array}$} \\
\hline $\begin{array}{l}\text { Dependent literal } \\
\text { replication }\end{array}$ & & & Sampling error or reporting & \\
\hline & & & & \\
\hline $\begin{array}{l}\text { Independent literal } \\
\text { replication }\end{array}$ & & & $\begin{array}{l}\text { Sampling error or reporting } \\
\text { accuracy, experimenter } \\
\text { competence regarding research } \\
\text { design, experimenter } \\
\text { malfeasance }\end{array}$ & \\
\hline Quasi-random replication & \multirow{3}{*}{$\begin{array}{l}\text { Repeat some of the procedures } \\
\text { and design characteristics used } \\
\text { in the original study, but with } \\
\text { variation in some of the design } \\
\text { characteristics in ways that are } \\
\text { not driven by a desire to } \\
\text { improve upon the previous study } \\
\text { (e.g., convenience, familiarity } \\
\text { with the research method or } \\
\text { setting, desire for supportive } \\
\text { results). }\end{array}$} & \multirow{3}{*}{$\begin{array}{l}\text { Conceptual replication } \\
\text { (Stroebe \& Strack, 2014) } \\
\text { Instrumental replication } \\
\text { (Kelly et al., 1979) } \\
\text { Operational and } \\
\text { instrumental replication } \\
\text { (Kelly et al., 1979) } \\
\text { Replicability (Asendorpf } \\
\text { et al., 2013) } \\
\text { Conceptual extension } \\
\text { (Tsang \& Kwan, 1999) }\end{array}$} & & \multirow{3}{*}{$\begin{array}{l}\text { Dependent: } \\
\text { Venkataramani, } \\
\text { Labianca, \& } \\
\text { Grosser, (2013) } \\
\text { Independent: } \\
\text { Van Iddekinge et } \\
\text { al. (2018) }\end{array}$} \\
\hline $\begin{array}{l}\text { Dependent quasi- } \\
\text { random replication }\end{array}$ & & & $\begin{array}{l}\text { Sampling error, unanticipated } \\
\text { methodological moderators }\end{array}$ & \\
\hline $\begin{array}{l}\text { Independent quasi- } \\
\text { random replication }\end{array}$ & & & $\begin{array}{l}\text { Sampling error, unanticipated } \\
\text { methodological moderators, } \\
\text { experimental competence, } \\
\text { experiment malfeasance, } \\
\text { reporting accuracy }\end{array}$ & \\
\hline Constructive replication & \multirow{2}{*}{$\begin{array}{l}\text { Improve upon previous efforts } \\
\text { by employing more valid } \\
\text { measures, more critical control } \\
\text { variables, a more realistic task, a } \\
\text { more representative sample, or a } \\
\text { design that allows for stronger } \\
\text { conclusions regarding causality } \\
\text { while retaining the virtues of }\end{array}$} & \multirow[t]{2}{*}{$\begin{array}{l}\text { Conceptual replication } \\
\text { (Stroebe \& Strack, 2014) }\end{array}$} & $\begin{array}{l}\text { Flaws in previous assessments } \\
\text { of propositions, unfounded } \\
\text { assumptions with regard to } \\
\text { design characteristics. }\end{array}$ & \multirow{2}{*}{$\begin{array}{l}\text { Dependent: } \\
\text { Mathieu et al. } \\
\text { (2015, sub.) } \\
\text { Loschelder et al. } \\
\text { (2016, incr.) } \\
\text { Wu et al. (2016, } \\
\text { incr.) }\end{array}$} \\
\hline $\begin{array}{l}\text { Dependent } \\
\text { constructive } \\
\text { replication }\end{array}$ & & & $\begin{array}{l}\text { Methodological flaws from } \\
\text { prior tests of the relationship }\end{array}$ & \\
\hline
\end{tabular}




\begin{tabular}{|c|c|c|c|}
\hline $\begin{array}{l}\text { (incl. incremental, } \\
\text { substantial, } \\
\text { comprehensive) }\end{array}$ & \multirow{2}{*}{$\begin{array}{l}\text { previous efforts. } \\
\text { Repeat some components of the } \\
\text { original test (thereby retaining } \\
\text { its virtues) but improve upon the } \\
\text { original in one or more } \\
\text { important ways. }\end{array}$} & & $\begin{array}{l}\text { Hochwarter et al. } \\
(2007 \text {, sub.) } \\
\text { Garcia et al. }\end{array}$ \\
\hline $\begin{array}{l}\text { Independent } \\
\text { constructive } \\
\text { replication } \\
\text { (incl. incremental, } \\
\text { substantial, } \\
\text { comprehensive) }\end{array}$ & & $\begin{array}{l}\text { Methodological flaws from } \\
\text { prior tests, experimenter } \\
\text { competence, experimenter } \\
\text { malfeasance, reporting } \\
\text { accuracy. }\end{array}$ & $\begin{array}{l}\text { (2014, comp.) } \\
\text { Independent: } \\
\text { Eden and Shani } \\
\text { (1982, comp.) } \\
\text { Schultze et al. } \\
\text { (2012, comp.) }\end{array}$ \\
\hline Confounded replication & \multirow{3}{*}{$\begin{array}{l}\text { Improve upon one or more } \\
\text { aspects of a study (or at least } \\
\text { investigate the possibility of a } \\
\text { flaw in the original) while } \\
\text { compromising another aspect. }\end{array}$} & \multirow[t]{3}{*}{ None } & Dependent: \\
\hline Dependent & & & Chen et al. \\
\hline Independent & & & $\begin{array}{l}\text { Long \& Christian } \\
\text { (2015) } \\
\text { Independent: } \\
\text { Wu \& Griffin } \\
(2012)\end{array}$ \\
\hline Regressive replication & \multirow{3}{*}{$\begin{array}{l}\text { Has more shortcomings than the } \\
\text { research design of the original } \\
\text { study and often worsens the } \\
\text { methodological rigor of the } \\
\text { hypothesis test in the previous } \\
\text { study. }\end{array}$} & \multirow[t]{3}{*}{ None } & \multirow{3}{*}{$\begin{array}{l}\text { Shoss, } \\
\text { Eisenberger, } \\
\text { Restubog, \& } \\
\text { Zagenczyk } \\
\text { (2013, study 2) } \\
\text { Strauss, Griffin, } \\
\text { \& Parker (2012, } \\
\text { study 1b) } \\
\text { Harold, Oh, } \\
\text { Holtz, Han, \& } \\
\text { Giacolone (2016, } \\
\text { study 2) }\end{array}$} \\
\hline Dependent & & & \\
\hline Independent & & & \\
\hline
\end{tabular}


FRAMEWORK FOR REPLICATION

2

3

4

5

6

7

8

9

10

11

12

\section{Table 2}

Frequency of type of replication study employed in papers published across JAP, AMJ, and $\mathrm{JOM}^{4}$

\begin{tabular}{|l|c|c|c|}
\hline & \multicolumn{3}{|c|}{ Replication } \\
\hline Type & Dependent & Independent & Semi-independent \\
\hline Literal & $17(4.2 \%)$ & $2(0.5 \%)$ & $8(2 \%)$ \\
\hline Quasi-random & $135(33.3 \%)$ & $68(16.7 \%)$ & \\
\hline Constructive & & & \\
\hline Incremental & $64(15.8 \%)$ & $7(1.7 \%)$ & \\
\hline Substantial & $25(6.2 \%)$ & & \\
\hline Comprehensive & $1(0.2 \%)$ & & \\
\hline Confounded & $69(17 \%)$ & $2(0.5 \%)$ & \\
\hline Regressive & $8(2 \%)$ & & \\
\hline Total & $319(78.6 \%)$ & $79(19.5 \%)$ & \\
\hline
\end{tabular}


FRAMEWORK FOR REPLICATION

Table 3

Frequency of type of replication study employed in papers published in JAP, AMJ, and $\mathrm{JOM}^{4}$

\begin{tabular}{|l|c|c|c|}
\hline Type & AMJ & JAP & JOM \\
\hline Constructive & & & 4 \\
\hline Dep. Incremental & 8 & 17 & 3 \\
\hline Dep. Substantial & 5 & 6 & \\
\hline Dep. Comprehensive & & & \\
\hline Ind. Incremental & 1 & & \\
\hline Ind. Substantial & & & \\
\hline Ind. Comprehensive & & & \\
\hline & & & \\
\hline Semi-ind. Incremental & & & \\
\hline & & & \\
\hline Literal & & & \\
\hline Dependent & 2 & & \\
\hline Independent & & & \\
\hline & & & \\
\hline Quasi-random & & & \\
\hline Dependent & & & \\
\hline Independent & & & \\
\hline Semi-independent & & & \\
\hline & & & \\
\hline Confounded & & & \\
\hline Dependent & & & \\
\hline Independent & & & \\
\hline Regressive & & & \\
\hline Dependent & & & \\
\hline Independent & & & \\
\hline Total & & & \\
\hline
\end{tabular}


FRAMEWORK FOR REPLICATION

Table 4

Methodological improvements in substantial and comprehensive constructive replications

\begin{tabular}{|c|c|c|c|}
\hline $\begin{array}{l}\text { Jour } \\
\text { nal }\end{array}$ & Study & $\begin{array}{l}\text { Which } \\
\text { repetition }\end{array}$ & Improvements over original study \\
\hline \multicolumn{4}{|c|}{ Dependent substantial constructive replication } \\
\hline AMJ & $\begin{array}{l}\text { Carson Marr \& Cable } \\
\text { (2014) }\end{array}$ & $\begin{array}{l}\text { study } 2 \text { of } \\
\text { study } 1\end{array}$ & $\begin{array}{l}\text { Field sample, predictions of applicants' future success } \\
9 \text { months and } 2 \text { years after they were accepted into the } \\
\text { program }\end{array}$ \\
\hline AMJ & $\begin{array}{l}\text { Dineen, Duffy, Henle, \& } \\
\text { Kiyoung (2017) }\end{array}$ & $\begin{array}{l}\text { study } 2 \text { of } \\
\text { study } 1\end{array}$ & $\begin{array}{l}\text { More representative sample, two contexts, high } \\
\text { fidelity, additional control variable, longitudinal. }\end{array}$ \\
\hline AMJ & $\begin{array}{l}\text { Jacquart \& Antonakis } \\
\text { (2015) }\end{array}$ & $\begin{array}{l}\text { study } 2 \text { of } \\
\text { study } 1\end{array}$ & $\begin{array}{l}\text { Study 1: archival data, economic modeling; Study 2: } \\
\text { created a TV business report with desired properties, } \\
\text { manipulated leader charisma and company } \\
\text { performance; more experimental control. }\end{array}$ \\
\hline AMJ & $\begin{array}{l}\text { Lopez-Kidwell, Grosser, } \\
\text { Dineen, \& Borgatti } \\
\text { (2013) }\end{array}$ & $\begin{array}{l}\text { study } 2 \text { of } \\
\text { study } 1\end{array}$ & $\begin{array}{l}\text { More representative sample, more specific context } \\
\text { measurement, better identification of peer evaluator }\end{array}$ \\
\hline AMJ & $\begin{array}{l}\text { Wo, Ambrose, \& } \\
\text { Schminke (2015) }\end{array}$ & study 2 of 1 & $\begin{array}{l}\text { Measures of IVs, mediators, and DVs are separated in } \\
\text { time, more specific measures for some of the } \\
\text { variables, larger sample. }\end{array}$ \\
\hline JAP & Adam \& Shirako (2013) & $\begin{array}{l}\text { study } 2 \text { of } \\
\text { study } 1\end{array}$ & $\begin{array}{l}\text { Subtler manipulation of culture. More control through } \\
\text { using the same scenario for everybody. Involved an } \\
\text { actual negotiation offer. }\end{array}$ \\
\hline JAP & $\begin{array}{l}\text { Bennett, Gabriel, } \\
\text { Calderwood, Dahling, \& } \\
\text { Trougakos (2016) }\end{array}$ & $\begin{array}{l}\text { Study } 2 \text { of } \\
\text { study } 1\end{array}$ & $\begin{array}{l}\text { More representative sample, measures separated in } \\
\text { time, some variables measured by a different source, } \\
\text { higher fidelity context. }\end{array}$ \\
\hline JAP & $\begin{array}{l}\text { Bonner, Greenbaum, \& } \\
\text { Quade (2017) }\end{array}$ & $\begin{array}{l}\text { study } 3 \text { of } \\
\text { studies } 1 \text { and } \\
2\end{array}$ & $\begin{array}{l}\text { More representative sample, supervisor ratings of } \\
\text { subordinate unethical behavior }\end{array}$ \\
\hline JAP & $\begin{array}{l}\text { Greenbaum, Quade, } \\
\text { Mawritz, Kim, \& } \\
\text { Crosby (2014) }\end{array}$ & $\begin{array}{l}\text { study } 2 \text { of } \\
\text { study } 1\end{array}$ & $\begin{array}{l}\text { Created a measure of core variable with better } \\
\text { psychometric properties (and include validation } \\
\text { evidence in the Appendix), included additional control } \\
\text { variable, time-lagged surveys }\end{array}$ \\
\hline JAP & $\begin{array}{l}\text { Gunia, Brett, } \\
\text { Nandkeolyar, \& Kamdar } \\
\text { (2011) }\end{array}$ & $\begin{array}{l}\text { study } 3 \text { of } \\
\text { study } 1\end{array}$ & $\begin{array}{l}\text { Audio record negotiation and then have trained coders } \\
\text { code it to have a more objective measure of behaviors, } \\
\text { causal order is now reflected in measurement. }\end{array}$ \\
\hline JAP & $\begin{array}{l}\text { Harman, Ellington, } \\
\text { Surface, \& Thompson } \\
(2015)\end{array}$ & $\begin{array}{l}\text { study } 3 \text { of } \\
\text { studies } 1 \text { and } \\
2\end{array}$ & $\begin{array}{l}\text { Created another high-fidelity setting, behaviorally } \\
\text { focused measure of instructor behavior rather than } \\
\text { perceptive. }\end{array}$ \\
\hline JAP & $\begin{array}{l}\text { Hochwarter, Ferris, } \\
\text { Zinko, Arnell, \& James } \\
\text { (2007) }\end{array}$ & Study 2 of 1 & $\begin{array}{l}\text { More representative sample; supervisor rather than } \\
\text { self-rated performance. }\end{array}$ \\
\hline
\end{tabular}




\begin{tabular}{|c|c|c|c|}
\hline $\begin{array}{l}\text { Jour } \\
\text { nal }\end{array}$ & Study & $\begin{array}{l}\text { Which } \\
\text { repetition }\end{array}$ & Improvements over original study \\
\hline JAP & Jiang \& Probst (2017) & $\begin{array}{l}\text { study } 2 \text { of } \\
\text { study } 1\end{array}$ & $\begin{array}{l}\text { Multi-item measures and more comprehensive burnout } \\
\text { measure. }\end{array}$ \\
\hline JAP & Kinias \& Sim (2016) & $\begin{array}{l}\text { Study } 2 \text { of } \\
\text { Study } 1\end{array}$ & $\begin{array}{l}\text { Used random assignment to conditions, added a } \\
\text { control condition }\end{array}$ \\
\hline JAP & $\begin{array}{l}\text { Krumm, Lievens, } \\
\text { Huffmeier, Lipnevich, } \\
\text { Bendels, \& Hertel } \\
(2015)\end{array}$ & $\begin{array}{l}\text { Study } 1, \\
\text { student vs. } \\
\text { working } \\
\text { samples }\end{array}$ & More representative sample \\
\hline JAP & $\begin{array}{l}\text { Mathieu, Kukenberger, } \\
\text { D'Innocenzo, \& Reilly } \\
(2015)\end{array}$ & study 2 of 1 & $\begin{array}{l}\text { Larger sample, and assessed shared leadership over } \\
\text { time so that it could be studied as emergent }\end{array}$ \\
\hline JAP & $\begin{array}{l}\text { Owens, Baker, Sumpter, } \\
\text { \& Cameron (2016) }\end{array}$ & Study 4 of 3 & $\begin{array}{l}\text { Objective performance measure, additional controls } \\
\text { relative to Study } 3\end{array}$ \\
\hline JAP & $\begin{array}{l}\text { Sinha, Janardhanan, } \\
\text { Greer, Conlon, \& } \\
\text { Edwards (2016) }\end{array}$ & $\begin{array}{l}\text { study } 2 \text { of } \\
\text { study } 1\end{array}$ & $\begin{array}{l}\text { Real work groups for higher fidelity context, } \\
\text { organizational setting in multinational finance } \\
\text { corporation }\end{array}$ \\
\hline JAP & $\begin{array}{l}\text { Sliter, Sinclair, Yuan, \& } \\
\text { Mohr (2014) }\end{array}$ & Study 2 of 1 & $\begin{array}{l}\text { More representative sample, better psychometric } \\
\text { properties of measures of core variables, objective } \\
\text { measure of DV }\end{array}$ \\
\hline JAP & $\begin{array}{l}\text { Van Kleef, van den } \\
\text { Berg, \& Heerdink } \\
(2015)\end{array}$ & experiment 4 & $\begin{array}{l}\text { More realistic question, more relevant emotions, } \\
\text { audio-visual manipulation }\end{array}$ \\
\hline JAP & $\begin{array}{l}\text { Wang \& Murnighan } \\
\text { (2017) }\end{array}$ & $\begin{array}{l}\text { study } 3 \text { of } 1 \\
\text { and } 2\end{array}$ & Behavioral rather than self-reported DV \\
\hline JAP & $\begin{array}{l}\text { Zhu, Aquino, \& Vadera } \\
\text { (2016) }\end{array}$ & $\begin{array}{l}\text { study } 3 \text { of } \\
\text { study } 1\end{array}$ & $\begin{array}{l}\text { Interpretations of a real, current event, lagged rather } \\
\text { than cross-sectional design }\end{array}$ \\
\hline JOM & $\begin{array}{l}\text { Kukenberger, Mathieu, } \\
\text { \& Ruddy (2015) }\end{array}$ & $\begin{array}{l}\text { study } 2 \text { of } \\
\text { study } 1\end{array}$ & $\begin{array}{l}\text { Introduced time lag between group and individual } \\
\text { measures and now use an intervention. }\end{array}$ \\
\hline JOM & $\begin{array}{l}\text { Steel, Kammeyer- } \\
\text { Mueller, \& Paterson } \\
\text { (2015) }\end{array}$ & $\begin{array}{l}\text { previous } \\
\text { research }\end{array}$ & $\begin{array}{l}\text { Increased the number of simulations, used an informed } \\
\text { prior, employed several techniques to prevent bias, } \\
\text { used superior variance estimation technique, also } \\
\text { included the average of individual correlations in their } \\
\text { simulation }\end{array}$ \\
\hline JOM & Vogel \& Mitchell (2017) & $\begin{array}{l}\text { study } 2 \text { of } \\
\text { study } 1\end{array}$ & $\begin{array}{l}\text { More representative sample, i.e., full-time employees } \\
\text { across a range of organizations and industries }\end{array}$ \\
\hline \multicolumn{4}{|c|}{ Dependent comprehensive constructive replication } \\
\hline JAP & $\begin{array}{l}\text { Garcia, Restubog, } \\
\text { Kiewitz, Scott, \& Tang } \\
(2014)\end{array}$ & $\begin{array}{l}\text { Study } 3 \text { of } 1 \\
\text { and } 2\end{array}$ & $\begin{array}{l}\text { More valid measure of family history of aggression } \\
\text { and has a temporal element. }\end{array}$ \\
\hline
\end{tabular}




\section{Figure 1}

Figure 1a. Illustration of dependent versus independent reproducibility study

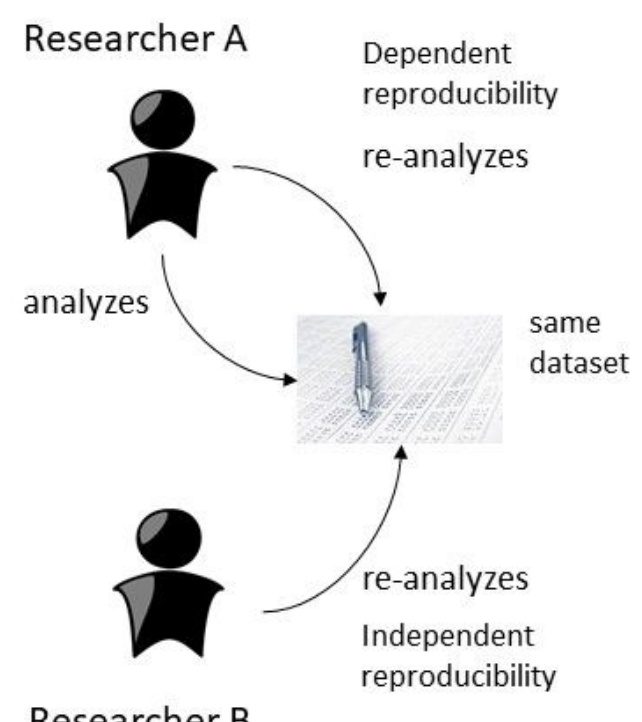

Figure 1b. Illustration of dependent versus independent replication study

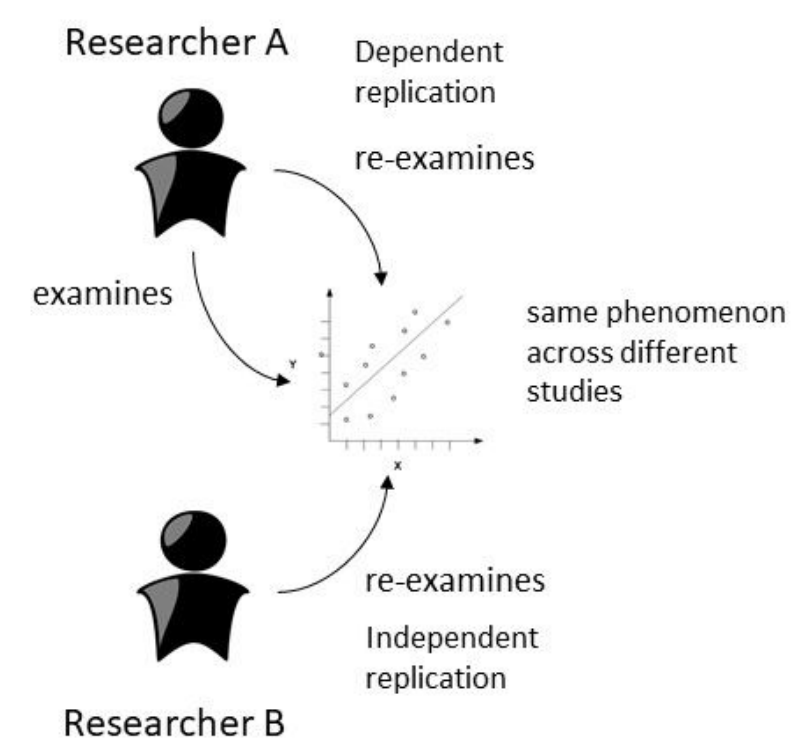


55

56

57

58

59 
Supplementary Materials A: Coding Sheet

\begin{tabular}{|c|c|c|c|c|c|c|c|}
\hline$\#$ & Journal & Search & Study & $\begin{array}{l}\text { Which } \\
\text { repetition }\end{array}$ & Terminology & Independence & $\begin{array}{l}\text { Type of replication or } \\
\text { reproducibility study }\end{array}$ \\
\hline 1 & AMJ & full text & $\begin{array}{l}\text { Ballesteros, Useem, } \\
\& \text { Wry (2017) }\end{array}$ & study 1 & $\begin{array}{l}\text { replication or } \\
\text { replicate }\end{array}$ & dependent & $\begin{array}{l}\text { dependent quasi-random } \\
\text { reproducibility }\end{array}$ \\
\hline 2 & AMJ & $\begin{array}{l}\text { abstract } \\
\& \text { title }\end{array}$ & $\begin{array}{l}\text { Barrick, Bradley, } \\
\text { Kristof-Brown, \& } \\
\text { Colbert (2007) }\end{array}$ & $\begin{array}{l}\text { study } 1 \text { of } \\
\text { previous } \\
\text { research }\end{array}$ & $\begin{array}{l}\text { constructive } \\
\text { replication }\end{array}$ & independent & $\begin{array}{l}\text { independent incremental } \\
\text { constructive replication }\end{array}$ \\
\hline 3 & AMJ & full text & $\begin{array}{l}\text { Bendersky \& } \\
\text { Parikh Shah (2013) }\end{array}$ & study 2 of study & $\begin{array}{l}\text { replication or } \\
\text { replicate }\end{array}$ & dependent & $\begin{array}{l}\text { dependent confounded } \\
\text { replication }\end{array}$ \\
\hline 4 & AMJ & full text & $\begin{array}{l}\text { Boivie, Graffin, \& } \\
\text { Pollock (2012) }\end{array}$ & $\begin{array}{l}\text { study } 1 \text { of } \\
\text { previous } \\
\text { research }\end{array}$ & $\begin{array}{l}\text { replication or } \\
\text { replicate }\end{array}$ & independent & $\begin{array}{l}\text { independent quasi-random } \\
\text { replication }\end{array}$ \\
\hline 5 & AMJ & full text & $\begin{array}{l}\text { Briscoe \& Joshi } \\
(2017)\end{array}$ & study 1 & $\begin{array}{l}\text { replication or } \\
\text { replicate }\end{array}$ & dependent & $\begin{array}{l}\text { dependent quasi-random } \\
\text { reproducibility }\end{array}$ \\
\hline 6 & AMJ & full text & $\begin{array}{l}\text { Briscoe, Chin, \& } \\
\text { Hambrick (2014) }\end{array}$ & study 1 & $\begin{array}{l}\text { replication or } \\
\text { replicate }\end{array}$ & dependent & $\begin{array}{l}\text { dependent quasi-random } \\
\text { reproducibility }\end{array}$ \\
\hline 7 & $\mathrm{AMJ}$ & full text & $\begin{array}{l}\text { Carson Marr \& } \\
\text { Cable (2014) }\end{array}$ & $\begin{array}{l}\text { sample B of } \\
\text { sample A in } \\
\text { study } 2\end{array}$ & $\begin{array}{l}\text { constructive } \\
\text { replication }\end{array}$ & dependent & $\begin{array}{l}\text { dependent quasi-random } \\
\text { replication }\end{array}$ \\
\hline 8 & AMJ & full text & $\begin{array}{l}\text { Carson Marr \& } \\
\text { Cable (2014) }\end{array}$ & $\begin{array}{l}\text { study } 2 \text { of study } \\
1\end{array}$ & Nothing & dependent & $\begin{array}{l}\text { dependent substantial } \\
\text { constructive replication }\end{array}$ \\
\hline
\end{tabular}




\begin{tabular}{|c|c|c|c|c|c|c|c|}
\hline \# & Journal & Search & Study & $\begin{array}{l}\text { Which } \\
\text { repetition }\end{array}$ & Terminology & Independence & $\begin{array}{l}\text { Type of replication or } \\
\text { reproducibility study }\end{array}$ \\
\hline 9 & AMJ & full text & $\begin{array}{l}\text { Carson Marr \& } \\
\text { Thau (2014) }\end{array}$ & $\begin{array}{l}\text { study } 2 \text { of study } \\
1\end{array}$ & $\begin{array}{l}\text { constructive } \\
\text { replication }\end{array}$ & dependent & $\begin{array}{l}\text { dependent confounded } \\
\text { replication }\end{array}$ \\
\hline 10 & AMJ & full text & $\begin{array}{l}\text { Carson Marr \& } \\
\text { Thau (2014) }\end{array}$ & $\begin{array}{l}\text { study } 3 \text { of study } \\
2 \text { and } 1\end{array}$ & Nothing & dependent & $\begin{array}{l}\text { dependent quasi-random } \\
\text { replication }\end{array}$ \\
\hline 11 & AMJ & full text & $\begin{array}{l}\text { Carton, Murphy, \& } \\
\text { Clark (2014) }\end{array}$ & $\begin{array}{l}\text { study } 2 \text { of study } \\
1\end{array}$ & $\begin{array}{l}\text { constructive } \\
\text { replication }\end{array}$ & dependent & $\begin{array}{l}\text { dependent confounded } \\
\text { replication }\end{array}$ \\
\hline 12 & AMJ & $\begin{array}{l}\text { abstract } \\
\& \text { title }\end{array}$ & $\begin{array}{l}\text { Černe, Nerstad, } \\
\text { Dysvik, \& } \\
\text { Škerlavaj (2014) }\end{array}$ & $\begin{array}{l}\text { study } 2 \text { of study } \\
1\end{array}$ & $\begin{array}{l}\text { replication or } \\
\text { replicate }\end{array}$ & dependent & $\begin{array}{l}\text { dependent confounded } \\
\text { replication }\end{array}$ \\
\hline 13 & AMJ & full text & $\begin{array}{l}\text { Chen, Ferris, Kwan, } \\
\text { Yan, Zhou, \& Hong } \\
\text { (2013) }\end{array}$ & $\begin{array}{l}\text { study } 2 \text { of study } \\
1\end{array}$ & $\begin{array}{l}\text { replication or } \\
\text { replicate }\end{array}$ & dependent & $\begin{array}{l}\text { dependent quasi-random } \\
\text { replication }\end{array}$ \\
\hline 14 & AMJ & $\begin{array}{l}\text { abstract } \\
\& \text { title }\end{array}$ & $\begin{array}{l}\text { Courtright, } \\
\text { Gardner, Smith, } \\
\text { McCormick, \& } \\
\text { Colbert (2016) }\end{array}$ & $\begin{array}{l}\text { study } 2 \text { of study } \\
1\end{array}$ & $\begin{array}{l}\text { replicate and } \\
\text { extend }\end{array}$ & dependent & $\begin{array}{l}\text { dependent regressive } \\
\text { replication }\end{array}$ \\
\hline 15 & AMJ & full text & $\begin{array}{l}\text { Crilly, Hansen, \& } \\
\text { Zollo (2016) }\end{array}$ & study 1 & $\begin{array}{l}\text { replication or } \\
\text { replicate }\end{array}$ & dependent & $\begin{array}{l}\text { dependent constructive } \\
\text { reproducibility }\end{array}$ \\
\hline 16 & AMJ & full text & $\begin{array}{l}\text { De Vries, Walter, } \\
\text { Van Der Vegt, \& } \\
\text { Essens (2014) }\end{array}$ & $\begin{array}{l}\text { study } 2 \text { of study } \\
1\end{array}$ & $\begin{array}{l}\text { constructive } \\
\text { replication }\end{array}$ & dependent & $\begin{array}{l}\text { dependent incremental } \\
\text { constructive replication }\end{array}$ \\
\hline
\end{tabular}




\begin{tabular}{|c|c|c|c|c|c|c|c|}
\hline \# & Journal & Search & Study & $\begin{array}{l}\text { Which } \\
\text { repetition }\end{array}$ & Terminology & Independence & $\begin{array}{l}\text { Type of replication or } \\
\text { reproducibility study }\end{array}$ \\
\hline 17 & AMJ & full text & Desai (2015) & study 1 & $\begin{array}{l}\text { replication or } \\
\text { replicate }\end{array}$ & dependent & $\begin{array}{l}\text { dependent quasi-random } \\
\text { reproducibility }\end{array}$ \\
\hline 18 & AMJ & full text & Desai (2016) & study 1 & $\begin{array}{l}\text { replication or } \\
\text { replicate }\end{array}$ & dependent & $\begin{array}{l}\text { dependent quasi-random } \\
\text { reproducibility }\end{array}$ \\
\hline 19 & AMJ & $\begin{array}{l}\text { abstract } \\
\& \text { title }\end{array}$ & $\begin{array}{l}\text { Dineen, Duffy, } \\
\text { Henle, \& Kiyoung } \\
\text { (2017) }\end{array}$ & $\begin{array}{l}\text { study } 2 \text { of study } \\
1\end{array}$ & $\begin{array}{l}\text { constructive } \\
\text { replication }\end{array}$ & dependent & $\begin{array}{l}\text { dependent substantial } \\
\text { constructive replication }\end{array}$ \\
\hline 20 & AMJ & $\begin{array}{l}\text { abstract } \\
\& \text { title }\end{array}$ & $\begin{array}{l}\text { Dineen, Noe, Shaw, } \\
\text { Duffy, \& Wiethoff } \\
(2007) .\end{array}$ & $\begin{array}{l}\text { study } 2 \text { of study } \\
1\end{array}$ & $\begin{array}{l}\text { replication or } \\
\text { replicate }\end{array}$ & dependent & $\begin{array}{l}\text { dependent quasi-random } \\
\text { replication }\end{array}$ \\
\hline 21 & AMJ & $\begin{array}{l}\text { abstract } \\
\& \text { title }\end{array}$ & $\begin{array}{l}\text { Duffy, Scott, Shaw, } \\
\text { Tepper, \& Aquino } \\
(2012)\end{array}$ & $\begin{array}{l}\text { study } 2 \text { of study } \\
1\end{array}$ & $\begin{array}{l}\text { constructive } \\
\text { replication }\end{array}$ & dependent & generalizability \\
\hline 22 & $\mathrm{AMJ}$ & $\begin{array}{l}\text { abstract } \\
\& \text { title }\end{array}$ & $\begin{array}{l}\text { Erez, Schilpzand, } \\
\text { Leavitt, Woolum, } \\
\text { \& Judge (2015) }\end{array}$ & $\begin{array}{l}\text { study } 2 \text { of study } \\
1\end{array}$ & $\begin{array}{l}\text { replication or } \\
\text { replicate }\end{array}$ & dependent & $\begin{array}{l}\text { dependent confounded } \\
\text { replication }\end{array}$ \\
\hline 23 & AMJ & $\begin{array}{l}\text { abstract } \\
\& \text { title }\end{array}$ & $\begin{array}{l}\text { Farh, Lanaj, \& Ilies } \\
\text { (2017) }\end{array}$ & $\begin{array}{l}\text { study } 2 \text { of study } \\
1\end{array}$ & $\begin{array}{l}\text { constructive } \\
\text { replication }\end{array}$ & dependent & $\begin{array}{l}\text { dependent confounded } \\
\text { replication }\end{array}$ \\
\hline 24 & AMJ & full text & $\begin{array}{l}\text { Farooq, Rupp, \& } \\
\text { Farooq (2017) }\end{array}$ & $\begin{array}{l}\text { study } 1 \text { of } \\
\text { previous } \\
\text { research }\end{array}$ & $\begin{array}{l}\text { replicate and } \\
\text { extend }\end{array}$ & independent & $\begin{array}{l}\text { independent quasi-random } \\
\text { replication }\end{array}$ \\
\hline
\end{tabular}




\begin{tabular}{|c|c|c|c|c|c|c|c|}
\hline \# & Journal & Search & Study & $\begin{array}{l}\text { Which } \\
\text { repetition }\end{array}$ & Terminology & Independence & $\begin{array}{l}\text { Type of replication or } \\
\text { reproducibility study }\end{array}$ \\
\hline 25 & AMJ & full text & $\begin{array}{l}\text { Farooq, Rupp, \& } \\
\text { Farooq (2017) }\end{array}$ & $\begin{array}{l}\text { study } 2 \text { of study } \\
1\end{array}$ & $\begin{array}{l}\text { replication or } \\
\text { replicate }\end{array}$ & dependent & $\begin{array}{l}\text { dependent quasi-random } \\
\text { replication }\end{array}$ \\
\hline 26 & AMJ & full text & $\begin{array}{l}\text { Ferris, Lian, } \\
\text { Brown, \& Morrison } \\
(2015)\end{array}$ & $\begin{array}{l}\text { Sample B of } \\
\text { Sample A }\end{array}$ & $\begin{array}{l}\text { replication or } \\
\text { replicate }\end{array}$ & dependent & $\begin{array}{l}\text { dependent incremental } \\
\text { constructive replication }\end{array}$ \\
\hline 27 & AMJ & full text & $\begin{array}{l}\text { Ferris, Ming, Lim, } \\
\text { Yuanyi, \& Fatimah } \\
(2016)\end{array}$ & $\begin{array}{l}\text { study } 2 \text { of study } \\
1\end{array}$ & $\begin{array}{l}\text { constructive } \\
\text { replication }\end{array}$ & dependent & $\begin{array}{l}\text { dependent incremental } \\
\text { constructive replication }\end{array}$ \\
\hline 28 & AMJ & full text & $\begin{array}{l}\text { Firth, Chen, } \\
\text { Kirkman, \& Kim } \\
(2014)\end{array}$ & study 1 & $\begin{array}{l}\text { replication or } \\
\text { replicate }\end{array}$ & dependent & $\begin{array}{l}\text { dependent quasi-random } \\
\text { reproducibility }\end{array}$ \\
\hline 29 & AMJ & full text & Flammer (2013) & study 1 & $\begin{array}{l}\text { replication or } \\
\text { replicate }\end{array}$ & dependent & $\begin{array}{l}\text { dependent literal } \\
\text { reproducibility }\end{array}$ \\
\hline 30 & $\mathrm{AMJ}$ & full text & $\begin{array}{l}\text { Gabriel \& } \\
\text { Diefendorff (2015) }\end{array}$ & $\begin{array}{l}\text { study } 1 \text { of } \\
\text { previous } \\
\text { research }\end{array}$ & $\begin{array}{l}\text { constructive } \\
\text { replication }\end{array}$ & independent & generalizability \\
\hline 31 & AMJ & full text & $\begin{array}{l}\text { Galunic, Ertug, \& } \\
\text { Gargiulo (2012) }\end{array}$ & $\begin{array}{l}\text { study } 1 \text { of } \\
\text { previous } \\
\text { research }\end{array}$ & $\begin{array}{l}\text { replication or } \\
\text { replicate }\end{array}$ & independent & $\begin{array}{l}\text { independent quasi-random } \\
\text { replication }\end{array}$ \\
\hline
\end{tabular}




\begin{tabular}{|c|c|c|c|c|c|c|c|}
\hline \# & Journal & Search & Study & $\begin{array}{l}\text { Which } \\
\text { repetition }\end{array}$ & Terminology & Independence & $\begin{array}{l}\text { Type of replication or } \\
\text { reproducibility study }\end{array}$ \\
\hline 32 & AMJ & full text & $\begin{array}{l}\text { Glaser, Stam, \& } \\
\text { Takeuchi (2016) }\end{array}$ & $\begin{array}{l}\text { study } 1 \text { of } \\
\text { previous } \\
\text { research }\end{array}$ & $\begin{array}{l}\text { replication or } \\
\text { replicate }\end{array}$ & independent & $\begin{array}{l}\text { independent quasi-random } \\
\text { replication }\end{array}$ \\
\hline 33 & AMJ & full text & Grant (2012) & $\begin{array}{l}\text { study } 2 \text { of study } \\
1\end{array}$ & $\begin{array}{l}\text { constructive } \\
\text { replication }\end{array}$ & dependent & $\begin{array}{l}\text { dependent quasi-random } \\
\text { replication }\end{array}$ \\
\hline 34 & AMJ & $\begin{array}{l}\text { abstract } \\
\& \text { title }\end{array}$ & $\begin{array}{l}\text { Grant,Gino, \& } \\
\text { Hofmann (2011) }\end{array}$ & $\begin{array}{l}\text { study } 2 \text { of study } \\
1\end{array}$ & $\begin{array}{l}\text { constructive } \\
\text { replication }\end{array}$ & dependent & $\begin{array}{l}\text { dependent regressive } \\
\text { replication }\end{array}$ \\
\hline 35 & $\mathrm{AMJ}$ & full text & $\begin{array}{l}\text { Grégoire \& } \\
\text { Shepherd (2012) }\end{array}$ & $\begin{array}{l}\text { sample } 2 \text { of } \\
\text { sample } 1\end{array}$ & $\begin{array}{l}\text { replication or } \\
\text { replicate }\end{array}$ & dependent & dependent literal replication \\
\hline 36 & AMJ & full text & $\begin{array}{l}\text { Grégoire \& } \\
\text { Shepherd (2012) }\end{array}$ & footnote 5 & $\begin{array}{l}\text { replication or } \\
\text { replicate }\end{array}$ & independent & $\begin{array}{l}\text { independent quasi-random } \\
\text { replication }\end{array}$ \\
\hline 37 & $\mathrm{AMJ}$ & full text & $\begin{array}{l}\text { Greve \& Zhang } \\
\text { (2017) }\end{array}$ & study 1 & $\begin{array}{l}\text { replication or } \\
\text { replicate }\end{array}$ & dependent & $\begin{array}{l}\text { dependent quasi-random } \\
\text { reproducibility }\end{array}$ \\
\hline 38 & AMJ & full text & $\begin{array}{l}\text { Grohsjean, Kober, } \\
\text { \& Zucchini (2016) }\end{array}$ & study 1 & $\begin{array}{l}\text { replication or } \\
\text { replicate }\end{array}$ & dependent & $\begin{array}{l}\text { dependent quasi-random } \\
\text { reproducibility }\end{array}$ \\
\hline 39 & AMJ & full text & $\begin{array}{l}\text { Hafenbrädl \& } \\
\text { Waeger (2017) }\end{array}$ & $\begin{array}{l}\text { study } 4 \text { of } \\
\text { studies } 1-3\end{array}$ & $\begin{array}{l}\text { replication or } \\
\text { replicate }\end{array}$ & dependent & $\begin{array}{l}\text { dependent quasi-random } \\
\text { replication }\end{array}$ \\
\hline 40 & AMJ & full text & $\begin{array}{l}\text { Hafenbrädl \& } \\
\text { Waeger (2017) }\end{array}$ & study 2 or 1 & nothing & dependent & $\begin{array}{l}\text { dependent confounded } \\
\text { replication }\end{array}$ \\
\hline
\end{tabular}




\begin{tabular}{|c|c|c|c|c|c|c|c|}
\hline \# & Journal & Search & Study & $\begin{array}{l}\text { Which } \\
\text { repetition }\end{array}$ & Terminology & Independence & $\begin{array}{l}\text { Type of replication or } \\
\text { reproducibility study }\end{array}$ \\
\hline 41 & AMJ & full text & $\begin{array}{l}\text { Hafenbrädl \& } \\
\text { Waeger (2017) }\end{array}$ & $\begin{array}{l}\text { study } 3 \text { of } \\
\text { studies } 1 \text { and } 2\end{array}$ & nothing & dependent & generalizability \\
\hline 42 & AMJ & full text & $\begin{array}{l}\text { Harrison \& Wagner } \\
(2016)\end{array}$ & $\begin{array}{l}\text { study } 1 \text { of } \\
\text { previous } \\
\text { research }\end{array}$ & $\begin{array}{l}\text { replication or } \\
\text { replicate }\end{array}$ & independent & $\begin{array}{l}\text { independent quasi-random } \\
\text { replication }\end{array}$ \\
\hline 43 & AMJ & full text & $\begin{array}{l}\text { Heimeriks, } \\
\text { Schijven, \& Gates } \\
(2012)\end{array}$ & study 1 & $\begin{array}{l}\text { replication or } \\
\text { replicate }\end{array}$ & dependent & $\begin{array}{l}\text { dependent quasi-random } \\
\text { reproducibility }\end{array}$ \\
\hline 44 & AMJ & $\begin{array}{l}\text { abstract } \\
\& \text { title }\end{array}$ & $\begin{array}{l}\text { Hewlin, Dumas, \& } \\
\text { Burnett (2017) }\end{array}$ & $\begin{array}{l}\text { study } 2 \text { of study } \\
1\end{array}$ & $\begin{array}{l}\text { replication or } \\
\text { replicate }\end{array}$ & dependent & $\begin{array}{l}\text { dependent quasi-random } \\
\text { replication }\end{array}$ \\
\hline 45 & AMJ & full text & $\begin{array}{l}\text { Jacquart \& } \\
\text { Antonakis (2015) }\end{array}$ & $\begin{array}{l}\text { study } 2 \text { of study } \\
1\end{array}$ & $\begin{array}{l}\text { replication or } \\
\text { replicate }\end{array}$ & dependent & $\begin{array}{l}\text { dependent substantial } \\
\text { constructive replication }\end{array}$ \\
\hline 46 & AMJ & full text & Jia \& Liden (2015) & $\begin{array}{l}\text { study } 2 \text { of } \\
\text { study } 1\end{array}$ & $\begin{array}{l}\text { constructive } \\
\text { replication }\end{array}$ & dependent & $\begin{array}{l}\text { dependent confounded } \\
\text { replication }\end{array}$ \\
\hline 47 & AMJ & full text & $\begin{array}{l}\text { Jones, Willness, \& } \\
\text { Madey (2014) }\end{array}$ & $\begin{array}{l}\text { study } 2 \text { of study } \\
1\end{array}$ & $\begin{array}{l}\text { replication or } \\
\text { replicate }\end{array}$ & dependent & $\begin{array}{l}\text { dependent confounded } \\
\text { replication }\end{array}$ \\
\hline 48 & AMJ & full text & $\begin{array}{l}\text { Kilduff, Galinsky, } \\
\text { Gallo, \& Reade } \\
(2016)\end{array}$ & $\begin{array}{l}\text { study } 3 \text { of } \\
\text { studies } 1 \text { and } 2\end{array}$ & $\begin{array}{l}\text { replication or } \\
\text { replicate }\end{array}$ & dependent & $\begin{array}{l}\text { dependent quasi-random } \\
\text { replication }\end{array}$ \\
\hline
\end{tabular}




\begin{tabular}{|c|c|c|c|c|c|c|c|}
\hline \# & Journal & Search & Study & $\begin{array}{l}\text { Which } \\
\text { repetition }\end{array}$ & Terminology & Independence & $\begin{array}{l}\text { Type of replication or } \\
\text { reproducibility study }\end{array}$ \\
\hline 49 & AMJ & full text & $\begin{array}{l}\text { Kilduff, Galinsky, } \\
\text { Gallo, \& Reade } \\
\text { (2016) }\end{array}$ & study 2 of 1 & nothing & dependent & $\begin{array}{l}\text { dependent quasi-random } \\
\text { replication }\end{array}$ \\
\hline 50 & AMJ & full text & $\begin{array}{l}\text { Kilduff, Galinsky, } \\
\text { Gallo, \& Reade } \\
\text { (2016) }\end{array}$ & $\begin{array}{l}\text { study } 4 \text { of } \\
\text { studies } 1-3\end{array}$ & nothing & dependent & $\begin{array}{l}\text { dependent quasi-random } \\
\text { replication }\end{array}$ \\
\hline 51 & AMJ & full text & $\begin{array}{l}\text { Klingebiel \& Adner } \\
(2015)\end{array}$ & study 1 & $\begin{array}{l}\text { replication or } \\
\text { replicate }\end{array}$ & dependent & $\begin{array}{l}\text { dependent quasi-random } \\
\text { reproducibility }\end{array}$ \\
\hline 52 & AMJ & full text & $\begin{array}{l}\text { Koopman, Lanaj, \& } \\
\text { Scott (2016) }\end{array}$ & $\begin{array}{l}\text { study } 1 \text { of } \\
\text { previous } \\
\text { research }\end{array}$ & $\begin{array}{l}\text { constructive } \\
\text { replication }\end{array}$ & independent & $\begin{array}{l}\text { independent quasi-random } \\
\text { replication }\end{array}$ \\
\hline 53 & AMJ & full text & $\begin{array}{l}\text { Kozhikode \& Li } \\
(2012)\end{array}$ & study 1 & $\begin{array}{l}\text { replication or } \\
\text { replicate }\end{array}$ & dependent & $\begin{array}{l}\text { dependent quasi-random } \\
\text { reproducibility }\end{array}$ \\
\hline 54 & AMJ & full text & $\begin{array}{l}\text { Kraimer, Shaffer, } \\
\text { Harrison, \& Ren } \\
(2012)\end{array}$ & study 1 & $\begin{array}{l}\text { replication or } \\
\text { replicate }\end{array}$ & dependent & $\begin{array}{l}\text { dependent quasi-random } \\
\text { reproducibility }\end{array}$ \\
\hline 55 & AMJ & full text & $\begin{array}{l}\text { Lam, Xu, \& Chan } \\
(2015)\end{array}$ & $\begin{array}{l}\text { study } 2 \text { of study } \\
1\end{array}$ & $\begin{array}{l}\text { replication or } \\
\text { replicate }\end{array}$ & dependent & $\begin{array}{l}\text { dependent incremental } \\
\text { constructive replication }\end{array}$ \\
\hline 56 & AMJ & full text & $\begin{array}{l}\text { Lanzolla \& } \\
\text { Frankort (2016) }\end{array}$ & study 1 & $\begin{array}{l}\text { replication or } \\
\text { replicate }\end{array}$ & dependent & $\begin{array}{l}\text { dependent quasi-random } \\
\text { reproducibility }\end{array}$ \\
\hline
\end{tabular}




\begin{tabular}{|c|c|c|c|c|c|c|c|}
\hline \# & Journal & Search & Study & $\begin{array}{l}\text { Which } \\
\text { repetition }\end{array}$ & Terminology & Independence & $\begin{array}{l}\text { Type of replication or } \\
\text { reproducibility study }\end{array}$ \\
\hline 57 & AMJ & $\begin{array}{l}\text { abstract } \\
\& \text { title }\end{array}$ & $\begin{array}{l}\text { Lee, Pitesa, Pillutla, } \\
\text { \& Thau (2017) }\end{array}$ & $\begin{array}{l}\text { study } 2 \text { of study } \\
1\end{array}$ & $\begin{array}{l}\text { replication or } \\
\text { replicate }\end{array}$ & dependent & $\begin{array}{l}\text { dependent quasi-random } \\
\text { replication }\end{array}$ \\
\hline 58 & AMJ & $\begin{array}{l}\text { abstract } \\
\& \text { title }\end{array}$ & $\begin{array}{l}\text { Lee, Pitesa, Pillutla, } \\
\& \text { Thau (2017) }\end{array}$ & $\begin{array}{l}\text { study } 3 \text { of } \\
\text { studies } 1 \text { and } 2\end{array}$ & $\begin{array}{l}\text { replication or } \\
\text { replicate }\end{array}$ & dependent & $\begin{array}{l}\text { dependent incremental } \\
\text { constructive replication }\end{array}$ \\
\hline 59 & AMJ & full text & $\begin{array}{l}\text { Lee, Pitesa, Thau, } \\
\text { \& Pillutla (2015) }\end{array}$ & $\begin{array}{l}\text { study } 3 \text { of } \\
\text { studies } 1 \text { and } 2\end{array}$ & $\begin{array}{l}\text { constructive } \\
\text { replication }\end{array}$ & dependent & $\begin{array}{l}\text { dependent quasi-random } \\
\text { replication }\end{array}$ \\
\hline 60 & AMJ & full text & $\begin{array}{l}\text { Lee, Pitesa, Thau, } \\
\text { \& Pillutla (2015) }\end{array}$ & $\begin{array}{l}\text { study } 2 \text { of study } \\
1\end{array}$ & $\begin{array}{l}\text { constructive } \\
\text { replication }\end{array}$ & dependent & $\begin{array}{l}\text { dependent quasi-random } \\
\text { replication }\end{array}$ \\
\hline 61 & AMJ & $\begin{array}{l}\text { abstract } \\
\& \text { title }\end{array}$ & $\begin{array}{l}\text { Lepine, Zhang, } \\
\text { Crawford, \& Rich } \\
\text { (2016) }\end{array}$ & $\begin{array}{l}\text { study } 2 \text { of study } \\
1\end{array}$ & $\begin{array}{l}\text { replication or } \\
\text { replicate }\end{array}$ & dependent & $\begin{array}{l}\text { dependent incremental } \\
\text { constructive replication }\end{array}$ \\
\hline 62 & AMJ & full text & $\begin{array}{l}\text { Leslie, Manchester, } \\
\text { \& Dahm (2017) }\end{array}$ & $\begin{array}{l}\text { study } 4 \text { of } \\
\text { studies } 1-3\end{array}$ & nothing & dependent & $\begin{array}{l}\text { dependent quasi-random } \\
\text { replication }\end{array}$ \\
\hline 63 & AMJ & full text & $\begin{array}{l}\text { Leslie, Manchester, } \\
\text { \& Dahm (2017) }\end{array}$ & $\begin{array}{l}\text { study } 2 \text { of study } \\
1\end{array}$ & nothing & dependent & $\begin{array}{l}\text { dependent confounded } \\
\text { replication }\end{array}$ \\
\hline 64 & AMJ & full text & $\begin{array}{l}\text { Leslie, Manchester, } \\
\text { \& Dahm (2017) }\end{array}$ & $\begin{array}{l}\text { study } 1 \text { of } \\
\text { previous } \\
\text { research }\end{array}$ & $\begin{array}{l}\text { replication or } \\
\text { replicate }\end{array}$ & independent & $\begin{array}{l}\text { independent quasi-random } \\
\text { replication }\end{array}$ \\
\hline 65 & AMJ & full text & $\begin{array}{l}\text { Leslie, Manchester, } \\
\text { \& Dahm (2017) }\end{array}$ & $\begin{array}{l}\text { study } 3 \text { of } \\
\text { previous } \\
\text { research }\end{array}$ & $\begin{array}{l}\text { replication or } \\
\text { replicate }\end{array}$ & independent & $\begin{array}{l}\text { independent quasi-random } \\
\text { reproducibility }\end{array}$ \\
\hline
\end{tabular}




\begin{tabular}{|c|c|c|c|c|c|c|c|}
\hline \# & Journal & Search & Study & $\begin{array}{l}\text { Which } \\
\text { repetition }\end{array}$ & Terminology & Independence & $\begin{array}{l}\text { Type of replication or } \\
\text { reproducibility study }\end{array}$ \\
\hline 66 & AMJ & full text & $\begin{array}{l}\text { Lin, Law, \& Jing } \\
(2017)\end{array}$ & $\begin{array}{l}\text { study } 2 \text { of study } \\
1\end{array}$ & $\begin{array}{l}\text { constructive } \\
\text { replication }\end{array}$ & dependent & $\begin{array}{l}\text { dependent quasi-random } \\
\text { replication }\end{array}$ \\
\hline 67 & AMJ & full text & $\begin{array}{l}\text { Little, Smith Major, } \\
\text { Hinojosa, \& Nelson } \\
(2015)\end{array}$ & $\begin{array}{l}\text { sample } 2 \text { aof } \\
\text { sample } 1\end{array}$ & $\begin{array}{l}\text { replication or } \\
\text { replicate }\end{array}$ & dependent & dependent literal replication \\
\hline 68 & AMJ & full text & $\begin{array}{l}\text { Lopez-Kidwell, } \\
\text { Grosser, Dineen, \& } \\
\text { Borgatti (2013) }\end{array}$ & $\begin{array}{l}\text { study } 2 \text { of study } \\
1\end{array}$ & $\begin{array}{l}\text { replication or } \\
\text { replicate }\end{array}$ & dependent & $\begin{array}{l}\text { dependent substantial } \\
\text { constructive replication }\end{array}$ \\
\hline 69 & $\mathrm{AMJ}$ & full text & $\begin{array}{l}\text { Maltarich, Nyberg, } \\
\text { Reilly, Abdulsalam, } \\
\text { \& Martin (2017) }\end{array}$ & study 1 & $\begin{array}{l}\text { replication or } \\
\text { replicate }\end{array}$ & $\begin{array}{l}\text { semi- } \\
\text { independent }\end{array}$ & $\begin{array}{l}\text { semi-independent quasi- } \\
\text { random replication }\end{array}$ \\
\hline 70 & $\mathrm{AMJ}$ & $\begin{array}{l}\text { abstract } \\
\& \text { title }\end{array}$ & $\begin{array}{l}\text { Matta, Scott, } \\
\text { Colquitt, Koopman, } \\
\text { \& Passantino } \\
(2017)\end{array}$ & $\begin{array}{l}\text { study } 2 \text { of study } \\
1 \text { and footnote } 8\end{array}$ & $\begin{array}{l}\text { replicate and } \\
\text { extend }\end{array}$ & dependent & $\begin{array}{l}\text { dependent confounded } \\
\text { replication }\end{array}$ \\
\hline 71 & AMJ & $\begin{array}{l}\text { abstract } \\
\& \text { title }\end{array}$ & $\begin{array}{l}\text { Mawritz, } \\
\text { Greenbaum, Butts, } \\
\text { \& Graham (2017) }\end{array}$ & $\begin{array}{l}\text { study } 2 \text { of study } \\
1\end{array}$ & $\begin{array}{l}\text { replication or } \\
\text { replicate }\end{array}$ & dependent & $\begin{array}{l}\text { dependent quasi-random } \\
\text { replication }\end{array}$ \\
\hline 72 & AMJ & full text & $\begin{array}{l}\text { Opper, Nee, \& } \\
\text { Holm (2017) }\end{array}$ & Appendix F & $\begin{array}{l}\text { replication or } \\
\text { replicate }\end{array}$ & dependent & $\begin{array}{l}\text { no replication or } \\
\text { reproducibility study }\end{array}$ \\
\hline
\end{tabular}




\begin{tabular}{|c|c|c|c|c|c|c|c|}
\hline \# & Journal & Search & Study & $\begin{array}{l}\text { Which } \\
\text { repetition }\end{array}$ & Terminology & Independence & $\begin{array}{l}\text { Type of replication or } \\
\text { reproducibility study }\end{array}$ \\
\hline 73 & AMJ & full text & $\begin{array}{l}\text { Ou, Seo, Choi, \& } \\
\text { Hom (2017) }\end{array}$ & & $\begin{array}{l}\text { constructive } \\
\text { replication }\end{array}$ & independent & generalizability \\
\hline 74 & AMJ & full text & $\begin{array}{l}\text { Owens \& Hekman } \\
(2016)\end{array}$ & $\begin{array}{l}\text { study } 3 \text { of study } \\
1 \text { and } 2\end{array}$ & $\begin{array}{l}\text { replication or } \\
\text { replicate }\end{array}$ & dependent & $\begin{array}{l}\text { dependent confounded } \\
\text { replication }\end{array}$ \\
\hline 75 & AMJ & full text & $\begin{array}{l}\text { Owens \& Hekman } \\
(2016)\end{array}$ & $\begin{array}{l}\text { study } 2 \text { of study } \\
1\end{array}$ & nothing & dependent & $\begin{array}{l}\text { dependent quasi-random } \\
\text { replication }\end{array}$ \\
\hline 76 & AMJ & full text & $\begin{array}{l}\text { Parker, Johnson, } \\
\text { Collins, \& Nguyen } \\
\text { (2013) }\end{array}$ & study 1 & $\begin{array}{l}\text { replication or } \\
\text { replicate }\end{array}$ & dependent & $\begin{array}{l}\text { dependent quasi-random } \\
\text { reproducibility }\end{array}$ \\
\hline 77 & AMJ & full text & $\begin{array}{l}\text { Patel, Messersmith, } \\
\text { \& Lepak (2013) }\end{array}$ & study 1 & $\begin{array}{l}\text { replication or } \\
\text { replicate }\end{array}$ & dependent & $\begin{array}{l}\text { dependent quasi-random } \\
\text { reproducibility }\end{array}$ \\
\hline 78 & AMJ & full text & $\begin{array}{l}\text { Pitesa \& Thau } \\
(2013)\end{array}$ & $\begin{array}{l}\text { study } 2 \text { of study } \\
1\end{array}$ & $\begin{array}{l}\text { constructive } \\
\text { replication }\end{array}$ & dependent & $\begin{array}{l}\text { dependent quasi-random } \\
\text { replication }\end{array}$ \\
\hline 79 & AMJ & full text & $\begin{array}{l}\text { Pitesa \& Thau } \\
\text { (2013) }\end{array}$ & $\begin{array}{l}\text { study } 3 \text { of study } \\
1 \text { and } 2\end{array}$ & $\begin{array}{l}\text { constructive } \\
\text { replication }\end{array}$ & independent & generalizability \\
\hline 80 & AMJ & full text & $\begin{array}{l}\text { Raffiee \& Coff } \\
(2016)\end{array}$ & $\begin{array}{l}\text { sample } 2 \text { of } \\
\text { sample } 1\end{array}$ & $\begin{array}{l}\text { replication or } \\
\text { replicate }\end{array}$ & dependent & generalizability \\
\hline 81 & $\mathrm{AMJ}$ & full text & $\begin{array}{l}\text { Raffiee \& Feng } \\
(2014)\end{array}$ & $\begin{array}{l}\text { study } 1 \text { of } \\
\text { previous } \\
\text { research }\end{array}$ & $\begin{array}{l}\text { replication or } \\
\text { replicate }\end{array}$ & independent & $\begin{array}{l}\text { independent quasi-random } \\
\text { replication }\end{array}$ \\
\hline
\end{tabular}




\begin{tabular}{|c|c|c|c|c|c|c|c|}
\hline \# & Journal & Search & Study & $\begin{array}{l}\text { Which } \\
\text { repetition }\end{array}$ & Terminology & Independence & $\begin{array}{l}\text { Type of replication or } \\
\text { reproducibility study }\end{array}$ \\
\hline 82 & AMJ & $\begin{array}{l}\text { abstract } \\
\& \text { title }\end{array}$ & $\begin{array}{l}\text { Ramarajan, } \\
\text { Rothbard, \& Wilk } \\
(2017)\end{array}$ & $\begin{array}{l}\text { study } 2 \mathrm{~b} \text { of } \\
\text { studies } 1 \text { and } 2 \mathrm{a}\end{array}$ & $\begin{array}{l}\text { constructive } \\
\text { replication }\end{array}$ & dependent & $\begin{array}{l}\text { dependent quasi-random } \\
\text { replication }\end{array}$ \\
\hline 83 & AMJ & $\begin{array}{l}\text { abstract } \\
\& \text { title }\end{array}$ & $\begin{array}{l}\text { Ramarajan, } \\
\text { Rothbard, \& Wilk } \\
(2017)\end{array}$ & $\begin{array}{l}\text { study } 2 \mathrm{a} \text { of } \\
\text { study } 1\end{array}$ & $\begin{array}{l}\text { constructive } \\
\text { replication }\end{array}$ & dependent & $\begin{array}{l}\text { dependent confounded } \\
\text { replication }\end{array}$ \\
\hline 84 & AMJ & full text & $\begin{array}{l}\text { Reyt \& Wiesenfeld } \\
(2015)\end{array}$ & $\begin{array}{l}\text { study } 2 \text { of study } \\
1\end{array}$ & $\begin{array}{l}\text { replication or } \\
\text { replicate }\end{array}$ & dependent & $\begin{array}{l}\text { dependent regressive } \\
\text { replication }\end{array}$ \\
\hline 85 & AMJ & full text & $\begin{array}{l}\text { Reyt \& Wiesenfeld } \\
(2015)\end{array}$ & $\begin{array}{l}\text { study } 4 \text { of } \\
\text { studies } 1-3\end{array}$ & $\begin{array}{l}\text { replicate and } \\
\text { extend }\end{array}$ & dependent & $\begin{array}{l}\text { dependent confounded } \\
\text { replication }\end{array}$ \\
\hline 86 & $\mathrm{AMJ}$ & full text & Rodell (2013) & study 2 of 1 & $\begin{array}{l}\text { replication or } \\
\text { replicate }\end{array}$ & dependent & $\begin{array}{l}\text { dependent incremental } \\
\text { constructive replication }\end{array}$ \\
\hline 87 & AMJ & full text & $\begin{array}{l}\text { Schaubroeck, Peng, } \\
\text { \& Hannah (2013) }\end{array}$ & study 1 & $\begin{array}{l}\text { replication or } \\
\text { replicate }\end{array}$ & dependent & $\begin{array}{l}\text { dependent quasi-random } \\
\text { reproducibility }\end{array}$ \\
\hline 88 & AMJ & full text & Shani \& Westphal & study 1 & $\begin{array}{l}\text { replication or } \\
\text { replicate }\end{array}$ & $\begin{array}{l}\text { semi- } \\
\text { independent }\end{array}$ & $\begin{array}{l}\text { semi-independent quasi- } \\
\text { random replication }\end{array}$ \\
\hline 89 & AMJ & $\begin{array}{l}\text { abstract } \\
\& \text { title }\end{array}$ & $\begin{array}{l}\text { Shaw, Dineen, } \\
\text { Fang, \& Vellella } \\
(2009)\end{array}$ & $\begin{array}{l}\text { study } 2 \text { of study } \\
1\end{array}$ & $\begin{array}{l}\text { constructive } \\
\text { replication }\end{array}$ & dependent & $\begin{array}{l}\text { dependent quasi-random } \\
\text { replication }\end{array}$ \\
\hline 90 & AMJ & full text & $\begin{array}{l}\text { Sirola \& Pitesa } \\
(2017)\end{array}$ & $\begin{array}{l}\text { study } 4 \text { of } \\
\text { studies } 1-3\end{array}$ & $\begin{array}{l}\text { conceptual } \\
\text { replication }\end{array}$ & dependent & $\begin{array}{l}\text { dependent quasi-random } \\
\text { replication }\end{array}$ \\
\hline
\end{tabular}




\begin{tabular}{|c|c|c|c|c|c|c|c|}
\hline \# & Journal & Search & Study & $\begin{array}{l}\text { Which } \\
\text { repetition }\end{array}$ & Terminology & Independence & $\begin{array}{l}\text { Type of replication or } \\
\text { reproducibility study }\end{array}$ \\
\hline 91 & AMJ & full text & $\begin{array}{l}\text { Sirola \& Pitesa } \\
(2017)\end{array}$ & $\begin{array}{l}\text { study } 3 \text { of } \\
\text { studies } 1 \text { or } 2\end{array}$ & $\begin{array}{l}\text { conceptual } \\
\text { replication }\end{array}$ & dependent & $\begin{array}{l}\text { dependent incremental } \\
\text { constructive replication }\end{array}$ \\
\hline 92 & AMJ & full text & $\begin{array}{l}\text { Sirola \& Pitesa } \\
(2017)\end{array}$ & $\begin{array}{l}\text { study } 2 \text { of study } \\
1\end{array}$ & $\begin{array}{l}\text { conceptual } \\
\text { replication }\end{array}$ & $\begin{array}{l}\text { semi- } \\
\text { independent }\end{array}$ & $\begin{array}{l}\text { semi-independent quasi- } \\
\text { random replication }\end{array}$ \\
\hline 93 & AMJ & full text & $\begin{array}{l}\text { Soenen, Melkonian, } \\
\text { \& Ambrose (2017) }\end{array}$ & $\begin{array}{l}\text { footnote } 3 \text { of } \\
\text { study } 1\end{array}$ & $\begin{array}{l}\text { replication or } \\
\text { replicate }\end{array}$ & dependent & $\begin{array}{l}\text { dependent quasi-random } \\
\text { replication }\end{array}$ \\
\hline 94 & AMJ & full text & $\begin{array}{l}\text { Stuart \& Moore } \\
(2017)\end{array}$ & study 1 & $\begin{array}{l}\text { replication or } \\
\text { replicate }\end{array}$ & dependent & $\begin{array}{l}\text { dependent quasi-random } \\
\text { reproducibility }\end{array}$ \\
\hline 95 & AMJ & full text & $\begin{array}{l}\text { Summers, } \\
\text { Humphrey, \& } \\
\text { Ferris (2012) }\end{array}$ & footnote 2 & $\begin{array}{l}\text { replication or } \\
\text { replicate }\end{array}$ & dependent & $\begin{array}{l}\text { dependent quasi-random } \\
\text { reproducibility }\end{array}$ \\
\hline 96 & AMJ & full text & $\begin{array}{l}\text { Tost, Gino, \& } \\
\text { Larrick. (2013) }\end{array}$ & study 2 of 1 & $\begin{array}{l}\text { replicate and } \\
\text { extend }\end{array}$ & dependent & generalizability \\
\hline 97 & AMJ & full text & $\begin{array}{l}\text { Tost, Gino, \& } \\
\text { Larrick. (2013) }\end{array}$ & $\begin{array}{l}\text { study } 3 \text { of } 2 \text { and } \\
1\end{array}$ & $\begin{array}{l}\text { replicate and } \\
\text { extend }\end{array}$ & dependent & generalizability \\
\hline 98 & AMJ & full text & $\begin{array}{l}\text { Trevor, Reilly, \& } \\
\text { Gerhart (2012) }\end{array}$ & & $\begin{array}{l}\text { replication or } \\
\text { replicate }\end{array}$ & dependent & $\begin{array}{l}\text { dependent quasi-random } \\
\text { reproducibility }\end{array}$ \\
\hline 99 & $\mathrm{AMJ}$ & full text & $\begin{array}{l}\text { Vakili \& McGahan } \\
\text { (2016) }\end{array}$ & & $\begin{array}{l}\text { replication or } \\
\text { replicate }\end{array}$ & dependent & $\begin{array}{l}\text { dependent quasi-random } \\
\text { reproducibility }\end{array}$ \\
\hline
\end{tabular}




\begin{tabular}{|c|c|c|c|c|c|c|c|}
\hline \# & Journal & Search & Study & $\begin{array}{l}\text { Which } \\
\text { repetition }\end{array}$ & Terminology & Independence & $\begin{array}{l}\text { Type of replication or } \\
\text { reproducibility study }\end{array}$ \\
\hline 100 & AMJ & full text & $\begin{array}{l}\text { Vincent \& } \\
\text { Kouchaki (2016) }\end{array}$ & $\begin{array}{l}\text { study } 2 \text { of study } \\
1\end{array}$ & $\begin{array}{l}\text { replicate and } \\
\text { extend }\end{array}$ & dependent & $\begin{array}{l}\text { no replication or } \\
\text { reproducibility study }\end{array}$ \\
\hline 101 & AMJ & full text & $\begin{array}{l}\text { Vincent \& } \\
\text { Kouchaki (2016) }\end{array}$ & $\begin{array}{l}\text { study } 4 \text { of } \\
\text { studies } 1-3\end{array}$ & nothing & dependent & $\begin{array}{l}\text { dependent quasi-random } \\
\text { replication }\end{array}$ \\
\hline 102 & AMJ & full text & $\begin{array}{l}\text { Vincent \& } \\
\text { Kouchaki (2016) }\end{array}$ & $\begin{array}{l}\text { study } 5 \text { of } \\
\text { studies } 3 \text { and } 4\end{array}$ & nothing & dependent & $\begin{array}{l}\text { dependent confounded } \\
\text { replication }\end{array}$ \\
\hline 103 & AMJ & full text & $\begin{array}{l}\text { Walker, Bauer, } \\
\text { Cole,Bernerth, } \\
\text { Feild, \& Short } \\
\text { (2013) }\end{array}$ & $\begin{array}{l}\text { study } 2 \text { of study } \\
1\end{array}$ & $\begin{array}{l}\text { replication or } \\
\text { replicate }\end{array}$ & dependent & $\begin{array}{l}\text { dependent confounded } \\
\text { replication }\end{array}$ \\
\hline 104 & AMJ & full text & $\begin{array}{l}\text { Wang, Singh, Li, } \\
\text { Mishra, Ambrose, } \\
\text { \& Biernat (2017) }\end{array}$ & study 2 of study & $\begin{array}{l}\text { replication or } \\
\text { replicate }\end{array}$ & dependent & $\begin{array}{l}\text { dependent confounded } \\
\text { replication }\end{array}$ \\
\hline 105 & AMJ & $\begin{array}{l}\text { abstract } \\
\& \text { title }\end{array}$ & $\begin{array}{l}\text { Wee, Liao, Liu, \& } \\
\text { Liu (2017) }\end{array}$ & $\begin{array}{l}\text { study } 2 \text { of study } \\
1\end{array}$ & $\begin{array}{l}\text { replication or } \\
\text { replicate }\end{array}$ & dependent & $\begin{array}{l}\text { dependent quasi-random } \\
\text { replication }\end{array}$ \\
\hline 106 & AMJ & full text & $\begin{array}{l}\text { Wo, Ambrose, \& } \\
\text { Schminke (2015) }\end{array}$ & study 2 of 1 & $\begin{array}{l}\text { constructive } \\
\text { replication }\end{array}$ & dependent & $\begin{array}{l}\text { dependent substantial } \\
\text { constructive replication }\end{array}$ \\
\hline 107 & $\mathrm{AMJ}$ & full text & $\begin{array}{l}\text { Wombacher \& } \\
\text { Felfe (2017) }\end{array}$ & $\begin{array}{l}\text { study } 2 \text { of study } \\
1\end{array}$ & $\begin{array}{l}\text { replication or } \\
\text { replicate }\end{array}$ & dependent & $\begin{array}{l}\text { dependent confounded } \\
\text { replication }\end{array}$ \\
\hline 108 & AMJ & full text & $\begin{array}{l}\text { Yam, Klotz, He, \& } \\
\text { Reynolds (2017) }\end{array}$ & $\begin{array}{l}\text { study } 2 \text { of study } \\
1\end{array}$ & $\begin{array}{l}\text { constructive } \\
\text { replication }\end{array}$ & dependent & generalizability \\
\hline
\end{tabular}




\begin{tabular}{|c|c|c|c|c|c|c|c|}
\hline \# & Journal & Search & Study & $\begin{array}{l}\text { Which } \\
\text { repetition }\end{array}$ & Terminology & Independence & $\begin{array}{l}\text { Type of replication or } \\
\text { reproducibility study }\end{array}$ \\
\hline 109 & AMJ & $\begin{array}{l}\text { abstract } \\
\& \text { title }\end{array}$ & $\begin{array}{l}\text { Zapata, Carton, \& } \\
\text { Liu (2016) }\end{array}$ & $\begin{array}{l}\text { study } 2 \text { of study } \\
1\end{array}$ & $\begin{array}{l}\text { replication or } \\
\text { replicate }\end{array}$ & dependent & $\begin{array}{l}\text { dependent quasi-random } \\
\text { replication }\end{array}$ \\
\hline 110 & AMJ & full text & Zhang \& Qu (2016) & footnote 12 & $\begin{array}{l}\text { replication or } \\
\text { replicate }\end{array}$ & dependent & generalizability \\
\hline 111 & AMJ & full text & Zhao \& Wry (2016) & study 1 & $\begin{array}{l}\text { replication or } \\
\text { replicate }\end{array}$ & dependent & $\begin{array}{l}\text { dependent quasi-random } \\
\text { reproducibility }\end{array}$ \\
\hline 112 & JAP & full text & $\begin{array}{l}\text { Adam \& Shirako } \\
(2013)\end{array}$ & $\begin{array}{l}\text { study } 3 \text { of } 2 \text { and } \\
1\end{array}$ & $\begin{array}{l}\text { replication or } \\
\text { replicate }\end{array}$ & dependent & $\begin{array}{l}\text { dependent incremental } \\
\text { constructive replication }\end{array}$ \\
\hline 113 & JAP & full text & $\begin{array}{l}\text { Adam \& Shirako } \\
(2013)\end{array}$ & $\begin{array}{l}\text { study } 2 \text { of study } \\
1\end{array}$ & $\begin{array}{l}\text { replication or } \\
\text { replicate }\end{array}$ & dependent & $\begin{array}{l}\text { dependent substantial } \\
\text { constructive replication }\end{array}$ \\
\hline 114 & JAP & full text & $\begin{array}{l}\text { Adam \& Shirako } \\
(2013)\end{array}$ & $\begin{array}{l}\text { study } 4 \text { of } \\
\text { studies } 1,2,3\end{array}$ & $\begin{array}{l}\text { replication or } \\
\text { replicate }\end{array}$ & dependent & generalizability \\
\hline 115 & JAP & full text & $\begin{array}{l}\text { Ali, Lyons, \& Ryan } \\
\text { (2017) }\end{array}$ & $\begin{array}{l}\text { study } 2 \text { of study } \\
1\end{array}$ & $\begin{array}{l}\text { replication or } \\
\text { replicate }\end{array}$ & dependent & $\begin{array}{l}\text { dependent incremental } \\
\text { constructive replication }\end{array}$ \\
\hline 116 & JAP & full text & $\begin{array}{l}\text { Ali, Ryan, Lyons, } \\
\text { Ehrhart, \& Wessel } \\
(2016)\end{array}$ & $\begin{array}{l}\text { Study } 3 \text { of } \\
\text { Study } 2\end{array}$ & $\begin{array}{l}\text { replication or } \\
\text { replicate }\end{array}$ & dependent & $\begin{array}{l}\text { dependent incremental } \\
\text { constructive replication }\end{array}$ \\
\hline 117 & JAP & full text & $\begin{array}{l}\text { Ali, Ryan, Lyons, } \\
\text { Ehrhart, \& Wessel } \\
\text { (2016) }\end{array}$ & study 2 of 1 & $\begin{array}{l}\text { replication or } \\
\text { replicate }\end{array}$ & dependent & $\begin{array}{l}\text { dependent incremental } \\
\text { constructive replication }\end{array}$ \\
\hline
\end{tabular}




\begin{tabular}{|c|c|c|c|c|c|c|c|}
\hline \# & Journal & Search & Study & $\begin{array}{l}\text { Which } \\
\text { repetition }\end{array}$ & Terminology & Independence & $\begin{array}{l}\text { Type of replication or } \\
\text { reproducibility study }\end{array}$ \\
\hline 118 & JAP & full text & $\begin{array}{l}\text { Allen, Peltokorpi, } \\
\text { \& Rubenstein } \\
(2016)\end{array}$ & $\begin{array}{l}\text { Study } 2 \text { and } \\
\text { Study } 1\end{array}$ & $\begin{array}{l}\text { constructive } \\
\text { replication }\end{array}$ & dependent & generalizability \\
\hline 119 & JAP & full text & $\begin{array}{l}\text { Arthur, Glaze, } \\
\text { Jarrett, White, } \\
\text { Schurig, \& Taylor } \\
(2014)\end{array}$ & $\begin{array}{l}\text { study in } \\
\text { supplementary } \\
\text { materials as rep } \\
\text { of study in } \\
\text { paper }\end{array}$ & $\begin{array}{l}\text { constructive } \\
\text { replication }\end{array}$ & dependent & $\begin{array}{l}\text { dependent confounded } \\
\text { replication }\end{array}$ \\
\hline 120 & JAP & full text & $\begin{array}{l}\text { Avery, McKay, \& } \\
\text { Volpone (2016) }\end{array}$ & study 2 of study & $\begin{array}{l}\text { constructive } \\
\text { replication }\end{array}$ & dependent & $\begin{array}{l}\text { dependent confounded } \\
\text { replication }\end{array}$ \\
\hline 121 & JAP & full text & $\begin{array}{l}\text { Ballard, Yeo, Loft, } \\
\text { Vancouver, \& Neal } \\
(2016)\end{array}$ & study 1 & $\begin{array}{l}\text { replication or } \\
\text { replicate }\end{array}$ & independent & $\begin{array}{l}\text { independent quasi-random } \\
\text { replication }\end{array}$ \\
\hline 122 & JAP & full text & $\begin{array}{l}\text { Ballinger, Cross, \& } \\
\text { Holtom (2016) }\end{array}$ & study 1 & $\begin{array}{l}\text { replication or } \\
\text { replicate }\end{array}$ & $\begin{array}{l}\text { semi- } \\
\text { independent }\end{array}$ & $\begin{array}{l}\text { semi-independent quasi- } \\
\text { random replication }\end{array}$ \\
\hline 123 & JAP & full text & $\begin{array}{l}\text { Barnes, Reb, \& } \\
\text { Ang (2012) }\end{array}$ & study 1 & $\begin{array}{l}\text { replication or } \\
\text { replicate }\end{array}$ & independent & $\begin{array}{l}\text { independent quasi-random } \\
\text { replication }\end{array}$ \\
\hline 124 & JAP & full text & $\begin{array}{l}\text { Beatty, Barratt, } \\
\text { Berry, \& Sackett } \\
(2014)\end{array}$ & $\begin{array}{l}\text { Study } 2 \text { of study } \\
1\end{array}$ & $\begin{array}{l}\text { replication or } \\
\text { replicate }\end{array}$ & dependent & $\begin{array}{l}\text { dependent quasi-random } \\
\text { reproducibility }\end{array}$ \\
\hline
\end{tabular}




\begin{tabular}{|c|c|c|c|c|c|c|c|}
\hline \# & Journal & Search & Study & $\begin{array}{l}\text { Which } \\
\text { repetition }\end{array}$ & Terminology & Independence & $\begin{array}{l}\text { Type of replication or } \\
\text { reproducibility study }\end{array}$ \\
\hline 125 & JAP & full text & $\begin{array}{l}\text { Beck \& Schmidt } \\
(2013)\end{array}$ & Study 1 & $\begin{array}{l}\text { replication or } \\
\text { replicate }\end{array}$ & independent & $\begin{array}{l}\text { independent quasi-random } \\
\text { replication }\end{array}$ \\
\hline 126 & JAP & full text & $\begin{array}{l}\text { Beck, Scholer, \& } \\
\text { Hughes (2017) }\end{array}$ & $\begin{array}{l}\text { study } 2 \text { of study } \\
1\end{array}$ & nothing & dependent & $\begin{array}{l}\text { dependent incremental } \\
\text { constructive replication }\end{array}$ \\
\hline 127 & JAP & full text & $\begin{array}{l}\text { Belmi \& Pfeffer } \\
(2016)\end{array}$ & $\begin{array}{l}\text { study } 5 \text { of } \\
\text { studies } 3 \text { and } 4\end{array}$ & $\begin{array}{l}\text { conceptual } \\
\text { replication }\end{array}$ & dependent & $\begin{array}{l}\text { dependent quasi-random } \\
\text { replication }\end{array}$ \\
\hline 128 & JAP & full text & $\begin{array}{l}\text { Belmi \& Pfeffer } \\
(2016)\end{array}$ & study $2 \mathrm{~A}$ of 1 & nothing & dependent & generalizability \\
\hline 129 & JAP & full text & $\begin{array}{l}\text { Belmi \& Pfeffer } \\
(2016)\end{array}$ & study $2 \mathrm{~B}$ of $2 \mathrm{~A}$ & nothing & dependent & generalizability \\
\hline 130 & JAP & full text & $\begin{array}{l}\text { Belmi \& Pfeffer } \\
(2016)\end{array}$ & $\begin{array}{l}\text { study } 4 \text { of study } \\
3\end{array}$ & nothing & dependent & generalizability \\
\hline 131 & JAP & $\begin{array}{l}\text { abstract } \\
\text { \& title }\end{array}$ & $\begin{array}{l}\text { Bennett, Gabriel, } \\
\text { Calderwood, } \\
\text { Dahling, \& } \\
\text { Trougakos (2016) }\end{array}$ & $\begin{array}{l}\text { Study } 2 \text { of study } \\
1\end{array}$ & $\begin{array}{l}\text { replication or } \\
\text { replicate }\end{array}$ & dependent & $\begin{array}{l}\text { dependent substantial } \\
\text { constructive replication }\end{array}$ \\
\hline 132 & JAP & $\begin{array}{l}\text { abstract } \\
\text { \& title }\end{array}$ & $\begin{array}{l}\text { Berry, Cullen, \& } \\
\text { Meyer (2014) }\end{array}$ & $\begin{array}{l}\text { Study } 4 \text { of } \\
\text { previous } \\
\text { research }\end{array}$ & $\begin{array}{l}\text { replication or } \\
\text { replicate }\end{array}$ & dependent & $\begin{array}{l}\text { dependent incremental } \\
\text { constructive replication }\end{array}$ \\
\hline
\end{tabular}




\begin{tabular}{|c|c|c|c|c|c|c|c|}
\hline \# & Journal & Search & Study & $\begin{array}{l}\text { Which } \\
\text { repetition }\end{array}$ & Terminology & Independence & $\begin{array}{l}\text { Type of replication or } \\
\text { reproducibility study }\end{array}$ \\
\hline 133 & JAP & $\begin{array}{l}\text { abstract } \\
\& \text { title }\end{array}$ & $\begin{array}{l}\text { Berry, Cullen, \& } \\
\text { Meyer (2014) }\end{array}$ & $\begin{array}{l}\text { Study } 3 \text { of } \\
\text { previous } \\
\text { research }\end{array}$ & $\begin{array}{l}\text { replication or } \\
\text { replicate }\end{array}$ & dependent & $\begin{array}{l}\text { dependent confounded } \\
\text { replication }\end{array}$ \\
\hline 134 & JAP & $\begin{array}{l}\text { abstract } \\
\& \text { title }\end{array}$ & $\begin{array}{l}\text { Berry, Cullen, \& } \\
\text { Meyer (2014) }\end{array}$ & $\begin{array}{l}\text { Study } 2 \text { of } 1 \\
\text { and of previous } \\
\text { research }\end{array}$ & $\begin{array}{l}\text { replication or } \\
\text { replicate }\end{array}$ & dependent & $\begin{array}{l}\text { dependent confounded } \\
\text { replication }\end{array}$ \\
\hline 135 & JAP & $\begin{array}{l}\text { abstract } \\
\& \text { title }\end{array}$ & $\begin{array}{l}\text { Berry, Cullen, \& } \\
\text { Meyer (2014) }\end{array}$ & $\begin{array}{l}\text { Study } 1 \text { of } \\
\text { previous } \\
\text { research }\end{array}$ & $\begin{array}{l}\text { replication or } \\
\text { replicate }\end{array}$ & independent & $\begin{array}{l}\text { independent incremental } \\
\text { constructive replication }\end{array}$ \\
\hline 136 & JAP & full text & $\begin{array}{l}\text { Bezrukova, Spell, } \\
\text { Caldwell, \& Burger } \\
\text { (2016) }\end{array}$ & study 1 & $\begin{array}{l}\text { replication or } \\
\text { replicate }\end{array}$ & independent & $\begin{array}{l}\text { independent quasi-random } \\
\text { replication }\end{array}$ \\
\hline 137 & JAP & full text & $\begin{array}{l}\text { Bindl, Parker, } \\
\text { Totterdell, \& } \\
\text { Hagger-Johnson } \\
(2012)\end{array}$ & $\begin{array}{l}\text { study } 2 \text { of study } \\
1\end{array}$ & $\begin{array}{l}\text { replication or } \\
\text { replicate }\end{array}$ & dependent & $\begin{array}{l}\text { dependent confounded } \\
\text { replication }\end{array}$ \\
\hline 138 & JAP & full text & $\begin{array}{l}\text { Biron \& Bamberger } \\
(2012)\end{array}$ & $\begin{array}{l}\text { study } 1 \text { of } \\
\text { previous } \\
\text { research }\end{array}$ & $\begin{array}{l}\text { replication or } \\
\text { replicate }\end{array}$ & independent & $\begin{array}{l}\text { independent quasi-random } \\
\text { replication }\end{array}$ \\
\hline 139 & JAP & full text & $\begin{array}{l}\text { Blanton, Jaccard, } \\
\text { Strauts, Mitchell, \& } \\
\text { Tetlock (2015) }\end{array}$ & $\begin{array}{l}\text { Study } 1 \text { of } \\
\text { previous } \\
\text { research }\end{array}$ & $\begin{array}{l}\text { replication or } \\
\text { replicate }\end{array}$ & independent & $\begin{array}{l}\text { independent constructive } \\
\text { reproducibility }\end{array}$ \\
\hline
\end{tabular}




\begin{tabular}{|c|c|c|c|c|c|c|c|}
\hline$\#$ & Journal & Search & Study & $\begin{array}{l}\text { Which } \\
\text { repetition }\end{array}$ & Terminology & Independence & $\begin{array}{l}\text { Type of replication or } \\
\text { reproducibility study }\end{array}$ \\
\hline 140 & JAP & full text & $\begin{array}{l}\text { Blanton, Jaccard, } \\
\text { Strauts, Mitchell, \& } \\
\text { Tetlock (2015) }\end{array}$ & $\begin{array}{l}\text { Study } 2 \text { of } \\
\text { previous } \\
\text { research }\end{array}$ & $\begin{array}{l}\text { replication or } \\
\text { replicate }\end{array}$ & independent & $\begin{array}{l}\text { independent constructive } \\
\text { reproducibility }\end{array}$ \\
\hline 141 & JAP & full text & $\begin{array}{l}\text { Bonner, } \\
\text { Greenbaum, \& } \\
\text { Quade }(2017)\end{array}$ & $\begin{array}{l}\text { study } 4 \text { of } \\
\text { studies } 1-3\end{array}$ & $\begin{array}{l}\text { replication or } \\
\text { replicate }\end{array}$ & dependent & $\begin{array}{l}\text { confounded dependent } \\
\text { replication }\end{array}$ \\
\hline 142 & JAP & full text & $\begin{array}{l}\text { Bonner, } \\
\text { Greenbaum, \& } \\
\text { Quade (2017) }\end{array}$ & study 2 of study & nothing & dependent & $\begin{array}{l}\text { confounded dependent } \\
\text { replication }\end{array}$ \\
\hline 143 & JAP & full text & $\begin{array}{l}\text { Bonner, } \\
\text { Greenbaum, \& } \\
\text { Quade (2017) }\end{array}$ & $\begin{array}{l}\text { study } 3 \text { of } \\
\text { studies } 1 \text { and } 2\end{array}$ & nothing & dependent & $\begin{array}{l}\text { dependent substantial } \\
\text { constructive replication }\end{array}$ \\
\hline 144 & JAP & full text & $\begin{array}{l}\text { Brooks, Dalal, \& } \\
\text { Nolan (2014) }\end{array}$ & $\begin{array}{l}\text { study } 2 \text { of study } \\
1\end{array}$ & $\begin{array}{l}\text { replication or } \\
\text { replicate }\end{array}$ & dependent & $\begin{array}{l}\text { dependent quasi-random } \\
\text { replication }\end{array}$ \\
\hline 145 & JAP & full text & $\begin{array}{l}\text { Brosi, Sporrle, } \\
\text { Welpe, \& Heilman } \\
\text { (2016) }\end{array}$ & $\begin{array}{l}\text { study } 2 \text { of study } \\
1\end{array}$ & $\begin{array}{l}\text { replication or } \\
\text { replicate }\end{array}$ & dependent & dependent literal replication \\
\hline 146 & JAP & full text & $\begin{array}{l}\text { Byrne, Peters, \& } \\
\text { Weston (2016) }\end{array}$ & study 1 & $\begin{array}{l}\text { replication or } \\
\text { replicate }\end{array}$ & independent & $\begin{array}{l}\text { independent quasi-random } \\
\text { replication }\end{array}$ \\
\hline 147 & JAP & full text & Caleo (2016) & $\begin{array}{l}\text { study } 2 \text { of study } \\
1\end{array}$ & $\begin{array}{l}\text { replication or } \\
\text { replicate }\end{array}$ & dependent & dependent literal replication \\
\hline
\end{tabular}




\begin{tabular}{|c|c|c|c|c|c|c|c|}
\hline \# & Journal & Search & Study & $\begin{array}{l}\text { Which } \\
\text { repetition }\end{array}$ & Terminology & Independence & $\begin{array}{l}\text { Type of replication or } \\
\text { reproducibility study }\end{array}$ \\
\hline 148 & JAP & full text & $\begin{array}{l}\text { Campagna, Mislin, } \\
\text { Kong, \& Bottom } \\
(2016)\end{array}$ & $\begin{array}{l}\text { study } 3 \text { or study } \\
1 \text { and } 2\end{array}$ & nothing & dependent & $\begin{array}{l}\text { dependent quasi-random } \\
\text { replication }\end{array}$ \\
\hline 149 & JAP & full text & $\begin{array}{l}\text { Campagna, Mislin, } \\
\text { Kong, \& Bottom } \\
(2016)\end{array}$ & $\begin{array}{l}\text { study } 4 \text { of } \\
\text { studies } 1,2,3\end{array}$ & nothing & dependent & $\begin{array}{l}\text { dependent incremental } \\
\text { constructive replication }\end{array}$ \\
\hline 150 & JAP & full text & $\begin{array}{l}\text { Campagna, Mislin, } \\
\text { Kong, \& Bottom } \\
(2016)\end{array}$ & $\begin{array}{l}\text { study } 2 \text { of study } \\
1\end{array}$ & $\begin{array}{l}\text { replication or } \\
\text { replicate }\end{array}$ & dependent & $\begin{array}{l}\text { dependent confounded } \\
\text { replication }\end{array}$ \\
\hline 151 & JAP & $\begin{array}{l}\text { abstract } \\
\& \text { title }\end{array}$ & $\begin{array}{l}\text { Campbell, Liao, } \\
\text { Chuang, Zhou, \& } \\
\text { Dong (2017) }\end{array}$ & $\begin{array}{l}\text { study } 2 \text { of study } \\
1\end{array}$ & $\begin{array}{l}\text { constructive } \\
\text { replication }\end{array}$ & dependent & $\begin{array}{l}\text { dependent confounded } \\
\text { replication }\end{array}$ \\
\hline 152 & JAP & full text & $\begin{array}{l}\text { Carter, Dalal, } \\
\text { Boyce, O'Connell, } \\
\text { Kung, \& Delgado } \\
\text { (2014) }\end{array}$ & $\begin{array}{l}\text { study } 2 \text { of study } \\
1\end{array}$ & $\begin{array}{l}\text { replication or } \\
\text { replicate }\end{array}$ & dependent & $\begin{array}{l}\text { dependent quasi-random } \\
\text { replication }\end{array}$ \\
\hline 153 & JAP & $\begin{array}{l}\text { abstract } \\
\& \text { title }\end{array}$ & $\begin{array}{l}\text { Chen, Chen, \& } \\
\text { Sheldon (2016) }\end{array}$ & $\begin{array}{l}\text { Study } 3 \text { of } \\
\text { Studies } 1 \text { and } 2\end{array}$ & $\begin{array}{l}\text { replication or } \\
\text { replicate }\end{array}$ & dependent & $\begin{array}{l}\text { dependent confounded } \\
\text { replication }\end{array}$ \\
\hline 154 & JAP & $\begin{array}{l}\text { abstract } \\
\& \text { title }\end{array}$ & $\begin{array}{l}\text { Chen, Chen, \& } \\
\text { Sheldon (2016) }\end{array}$ & $\begin{array}{l}\text { Study } 2 \text { of } \\
\text { Study } 1\end{array}$ & $\begin{array}{l}\text { replication or } \\
\text { replicate }\end{array}$ & dependent & $\begin{array}{l}\text { dependent confounded } \\
\text { replication }\end{array}$ \\
\hline
\end{tabular}




\begin{tabular}{|c|c|c|c|c|c|c|c|}
\hline$\#$ & Journal & Search & Study & $\begin{array}{l}\text { Which } \\
\text { repetition }\end{array}$ & Terminology & Independence & $\begin{array}{l}\text { Type of replication or } \\
\text { reproducibility study }\end{array}$ \\
\hline 155 & JAP & full text & $\begin{array}{l}\text { Chen, Myers, } \\
\text { Kopelman, \& } \\
\text { Garcia (2012) }\end{array}$ & study 2 & nothing & dependent & $\begin{array}{l}\text { dependent incremental } \\
\text { constructive replication }\end{array}$ \\
\hline 156 & JAP & full text & $\begin{array}{l}\text { Chen, Myers, } \\
\text { Kopelman, \& } \\
\text { Garcia }(2012)\end{array}$ & $\begin{array}{l}\text { study } 3 \text { of study } \\
1 \text { and } 2\end{array}$ & $\begin{array}{l}\text { replication or } \\
\text { replicate }\end{array}$ & dependent & $\begin{array}{l}\text { dependent quasi-random } \\
\text { replication }\end{array}$ \\
\hline 157 & JAP & full text & $\begin{array}{l}\text { Chi, Li, Wang, \& } \\
\text { Song (2016) }\end{array}$ & & $\begin{array}{l}\text { replication or } \\
\text { replicate }\end{array}$ & dependent & $\begin{array}{l}\text { dependent quasi-random } \\
\text { reproducibility }\end{array}$ \\
\hline 158 & JAP & full text & $\begin{array}{l}\text { da Motta Veiga \& } \\
\text { Gabriel (2016) }\end{array}$ & & $\begin{array}{l}\text { replication or } \\
\text { replicate }\end{array}$ & dependent & $\begin{array}{l}\text { dependent literal } \\
\text { reproducibility }\end{array}$ \\
\hline 159 & JAP & full text & $\begin{array}{l}\text { Dang, Umphress, \& } \\
\text { Mitchell (2017) }\end{array}$ & $\begin{array}{l}\text { study } 2 \text { of study } \\
1\end{array}$ & $\begin{array}{l}\text { replication or } \\
\text { replicate }\end{array}$ & dependent & $\begin{array}{l}\text { dependent confounded } \\
\text { replication }\end{array}$ \\
\hline 160 & JAP & full text & $\begin{array}{l}\text { de Wit, Greer, \& } \\
\text { Jehn (2012) }\end{array}$ & $\begin{array}{l}\text { previous } \\
\text { research }\end{array}$ & $\begin{array}{l}\text { replication or } \\
\text { replicate }\end{array}$ & independent & $\begin{array}{l}\text { independent quasi-random } \\
\text { replication }\end{array}$ \\
\hline 161 & JAP & full text & $\begin{array}{l}\text { DeCelles, DeRue, } \\
\text { Margolis, \& } \\
\text { Ceranic (2012) }\end{array}$ & $\begin{array}{l}\text { study } 2 \text { of study } \\
1\end{array}$ & $\begin{array}{l}\text { replication or } \\
\text { replicate }\end{array}$ & dependent & $\begin{array}{l}\text { dependent quasi-random } \\
\text { replication }\end{array}$ \\
\hline 162 & JAP & full text & $\begin{array}{l}\text { Den Hartog \& } \\
\text { Belschak (2012) }\end{array}$ & $\begin{array}{l}\text { study } 2 \text { of study } \\
1\end{array}$ & $\begin{array}{l}\text { replication or } \\
\text { replicate }\end{array}$ & dependent & $\begin{array}{l}\text { dependent quasi-random } \\
\text { replication }\end{array}$ \\
\hline
\end{tabular}




\begin{tabular}{|c|c|c|c|c|c|c|c|}
\hline \# & Journal & Search & Study & $\begin{array}{l}\text { Which } \\
\text { repetition }\end{array}$ & Terminology & Independence & $\begin{array}{l}\text { Type of replication or } \\
\text { reproducibility study }\end{array}$ \\
\hline 163 & JAP & full text & $\begin{array}{l}\text { Diestel, Rivkin, \& } \\
\text { Schmidt (2015) }\end{array}$ & $\begin{array}{l}\text { study } 2 \text { of } \\
\text { previous } \\
\text { research }\end{array}$ & $\begin{array}{l}\text { replication or } \\
\text { replicate }\end{array}$ & independent & $\begin{array}{l}\text { independent quasi-random } \\
\text { replication }\end{array}$ \\
\hline 164 & JAP & full text & $\begin{array}{l}\text { Diestel, Rivkin, \& } \\
\text { Schmidt (2015) }\end{array}$ & $\begin{array}{l}\text { study } 2 \text { of study } \\
1\end{array}$ & $\begin{array}{l}\text { replication or } \\
\text { replicate }\end{array}$ & dependent & $\begin{array}{l}\text { dependent quasi-random } \\
\text { replication }\end{array}$ \\
\hline 165 & JAP & $\begin{array}{l}\text { abstract } \\
\& \text { title }\end{array}$ & $\begin{array}{l}\text { Dietz, van } \\
\text { Knippenberg, Hirst, } \\
\text { \& Restubog (2015) }\end{array}$ & Study 2 of 1 & $\begin{array}{l}\text { replication or } \\
\text { replicate }\end{array}$ & dependent & $\begin{array}{l}\text { dependent confounded } \\
\text { replication }\end{array}$ \\
\hline 166 & JAP & $\begin{array}{l}\text { abstract } \\
\& \text { title }\end{array}$ & $\begin{array}{l}\text { Dimotakis, Conlon, } \\
\& \text { Ilies (2012) }\end{array}$ & Study 2 of 1 & $\begin{array}{l}\text { replication or } \\
\text { replicate }\end{array}$ & dependent & $\begin{array}{l}\text { dependent incremental } \\
\text { constructive replication }\end{array}$ \\
\hline 167 & JAP & full text & $\begin{array}{l}\text { Dragoni \& Kuenzi } \\
(2012)\end{array}$ & & replicable & dependent & generalizability \\
\hline 168 & JAP & full text & $\begin{array}{l}\text { Dragoni, Park, } \\
\text { Soltis, \& Forte- } \\
\text { Trammell (2014) }\end{array}$ & & $\begin{array}{l}\text { replication or } \\
\text { replicate }\end{array}$ & dependent & $\begin{array}{l}\text { dependent quasi-random } \\
\text { reproducibility }\end{array}$ \\
\hline 169 & JAP & full text & $\begin{array}{l}\text { Duguid \& Thomas- } \\
\text { Hunt (2015) }\end{array}$ & study $1 \mathrm{c}$ & nothing & dependent & $\begin{array}{l}\text { dependent quasi-random } \\
\text { replication }\end{array}$ \\
\hline 170 & JAP & full text & $\begin{array}{l}\text { Duguid \& Thomas- } \\
\text { Hunt (2015) }\end{array}$ & study 3 & nothing & dependent & generalizability \\
\hline 171 & JAP & full text & $\begin{array}{l}\text { Duguid \& Thomas- } \\
\text { Hunt (2015) }\end{array}$ & study 4 & nothing & dependent & generalizability \\
\hline
\end{tabular}




\begin{tabular}{|c|c|c|c|c|c|c|c|}
\hline$\#$ & Journal & Search & Study & $\begin{array}{l}\text { Which } \\
\text { repetition }\end{array}$ & Terminology & Independence & $\begin{array}{l}\text { Type of replication or } \\
\text { reproducibility study }\end{array}$ \\
\hline 172 & JAP & full text & $\begin{array}{l}\text { Duguid \& Thomas- } \\
\text { Hunt (2015) }\end{array}$ & study $1 \mathrm{~b}$ of $1 \mathrm{a}$ & $\begin{array}{l}\text { replication or } \\
\text { replicate }\end{array}$ & dependent & $\begin{array}{l}\text { dependent quasi-random } \\
\text { replication }\end{array}$ \\
\hline 173 & JAP & full text & $\begin{array}{l}\text { Duguid \& Thomas- } \\
\text { Hunt (2015) }\end{array}$ & $\begin{array}{l}\text { study } 2 \text { of study } \\
\text { 1a }\end{array}$ & $\begin{array}{l}\text { replication or } \\
\text { replicate }\end{array}$ & dependent & $\begin{array}{l}\text { dependent incremental } \\
\text { constructive replication }\end{array}$ \\
\hline 174 & JAP & full text & $\begin{array}{l}\text { Eberly,Holley, } \\
\text { Johnson, \& } \\
\text { Mitchell (2017) }\end{array}$ & study $1 \mathrm{~d}$ of $1 \mathrm{c}$ & $\begin{array}{l}\text { replication or } \\
\text { replicate }\end{array}$ & dependent & $\begin{array}{l}\text { dependent quasi-random } \\
\text { replication }\end{array}$ \\
\hline 175 & JAP & full text & $\begin{array}{l}\text { Eberly,Holley, } \\
\text { Johnson, \& } \\
\text { Mitchell (2017) }\end{array}$ & study 1 e of $1 d$ & $\begin{array}{l}\text { replication or } \\
\text { replicate }\end{array}$ & dependent & $\begin{array}{l}\text { dependent quasi-random } \\
\text { replication }\end{array}$ \\
\hline 176 & JAP & full text & $\begin{array}{l}\text { Eberly,Holley, } \\
\text { Johnson, \& } \\
\text { Mitchell (2017) }\end{array}$ & $\begin{array}{l}\text { Study } 3 \text { of } \\
\text { Study } 2\end{array}$ & $\begin{array}{l}\text { replication or } \\
\text { replicate }\end{array}$ & dependent & $\begin{array}{l}\text { dependent quasi-random } \\
\text { replication }\end{array}$ \\
\hline 177 & JAP & $\begin{array}{l}\text { abstract } \\
\& \text { title }\end{array}$ & $\begin{array}{l}\text { Eby, Durley, Evans, } \\
\text { \& Ragins } 2008\end{array}$ & Study 3 of 2 & $\begin{array}{l}\text { replication or } \\
\text { replicate }\end{array}$ & dependent & $\begin{array}{l}\text { dependent incremental } \\
\text { constructive replication }\end{array}$ \\
\hline 178 & JAP & full text & $\begin{array}{l}\text { Ellingson, Tews, \& } \\
\text { Dachner (2016) }\end{array}$ & $\begin{array}{l}\text { sample } 2 \text { of } \\
\text { sample } 1\end{array}$ & $\begin{array}{l}\text { constructive } \\
\text { replication }\end{array}$ & dependent & $\begin{array}{l}\text { dependent quasi-random } \\
\text { replication }\end{array}$ \\
\hline 179 & JAP & full text & $\begin{array}{l}\text { Ellington, } \\
\text { Dierdorff, \& Rubin } \\
\text { (2014) }\end{array}$ & $\begin{array}{l}\text { previous } \\
\text { research }\end{array}$ & $\begin{array}{l}\text { replication or } \\
\text { replicate }\end{array}$ & independent & $\begin{array}{l}\text { independent quasi-random } \\
\text { replication }\end{array}$ \\
\hline
\end{tabular}




\begin{tabular}{|c|c|c|c|c|c|c|c|}
\hline$\#$ & Journal & Search & Study & $\begin{array}{l}\text { Which } \\
\text { repetition }\end{array}$ & Terminology & Independence & $\begin{array}{l}\text { Type of replication or } \\
\text { reproducibility study }\end{array}$ \\
\hline 180 & JAP & full text & $\begin{array}{l}\text { Ellis, Mai, \& } \\
\text { Christian (2013) }\end{array}$ & $\begin{array}{l}\text { previous } \\
\text { research }\end{array}$ & $\begin{array}{l}\text { replication or } \\
\text { replicate }\end{array}$ & independent & $\begin{array}{l}\text { independent quasi-random } \\
\text { replication }\end{array}$ \\
\hline 181 & JAP & full text & $\begin{array}{l}\text { Farh \& Chen } \\
\text { (2014) }\end{array}$ & $\begin{array}{l}\text { study } 2 \text { of study } \\
1\end{array}$ & $\begin{array}{l}\text { constructive } \\
\text { replication }\end{array}$ & dependent & $\begin{array}{l}\text { dependent confounded } \\
\text { replication }\end{array}$ \\
\hline 182 & JAP & full text & $\begin{array}{l}\text { Farh, Seo, \& Tesluk } \\
\text { (2012) }\end{array}$ & $\begin{array}{l}\text { previous } \\
\text { research }\end{array}$ & $\begin{array}{l}\text { replication or } \\
\text { replicate }\end{array}$ & independent & $\begin{array}{l}\text { independent quasi-random } \\
\text { replication }\end{array}$ \\
\hline 183 & JAP & full text & $\begin{array}{l}\text { Ferguson \& } \\
\text { Peterson (2015) }\end{array}$ & $\begin{array}{l}\text { previous } \\
\text { research }\end{array}$ & $\begin{array}{l}\text { replication or } \\
\text { replicate }\end{array}$ & independent & $\begin{array}{l}\text { independent quasi-random } \\
\text { replication }\end{array}$ \\
\hline 184 & JAP & $\begin{array}{l}\text { abstract } \\
\& \text { title }\end{array}$ & $\begin{array}{l}\text { Ferris, Brown, } \\
\text { Berry, \& Lian } \\
(2008)\end{array}$ & Study 2 of 1 & $\begin{array}{l}\text { replication or } \\
\text { replicate }\end{array}$ & dependent & dependent literal replication \\
\hline 185 & JAP & $\begin{array}{l}\text { abstract } \\
\& \text { title }\end{array}$ & $\begin{array}{l}\text { Ferris, Brown, } \\
\text { Berry, \& Lian } \\
(2008)\end{array}$ & Study 3 of 2 & $\begin{array}{l}\text { replication or } \\
\text { replicate }\end{array}$ & dependent & dependent literal replication \\
\hline 186 & JAP & $\begin{array}{l}\text { abstract } \\
\& \text { title }\end{array}$ & $\begin{array}{l}\text { Ferris, Brown, } \\
\text { Berry, \& Lian } \\
(2008)\end{array}$ & Study 4 of 3 & $\begin{array}{l}\text { replication or } \\
\text { replicate }\end{array}$ & dependent & dependent literal replication \\
\hline 187 & JAP & full text & $\begin{array}{l}\text { Ferris, Johnson, } \\
\text { Rosen, Djurdjevic, } \\
\text { Chang, \& Tan } \\
\text { (2013) }\end{array}$ & $\begin{array}{l}\text { sample } 2 \text { of } \\
\text { sample } 1\end{array}$ & $\begin{array}{l}\text { replication or } \\
\text { replicate }\end{array}$ & dependent & $\begin{array}{l}\text { dependent confounded } \\
\text { replication }\end{array}$ \\
\hline
\end{tabular}




\begin{tabular}{|c|c|c|c|c|c|c|c|}
\hline \# & Journal & Search & Study & $\begin{array}{l}\text { Which } \\
\text { repetition }\end{array}$ & Terminology & Independence & $\begin{array}{l}\text { Type of replication or } \\
\text { reproducibility study }\end{array}$ \\
\hline 188 & JAP & full text & $\begin{array}{l}\text { Fine, Goldenberg, } \\
\& \text { Noam (2016) }\end{array}$ & & $\begin{array}{l}\text { replication or } \\
\text { replicate }\end{array}$ & dependent & $\begin{array}{l}\text { dependent quasi-random } \\
\text { reproducibility }\end{array}$ \\
\hline 189 & JAP & full text & Fisher (2014) & $\begin{array}{l}\text { previous } \\
\text { research }\end{array}$ & $\begin{array}{l}\text { conceptual } \\
\text { replication }\end{array}$ & independent & $\begin{array}{l}\text { independent quasi-random } \\
\text { replication }\end{array}$ \\
\hline 190 & JAP & full text & Fisher (2014) & & $\begin{array}{l}\text { replication or } \\
\text { replicate }\end{array}$ & dependent & generalizability \\
\hline 191 & JAP & full text & $\begin{array}{l}\text { Fisher, Bell, } \\
\text { Dierdorff, \& } \\
\text { Belohlav (2012) }\end{array}$ & $\begin{array}{l}\text { previous } \\
\text { research }\end{array}$ & $\begin{array}{l}\text { substantive } \\
\text { replication }\end{array}$ & independent & $\begin{array}{l}\text { independent quasi-random } \\
\text { replication }\end{array}$ \\
\hline 192 & JAP & full text & $\begin{array}{l}\text { Foulk \& Long } \\
\text { (2016) }\end{array}$ & study $1 \mathrm{c}$ & nothing & dependent & generalizability \\
\hline 193 & JAP & full text & $\begin{array}{l}\text { Foulk \& Long } \\
(2016)\end{array}$ & study $1 \mathrm{~d}$ & nothing & dependent & generalizability \\
\hline 194 & JAP & full text & $\begin{array}{l}\text { Foulk \& Long } \\
\text { (2016) }\end{array}$ & study $1 \mathrm{e}$ & nothing & dependent & generalizability \\
\hline 195 & JAP & full text & $\begin{array}{l}\text { Foulk \& Long } \\
\text { (2016) }\end{array}$ & study2 & nothing & dependent & generalizability \\
\hline 196 & JAP & full text & $\begin{array}{l}\text { Foulk \& Long } \\
(2016)\end{array}$ & $\begin{array}{l}\text { study } 1 \mathrm{~b} \text { of } \\
\text { study } 1 \mathrm{a}\end{array}$ & $\begin{array}{l}\text { replication or } \\
\text { replicate }\end{array}$ & dependent & $\begin{array}{l}\text { dependent quasi-random } \\
\text { replication }\end{array}$ \\
\hline
\end{tabular}




\begin{tabular}{|c|c|c|c|c|c|c|c|}
\hline \# & Journal & Search & Study & $\begin{array}{l}\text { Which } \\
\text { repetition }\end{array}$ & Terminology & Independence & $\begin{array}{l}\text { Type of replication or } \\
\text { reproducibility study }\end{array}$ \\
\hline 197 & JAP & full text & $\begin{array}{l}\text { Gabriel, Daniels, } \\
\text { Diefendorff, \& } \\
\text { Greguras (2015) }\end{array}$ & $\begin{array}{l}3 \text { subsamples in } \\
\text { overall sample } \\
\text { of study } 1\end{array}$ & $\begin{array}{l}\text { replication or } \\
\text { replicate }\end{array}$ & dependent & dependent literal replication \\
\hline 198 & JAP & full text & $\begin{array}{l}\text { Gabriel, Daniels, } \\
\text { Diefendorff, \& } \\
\text { Greguras (2015) }\end{array}$ & $\begin{array}{l}\text { study } 2 \text { of study } \\
1\end{array}$ & $\begin{array}{l}\text { replication or } \\
\text { replicate }\end{array}$ & dependent & $\begin{array}{l}\text { dependent quasi-random } \\
\text { replication }\end{array}$ \\
\hline 199 & JAP & $\begin{array}{l}\text { abstract } \\
\& \text { title }\end{array}$ & $\begin{array}{l}\text { Garcia, Restubog, } \\
\text { Kiewitz, Scott, \& } \\
\text { Tang (2014) }\end{array}$ & Study 2 of 1 & $\begin{array}{l}\text { constructive } \\
\text { replication }\end{array}$ & dependent & $\begin{array}{l}\text { dependent incremental } \\
\text { constructive replication }\end{array}$ \\
\hline 200 & JAP & $\begin{array}{l}\text { abstract } \\
\& \text { title }\end{array}$ & $\begin{array}{l}\text { Garcia, Restubog, } \\
\text { Kiewitz, Scott, \& } \\
\text { Tang (2014) }\end{array}$ & $\begin{array}{l}\text { Study } 3 \text { of } 1 \\
\text { and } 2\end{array}$ & $\begin{array}{l}\text { constructive } \\
\text { replication }\end{array}$ & dependent & $\begin{array}{l}\text { dependent comprehensive } \\
\text { constructive replication }\end{array}$ \\
\hline 201 & JAP & $\begin{array}{l}\text { abstract } \\
\& \text { title }\end{array}$ & $\begin{array}{l}\text { Garcia, Restubog, } \\
\text { Kiewitz, Scott, \& } \\
\text { Tang (2014) }\end{array}$ & Study 4 of $1-3$ & $\begin{array}{l}\text { constructive } \\
\text { replication }\end{array}$ & dependent & $\begin{array}{l}\text { dependent confounded } \\
\text { replication }\end{array}$ \\
\hline 202 & JAP & full text & $\begin{array}{l}\text { Gelfand, Brett, } \\
\text { Gunia, Imai, } \\
\text { Huang, \& Hsu } \\
\text { (2013) }\end{array}$ & $\begin{array}{l}\text { experiment } 2 \text { of } \\
\text { experiment } 1\end{array}$ & $\begin{array}{l}\text { replication or } \\
\text { replicate }\end{array}$ & dependent & $\begin{array}{l}\text { dependent quasi-random } \\
\text { replication }\end{array}$ \\
\hline 203 & JAP & full text & $\begin{array}{l}\text { Gerbasi, Porath, } \\
\text { Parker, Spreitzer, \& } \\
\text { Cross }(2015)\end{array}$ & $\begin{array}{l}\text { study } 2 \text { of study } \\
1\end{array}$ & $\begin{array}{l}\text { replication or } \\
\text { replicate }\end{array}$ & dependent & $\begin{array}{l}\text { dependent quasi-random } \\
\text { replication }\end{array}$ \\
\hline
\end{tabular}




\begin{tabular}{|c|c|c|c|c|c|c|c|}
\hline$\#$ & Journal & Search & Study & $\begin{array}{l}\text { Which } \\
\text { repetition }\end{array}$ & Terminology & Independence & $\begin{array}{l}\text { Type of replication or } \\
\text { reproducibility study }\end{array}$ \\
\hline 204 & JAP & full text & $\begin{array}{l}\text { Giessner, van } \\
\text { Knippenberg, van } \\
\text { Ginkel, \& Sleebos } \\
\text { (2013) }\end{array}$ & $\begin{array}{l}\text { study } 2 \text { of study } \\
1\end{array}$ & $\begin{array}{l}\text { replication or } \\
\text { replicate }\end{array}$ & dependent & $\begin{array}{l}\text { dependent confounded } \\
\text { replication }\end{array}$ \\
\hline 205 & JAP & full text & $\begin{array}{l}\text { Giumetti, } \\
\text { Schroeder, \& } \\
\text { Switzer (2015) }\end{array}$ & $\begin{array}{l}\text { previous } \\
\text { research }\end{array}$ & $\begin{array}{l}\text { replication or } \\
\text { replicate }\end{array}$ & independent & $\begin{array}{l}\text { independent quasi-random } \\
\text { replication }\end{array}$ \\
\hline 206 & JAP & full text & $\begin{array}{l}\text { Grand, Braun, } \\
\text { Kuljanin, } \\
\text { Kozlowski, \& Chao } \\
\text { (2016) }\end{array}$ & $\begin{array}{l}\text { step 4: actual } \\
\text { virtual teams }\end{array}$ & $\begin{array}{l}\text { replication or } \\
\text { replicate }\end{array}$ & dependent & $\begin{array}{l}\text { dependent incremental } \\
\text { constructive replication }\end{array}$ \\
\hline 207 & JAP & $\begin{array}{l}\text { abstract } \\
\& \text { title }\end{array}$ & $\begin{array}{l}\text { Grant \& Rothbard } \\
2013\end{array}$ & Study 2 of 1 & $\begin{array}{l}\text { replication or } \\
\text { replicate }\end{array}$ & dependent & $\begin{array}{l}\text { dependent confounded } \\
\text { replication }\end{array}$ \\
\hline 208 & JAP & full text & $\begin{array}{l}\text { Greenbaum, Quade, } \\
\text { Mawritz, Kim, \& } \\
\text { Crosby (2014) }\end{array}$ & $\begin{array}{l}\text { study } 2 \text { of study } \\
1\end{array}$ & $\begin{array}{l}\text { replication or } \\
\text { replicate }\end{array}$ & dependent & $\begin{array}{l}\text { dependent substantial } \\
\text { constructive replication }\end{array}$ \\
\hline 209 & JAP & full text & $\begin{array}{l}\text { Guarana \& } \\
\text { Hernandez (2016) }\end{array}$ & $\begin{array}{l}\text { study } 3 \text { of study } \\
2 \text { and } 1\end{array}$ & nothing & dependent & $\begin{array}{l}\text { dependent quasi-random } \\
\text { replication }\end{array}$ \\
\hline 210 & JAP & full text & $\begin{array}{l}\text { Guarana \& } \\
\text { Hernandez (2016) }\end{array}$ & $\begin{array}{l}\text { study } 2 \text { of study } \\
1\end{array}$ & nothing & dependent & $\begin{array}{l}\text { dependent incremental } \\
\text { constructive replication }\end{array}$ \\
\hline
\end{tabular}




\begin{tabular}{|c|c|c|c|c|c|c|c|}
\hline \# & Journal & Search & Study & $\begin{array}{l}\text { Which } \\
\text { repetition }\end{array}$ & Terminology & Independence & $\begin{array}{l}\text { Type of replication or } \\
\text { reproducibility study }\end{array}$ \\
\hline 211 & JAP & full text & $\begin{array}{l}\text { Guarana \& } \\
\text { Hernandez (2016) }\end{array}$ & $\begin{array}{l}\text { study } 4 \text { of study } \\
1,2, \text { and } 3\end{array}$ & $\begin{array}{l}\text { replication or } \\
\text { replicate }\end{array}$ & dependent & $\begin{array}{l}\text { dependent quasi-random } \\
\text { replication }\end{array}$ \\
\hline 212 & JAP & full text & $\begin{array}{l}\text { Guarana \& } \\
\text { Hernandez (2016) }\end{array}$ & $\begin{array}{l}\text { study } 1 \text { of } \\
\text { previous } \\
\text { research }\end{array}$ & $\begin{array}{l}\text { replication or } \\
\text { replicate }\end{array}$ & independent & $\begin{array}{l}\text { independent quasi-random } \\
\text { replication }\end{array}$ \\
\hline 213 & JAP & $\begin{array}{l}\text { abstract } \\
\& \text { title }\end{array}$ & $\begin{array}{l}\text { Gunia, Brett, } \\
\text { Nandkeolyar, \& } \\
\text { Kamdar (2011) }\end{array}$ & $\begin{array}{l}\text { study } 3 \text { of study } \\
2\end{array}$ & $\begin{array}{l}\text { replicate and } \\
\text { extend }\end{array}$ & dependent & $\begin{array}{l}\text { dependent substantial } \\
\text { constructive replication }\end{array}$ \\
\hline 214 & JAP & $\begin{array}{l}\text { abstract } \\
\& \text { title }\end{array}$ & $\begin{array}{l}\text { Gunia, Brett, } \\
\text { Nandkeolyar, \& } \\
\text { Kamdar (2011) }\end{array}$ & $\begin{array}{l}\text { study } 2 \text { of study } \\
1\end{array}$ & nothing & dependent & $\begin{array}{l}\text { incremental dependent } \\
\text { replication }\end{array}$ \\
\hline 215 & JAP & full text & $\begin{array}{l}\text { Gupta, Ganster, \& } \\
\text { Kepes (2013) }\end{array}$ & $\begin{array}{l}\text { sample } 2 \text { of } \\
\text { sample } 1\end{array}$ & $\begin{array}{l}\text { replication or } \\
\text { replicate }\end{array}$ & dependent & $\begin{array}{l}\text { dependent incremental } \\
\text { constructive replication }\end{array}$ \\
\hline 216 & JAP & full text & $\begin{array}{l}\text { Hahn \& Dormann } \\
(2013)\end{array}$ & $\begin{array}{l}\text { previous } \\
\text { research }\end{array}$ & $\begin{array}{l}\text { replication or } \\
\text { replicate }\end{array}$ & independent & $\begin{array}{l}\text { independent quasi-random } \\
\text { replication }\end{array}$ \\
\hline 217 & JAP & $\begin{array}{l}\text { abstract } \\
\text { \& title }\end{array}$ & $\begin{array}{l}\text { Hanek, Garcia, \& } \\
\text { Tor }(2016)\end{array}$ & Study $1 \mathrm{~b}$ of $1 \mathrm{a}$ & nothing & dependent & $\begin{array}{l}\text { dependent quasi-random } \\
\text { replication }\end{array}$ \\
\hline 218 & JAP & $\begin{array}{l}\text { abstract } \\
\& \text { title }\end{array}$ & $\begin{array}{l}\text { Hanek, Garcia, \& } \\
\text { Tor }(2016)\end{array}$ & $\begin{array}{l}5 b \text { of } 5 a \text { and of } \\
4\end{array}$ & nothing & dependent & $\begin{array}{l}\text { dependent incremental } \\
\text { constructive replication }\end{array}$ \\
\hline
\end{tabular}




\begin{tabular}{|c|c|c|c|c|c|c|c|}
\hline \# & Journal & Search & Study & $\begin{array}{l}\text { Which } \\
\text { repetition }\end{array}$ & Terminology & Independence & $\begin{array}{l}\text { Type of replication or } \\
\text { reproducibility study }\end{array}$ \\
\hline 219 & JAP & $\begin{array}{l}\text { abstract } \\
\& \text { title }\end{array}$ & $\begin{array}{l}\text { Hanek, Garcia, \& } \\
\text { Tor (2016) }\end{array}$ & $\begin{array}{l}\text { Study } 2 \mathrm{a} \text { of } \\
\text { Studies } 1 \mathrm{a} \text { and } \\
\text { b }\end{array}$ & $\begin{array}{l}\text { replication or } \\
\text { replicate }\end{array}$ & dependent & $\begin{array}{l}\text { dependent confounded } \\
\text { replication }\end{array}$ \\
\hline 220 & JAP & $\begin{array}{l}\text { abstract } \\
\& \text { title }\end{array}$ & $\begin{array}{l}\text { Hanek, Garcia, \& } \\
\text { Tor (2016) }\end{array}$ & Study $5 \mathrm{a}$ of $1-4$ & $\begin{array}{l}\text { replication or } \\
\text { replicate }\end{array}$ & dependent & dependent literal replication \\
\hline 221 & JAP & $\begin{array}{l}\text { abstract } \\
\& \text { title }\end{array}$ & $\begin{array}{l}\text { Hanek, Garcia, \& } \\
\text { Tor (2016) }\end{array}$ & Study 4 of $1-3$ & $\begin{array}{l}\text { replication or } \\
\text { replicate }\end{array}$ & dependent & $\begin{array}{l}\text { dependent quasi-random } \\
\text { replication }\end{array}$ \\
\hline 222 & JAP & $\begin{array}{l}\text { abstract } \\
\& \text { title }\end{array}$ & $\begin{array}{l}\text { Hanek, Garcia, \& } \\
\text { Tor }(2016)\end{array}$ & $\begin{array}{l}\text { study } 2 \mathrm{~b} \text { of } \\
\text { study } 2 \mathrm{a}\end{array}$ & $\begin{array}{l}\text { replication or } \\
\text { replicate }\end{array}$ & dependent & $\begin{array}{l}\text { dependent quasi-random } \\
\text { replication }\end{array}$ \\
\hline 223 & JAP & full text & $\begin{array}{l}\text { Harman, Ellington, } \\
\text { Surface, \& } \\
\text { Thompson (2015) }\end{array}$ & $\begin{array}{l}\text { study } 3 \text { of } \\
\text { studies } 1 \text { and } 2\end{array}$ & $\begin{array}{l}\text { replication or } \\
\text { replicate }\end{array}$ & dependent & $\begin{array}{l}\text { dependent substantial } \\
\text { constructive replication }\end{array}$ \\
\hline 224 & JAP & full text & $\begin{array}{l}\text { Harman, Ellington, } \\
\text { Surface, \& } \\
\text { Thompson (2015) }\end{array}$ & $\begin{array}{l}\text { study } 2 \text { of study } \\
1\end{array}$ & $\begin{array}{l}\text { replication or } \\
\text { replicate }\end{array}$ & dependent & $\begin{array}{l}\text { dependent quasi-random } \\
\text { replication }\end{array}$ \\
\hline 225 & JAP & full text & $\begin{array}{l}\text { Harold, Oh, Holtz, } \\
\text { Han, \& Giacalone } \\
(2016)\end{array}$ & $\begin{array}{l}\text { study } 2 \text { of study } \\
1\end{array}$ & $\begin{array}{l}\text { constructive } \\
\text { replication }\end{array}$ & dependent & $\begin{array}{l}\text { dependent regressive } \\
\text { replication }\end{array}$ \\
\hline 226 & JAP & full text & $\begin{array}{l}\text { Hernandez, Avery, } \\
\text { Tonidandel, Hebl, }\end{array}$ & $\begin{array}{l}\text { study } 3 \text { of } 1 \text { and } \\
2\end{array}$ & triangulate & dependent & $\begin{array}{l}\text { dependent quasi-random } \\
\text { replication }\end{array}$ \\
\hline
\end{tabular}




\begin{tabular}{|c|c|c|c|c|c|c|c|}
\hline$\#$ & Journal & Search & Study & $\begin{array}{l}\text { Which } \\
\text { repetition }\end{array}$ & Terminology & Independence & $\begin{array}{l}\text { Type of replication or } \\
\text { reproducibility study }\end{array}$ \\
\hline & & & $\begin{array}{l}\text { Smith, \& McKay } \\
\text { (2016) }\end{array}$ & & & & \\
\hline 227 & JAP & full text & $\begin{array}{l}\text { Hernandez, Avery, } \\
\text { Tonidandel, Hebl, } \\
\text { Smith, \& McKay } \\
\text { (2016) }\end{array}$ & $\begin{array}{l}\text { study } 4 \text { and } 1, \\
2, \text { and } 3\end{array}$ & $\begin{array}{l}\text { replication or } \\
\text { replicate }\end{array}$ & dependent & $\begin{array}{l}\text { dependent quasi-random } \\
\text { replication }\end{array}$ \\
\hline 228 & JAP & full text & $\begin{array}{l}\text { Hernandez, Avery, } \\
\text { Tonidandel, Hebl, } \\
\text { Smith, \& McKay } \\
\text { (2016) }\end{array}$ & study 2 of 1 & triangulate & dependent & $\begin{array}{l}\text { dependent confounded } \\
\text { replication }\end{array}$ \\
\hline 229 & JAP & $\begin{array}{l}\text { abstract } \\
\& \text { title }\end{array}$ & $\begin{array}{l}\text { Hershcovis \& } \\
\text { Bhatnagar (2017) }\end{array}$ & $\begin{array}{l}\text { study } 2 \text { of study } \\
1\end{array}$ & $\begin{array}{l}\text { replication or } \\
\text { replicate }\end{array}$ & dependent & $\begin{array}{l}\text { dependent confounded } \\
\text { replication }\end{array}$ \\
\hline 230 & JAP & full text & $\begin{array}{l}\text { Hideg \& Ferris } \\
\text { (2017) }\end{array}$ & $\begin{array}{l}\text { supplementary } \\
\text { data collection } \\
\text { of study } 2\end{array}$ & $\begin{array}{l}\text { replication or } \\
\text { replicate }\end{array}$ & dependent & $\begin{array}{l}\text { dependent quasi-random } \\
\text { replication }\end{array}$ \\
\hline 231 & JAP & full text & $\begin{array}{l}\text { Hideg \& Ferris } \\
\text { (2017) }\end{array}$ & $\begin{array}{l}\text { study } 2 \text { of study } \\
1\end{array}$ & $\begin{array}{l}\text { constructive } \\
\text { replication }\end{array}$ & dependent & $\begin{array}{l}\text { dependent quasi-random } \\
\text { replication }\end{array}$ \\
\hline 232 & JAP & full text & $\begin{array}{l}\text { Hideg \& Ferris } \\
(2017)\end{array}$ & $\begin{array}{l}\text { supplementary } \\
\text { data collection } \\
\text { of study } 4\end{array}$ & $\begin{array}{l}\text { replication or } \\
\text { replicate }\end{array}$ & dependent & $\begin{array}{l}\text { dependent quasi-random } \\
\text { replication }\end{array}$ \\
\hline 233 & JAP & full text & $\begin{array}{l}\text { Hideg \& Ferris } \\
(2017)\end{array}$ & $\begin{array}{l}\text { study } 4 \text { of study } \\
3\end{array}$ & $\begin{array}{l}\text { constructive } \\
\text { replication }\end{array}$ & dependent & $\begin{array}{l}\text { dependent quasi-random } \\
\text { replication }\end{array}$ \\
\hline
\end{tabular}




\begin{tabular}{|c|c|c|c|c|c|c|c|}
\hline \# & Journal & Search & Study & $\begin{array}{l}\text { Which } \\
\text { repetition }\end{array}$ & Terminology & Independence & $\begin{array}{l}\text { Type of replication or } \\
\text { reproducibility study }\end{array}$ \\
\hline 234 & JAP & full text & $\begin{array}{l}\text { Hideg \& Ferris } \\
(2017)\end{array}$ & $\begin{array}{l}\text { study } 3 \text { of study } \\
2\end{array}$ & $\begin{array}{l}\text { constructive } \\
\text { replication }\end{array}$ & dependent & $\begin{array}{l}\text { dependent quasi-random } \\
\text { replication }\end{array}$ \\
\hline 235 & JAP & full text & $\begin{array}{l}\text { Hillebrandt \& } \\
\text { Barclay (2017) }\end{array}$ & $\begin{array}{l}\text { study } 3 \text { of study } \\
1 \text { and } 2\end{array}$ & $\begin{array}{l}\text { replication or } \\
\text { replicate }\end{array}$ & dependent & $\begin{array}{l}\text { dependent quasi-random } \\
\text { replication }\end{array}$ \\
\hline 236 & JAP & full text & $\begin{array}{l}\text { Hillebrandt \& } \\
\text { Barclay (2017) }\end{array}$ & $\begin{array}{l}\text { study } 4 \text { or study } \\
1,2, \& 3\end{array}$ & $\begin{array}{l}\text { replication or } \\
\text { replicate }\end{array}$ & dependent & $\begin{array}{l}\text { dependent quasi-random } \\
\text { replication }\end{array}$ \\
\hline 237 & JAP & full text & $\begin{array}{l}\text { Hillebrandt \& } \\
\text { Barclay (2017) }\end{array}$ & $\begin{array}{l}\text { study } 2 \text { of study } \\
1\end{array}$ & $\begin{array}{l}\text { replication or } \\
\text { replicate }\end{array}$ & dependent & $\begin{array}{l}\text { dependent quasi-random } \\
\text { replication }\end{array}$ \\
\hline 238 & JAP & $\begin{array}{l}\text { abstract } \\
\text { \& title }\end{array}$ & $\begin{array}{l}\text { Hochwarter, Ferris, } \\
\text { Zinko, Arnell, \& } \\
\text { James (2007) }\end{array}$ & Study 2 of 1 & $\begin{array}{l}\text { constructive } \\
\text { replication }\end{array}$ & dependent & $\begin{array}{l}\text { dependent substantial } \\
\text { constructive replication }\end{array}$ \\
\hline 239 & JAP & $\begin{array}{l}\text { abstract } \\
\& \text { title }\end{array}$ & $\begin{array}{l}\text { Huang, Hsieh, \& } \\
\text { He (2014) }\end{array}$ & Study 2 of 1 & $\begin{array}{l}\text { replication or } \\
\text { replicate }\end{array}$ & dependent & $\begin{array}{l}\text { dependent confounded } \\
\text { replication }\end{array}$ \\
\hline 240 & JAP & full text & $\begin{array}{l}\text { Huang, Wellman, } \\
\text { Ashford, Lee, \& } \\
\text { Wang (2017) }\end{array}$ & $\begin{array}{l}\text { study } 2 \text { of study } \\
1\end{array}$ & $\begin{array}{l}\text { constructive } \\
\text { replication }\end{array}$ & dependent & $\begin{array}{l}\text { dependent quasi-random } \\
\text { replication }\end{array}$ \\
\hline 241 & JAP & full text & $\begin{array}{l}\text { Huang, Liu, \& } \\
\text { Bowling (2015) }\end{array}$ & $\begin{array}{l}\text { study } 3 \text { of study } \\
1\end{array}$ & $\begin{array}{l}\text { constructive } \\
\text { replication }\end{array}$ & dependent & $\begin{array}{l}\text { dependent quasi-random } \\
\text { replication }\end{array}$ \\
\hline 242 & JAP & full text & Hulsheger (2016) & $\begin{array}{l}\text { previous } \\
\text { research }\end{array}$ & $\begin{array}{l}\text { replication or } \\
\text { replicate }\end{array}$ & independent & $\begin{array}{l}\text { independent quasi-random } \\
\text { replication }\end{array}$ \\
\hline
\end{tabular}




\begin{tabular}{|c|c|c|c|c|c|c|c|}
\hline \# & Journal & Search & Study & $\begin{array}{l}\text { Which } \\
\text { repetition }\end{array}$ & Terminology & Independence & $\begin{array}{l}\text { Type of replication or } \\
\text { reproducibility study }\end{array}$ \\
\hline 243 & JAP & full text & $\begin{array}{l}\text { Hulsheger, Alberts, } \\
\text { Feinholdt, \& Lang } \\
\text { (2013) }\end{array}$ & & $\begin{array}{l}\text { replication or } \\
\text { replicate }\end{array}$ & dependent & $\begin{array}{l}\text { dependent quasi-random } \\
\text { reproducibility }\end{array}$ \\
\hline 244 & JAP & full text & $\begin{array}{l}\text { Hulsheger, Lang, } \\
\text { Schewe, \& Zijlstra } \\
(2015)\end{array}$ & $\begin{array}{l}\text { study } 2 \text { of study } \\
1\end{array}$ & $\begin{array}{l}\text { replication or } \\
\text { replicate }\end{array}$ & dependent & $\begin{array}{l}\text { dependent confounded } \\
\text { replication }\end{array}$ \\
\hline 245 & JAP & full text & $\begin{array}{l}\text { Jiang \& Probst } \\
(2017)\end{array}$ & $\begin{array}{l}\text { study } 2 \text { of study } \\
1\end{array}$ & $\begin{array}{l}\text { constructive } \\
\text { replication }\end{array}$ & dependent & $\begin{array}{l}\text { dependent substantial } \\
\text { constructive replication }\end{array}$ \\
\hline 246 & JAP & full text & $\begin{array}{l}\text { Johnson, Ilies, \& } \\
\text { Boles (2012) }\end{array}$ & study $1 \mathrm{~b}$ of $1 \mathrm{a}$ & nothing & dependent & $\begin{array}{l}\text { dependent incremental } \\
\text { constructive replication }\end{array}$ \\
\hline 247 & JAP & full text & $\begin{array}{l}\text { Johnson, Ilies, \& } \\
\text { Boles (2012) }\end{array}$ & $\begin{array}{l}\text { study } 1 \mathrm{c} \text { of } 1 \mathrm{a} \\
\text { and } 1 \mathrm{~b}\end{array}$ & nothing & dependent & $\begin{array}{l}\text { dependent incremental } \\
\text { constructive replication }\end{array}$ \\
\hline 248 & JAP & full text & $\begin{array}{l}\text { Johnson, Ilies, \& } \\
\text { Boles (2012) }\end{array}$ & study 2 & nothing & dependent & $\begin{array}{l}\text { dependent incremental } \\
\text { constructive replication }\end{array}$ \\
\hline 249 & JAP & full text & $\begin{array}{l}\text { Johnson, Ilies, \& } \\
\text { Boles (2012) }\end{array}$ & $\begin{array}{l}\text { post-hoc } \\
\text { analysis of } \\
\text { studies 1a-1c }\end{array}$ & nothing & dependent & $\begin{array}{l}\text { dependent constructive } \\
\text { reproducibility }\end{array}$ \\
\hline 250 & JAP & full text & $\begin{array}{l}\text { Johnson, Ilies, \& } \\
\text { Boles (2012) }\end{array}$ & $\begin{array}{l}\text { study la of } \\
\text { previous resarch }\end{array}$ & $\begin{array}{l}\text { replication or } \\
\text { replicate }\end{array}$ & independent & $\begin{array}{l}\text { independent quasi-random } \\
\text { replication }\end{array}$ \\
\hline
\end{tabular}




\begin{tabular}{|c|c|c|c|c|c|c|c|}
\hline \# & Journal & Search & Study & $\begin{array}{l}\text { Which } \\
\text { repetition }\end{array}$ & Terminology & Independence & $\begin{array}{l}\text { Type of replication or } \\
\text { reproducibility study }\end{array}$ \\
\hline 251 & JAP & full text & $\begin{array}{l}\text { Joseph, Jin, } \\
\text { Newman, \& } \\
\text { O'Boyle (2015) }\end{array}$ & & $\begin{array}{l}\text { replication or } \\
\text { replicate }\end{array}$ & $\begin{array}{l}\text { semi- } \\
\text { independent }\end{array}$ & $\begin{array}{l}\text { semi-independent } \\
\text { incremental constructive } \\
\text { reproducibility }\end{array}$ \\
\hline 252 & JAP & full text & $\begin{array}{l}\text { Judge, Rodell, } \\
\text { Klinger, Simon, \& } \\
\text { Crawford (2013) }\end{array}$ & & $\begin{array}{l}\text { replication or } \\
\text { replicate }\end{array}$ & independent & $\begin{array}{l}\text { independent quasi-random } \\
\text { replication }\end{array}$ \\
\hline 253 & JAP & full text & $\begin{array}{l}\text { Kark, Katz-Navon, } \\
\text { \& Delegach (2015) }\end{array}$ & $\begin{array}{l}\text { study } 2 \text { of study } \\
1\end{array}$ & $\begin{array}{l}\text { replication or } \\
\text { replicate }\end{array}$ & dependent & $\begin{array}{l}\text { dependent incremental } \\
\text { constructive replication }\end{array}$ \\
\hline 254 & JAP & full text & $\begin{array}{l}\text { Kark, Katz-Navon, } \\
\text { \& Delegach (2015) }\end{array}$ & $\begin{array}{l}\text { study } 3 \\
\text { supplementary } \\
\text { analyses }\end{array}$ & $\begin{array}{l}\text { replication or } \\
\text { replicate }\end{array}$ & dependent & $\begin{array}{l}\text { dependent quasi-random } \\
\text { reproducibility }\end{array}$ \\
\hline 255 & JAP & full text & $\begin{array}{l}\text { Kark, Katz-Navon, } \\
\text { \& Delegach (2015) }\end{array}$ & $\begin{array}{l}\text { study } 3 \text { of study } \\
2\end{array}$ & $\begin{array}{l}\text { replication or } \\
\text { replicate }\end{array}$ & dependent & $\begin{array}{l}\text { dependent incremental } \\
\text { constructive replication }\end{array}$ \\
\hline 256 & JAP & full text & $\begin{array}{l}\text { Keiser, Sackett, } \\
\text { Kuncel, \& Brothen } \\
(2016)\end{array}$ & $\begin{array}{l}\text { study } 2 \text { sample } \\
2 \text { of sample } 1 \\
\text { and study } 1\end{array}$ & $\begin{array}{l}\text { replication or } \\
\text { replicate }\end{array}$ & dependent & dependent literal replication \\
\hline 257 & JAP & full text & $\begin{array}{l}\text { Keiser, Sackett, } \\
\text { Kuncel, \& Brothen } \\
(2016)\end{array}$ & $\begin{array}{l}\text { study } 2 \text { sample } \\
1 \text { of study } 1\end{array}$ & $\begin{array}{l}\text { replication or } \\
\text { replicate }\end{array}$ & dependent & $\begin{array}{l}\text { dependent confounded } \\
\text { replication }\end{array}$ \\
\hline
\end{tabular}




\begin{tabular}{|c|c|c|c|c|c|c|c|}
\hline \# & Journal & Search & Study & $\begin{array}{l}\text { Which } \\
\text { repetition }\end{array}$ & Terminology & Independence & $\begin{array}{l}\text { Type of replication or } \\
\text { reproducibility study }\end{array}$ \\
\hline 258 & JAP & full text & $\begin{array}{l}\text { Keiser, Sackett, } \\
\text { Kuncel, \& Brothen } \\
\text { (2016) }\end{array}$ & $\begin{array}{l}\text { previous } \\
\text { research }\end{array}$ & $\begin{array}{l}\text { replication or } \\
\text { replicate }\end{array}$ & independent & $\begin{array}{l}\text { independent incremental } \\
\text { constructive replication }\end{array}$ \\
\hline 259 & JAP & full text & $\begin{array}{l}\text { Kiewitz, Restubog, } \\
\text { Shoss, Garcia, \& } \\
\text { Tang (2016) }\end{array}$ & $\begin{array}{l}\text { study } 3 \text { of study } \\
2\end{array}$ & $\begin{array}{l}\text { replication or } \\
\text { replicate }\end{array}$ & dependent & $\begin{array}{l}\text { dependent quasi-random } \\
\text { replication }\end{array}$ \\
\hline 260 & JAP & $\begin{array}{l}\text { abstract } \\
\& \text { title }\end{array}$ & $\begin{array}{l}\text { Kinias \& Sim } \\
(2016)\end{array}$ & $\begin{array}{l}\text { Study } 2 \text { of } \\
\text { Study } 1\end{array}$ & $\begin{array}{l}\text { replication or } \\
\text { replicate }\end{array}$ & dependent & $\begin{array}{l}\text { dependent substantial } \\
\text { constructive replication }\end{array}$ \\
\hline 261 & JAP & full text & $\begin{array}{l}\text { Klein, Dilchert, } \\
\text { Ones, \& Dages } \\
(2015)\end{array}$ & sample 4 & $\begin{array}{l}\text { replication or } \\
\text { replicate }\end{array}$ & dependent & $\begin{array}{l}\text { dependent quasi-random } \\
\text { replication }\end{array}$ \\
\hline 262 & JAP & full text & $\begin{array}{l}\text { Klein, Dilchert, } \\
\text { Ones, \& Dages } \\
(2015)\end{array}$ & sample 3 & nothing & dependent & $\begin{array}{l}\text { dependent quasi-random } \\
\text { replication }\end{array}$ \\
\hline 263 & JAP & full text & $\begin{array}{l}\text { Klein, Dilchert, } \\
\text { Ones, \& Dages } \\
(2015)\end{array}$ & sample 2 & nothing & dependent & $\begin{array}{l}\text { dependent quasi-random } \\
\text { replication }\end{array}$ \\
\hline 264 & JAP & full text & $\begin{array}{l}\text { Kluemper, } \\
\text { McLarty, \& Bing } \\
(2015)\end{array}$ & study 2 of 1 & $\begin{array}{l}\text { replication or } \\
\text { replicate }\end{array}$ & dependent & $\begin{array}{l}\text { dependent quasi-random } \\
\text { replication }\end{array}$ \\
\hline
\end{tabular}




\begin{tabular}{|c|c|c|c|c|c|c|c|}
\hline \# & Journal & Search & Study & $\begin{array}{l}\text { Which } \\
\text { repetition }\end{array}$ & Terminology & Independence & $\begin{array}{l}\text { Type of replication or } \\
\text { reproducibility study }\end{array}$ \\
\hline 265 & JAP & full text & $\begin{array}{l}\text { Kouchaki \& Desai } \\
\text { (2015) }\end{array}$ & $\begin{array}{l}\text { study } 2 \text { of study } \\
1\end{array}$ & $\begin{array}{l}\text { replication or } \\
\text { replicate }\end{array}$ & dependent & $\begin{array}{l}\text { dependent incremental } \\
\text { constructive replication }\end{array}$ \\
\hline 266 & JAP & full text & $\begin{array}{l}\text { Kouchaki \& Desai } \\
(2015)\end{array}$ & $\begin{array}{l}\text { study } 3 \text { of study } \\
1 \text { and } 2\end{array}$ & $\begin{array}{l}\text { replication or } \\
\text { replicate }\end{array}$ & dependent & $\begin{array}{l}\text { dependent incremental } \\
\text { constructive replication }\end{array}$ \\
\hline 267 & JAP & full text & $\begin{array}{l}\text { Kouchaki \& Desai } \\
\text { (2015) }\end{array}$ & $\begin{array}{l}\text { study } 4 \text { of } \\
\text { previous studies }\end{array}$ & $\begin{array}{l}\text { replication or } \\
\text { replicate }\end{array}$ & dependent & $\begin{array}{l}\text { dependent confounded } \\
\text { replication }\end{array}$ \\
\hline 268 & JAP & full text & $\begin{array}{l}\text { Kouchaki \& Desai } \\
(2015)\end{array}$ & $\begin{array}{l}\text { study } 5 \text { of study } \\
3\end{array}$ & $\begin{array}{l}\text { replication or } \\
\text { replicate }\end{array}$ & dependent & $\begin{array}{l}\text { dependent confounded } \\
\text { replication }\end{array}$ \\
\hline 269 & JAP & full text & $\begin{array}{l}\text { Kouchaki \& Desai } \\
(2015)\end{array}$ & study 6 & $\begin{array}{l}\text { replication or } \\
\text { replicate }\end{array}$ & dependent & $\begin{array}{l}\text { dependent confounded } \\
\text { replication }\end{array}$ \\
\hline 270 & JAP & $\begin{array}{l}\text { abstract } \\
\& \text { title }\end{array}$ & $\begin{array}{l}\text { Krumm, Lievens, } \\
\text { Huffmeier, } \\
\text { Lipnevich, Bendels, } \\
\text { \& Hertel (2015) }\end{array}$ & $\begin{array}{l}\text { In study } 1 \text {, } \\
\text { student vs. } \\
\text { working } \\
\text { samples }\end{array}$ & $\begin{array}{l}\text { replication or } \\
\text { replicate }\end{array}$ & dependent & $\begin{array}{l}\text { dependent substantial } \\
\text { constructive replication }\end{array}$ \\
\hline 271 & JAP & $\begin{array}{l}\text { abstract } \\
\& \text { title }\end{array}$ & $\begin{array}{l}\text { Krumm, Lievens, } \\
\text { Huffmeier, } \\
\text { Lipnevich, Bendels, } \\
\text { \& Hertel (2015) }\end{array}$ & study 2 of 1 & $\begin{array}{l}\text { replication or } \\
\text { replicate }\end{array}$ & dependent & $\begin{array}{l}\text { dependent incremental } \\
\text { constructive replication }\end{array}$ \\
\hline 272 & JAP & full text & $\begin{array}{l}\text { Kwong \& Fai } \\
\text { Ellick Wong (2014) }\end{array}$ & $\begin{array}{l}\text { study } 4 \text { of } \\
\text { studies } 1-3\end{array}$ & $\begin{array}{l}\text { replication or } \\
\text { replicate }\end{array}$ & dependent & $\begin{array}{l}\text { dependent quasi-random } \\
\text { replication }\end{array}$ \\
\hline
\end{tabular}




\begin{tabular}{|c|c|c|c|c|c|c|c|}
\hline \# & Journal & Search & Study & $\begin{array}{l}\text { Which } \\
\text { repetition }\end{array}$ & Terminology & Independence & $\begin{array}{l}\text { Type of replication or } \\
\text { reproducibility study }\end{array}$ \\
\hline 273 & JAP & full text & $\begin{array}{l}\text { Kwong \& Fai } \\
\text { Ellick Wong (2014) }\end{array}$ & $\begin{array}{l}\text { study } 3 \text { of study } \\
1 \& 2\end{array}$ & $\begin{array}{l}\text { replication or } \\
\text { replicate }\end{array}$ & dependent & $\begin{array}{l}\text { dependent incremental } \\
\text { constructive replication }\end{array}$ \\
\hline 274 & JAP & full text & $\begin{array}{l}\text { Kwong \& Fai } \\
\text { Ellick Wong (2014) }\end{array}$ & $\begin{array}{l}\text { study } 2 \text { of study } \\
1\end{array}$ & $\begin{array}{l}\text { conceptual } \\
\text { replication }\end{array}$ & dependent & $\begin{array}{l}\text { dependent quasi-random } \\
\text { replication }\end{array}$ \\
\hline 275 & JAP & full text & $\begin{array}{l}\text { Kwong \& Fai } \\
\text { Ellick Wong (2014) }\end{array}$ & $\begin{array}{l}\text { previous } \\
\text { research }\end{array}$ & $\begin{array}{l}\text { replication or } \\
\text { replicate }\end{array}$ & independent & $\begin{array}{l}\text { independent quasi-random } \\
\text { replication }\end{array}$ \\
\hline 276 & JAP & full text & $\begin{array}{l}\text { Lacerenza, Reyes, } \\
\text { Marlow, Joseph, \& } \\
\text { Salas (2017) }\end{array}$ & $\begin{array}{l}\text { previous } \\
\text { research }\end{array}$ & $\begin{array}{l}\text { replication or } \\
\text { replicate }\end{array}$ & independent & $\begin{array}{l}\text { independent quasi-random } \\
\text { replication }\end{array}$ \\
\hline 277 & JAP & full text & $\begin{array}{l}\text { Lambert, Tepper, } \\
\text { Carr, Holt, \& } \\
\text { Barelka (2012) }\end{array}$ & study 2 of study & $\begin{array}{l}\text { replication or } \\
\text { replicate }\end{array}$ & dependent & $\begin{array}{l}\text { dependent incremental } \\
\text { constructive replication }\end{array}$ \\
\hline 278 & JAP & full text & $\begin{array}{l}\text { Lanaj, Johnson, \& } \\
\text { Lee (2016) }\end{array}$ & $\begin{array}{l}\text { study } 2 \text { of study } \\
1\end{array}$ & $\begin{array}{l}\text { replication or } \\
\text { replicate }\end{array}$ & dependent & $\begin{array}{l}\text { dependent quasi-random } \\
\text { replication }\end{array}$ \\
\hline 279 & JAP & full text & $\begin{array}{l}\text { Le, Robbins, \& } \\
\text { Westrick (2014) }\end{array}$ & $\begin{array}{l}\text { study } 2 \text { of study } \\
1\end{array}$ & $\begin{array}{l}\text { replication or } \\
\text { replicate }\end{array}$ & dependent & generalizability \\
\hline 280 & JAP & full text & $\begin{array}{l}\text { Lee, Gino, \& Staats } \\
(2014)\end{array}$ & $\begin{array}{l}\text { study } 2 \text { of study } \\
1\end{array}$ & $\begin{array}{l}\text { constructive } \\
\text { replication }\end{array}$ & dependent & $\begin{array}{l}\text { dependent quasi-random } \\
\text { replication }\end{array}$ \\
\hline 281 & JAP & full text & Leroy et al. (2012) & & $\begin{array}{l}\text { replication or } \\
\text { replicate }\end{array}$ & dependent & $\begin{array}{l}\text { dependent quasi-random } \\
\text { reproducibility }\end{array}$ \\
\hline
\end{tabular}




\begin{tabular}{|c|c|c|c|c|c|c|c|}
\hline \# & Journal & Search & Study & $\begin{array}{l}\text { Which } \\
\text { repetition }\end{array}$ & Terminology & Independence & $\begin{array}{l}\text { Type of replication or } \\
\text { reproducibility study }\end{array}$ \\
\hline 282 & JAP & $\begin{array}{l}\text { abstract } \\
\& \text { title }\end{array}$ & $\begin{array}{l}\text { Levi \& Fried } \\
(2008)\end{array}$ & $\begin{array}{l}\text { Study } 1 \text { of } \\
\text { previous }\end{array}$ & $\begin{array}{l}\text { replication or } \\
\text { replicate }\end{array}$ & independent & $\begin{array}{l}\text { independent quasi-random } \\
\text { replication }\end{array}$ \\
\hline 283 & JAP & full text & $\begin{array}{l}\text { Li, Lee, Mitchell, } \\
\text { Hom, \& Griffeth } \\
\text { (2016) }\end{array}$ & $\begin{array}{l}\text { study } 2 \text { of study } \\
1\end{array}$ & $\begin{array}{l}\text { replication or } \\
\text { replicate }\end{array}$ & dependent & $\begin{array}{l}\text { dependent quasi-random } \\
\text { replication }\end{array}$ \\
\hline 284 & JAP & full text & $\begin{array}{l}\text { Lian, Ferris, \& } \\
\text { Brown (2012) }\end{array}$ & study 3 & nothing & dependent & $\begin{array}{l}\text { dependent incremental } \\
\text { constructive replication }\end{array}$ \\
\hline 285 & JAP & full text & $\begin{array}{l}\text { Lian, Ferris, \& } \\
\text { Brown (2012) }\end{array}$ & $\begin{array}{l}\text { study } 2 \text { of study } \\
1\end{array}$ & $\begin{array}{l}\text { replication or } \\
\text { replicate }\end{array}$ & dependent & $\begin{array}{l}\text { dependent incremental } \\
\text { constructive replication }\end{array}$ \\
\hline 286 & JAP & full text & $\begin{array}{l}\text { Lievens, Schollaert, } \\
\text { \& Keen (2015) }\end{array}$ & study 4 & nothing & dependent & $\begin{array}{l}\text { dependent quasi-random } \\
\text { replication }\end{array}$ \\
\hline 287 & JAP & full text & $\begin{array}{l}\text { Lievens, Schollaert, } \\
\text { \& Keen (2015) }\end{array}$ & $\begin{array}{l}\text { study } 2 \text { of study } \\
1\end{array}$ & $\begin{array}{l}\text { replication or } \\
\text { replicate }\end{array}$ & dependent & $\begin{array}{l}\text { dependent incremental } \\
\text { constructive replication }\end{array}$ \\
\hline 288 & JAP & full text & $\begin{array}{l}\text { Lievens, Schollaert, } \\
\text { \& Keen (2015) }\end{array}$ & $\begin{array}{l}\text { study } 3 \text { of study } \\
1 \text { and } 2\end{array}$ & $\begin{array}{l}\text { replication or } \\
\text { replicate }\end{array}$ & dependent & $\begin{array}{l}\text { dependent confounded } \\
\text { replication }\end{array}$ \\
\hline 289 & JAP & full text & $\begin{array}{l}\text { Lin \& Johnson } \\
(2015)\end{array}$ & $\begin{array}{l}\text { study } 2 \text { of study } \\
1\end{array}$ & $\begin{array}{l}\text { replication or } \\
\text { replicate }\end{array}$ & dependent & $\begin{array}{l}\text { dependent quasi-random } \\
\text { replication }\end{array}$ \\
\hline 290 & JAP & full text & $\begin{array}{l}\text { Lin \& Johnson } \\
(2015)\end{array}$ & $\begin{array}{l}\text { study } 2 \text { of study } \\
1\end{array}$ & $\begin{array}{l}\text { replication or } \\
\text { replicate }\end{array}$ & dependent & $\begin{array}{l}\text { dependent incremental } \\
\text { constructive replication }\end{array}$ \\
\hline
\end{tabular}




\begin{tabular}{|c|c|c|c|c|c|c|c|}
\hline \# & Journal & Search & Study & $\begin{array}{l}\text { Which } \\
\text { repetition }\end{array}$ & Terminology & Independence & $\begin{array}{l}\text { Type of replication or } \\
\text { reproducibility study }\end{array}$ \\
\hline 291 & JAP & $\begin{array}{l}\text { abstract } \\
\& \text { title }\end{array}$ & $\begin{array}{l}\text { Lindsey, Avery, } \\
\text { Dawson, \& King } \\
(2017)\end{array}$ & $\begin{array}{l}2008-2009 \text { data } \\
\text { as replication of } \\
\text { the } 2009-2010 \\
\text { data }\end{array}$ & $\begin{array}{l}\text { replication or } \\
\text { replicate }\end{array}$ & dependent & dependent literal replication \\
\hline 292 & JAP & $\begin{array}{l}\text { abstract } \\
\& \text { title }\end{array}$ & $\begin{array}{l}\text { Lindsey, Avery, } \\
\text { Dawson, \& King } \\
\text { (2017) }\end{array}$ & $\begin{array}{l}2007-2008 \text { data } \\
\text { as replication of } \\
\text { the } 2009-2010 \\
\text { data }\end{array}$ & $\begin{array}{l}\text { replication or } \\
\text { replicate }\end{array}$ & dependent & dependent literal replication \\
\hline 293 & JAP & $\begin{array}{l}\text { abstract } \\
\& \text { title }\end{array}$ & $\begin{array}{l}\text { Lindsey, Avery, } \\
\text { Dawson, \& King } \\
(2017)\end{array}$ & $\begin{array}{l}\text { study } 2 \text { of study } \\
1\end{array}$ & $\begin{array}{l}\text { constructive } \\
\text { replication }\end{array}$ & dependent & $\begin{array}{l}\text { Dependent regressive } \\
\text { replication }\end{array}$ \\
\hline 294 & JAP & $\begin{array}{l}\text { abstract } \\
\& \text { title }\end{array}$ & $\begin{array}{l}\text { Lindsey, Avery, } \\
\text { Dawson, \& King } \\
(2017)\end{array}$ & $\begin{array}{l}\text { study } 3 \text { of } \\
\text { studies } 1 \text { and } 2\end{array}$ & $\begin{array}{l}\text { replication or } \\
\text { replicate }\end{array}$ & dependent & $\begin{array}{l}\text { dependent confounded } \\
\text { replication }\end{array}$ \\
\hline 295 & JAP & $\begin{array}{l}\text { abstract } \\
\& \text { title }\end{array}$ & $\begin{array}{l}\text { Liu, Tangirala, } \\
\text { Lam, Chen, Jia, \& } \\
\text { Huang (2015) }\end{array}$ & Study 2 of 1 & $\begin{array}{l}\text { replication or } \\
\text { replicate }\end{array}$ & dependent & $\begin{array}{l}\text { dependent confounded } \\
\text { replication }\end{array}$ \\
\hline 296 & JAP & full text & $\begin{array}{l}\text { Liu, Gong, \& Liu } \\
\text { (2014) }\end{array}$ & $\begin{array}{l}\text { previous } \\
\text { research }\end{array}$ & $\begin{array}{l}\text { constructive } \\
\text { replication }\end{array}$ & independent & $\begin{array}{l}\text { independent quasi-random } \\
\text { replication }\end{array}$ \\
\hline 297 & JAP & full text & $\begin{array}{l}\text { Long \& Christian } \\
\text { (2015) }\end{array}$ & $\begin{array}{l}\text { study } 2 \text { of study } \\
1\end{array}$ & $\begin{array}{l}\text { constructive } \\
\text { replication }\end{array}$ & dependent & $\begin{array}{l}\text { dependent confounded } \\
\text { replication }\end{array}$ \\
\hline
\end{tabular}




\begin{tabular}{|c|c|c|c|c|c|c|c|}
\hline$\#$ & Journal & Search & Study & $\begin{array}{l}\text { Which } \\
\text { repetition }\end{array}$ & Terminology & Independence & $\begin{array}{l}\text { Type of replication or } \\
\text { reproducibility study }\end{array}$ \\
\hline 298 & JAP & $\begin{array}{l}\text { abstract } \\
\& \text { title }\end{array}$ & $\begin{array}{l}\text { Loschelder, } \\
\text { Trotschel, Swaab, } \\
\text { Friese, \& Galinsky } \\
2016\end{array}$ & $\begin{array}{l}\text { Study } 1 \text { of } \\
\text { previous } \\
\text { research }\end{array}$ & $\begin{array}{l}\text { replication or } \\
\text { replicate }\end{array}$ & independent & generalizability \\
\hline 299 & JAP & $\begin{array}{l}\text { abstract } \\
\& \text { title }\end{array}$ & $\begin{array}{l}\text { Loschelder, } \\
\text { Trotschel, Swaab, } \\
\text { Friese, \& Galinsky } \\
2016\end{array}$ & $\begin{array}{l}\text { Study } 3 \text { of } \\
\text { Studies } 1 \text { and } 2 \\
\text { and previous } \\
\text { research }\end{array}$ & $\begin{array}{l}\text { replication or } \\
\text { replicate }\end{array}$ & dependent & $\begin{array}{l}\text { dependent incremental } \\
\text { constructive replication }\end{array}$ \\
\hline 300 & JAP & $\begin{array}{l}\text { abstract } \\
\& \text { title }\end{array}$ & $\begin{array}{l}\text { Loschelder, } \\
\text { Trotschel, Swaab, } \\
\text { Friese, \& Galinsky } \\
2016\end{array}$ & $\begin{array}{l}\text { Study } 2 \text { of } \\
\text { Study } 1 \text { and } \\
\text { previous } \\
\text { research }\end{array}$ & $\begin{array}{l}\text { replication or } \\
\text { replicate }\end{array}$ & dependent & $\begin{array}{l}\text { dependent incremental } \\
\text { constructive replication }\end{array}$ \\
\hline 301 & JAP & $\begin{array}{l}\text { abstract } \\
\& \text { title }\end{array}$ & $\begin{array}{l}\text { Luchak \& Gellatly } \\
\text { (2007) }\end{array}$ & $\begin{array}{l}\text { Study } 1 \text { of } \\
\text { previous }\end{array}$ & $\begin{array}{l}\text { replication or } \\
\text { replicate }\end{array}$ & independent & $\begin{array}{l}\text { independent incremental } \\
\text { constructive replication }\end{array}$ \\
\hline 302 & JAP & $\begin{array}{l}\text { abstract } \\
\& \text { title }\end{array}$ & $\begin{array}{l}\text { Luchak \& Gellatly } \\
\text { (2007) }\end{array}$ & $\begin{array}{l}\text { Study } 2 \text { of } \\
\text { previous }\end{array}$ & $\begin{array}{l}\text { replication or } \\
\text { replicate }\end{array}$ & independent & $\begin{array}{l}\text { independent incremental } \\
\text { constructive replication }\end{array}$ \\
\hline 303 & JAP & $\begin{array}{l}\text { abstract } \\
\& \text { title }\end{array}$ & $\begin{array}{l}\text { Luchak \& Gellatly } \\
\text { (2007) }\end{array}$ & $\begin{array}{l}\text { Study } 3 \text { of } \\
\text { previous }\end{array}$ & $\begin{array}{l}\text { replication or } \\
\text { replicate }\end{array}$ & independent & $\begin{array}{l}\text { independent incremental } \\
\text { constructive replication }\end{array}$ \\
\hline 304 & JAP & full text & $\begin{array}{l}\text { Luksyte, Avery, \& } \\
\text { Yeo (2015) }\end{array}$ & $\begin{array}{l}\text { study } 2 \text { of study } \\
1\end{array}$ & $\begin{array}{l}\text { constructive } \\
\text { replication }\end{array}$ & dependent & $\begin{array}{l}\text { dependent confounded } \\
\text { replication }\end{array}$ \\
\hline
\end{tabular}




\begin{tabular}{|c|c|c|c|c|c|c|c|}
\hline \# & Journal & Search & Study & $\begin{array}{l}\text { Which } \\
\text { repetition }\end{array}$ & Terminology & Independence & $\begin{array}{l}\text { Type of replication or } \\
\text { reproducibility study }\end{array}$ \\
\hline 305 & JAP & full text & $\begin{array}{l}\text { Lyons, Volpone, } \\
\text { Wessel, \& Alonso } \\
\text { (2017) }\end{array}$ & $\begin{array}{l}\text { study } 2 \text { of study } \\
1\end{array}$ & $\begin{array}{l}\text { replication or } \\
\text { replicate }\end{array}$ & dependent & $\begin{array}{l}\text { dependent incremental } \\
\text { constructive replication }\end{array}$ \\
\hline 306 & JAP & full text & $\begin{array}{l}\text { Madera \& Hebl } \\
(2012)\end{array}$ & $\begin{array}{l}\text { study } 2 \text { of study } \\
1\end{array}$ & $\begin{array}{l}\text { replication or } \\
\text { replicate }\end{array}$ & dependent & $\begin{array}{l}\text { dependent quasi-random } \\
\text { replication }\end{array}$ \\
\hline 307 & JAP & full text & $\begin{array}{l}\text { Madrid, Totterdell, } \\
\text { Niven, \& Barros } \\
(2016)\end{array}$ & $\begin{array}{l}\text { study } 2 \text { of study } \\
1\end{array}$ & $\begin{array}{l}\text { replication or } \\
\text { replicate }\end{array}$ & dependent & $\begin{array}{l}\text { dependent quasi-random } \\
\text { replication }\end{array}$ \\
\hline 308 & JAP & full text & $\begin{array}{l}\text { Madrid, Totterdell, } \\
\text { Niven, \& Barros } \\
\text { (2016) }\end{array}$ & $\begin{array}{l}\text { previous } \\
\text { research }\end{array}$ & $\begin{array}{l}\text { replication or } \\
\text { replicate }\end{array}$ & independent & $\begin{array}{l}\text { independent confounded } \\
\text { replication }\end{array}$ \\
\hline 309 & JAP & full text & $\begin{array}{l}\text { Mai, Ellis, } \\
\text { Christian, \& Porter } \\
\text { (2016) }\end{array}$ & $\begin{array}{l}\text { study } 2 \text { of study } \\
1\end{array}$ & $\begin{array}{l}\text { replication or } \\
\text { replicate }\end{array}$ & dependent & $\begin{array}{l}\text { dependent incremental } \\
\text { constructive replication }\end{array}$ \\
\hline 310 & JAP & $\begin{array}{l}\text { abstract } \\
\& \text { title }\end{array}$ & $\begin{array}{l}\text { Martinez, Sawyer, } \\
\text { Thoroughgood, } \\
\text { Ruggs, \& Smith } \\
2017\end{array}$ & Appendix A & $\begin{array}{l}\text { replication or } \\
\text { replicate }\end{array}$ & dependent & $\begin{array}{l}\text { dependent constructive } \\
\text { reproducibility }\end{array}$ \\
\hline 311 & JAP & $\begin{array}{l}\text { abstract } \\
\& \text { title }\end{array}$ & $\begin{array}{l}\text { Martinez, Sawyer, } \\
\text { Thoroughgood, } \\
\text { Ruggs, \& Smith } \\
2017\end{array}$ & $\begin{array}{l}\text { Study } 2 \text { of stude } \\
1\end{array}$ & $\begin{array}{l}\text { replicate and } \\
\text { extend }\end{array}$ & dependent & dependent literal replication \\
\hline
\end{tabular}




\begin{tabular}{|c|c|c|c|c|c|c|c|}
\hline \# & Journal & Search & Study & $\begin{array}{l}\text { Which } \\
\text { repetition }\end{array}$ & Terminology & Independence & $\begin{array}{l}\text { Type of replication or } \\
\text { reproducibility study }\end{array}$ \\
\hline 312 & JAP & $\begin{array}{l}\text { abstract } \\
\& \text { title }\end{array}$ & $\begin{array}{l}\text { Martinez, Sawyer, } \\
\text { Thoroughgood, } \\
\text { Ruggs, \& Smith } \\
2017\end{array}$ & footnote 3 & $\begin{array}{l}\text { replication or } \\
\text { replicate }\end{array}$ & dependent & $\begin{array}{l}\text { dependent confounded } \\
\text { replication }\end{array}$ \\
\hline 313 & JAP & full text & $\begin{array}{l}\text { Martinez, White, } \\
\text { Shapiro, \& Hebl } \\
\text { (2016) }\end{array}$ & $\begin{array}{l}\text { previous } \\
\text { research }\end{array}$ & $\begin{array}{l}\text { replication or } \\
\text { replicate }\end{array}$ & independent & $\begin{array}{l}\text { independent quasi-random } \\
\text { replication }\end{array}$ \\
\hline 314 & JAP & $\begin{array}{l}\text { abstract } \\
\& \text { title }\end{array}$ & $\begin{array}{l}\text { Mathieu, } \\
\text { Kukenberger, } \\
\text { D'Innocenzo, \& } \\
\text { Reilly (2015) }\end{array}$ & study 2 of 1 & $\begin{array}{l}\text { replication or } \\
\text { replicate }\end{array}$ & dependent & $\begin{array}{l}\text { dependent substantial } \\
\text { constructive replication }\end{array}$ \\
\hline 315 & JAP & full text & $\begin{array}{l}\text { Maynard, Luciano, } \\
\text { D'Innocenzo, } \\
\text { Mathieu, \& Dean } \\
\text { (2014) }\end{array}$ & $\begin{array}{l}\text { previous } \\
\text { research }\end{array}$ & $\begin{array}{l}\text { replication or } \\
\text { replicate }\end{array}$ & independent & $\begin{array}{l}\text { independent quasi-random } \\
\text { replication }\end{array}$ \\
\hline 316 & JAP & full text & $\begin{array}{l}\text { McCarthy, } \\
\text { Trougakos, \& } \\
\text { Cheng (2016) }\end{array}$ & & $\begin{array}{l}\text { replication or } \\
\text { replicate }\end{array}$ & dependent & $\begin{array}{l}\text { dependent quasi-random } \\
\text { reproducibility }\end{array}$ \\
\hline 317 & JAP & full text & $\begin{array}{l}\text { McGonagle, Fisher, } \\
\text { Barnes-Farrell, \& } \\
\text { Grosch }(2015)\end{array}$ & $\begin{array}{l}\text { sample } 2 \text { and } 3 \\
\text { of sample } 1\end{array}$ & $\begin{array}{l}\text { replication or } \\
\text { replicate }\end{array}$ & dependent & $\begin{array}{l}\text { dependent quasi-random } \\
\text { replication }\end{array}$ \\
\hline
\end{tabular}




\begin{tabular}{|c|c|c|c|c|c|c|c|}
\hline \# & Journal & Search & Study & $\begin{array}{l}\text { Which } \\
\text { repetition }\end{array}$ & Terminology & Independence & $\begin{array}{l}\text { Type of replication or } \\
\text { reproducibility study }\end{array}$ \\
\hline 318 & JAP & full text & $\begin{array}{l}\text { McGonagle, Fisher, } \\
\text { Barnes-Farrell, \& } \\
\text { Grosch }(2015)\end{array}$ & $\begin{array}{l}\text { sample } 2 \text { of } \\
\text { sample } 1\end{array}$ & $\begin{array}{l}\text { replication or } \\
\text { replicate }\end{array}$ & dependent & $\begin{array}{l}\text { dependent quasi-random } \\
\text { replication }\end{array}$ \\
\hline 319 & JAP & full text & $\begin{array}{l}\text { McGonagle, Fisher, } \\
\text { Barnes-Farrell, \& } \\
\text { Grosch }(2015)\end{array}$ & $\begin{array}{l}\text { previous } \\
\text { research }\end{array}$ & $\begin{array}{l}\text { replication or } \\
\text { replicate }\end{array}$ & independent & $\begin{array}{l}\text { independent quasi-random } \\
\text { replication }\end{array}$ \\
\hline 320 & JAP & full text & $\begin{array}{l}\text { Melwani, Mueller, } \\
\text { \& Overbeck (2012) }\end{array}$ & $\begin{array}{l}\text { study } 1 \mathrm{~b} \text { of } \\
\text { study } 1 \mathrm{a}\end{array}$ & $\begin{array}{l}\text { constructive } \\
\text { replication }\end{array}$ & dependent & $\begin{array}{l}\text { dependent quasi-random } \\
\text { replication }\end{array}$ \\
\hline 321 & JAP & full text & $\begin{array}{l}\text { Melwani, Mueller, } \\
\text { \& Overbeck (2012) }\end{array}$ & $\begin{array}{l}\text { study } 2 \text { of study } \\
1 \text { a and } 1 b\end{array}$ & $\begin{array}{l}\text { constructive } \\
\text { replication }\end{array}$ & dependent & $\begin{array}{l}\text { dependent quasi-random } \\
\text { replication }\end{array}$ \\
\hline 322 & JAP & full text & $\begin{array}{l}\text { Mitchell \& } \\
\text { Ambrose (2012) }\end{array}$ & $\begin{array}{l}\text { study } 2 \text { of study } \\
1\end{array}$ & $\begin{array}{l}\text { replication or } \\
\text { replicate }\end{array}$ & dependent & $\begin{array}{l}\text { dependent quasi-random } \\
\text { replication }\end{array}$ \\
\hline 323 & JAP & full text & $\begin{array}{l}\text { Mitchell \& } \\
\text { Ambrose (2012) }\end{array}$ & study 3 & $\begin{array}{l}\text { generalizabilit } \\
\mathrm{y}\end{array}$ & dependent & $\begin{array}{l}\text { dependent quasi-random } \\
\text { replication }\end{array}$ \\
\hline 324 & JAP & $\begin{array}{l}\text { abstract } \\
\& \text { title }\end{array}$ & $\begin{array}{l}\text { Nahum-Shani, } \\
\text { Henderson, Lim, \& } \\
\text { Vinokur (2014) }\end{array}$ & Study 2 of 1 & $\begin{array}{l}\text { replication or } \\
\text { replicate }\end{array}$ & dependent & $\begin{array}{l}\text { dependent quasi-random } \\
\text { replication }\end{array}$ \\
\hline 325 & JAP & $\begin{array}{l}\text { abstract } \\
\& \text { title }\end{array}$ & $\begin{array}{l}\text { Nahum-Shani, } \\
\text { Henderson, Lim, \& } \\
\text { Vinokur (2014) }\end{array}$ & $\begin{array}{l}\text { Study } 3 \text { of } 1 \\
\text { and } 2\end{array}$ & $\begin{array}{l}\text { replication or } \\
\text { replicate }\end{array}$ & dependent & $\begin{array}{l}\text { dependent confounded } \\
\text { replication }\end{array}$ \\
\hline
\end{tabular}




\begin{tabular}{|c|c|c|c|c|c|c|c|}
\hline \# & Journal & Search & Study & $\begin{array}{l}\text { Which } \\
\text { repetition }\end{array}$ & Terminology & Independence & $\begin{array}{l}\text { Type of replication or } \\
\text { reproducibility study }\end{array}$ \\
\hline 326 & JAP & $\begin{array}{l}\text { abstract } \\
\& \text { title }\end{array}$ & $\begin{array}{l}\text { Nahum-Shani, } \\
\text { Henderson, Lim, \& } \\
\text { Vinokur (2014) }\end{array}$ & $\begin{array}{l}\text { reanalysis of } \\
\text { study } 1\end{array}$ & $\begin{array}{l}\text { replication or } \\
\text { replicate }\end{array}$ & dependent & $\begin{array}{l}\text { dependent constructive } \\
\text { reproducibility }\end{array}$ \\
\hline 327 & JAP & full text & $\begin{array}{l}\text { Naveh \& Katz- } \\
\text { Navon (2015) }\end{array}$ & & $\begin{array}{l}\text { replication or } \\
\text { replicate }\end{array}$ & dependent & $\begin{array}{l}\text { dependent quasi-random } \\
\text { reproducibility }\end{array}$ \\
\hline 328 & JAP & full text & $\begin{array}{l}\text { Niessen \& } \\
\text { Jimmieson (2016) }\end{array}$ & $\begin{array}{l}\text { previous } \\
\text { research }\end{array}$ & $\begin{array}{l}\text { replication or } \\
\text { replicate }\end{array}$ & independent & $\begin{array}{l}\text { independent quasi-random } \\
\text { replication }\end{array}$ \\
\hline 329 & JAP & full text & $\begin{array}{l}\text { Nye, Brummel, \& } \\
\text { Drasgow (2014) }\end{array}$ & $\begin{array}{l}\text { study } 3 \text { of study } \\
1 \text { and } 2\end{array}$ & $\begin{array}{l}\text { replication or } \\
\text { replicate }\end{array}$ & dependent & $\begin{array}{l}\text { dependent quasi-random } \\
\text { replication }\end{array}$ \\
\hline 330 & JAP & full text & $\begin{array}{l}\text { Nye, Brummel, \& } \\
\text { Drasgow (2014) }\end{array}$ & $\begin{array}{l}\text { previous } \\
\text { research }\end{array}$ & $\begin{array}{l}\text { replication or } \\
\text { replicate }\end{array}$ & independent & $\begin{array}{l}\text { independent quasi-random } \\
\text { replication }\end{array}$ \\
\hline 331 & JAP & full text & $\begin{array}{l}\text { Nye, Brummel, \& } \\
\text { Drasgow (2014) }\end{array}$ & $\begin{array}{l}\text { study } 2 \text { of study } \\
1\end{array}$ & $\begin{array}{l}\text { replication or } \\
\text { replicate }\end{array}$ & dependent & $\begin{array}{l}\text { dependent quasi-random } \\
\text { replication }\end{array}$ \\
\hline 332 & JAP & full text & $\begin{array}{l}\text { Oc, Bashshur, \& } \\
\text { Moore (2015) }\end{array}$ & $\begin{array}{l}\text { study } 2 \text { of study } \\
1\end{array}$ & $\begin{array}{l}\text { replication or } \\
\text { replicate }\end{array}$ & dependent & $\begin{array}{l}\text { dependent quasi-random } \\
\text { replication }\end{array}$ \\
\hline 333 & JAP & full text & $\begin{array}{l}\text { O'Reilly, Aquino, } \\
\text { \& Skarlicki (2016) }\end{array}$ & $\begin{array}{l}\text { study } 1 \mathrm{~b} \text { of } \\
\text { study } 1 \mathrm{a}\end{array}$ & $\begin{array}{l}\text { replication or } \\
\text { replicate }\end{array}$ & dependent & generalizability \\
\hline 334 & JAP & full text & $\begin{array}{l}\text { O'Reilly, Aquino, } \\
\text { \& Skarlicki (2016) }\end{array}$ & $\begin{array}{l}\text { study } 2 \text { of study } \\
1 \mathrm{a} \text { and } 1 \mathrm{~b}\end{array}$ & $\begin{array}{l}\text { replication or } \\
\text { replicate }\end{array}$ & dependent & $\begin{array}{l}\text { dependent quasi-random } \\
\text { replication }\end{array}$ \\
\hline
\end{tabular}




\begin{tabular}{|c|c|c|c|c|c|c|c|}
\hline \# & Journal & Search & Study & $\begin{array}{l}\text { Which } \\
\text { repetition }\end{array}$ & Terminology & Independence & $\begin{array}{l}\text { Type of replication or } \\
\text { reproducibility study }\end{array}$ \\
\hline 335 & JAP & $\begin{array}{l}\text { abstract } \\
\text { \& title }\end{array}$ & $\begin{array}{l}\text { Owens, Baker, } \\
\text { Sumpter, \& } \\
\text { Cameron (2016) }\end{array}$ & Study 4 of 3 & $\begin{array}{l}\text { replication or } \\
\text { replicate }\end{array}$ & dependent & $\begin{array}{l}\text { dependent substantial } \\
\text { constructive replication }\end{array}$ \\
\hline 336 & JAP & full text & $\begin{array}{l}\text { Pearsall \& } \\
\text { Venkataramani } \\
(2015)\end{array}$ & $\begin{array}{l}\text { previous } \\
\text { research }\end{array}$ & $\begin{array}{l}\text { replication or } \\
\text { replicate }\end{array}$ & independent & $\begin{array}{l}\text { independent quasi-random } \\
\text { replication }\end{array}$ \\
\hline 337 & JAP & full text & Perry-Smith (2014) & $\begin{array}{l}\text { study } 2 \text { of study } \\
1\end{array}$ & $\begin{array}{l}\text { replication or } \\
\text { replicate }\end{array}$ & dependent & $\begin{array}{l}\text { dependent incremental } \\
\text { constructive replication }\end{array}$ \\
\hline 338 & JAP & full text & $\begin{array}{l}\text { Pitesa \& Thau } \\
(2013)\end{array}$ & $\begin{array}{l}\text { supplementary } \\
\text { data collection } \\
\text { for study } 1\end{array}$ & $\begin{array}{l}\text { constructive } \\
\text { replication }\end{array}$ & dependent & $\begin{array}{l}\text { dependent quasi-random } \\
\text { replication }\end{array}$ \\
\hline 339 & JAP & full text & $\begin{array}{l}\text { Pitesa \& Thau } \\
(2013)\end{array}$ & $\begin{array}{l}\text { supplementary } \\
\text { data collection } \\
\text { for study } 2\end{array}$ & $\begin{array}{l}\text { constructive } \\
\text { replication }\end{array}$ & dependent & $\begin{array}{l}\text { dependent incremental } \\
\text { constructive replication }\end{array}$ \\
\hline 340 & JAP & full text & $\begin{array}{l}\text { Pitesa \& Thau } \\
(2013)\end{array}$ & study 2 & nothing & dependent & $\begin{array}{l}\text { dependent incremental } \\
\text { constructive replication }\end{array}$ \\
\hline 341 & JAP & full text & $\begin{array}{l}\text { Pitesa \& Thau } \\
(2013)\end{array}$ & $\begin{array}{l}\text { study } 3 \text { of } \\
\text { studies } 1 \text { and } 2\end{array}$ & $\begin{array}{l}\text { constructive } \\
\text { replication }\end{array}$ & dependent & $\begin{array}{l}\text { dependent confounded } \\
\text { replication }\end{array}$ \\
\hline 342 & JAP & full text & $\begin{array}{l}\text { Porath, Gerbasi, \& } \\
\text { Schorch (2015) }\end{array}$ & $\begin{array}{l}\text { study } 2 \text { of study } \\
1\end{array}$ & $\begin{array}{l}\text { replication or } \\
\text { replicate }\end{array}$ & dependent & $\begin{array}{l}\text { dependent confounded } \\
\text { replication }\end{array}$ \\
\hline
\end{tabular}




\begin{tabular}{|c|c|c|c|c|c|c|c|}
\hline \# & Journal & Search & Study & $\begin{array}{l}\text { Which } \\
\text { repetition }\end{array}$ & Terminology & Independence & $\begin{array}{l}\text { Type of replication or } \\
\text { reproducibility study }\end{array}$ \\
\hline 343 & JAP & full text & $\begin{array}{l}\text { Qin, Ren, Zhang, \& } \\
\text { Johnson (2015) }\end{array}$ & & $\begin{array}{l}\text { replication or } \\
\text { replicate }\end{array}$ & dependent & $\begin{array}{l}\text { dependent quasi-random } \\
\text { reproducibility }\end{array}$ \\
\hline 344 & JAP & full text & $\begin{array}{l}\text { Qin, Ren, Zhang, \& } \\
\text { Johnson (2015) }\end{array}$ & $\begin{array}{l}\text { study } 3 \text { of } \\
\text { studies } 1 \text { and } 2\end{array}$ & $\begin{array}{l}\text { replication or } \\
\text { replicate }\end{array}$ & dependent & $\begin{array}{l}\text { dependent incremental } \\
\text { constructive replication }\end{array}$ \\
\hline 345 & JAP & full text & $\begin{array}{l}\text { Rafaeli, Erez, } \\
\text { Ravid, Derfler- } \\
\text { Rozin, Treister, \& } \\
\text { Scheyer (2012) }\end{array}$ & $\begin{array}{l}\text { study } 3 \text { of study } \\
1 \text { and } 2\end{array}$ & $\begin{array}{l}\text { replication or } \\
\text { replicate }\end{array}$ & dependent & $\begin{array}{l}\text { dependent quasi-random } \\
\text { replication }\end{array}$ \\
\hline 346 & JAP & full text & $\begin{array}{l}\text { Rafaeli, Erez, } \\
\text { Ravid, Derfler- } \\
\text { Rozin, Treister, \& } \\
\text { Scheyer (2012) }\end{array}$ & $\begin{array}{l}\text { study } 2 \text { of study } \\
1\end{array}$ & $\begin{array}{l}\text { replication or } \\
\text { replicate }\end{array}$ & dependent & $\begin{array}{l}\text { dependent quasi-random } \\
\text { replication }\end{array}$ \\
\hline 347 & JAP & $\begin{array}{l}\text { abstract } \\
\& \text { title }\end{array}$ & $\begin{array}{l}\text { Reb \& Greguras } \\
(2010)\end{array}$ & $\begin{array}{l}\text { study } 2 \text { of study } \\
1\end{array}$ & $\begin{array}{l}\text { replication or } \\
\text { replicate }\end{array}$ & dependent & $\begin{array}{l}\text { dependent incremental } \\
\text { constructive replication }\end{array}$ \\
\hline 348 & JAP & $\begin{array}{l}\text { abstract } \\
\& \text { title }\end{array}$ & $\begin{array}{l}\text { Reb \& Greguras } \\
(2010)\end{array}$ & $\begin{array}{l}\text { study } 1 \text { of } \\
\text { previous } \\
\text { research }\end{array}$ & $\begin{array}{l}\text { replication or } \\
\text { replicate }\end{array}$ & $\begin{array}{l}\text { Semi- } \\
\text { independent }\end{array}$ & $\begin{array}{l}\text { semi-independent quasi } \\
\text { random replication }\end{array}$ \\
\hline 349 & JAP & full text & $\begin{array}{l}\text { Reich \& } \\
\text { Hershcovis (2015) }\end{array}$ & $\begin{array}{l}\text { study } 2 \text { of study } \\
1\end{array}$ & $\begin{array}{l}\text { replication or } \\
\text { replicate }\end{array}$ & dependent & $\begin{array}{l}\text { dependent incremental } \\
\text { constructive replication }\end{array}$ \\
\hline
\end{tabular}




\begin{tabular}{|c|c|c|c|c|c|c|c|}
\hline \# & Journal & Search & Study & $\begin{array}{l}\text { Which } \\
\text { repetition }\end{array}$ & Terminology & Independence & $\begin{array}{l}\text { Type of replication or } \\
\text { reproducibility study }\end{array}$ \\
\hline 350 & JAP & full text & $\begin{array}{l}\text { Ritter, Matthews, } \\
\text { Ford, \& Henderson } \\
\text { (2016) }\end{array}$ & $\begin{array}{l}\text { previous } \\
\text { research }\end{array}$ & $\begin{array}{l}\text { replication or } \\
\text { replicate }\end{array}$ & independent & $\begin{array}{l}\text { independent quasi-random } \\
\text { replication }\end{array}$ \\
\hline 351 & JAP & full text & $\begin{array}{l}\text { Rockmann \& } \\
\text { Ballinger (2017) }\end{array}$ & $\begin{array}{l}\text { study } 2 \text { of study } \\
1\end{array}$ & $\begin{array}{l}\text { replication or } \\
\text { replicate }\end{array}$ & dependent & $\begin{array}{l}\text { dependent quasi-random } \\
\text { constructive replication }\end{array}$ \\
\hline 352 & JAP & $\begin{array}{l}\text { abstract } \\
\& \text { title }\end{array}$ & $\begin{array}{l}\text { Rockstuhl, Ang, } \\
\text { Ng, Lievens, \& Van } \\
\text { Dyne }(2015)\end{array}$ & Study 3 of 2 & $\begin{array}{l}\text { replication or } \\
\text { replicate }\end{array}$ & dependent & $\begin{array}{l}\text { dependent incremental } \\
\text { constructive replication }\end{array}$ \\
\hline 353 & JAP & $\begin{array}{l}\text { abstract } \\
\& \text { title }\end{array}$ & $\begin{array}{l}\text { Rockstuhl, Ang, } \\
\text { Ng, Lievens, \& Van } \\
\text { Dyne (2015) }\end{array}$ & Study 4 of 2 & $\begin{array}{l}\text { replication or } \\
\text { replicate }\end{array}$ & dependent & $\begin{array}{l}\text { dependent incremental } \\
\text { constructive replication }\end{array}$ \\
\hline 354 & JAP & $\begin{array}{l}\text { abstract } \\
\& \text { title }\end{array}$ & $\begin{array}{l}\text { Rockstuhl, Ang, } \\
\text { Ng, Lievens, \& Van } \\
\text { Dyne (2015) }\end{array}$ & Study 2 & $\begin{array}{l}\text { replication or } \\
\text { replicate }\end{array}$ & dependent & $\begin{array}{l}\text { dependent quasi-random } \\
\text { reproducibility }\end{array}$ \\
\hline 355 & JAP & full text & $\begin{array}{l}\text { Ryan \& Nguyen } \\
(2017)\end{array}$ & $\begin{array}{l}\text { previous } \\
\text { research }\end{array}$ & $\begin{array}{l}\text { replication or } \\
\text { replicate }\end{array}$ & independent & $\begin{array}{l}\text { independent quasi random } \\
\text { reproducibility }\end{array}$ \\
\hline 356 & JAP & full text & $\begin{array}{l}\text { Schaumberg \& } \\
\text { Flynn (2017) }\end{array}$ & $\begin{array}{l}\text { study } 2 \text { of study } \\
1\end{array}$ & $\begin{array}{l}\text { replication or } \\
\text { replicate }\end{array}$ & dependent & $\begin{array}{l}\text { dependent confounded } \\
\text { replication }\end{array}$ \\
\hline 357 & JAP & full text & $\begin{array}{l}\text { Schleicher, Smith, } \\
\text { Casper, Watt, \& } \\
\text { Greguras (2015) }\end{array}$ & $\begin{array}{l}\text { previous } \\
\text { research }\end{array}$ & $\begin{array}{l}\text { replication or } \\
\text { replicate }\end{array}$ & dependent & $\begin{array}{l}\text { dependent quasi-random } \\
\text { reproducibility }\end{array}$ \\
\hline
\end{tabular}




\begin{tabular}{|c|c|c|c|c|c|c|c|}
\hline \# & Journal & Search & Study & $\begin{array}{l}\text { Which } \\
\text { repetition }\end{array}$ & Terminology & Independence & $\begin{array}{l}\text { Type of replication or } \\
\text { reproducibility study }\end{array}$ \\
\hline 358 & JAP & $\begin{array}{l}\text { abstract } \\
\& \text { title }\end{array}$ & $\begin{array}{l}\text { Schultze, Pfeiffer, } \\
\text { \& Schulz-Hardt } \\
\text { (2012) }\end{array}$ & $\begin{array}{l}\text { experiment } 2 \text { of } \\
\text { experiment } 1\end{array}$ & $\begin{array}{l}\text { replication or } \\
\text { replicate }\end{array}$ & dependent & dependent literal replication \\
\hline 359 & JAP & $\begin{array}{l}\text { abstract } \\
\& \text { title }\end{array}$ & $\begin{array}{l}\text { Schultze, Pfeiffer, } \\
\text { \& Schulz-Hardt } \\
(2012)\end{array}$ & $\begin{array}{l}\text { experiment } 4 \text { of } \\
\text { experiments } 1-3\end{array}$ & $\begin{array}{l}\text { replication or } \\
\text { replicate }\end{array}$ & dependent & $\begin{array}{l}\text { dependent quasi-random } \\
\text { replication }\end{array}$ \\
\hline 360 & JAP & $\begin{array}{l}\text { abstract } \\
\& \text { title }\end{array}$ & $\begin{array}{l}\text { Schultze, Pfeiffer, } \\
\text { \& Schulz-Hardt } \\
(2012)\end{array}$ & $\begin{array}{l}\text { experiment } 3 \text { of } \\
\text { experiments } 1 \\
\text { and } 2\end{array}$ & $\begin{array}{l}\text { replication or } \\
\text { replicate }\end{array}$ & dependent & $\begin{array}{l}\text { dependent quasi-random } \\
\text { replication }\end{array}$ \\
\hline 361 & JAP & $\begin{array}{l}\text { abstract } \\
\& \text { title }\end{array}$ & $\begin{array}{l}\text { Schultze, Pfeiffer, } \\
\& \text { Schulz-Hardt } \\
(2012)\end{array}$ & $\begin{array}{l}\text { experiment } 1 \text { of } \\
\text { previous } \\
\text { research }\end{array}$ & $\begin{array}{l}\text { direct } \\
\text { replication }\end{array}$ & independent & $\begin{array}{l}\text { independent literal } \\
\text { replication }\end{array}$ \\
\hline 362 & JAP & full text & $\begin{array}{l}\text { Scott, Restubog, \& } \\
\text { Zagenczyk (2013) }\end{array}$ & $\begin{array}{l}\text { study } 2 \text { of study } \\
1\end{array}$ & $\begin{array}{l}\text { replication or } \\
\text { replicate }\end{array}$ & dependent & $\begin{array}{l}\text { dependent incremental } \\
\text { constructive replication }\end{array}$ \\
\hline 363 & JAP & full text & $\begin{array}{l}\text { Sheldon, Dunning, } \\
\text { \& Ames (2014) }\end{array}$ & $\begin{array}{l}\text { study } 3 \text { of study } \\
1 \text { and } 2\end{array}$ & $\begin{array}{l}\text { replication or } \\
\text { replicate }\end{array}$ & dependent & $\begin{array}{l}\text { dependent quasi-random } \\
\text { replication }\end{array}$ \\
\hline 364 & JAP & full text & $\begin{array}{l}\text { Sheldon, Dunning, } \\
\text { \& Ames (2014) }\end{array}$ & study 2 & $\begin{array}{l}\text { replication or } \\
\text { replicate }\end{array}$ & dependent & $\begin{array}{l}\text { dependent quasi-random } \\
\text { replication }\end{array}$ \\
\hline 365 & JAP & full text & $\begin{array}{l}\text { Sheldon, Dunning, } \\
\text { \& Ames (2014) }\end{array}$ & $\begin{array}{l}\text { previous } \\
\text { research }\end{array}$ & $\begin{array}{l}\text { replication or } \\
\text { replicate }\end{array}$ & independent & $\begin{array}{l}\text { independent quasi-random } \\
\text { replication }\end{array}$ \\
\hline
\end{tabular}




\begin{tabular}{|c|c|c|c|c|c|c|c|}
\hline \# & Journal & Search & Study & $\begin{array}{l}\text { Which } \\
\text { repetition }\end{array}$ & Terminology & Independence & $\begin{array}{l}\text { Type of replication or } \\
\text { reproducibility study }\end{array}$ \\
\hline 366 & JAP & full text & $\begin{array}{l}\text { Shoss, Eisenberger, } \\
\text { Restubog, \& } \\
\text { Zagenczyk (2013) }\end{array}$ & $\begin{array}{l}\text { study } 3 \text { of study } \\
1 \text { and } 2\end{array}$ & $\begin{array}{l}\text { constructive } \\
\text { replication }\end{array}$ & dependent & $\begin{array}{l}\text { dependent incremental } \\
\text { constructive replication }\end{array}$ \\
\hline 367 & JAP & full text & $\begin{array}{l}\text { Shoss, Eisenberger, } \\
\text { Restubog, \& } \\
\text { Zagenczyk (2013) }\end{array}$ & $\begin{array}{l}\text { study } 2 \text { of study } \\
1\end{array}$ & $\begin{array}{l}\text { constructive } \\
\text { replication }\end{array}$ & dependent & $\begin{array}{l}\text { dependent regressive } \\
\text { replication }\end{array}$ \\
\hline 368 & JAP & $\begin{array}{l}\text { abstract } \\
\& \text { title }\end{array}$ & $\begin{array}{l}\text { Sinaceur, Van } \\
\text { Kleef, Neale, } \\
\text { Adam, \& Haag } \\
\text { (2011) }\end{array}$ & $\begin{array}{l}\text { experiment } 3 \text { of } \\
\text { experiment } 2\end{array}$ & $\begin{array}{l}\text { replication or } \\
\text { replicate }\end{array}$ & dependent & $\begin{array}{l}\text { dependent incremental } \\
\text { constructive replication }\end{array}$ \\
\hline 369 & JAP & $\begin{array}{l}\text { abstract } \\
\& \text { title }\end{array}$ & $\begin{array}{l}\text { Sinaceur, Van } \\
\text { Kleef, Neale, } \\
\text { Adam, \& Haag } \\
\text { (2011) }\end{array}$ & $\begin{array}{l}\text { experiment } 1 \text { of } \\
\text { previous } \\
\text { research }\end{array}$ & $\begin{array}{l}\text { replication or } \\
\text { replicate }\end{array}$ & & first time hypothesis test \\
\hline 370 & JAP & full text & $\begin{array}{l}\text { Sinha, Janardhanan, } \\
\text { Greer, Conlon, \& } \\
\text { Edwards (2016) }\end{array}$ & $\begin{array}{l}\text { study } 2 \text { of study } \\
1\end{array}$ & $\begin{array}{l}\text { replication or } \\
\text { replicate }\end{array}$ & dependent & $\begin{array}{l}\text { dependent substantial } \\
\text { constructive replication }\end{array}$ \\
\hline 371 & JAP & $\begin{array}{l}\text { abstract } \\
\& \text { title }\end{array}$ & $\begin{array}{l}\text { Skarlicki, van } \\
\text { Jaarsveld, Shao, } \\
\text { Song, \& Wang } \\
\text { (2016) }\end{array}$ & Study 2 of 1 & $\begin{array}{l}\text { replication or } \\
\text { replicate }\end{array}$ & dependent & $\begin{array}{l}\text { dependent quasi-random } \\
\text { reproducibility }\end{array}$ \\
\hline
\end{tabular}




\begin{tabular}{|c|c|c|c|c|c|c|c|}
\hline \# & Journal & Search & Study & $\begin{array}{l}\text { Which } \\
\text { repetition }\end{array}$ & Terminology & Independence & $\begin{array}{l}\text { Type of replication or } \\
\text { reproducibility study }\end{array}$ \\
\hline 372 & JAP & $\begin{array}{l}\text { abstract } \\
\& \text { title }\end{array}$ & $\begin{array}{l}\text { Skarlicki, van } \\
\text { Jaarsveld, Shao, } \\
\text { Song, \& Wang } \\
\text { (2016) }\end{array}$ & $\begin{array}{l}\text { Study } 1 \text { of other } \\
\text { parts of study } 1\end{array}$ & $\begin{array}{l}\text { replication or } \\
\text { replicate }\end{array}$ & dependent & $\begin{array}{l}\text { dependent quasi-random } \\
\text { reproducibility }\end{array}$ \\
\hline 373 & JAP & $\begin{array}{l}\text { abstract } \\
\& \text { title }\end{array}$ & $\begin{array}{l}\text { Sliter, Sinclair, } \\
\text { Yuan, \& Mohr } \\
\text { (2014) }\end{array}$ & Study 2 of 1 & $\begin{array}{l}\text { replication or } \\
\text { replicate }\end{array}$ & dependent & $\begin{array}{l}\text { dependent substantial } \\
\text { constructive replication }\end{array}$ \\
\hline 374 & JAP & full text & $\begin{array}{l}\text { Sonnentag, Mojza, } \\
\text { Demerouti, \& } \\
\text { Bakker (2012) }\end{array}$ & $\begin{array}{l}\text { previous } \\
\text { research }\end{array}$ & $\begin{array}{l}\text { replication or } \\
\text { replicate }\end{array}$ & $\begin{array}{l}\text { semi- } \\
\text { independent }\end{array}$ & $\begin{array}{l}\text { semi-independent quasi- } \\
\text { random replication }\end{array}$ \\
\hline 375 & JAP & full text & $\begin{array}{l}\text { Speer, Christiansen, } \\
\text { Goffin, \& Goff } \\
(2014)\end{array}$ & $\begin{array}{l}\text { sample } 2 \text { of } \\
\text { previous } \\
\text { research }\end{array}$ & $\begin{array}{l}\text { replication or } \\
\text { replicate }\end{array}$ & dependent & $\begin{array}{l}\text { dependent quasi-random } \\
\text { replication }\end{array}$ \\
\hline 376 & JAP & full text & $\begin{array}{l}\text { Speer, Christiansen, } \\
\text { Goffin, \& Goff } \\
(2014)\end{array}$ & $\begin{array}{l}\text { sample } 1 \text { of } \\
\text { previous } \\
\text { research }\end{array}$ & $\begin{array}{l}\text { replication or } \\
\text { replicate }\end{array}$ & independent & $\begin{array}{l}\text { independent quasi-random } \\
\text { replication }\end{array}$ \\
\hline 377 & JAP & full text & $\begin{array}{l}\text { Speer, Christiansen, } \\
\text { Goffin, \& Goff } \\
(2014)\end{array}$ & $\begin{array}{l}\text { sample } 3 \text { of } \\
\text { previous } \\
\text { research }\end{array}$ & $\begin{array}{l}\text { replication or } \\
\text { replicate }\end{array}$ & independent & $\begin{array}{l}\text { independent quasi-random } \\
\text { replication }\end{array}$ \\
\hline 378 & JAP & full text & $\begin{array}{l}\text { Spieler, Scheibe, } \\
\text { Stamov-Ronagel, \& } \\
\text { Kappas (2017) }\end{array}$ & $\begin{array}{l}\text { study } 2 \text { of study } \\
1\end{array}$ & $\begin{array}{l}\text { replication or } \\
\text { replicate }\end{array}$ & dependent & $\begin{array}{l}\text { dependent incremental } \\
\text { constructive replication }\end{array}$ \\
\hline
\end{tabular}




\begin{tabular}{|c|c|c|c|c|c|c|c|}
\hline \# & Journal & Search & Study & $\begin{array}{l}\text { Which } \\
\text { repetition }\end{array}$ & Terminology & Independence & $\begin{array}{l}\text { Type of replication or } \\
\text { reproducibility study }\end{array}$ \\
\hline 379 & JAP & full text & $\begin{array}{l}\text { Spieler, Scheibe, } \\
\text { Stamov-Ronagel, \& } \\
\text { Kappas (2017) }\end{array}$ & $\begin{array}{l}\text { study } 1 \text { of } \\
\text { previous } \\
\text { research }\end{array}$ & $\begin{array}{l}\text { replication or } \\
\text { replicate }\end{array}$ & independent & $\begin{array}{l}\text { independent quasi-random } \\
\text { replication }\end{array}$ \\
\hline 380 & JAP & full text & $\begin{array}{l}\text { Stoverink, } \\
\text { Umphress, Gardner, } \\
\text { \& Miner (2014) }\end{array}$ & $\begin{array}{l}\text { study } 2 \text { of study } \\
1\end{array}$ & $\begin{array}{l}\text { replication or } \\
\text { replicate }\end{array}$ & dependent & $\begin{array}{l}\text { dependent quasi-random } \\
\text { replication }\end{array}$ \\
\hline 381 & JAP & full text & $\begin{array}{l}\text { Strauss, Griffin, \& } \\
\text { Parker (2012) }\end{array}$ & $\begin{array}{l}\text { study } 2 \text { of } \\
\text { studies } 1\end{array}$ & nothing & dependent & $\begin{array}{l}\text { dependent incremental } \\
\text { constructive replication }\end{array}$ \\
\hline 382 & JAP & full text & $\begin{array}{l}\text { Strauss, Griffin, \& } \\
\text { Parker (2012) }\end{array}$ & study $1 \mathrm{~b}$ of $1 \mathrm{a}$ & $\begin{array}{l}\text { replication or } \\
\text { replicate }\end{array}$ & dependent & $\begin{array}{l}\text { dependent regressive } \\
\text { replication }\end{array}$ \\
\hline 383 & JAP & full text & $\begin{array}{l}\text { Strauss, Griffin, \& } \\
\text { Parker (2012) }\end{array}$ & study 3 & nothing & dependent & generalizability \\
\hline 384 & JAP & full text & $\begin{array}{l}\text { Sturman, Shao, \& } \\
\text { Katz (2012) }\end{array}$ & & $\begin{array}{l}\text { replication or } \\
\text { replicate }\end{array}$ & dependent & $\begin{array}{l}\text { dependent quasi-random } \\
\text { reproducibility }\end{array}$ \\
\hline 385 & JAP & full text & $\begin{array}{l}\text { Sturman, Shao, \& } \\
\text { Katz (2012) }\end{array}$ & $\begin{array}{l}\text { previous } \\
\text { research }\end{array}$ & $\begin{array}{l}\text { replication or } \\
\text { replicate }\end{array}$ & independent & $\begin{array}{l}\text { independent quasi-random } \\
\text { replication }\end{array}$ \\
\hline 386 & JAP & full text & $\begin{array}{l}\text { Sun, Song, \& Lim } \\
(2013)\end{array}$ & & $\begin{array}{l}\text { replication or } \\
\text { replicate }\end{array}$ & dependent & $\begin{array}{l}\text { dependent quasi-random } \\
\text { reproducibility }\end{array}$ \\
\hline 387 & JAP & full text & $\begin{array}{l}\text { Sun, Song, \& Lim } \\
(2013)\end{array}$ & $\begin{array}{l}\text { previous } \\
\text { research }\end{array}$ & $\begin{array}{l}\text { replication or } \\
\text { replicate }\end{array}$ & independent & $\begin{array}{l}\text { independent quasi-random } \\
\text { replication }\end{array}$ \\
\hline
\end{tabular}




\begin{tabular}{|c|c|c|c|c|c|c|c|}
\hline \# & Journal & Search & Study & $\begin{array}{l}\text { Which } \\
\text { repetition }\end{array}$ & Terminology & Independence & $\begin{array}{l}\text { Type of replication or } \\
\text { reproducibility study }\end{array}$ \\
\hline 388 & JAP & $\begin{array}{l}\text { abstract } \\
\& \text { title }\end{array}$ & $\begin{array}{l}\text { Thau, Derfler- } \\
\text { Rozin, Pitesa, } \\
\text { Mitchell, \& Pillutla } \\
2015\end{array}$ & Study 2 of 1 & $\begin{array}{l}\text { constructive } \\
\text { replication }\end{array}$ & dependent & $\begin{array}{l}\text { dependent confounded } \\
\text { replication }\end{array}$ \\
\hline 389 & JAP & full text & $\begin{array}{l}\text { Unsworth \& } \\
\text { McNeill (2017) }\end{array}$ & $\begin{array}{l}\text { study } 2 \text { of study } \\
1\end{array}$ & nothing & dependent & $\begin{array}{l}\text { dependent confounded } \\
\text { replication }\end{array}$ \\
\hline 390 & JAP & full text & $\begin{array}{l}\text { Unsworth \& } \\
\text { McNeill (2017) }\end{array}$ & $\begin{array}{l}\text { study } 3 \text { of study } \\
2\end{array}$ & $\begin{array}{l}\text { replication or } \\
\text { replicate }\end{array}$ & dependent & $\begin{array}{l}\text { dependent incremental } \\
\text { constructive replication }\end{array}$ \\
\hline 391 & JAP & full text & $\begin{array}{l}\text { van Hooft \& Born } \\
(2012)\end{array}$ & $\begin{array}{l}\text { previous } \\
\text { research }\end{array}$ & $\begin{array}{l}\text { replication or } \\
\text { replicate }\end{array}$ & independent & $\begin{array}{l}\text { independent quasi-random } \\
\text { replication }\end{array}$ \\
\hline 392 & JAP & full text & $\begin{array}{l}\text { Van Iddekinge, } \\
\text { Roth, Raymark, \& } \\
\text { Odle-Dusseau } \\
(2012)\end{array}$ & $\begin{array}{l}\text { previous } \\
\text { research }\end{array}$ & $\begin{array}{l}\text { replication or } \\
\text { replicate }\end{array}$ & independent & $\begin{array}{l}\text { independent constructive } \\
\text { reproducibility }\end{array}$ \\
\hline 393 & JAP & full text & $\begin{array}{l}\text { Van Kleef, Steinel, } \\
\text { \& Homan (2013) }\end{array}$ & $\begin{array}{l}\text { experiment } 2 \text { of } \\
1\end{array}$ & $\begin{array}{l}\text { replication or } \\
\text { replicate }\end{array}$ & dependent & $\begin{array}{l}\text { dependent incremental } \\
\text { constructive replication }\end{array}$ \\
\hline 394 & JAP & full text & $\begin{array}{l}\text { Van Kleef, van den } \\
\text { Berg, \& Heerdink } \\
(2015)\end{array}$ & experiment 4 & nothing & dependent & $\begin{array}{l}\text { dependent substantial } \\
\text { constructive replication }\end{array}$ \\
\hline
\end{tabular}




\begin{tabular}{|c|c|c|c|c|c|c|c|}
\hline \# & Journal & Search & Study & $\begin{array}{l}\text { Which } \\
\text { repetition }\end{array}$ & Terminology & Independence & $\begin{array}{l}\text { Type of replication or } \\
\text { reproducibility study }\end{array}$ \\
\hline 395 & JAP & full text & $\begin{array}{l}\text { Van Kleef, van den } \\
\text { Berg, \& Heerdink } \\
(2015)\end{array}$ & $\begin{array}{l}\text { experiment } 2 \text { of } \\
\text { experiment } 1\end{array}$ & $\begin{array}{l}\text { replication or } \\
\text { replicate }\end{array}$ & dependent & $\begin{array}{l}\text { dependent incremental } \\
\text { constructive replication }\end{array}$ \\
\hline 396 & JAP & full text & $\begin{array}{l}\text { Van Kleef, van den } \\
\text { Berg, \& Heerdink } \\
(2015)\end{array}$ & $\begin{array}{l}\text { experiment } 3 \text { of } \\
\text { experiments } 1 \\
\& 2\end{array}$ & $\begin{array}{l}\text { replicate and } \\
\text { extend }\end{array}$ & dependent & $\begin{array}{l}\text { dependent quasi-random } \\
\text { replication }\end{array}$ \\
\hline 397 & JAP & full text & $\begin{array}{l}\text { Venkataramani, } \\
\text { Labianca, \& } \\
\text { Grosser }(2013)\end{array}$ & $\begin{array}{l}\text { replication } \\
\text { study of } \\
\text { primary study }\end{array}$ & $\begin{array}{l}\text { replication or } \\
\text { replicate }\end{array}$ & dependent & $\begin{array}{l}\text { dependent quasi-random } \\
\text { replication }\end{array}$ \\
\hline 398 & JAP & full text & $\begin{array}{l}\text { Venkataramani, } \\
\text { Richter, \& Clarke } \\
(2014)\end{array}$ & & $\begin{array}{l}\text { replication or } \\
\text { replicate }\end{array}$ & dependent & $\begin{array}{l}\text { dependent quasi-random } \\
\text { reproducibility }\end{array}$ \\
\hline 399 & JAP & full text & $\begin{array}{l}\text { Walker, van } \\
\text { Jaarsveld, \& } \\
\text { Skarlicki (2017) }\end{array}$ & & $\begin{array}{l}\text { replication or } \\
\text { replicate }\end{array}$ & dependent & $\begin{array}{l}\text { dependent constructive } \\
\text { reproducibility }\end{array}$ \\
\hline 400 & JAP & full text & $\begin{array}{l}\text { Walker, Feild, } \\
\text { Bernerth, \& Becton } \\
(2012)\end{array}$ & $\begin{array}{l}\text { study } 2 \text { of } \\
\text { study } 1\end{array}$ & $\begin{array}{l}\text { replication or } \\
\text { replicate }\end{array}$ & dependent & $\begin{array}{l}\text { dependent confounded } \\
\text { replication }\end{array}$ \\
\hline 401 & JAP & full text & $\begin{array}{l}\text { Walter, Lam, van } \\
\text { der Vegt, Huang, \& } \\
\text { Miao (2015) }\end{array}$ & $\begin{array}{l}\text { study } 2 \text { of study } \\
1\end{array}$ & $\begin{array}{l}\text { constructive } \\
\text { replication }\end{array}$ & dependent & $\begin{array}{l}\text { dependent confounded } \\
\text { replication }\end{array}$ \\
\hline
\end{tabular}




\begin{tabular}{|c|c|c|c|c|c|c|c|}
\hline \# & Journal & Search & Study & $\begin{array}{l}\text { Which } \\
\text { repetition }\end{array}$ & Terminology & Independence & $\begin{array}{l}\text { Type of replication or } \\
\text { reproducibility study }\end{array}$ \\
\hline 402 & JAP & full text & $\begin{array}{l}\text { Wang \& } \\
\text { Murnighan (2017) }\end{array}$ & $\begin{array}{l}\text { study } 2 \text { of study } \\
1\end{array}$ & nothing & dependent & $\begin{array}{l}\text { dependent quasi-random } \\
\text { replication }\end{array}$ \\
\hline 403 & JAP & full text & $\begin{array}{l}\text { Wang \& } \\
\text { Murnighan (2017) }\end{array}$ & $\begin{array}{l}\text { study } 3 \text { of } \\
\text { studies } 1 \text { and } 2\end{array}$ & $\begin{array}{l}\text { behavioral } \\
\text { replication }\end{array}$ & dependent & $\begin{array}{l}\text { dependent substantial } \\
\text { constructive replication }\end{array}$ \\
\hline 404 & JAP & full text & $\begin{array}{l}\text { Wang, Lu, \& Siu } \\
(2015)\end{array}$ & $\begin{array}{l}\text { study } 2 \text { of study } \\
1\end{array}$ & $\begin{array}{l}\text { replication or } \\
\text { replicate }\end{array}$ & dependent & $\begin{array}{l}\text { dependent quasi-random } \\
\text { replication }\end{array}$ \\
\hline 405 & JAP & full text & $\begin{array}{l}\text { Wang, Liu, Liao, } \\
\text { Gong, Kammeyer- } \\
\text { Mueller, \& Shi } \\
\text { (2013). }\end{array}$ & & $\begin{array}{l}\text { replication or } \\
\text { replicate }\end{array}$ & dependent & $\begin{array}{l}\text { dependent quasi-random } \\
\text { reproducibility }\end{array}$ \\
\hline 406 & JAP & full text & $\begin{array}{l}\text { Wang, Waldman, \& } \\
\text { Zhang (2014) }\end{array}$ & & $\begin{array}{l}\text { replication or } \\
\text { replicate }\end{array}$ & dependent & $\begin{array}{l}\text { dependent quasi-random } \\
\text { reproducibility }\end{array}$ \\
\hline 407 & JAP & full text & $\begin{array}{l}\text { Ward, Ravlin, } \\
\text { Klaas, Ployhart, \& } \\
\text { Buchan (2016) }\end{array}$ & $\begin{array}{l}\text { study } 2 \text { of study } \\
1\end{array}$ & $\begin{array}{l}\text { replication or } \\
\text { replicate }\end{array}$ & dependent & $\begin{array}{l}\text { dependent confounded } \\
\text { replication }\end{array}$ \\
\hline 408 & JAP & $\begin{array}{l}\text { abstract } \\
\& \text { title }\end{array}$ & $\begin{array}{l}\text { Wegge, Roth, } \\
\text { Neubach, Schmidt, } \\
\text { \& Kanfer (2008) }\end{array}$ & Study 2 of 1 & $\begin{array}{l}\text { replication or } \\
\text { replicate }\end{array}$ & dependent & dependent literal replication \\
\hline 409 & JAP & $\begin{array}{l}\text { abstract } \\
\& \text { title }\end{array}$ & $\begin{array}{l}\text { Wellman, Mayer, } \\
\text { Ong, \& DeRue } \\
(2016)\end{array}$ & $\begin{array}{l}\text { Study } 3 \text { of } \\
\text { Study } 2\end{array}$ & $\begin{array}{l}\text { replicate and } \\
\text { extend }\end{array}$ & dependent & $\begin{array}{l}\text { dependent confounded } \\
\text { replication }\end{array}$ \\
\hline
\end{tabular}




\begin{tabular}{|c|c|c|c|c|c|c|c|}
\hline \# & Journal & Search & Study & $\begin{array}{l}\text { Which } \\
\text { repetition }\end{array}$ & Terminology & Independence & $\begin{array}{l}\text { Type of replication or } \\
\text { reproducibility study }\end{array}$ \\
\hline 410 & JAP & full text & $\begin{array}{l}\text { Whiting, Maynes, } \\
\text { Podsakoff, \& } \\
\text { Podsakoff (2012) }\end{array}$ & $\begin{array}{l}\text { study } 3 \text { of } \\
\text { studies } 1 \text { and } 2\end{array}$ & $\begin{array}{l}\text { replication or } \\
\text { replicate }\end{array}$ & dependent & dependent literal replication \\
\hline 411 & JAP & full text & $\begin{array}{l}\text { Williams \& } \\
\text { O'Boyle (2015) }\end{array}$ & $\begin{array}{l}\text { previous } \\
\text { research }\end{array}$ & $\begin{array}{l}\text { replication or } \\
\text { replicate }\end{array}$ & independent & $\begin{array}{l}\text { independent literal } \\
\text { replication }\end{array}$ \\
\hline 412 & JAP & full text & $\begin{array}{l}\text { Winterich, Aquino, } \\
\text { Mittal, \& Swartz } \\
\text { (2013) }\end{array}$ & $\begin{array}{l}\text { study } 2 \text { of study } \\
1\end{array}$ & $\begin{array}{l}\text { constructive } \\
\text { replication }\end{array}$ & dependent & $\begin{array}{l}\text { dependent quasi-random } \\
\text { replication }\end{array}$ \\
\hline 413 & JAP & $\begin{array}{l}\text { abstract } \\
\& \text { title }\end{array}$ & $\begin{array}{l}\text { Wu, Liu, Kwan, \& } \\
\text { Lee (2016) }\end{array}$ & Study 2 of 1 & $\begin{array}{l}\text { replication or } \\
\text { replicate }\end{array}$ & dependent & $\begin{array}{l}\text { dependent incremental } \\
\text { constructive replication }\end{array}$ \\
\hline 414 & JAP & full text & $\begin{array}{l}\text { Wu \& Griffin } \\
(2012)\end{array}$ & $\begin{array}{l}\text { previous } \\
\text { research }\end{array}$ & $\begin{array}{l}\text { replication or } \\
\text { replicate }\end{array}$ & independent & $\begin{array}{l}\text { independent confounded } \\
\text { replication }\end{array}$ \\
\hline 415 & JAP & full text & $\begin{array}{l}\text { Yam, Fehr, \& } \\
\text { Barnes (2014) }\end{array}$ & $\begin{array}{l}\text { study } 3 \text { of } \\
\text { studies } 1 \text { and } 2\end{array}$ & $\begin{array}{l}\text { constructive } \\
\text { replication }\end{array}$ & dependent & $\begin{array}{l}\text { dependent confounded } \\
\text { replication }\end{array}$ \\
\hline 416 & JAP & full text & $\begin{array}{l}\text { Yu, Downes, } \\
\text { Carter, \& O'Boyle } \\
(2016)\end{array}$ & $\begin{array}{l}\text { previous } \\
\text { research }\end{array}$ & $\begin{array}{l}\text { replication or } \\
\text { replicate }\end{array}$ & independent & $\begin{array}{l}\text { independent literal } \\
\text { reproducibility }\end{array}$ \\
\hline 417 & JAP & full text & $\begin{array}{l}\text { Zerres, Huffmeier, } \\
\text { Freund, Backhaus, } \\
\& \text { Hertel (2013) }\end{array}$ & $\begin{array}{l}\text { previous } \\
\text { research }\end{array}$ & $\begin{array}{l}\text { replication or } \\
\text { replicate }\end{array}$ & independent & $\begin{array}{l}\text { independent quasi-random } \\
\text { replication }\end{array}$ \\
\hline
\end{tabular}




\begin{tabular}{|c|c|c|c|c|c|c|c|}
\hline \# & Journal & Search & Study & $\begin{array}{l}\text { Which } \\
\text { repetition }\end{array}$ & Terminology & Independence & $\begin{array}{l}\text { Type of replication or } \\
\text { reproducibility study }\end{array}$ \\
\hline 418 & JAP & full text & $\begin{array}{l}\text { Zhan, Wang, \& Shi } \\
(2015)\end{array}$ & $\begin{array}{l}\text { previous } \\
\text { research }\end{array}$ & $\begin{array}{l}\text { replication or } \\
\text { replicate }\end{array}$ & independent & $\begin{array}{l}\text { independent quasi-random } \\
\text { replication }\end{array}$ \\
\hline 419 & JAP & full text & $\begin{array}{l}\text { Zhou, Wang, Chen, } \\
\text { \& Shi (2012) }\end{array}$ & $\begin{array}{l}\text { previous } \\
\text { research }\end{array}$ & $\begin{array}{l}\text { constructive } \\
\text { replication }\end{array}$ & independent & $\begin{array}{l}\text { independent incremental } \\
\text { constructive replication }\end{array}$ \\
\hline 420 & JAP & full text & $\begin{array}{l}\text { Zhu, Aquino, \& } \\
\text { Vadera (2016) }\end{array}$ & $\begin{array}{l}\text { study } 2 \text { of study } \\
1\end{array}$ & $\begin{array}{l}\text { replication or } \\
\text { replicate }\end{array}$ & dependent & $\begin{array}{l}\text { dependent quasi-random } \\
\text { replication }\end{array}$ \\
\hline 421 & JAP & full text & $\begin{array}{l}\text { Zhu, Aquino, \& } \\
\text { Vadera (2016) }\end{array}$ & $\begin{array}{l}\text { study } 5 \text { of study } \\
1\end{array}$ & $\begin{array}{l}\text { replication or } \\
\text { replicate }\end{array}$ & dependent & $\begin{array}{l}\text { dependent quasi-random } \\
\text { replication }\end{array}$ \\
\hline 422 & JAP & full text & $\begin{array}{l}\text { Zhu, Aquino, \& } \\
\text { Vadera (2016) }\end{array}$ & $\begin{array}{l}\text { study } 3 \text { of study } \\
1\end{array}$ & $\begin{array}{l}\text { replication or } \\
\text { replicate }\end{array}$ & dependent & $\begin{array}{l}\text { dependent substantial } \\
\text { constructive replication }\end{array}$ \\
\hline 423 & JAP & full text & $\begin{array}{l}\text { Zhu, Aquino, \& } \\
\text { Vadera (2016) }\end{array}$ & study 4 of study & $\begin{array}{l}\text { constructive } \\
\text { replication }\end{array}$ & dependent & $\begin{array}{l}\text { dependent quasi-random } \\
\text { replication }\end{array}$ \\
\hline 424 & $\mathrm{JOM}$ & full text & $\begin{array}{l}\text { Abbas, Raja, Darr, } \\
\text { \& Bouckenooghe } \\
(2014)\end{array}$ & $\begin{array}{l}\text { previous } \\
\text { research }\end{array}$ & $\begin{array}{l}\text { replication or } \\
\text { replicate }\end{array}$ & independent & generalizability \\
\hline 425 & JOM & full text & $\begin{array}{l}\text { Aryee, Walumbwa, } \\
\text { Mondejar, \& Chu } \\
(2017)\end{array}$ & $\begin{array}{l}\text { previous } \\
\text { research }\end{array}$ & $\begin{array}{l}\text { replication or } \\
\text { replicate }\end{array}$ & independent & generalizability \\
\hline 426 & JOM & full text & Bagger \& Li (2014) & $\begin{array}{l}\text { previous } \\
\text { research }\end{array}$ & $\begin{array}{l}\text { replication or } \\
\text { replicate }\end{array}$ & independent & $\begin{array}{l}\text { independent quasi-random } \\
\text { replication }\end{array}$ \\
\hline
\end{tabular}




\begin{tabular}{|c|c|c|c|c|c|c|c|}
\hline \# & Journal & Search & Study & $\begin{array}{l}\text { Which } \\
\text { repetition }\end{array}$ & Terminology & Independence & $\begin{array}{l}\text { Type of replication or } \\
\text { reproducibility study }\end{array}$ \\
\hline 427 & $\mathrm{JOM}$ & full text & $\begin{array}{l}\text { Bardolet, Brown, \& } \\
\text { Lovallo (2017) }\end{array}$ & $\begin{array}{l}\text { previous } \\
\text { research }\end{array}$ & $\begin{array}{l}\text { replication or } \\
\text { replicate }\end{array}$ & $\begin{array}{l}\text { semi- } \\
\text { independent }\end{array}$ & $\begin{array}{l}\text { semi-independent quasi- } \\
\text { random replication }\end{array}$ \\
\hline 428 & $\mathrm{JOM}$ & full text & $\begin{array}{l}\text { Belschak, Hartog, } \\
\text { \& Kalshoven } \\
(2015)\end{array}$ & $\begin{array}{l}\text { study } 2 \text { of study } \\
1\end{array}$ & $\begin{array}{l}\text { replication or } \\
\text { replicate }\end{array}$ & dependent & $\begin{array}{l}\text { dependent quasi-random } \\
\text { replication }\end{array}$ \\
\hline 429 & $\mathrm{JOM}$ & $\begin{array}{l}\text { abstract } \\
\& \text { title }\end{array}$ & $\begin{array}{l}\text { Beus \& Whitman } \\
(2017)\end{array}$ & $\begin{array}{l}\text { sample } 2 \text { of } \\
\text { sample } 1\end{array}$ & $\begin{array}{l}\text { replication or } \\
\text { replicate }\end{array}$ & dependent & $\begin{array}{l}\text { dependent quasi-random } \\
\text { replication }\end{array}$ \\
\hline 430 & $\mathrm{JOM}$ & full text & Bizzi (2013) & study 1 & $\begin{array}{l}\text { replication or } \\
\text { replicate }\end{array}$ & dependent & $\begin{array}{l}\text { dependent quasi-random } \\
\text { reproducibility }\end{array}$ \\
\hline 431 & $\mathrm{JOM}$ & full text & $\begin{array}{l}\text { Bordia, Restubog, } \\
\text { Bordia, \& Tang } \\
(2017)\end{array}$ & $\begin{array}{l}\text { sample } 2 \text { of } \\
\text { sample } 1\end{array}$ & $\begin{array}{l}\text { constructive } \\
\text { replication }\end{array}$ & dependent & $\begin{array}{l}\text { dependent quasi-random } \\
\text { replication }\end{array}$ \\
\hline 432 & $\mathrm{JOM}$ & full text & $\begin{array}{l}\text { Bordia, Restubog, } \\
\text { Bordia, \& Tang } \\
(2017)\end{array}$ & $\begin{array}{l}\text { previous } \\
\text { research }\end{array}$ & $\begin{array}{l}\text { replication or } \\
\text { replicate }\end{array}$ & independent & $\begin{array}{l}\text { independent quasi-random } \\
\text { replication }\end{array}$ \\
\hline 433 & $\mathrm{JOM}$ & full text & $\begin{array}{l}\text { Carlson, Hunter, } \\
\text { Ferguson, \& } \\
\text { Whitten (2014) }\end{array}$ & $\begin{array}{l}\text { previous } \\
\text { research }\end{array}$ & $\begin{array}{l}\text { replication or } \\
\text { replicate }\end{array}$ & independent & $\begin{array}{l}\text { independent quasi-random } \\
\text { replication }\end{array}$ \\
\hline 434 & JOM & full text & $\begin{array}{l}\text { Chiaburu, } \\
\text { Stoverink, Li, \& } \\
\text { Zhang (2015) }\end{array}$ & $\begin{array}{l}\text { study } 3 \text { of } \\
\text { studies } 1 \text { and } 2\end{array}$ & $\begin{array}{l}\text { replication or } \\
\text { replicate }\end{array}$ & dependent & $\begin{array}{l}\text { dependent quasi-random } \\
\text { replication }\end{array}$ \\
\hline
\end{tabular}




\begin{tabular}{|c|c|c|c|c|c|c|c|}
\hline \# & Journal & Search & Study & $\begin{array}{l}\text { Which } \\
\text { repetition }\end{array}$ & Terminology & Independence & $\begin{array}{l}\text { Type of replication or } \\
\text { reproducibility study }\end{array}$ \\
\hline 435 & $\mathrm{JOM}$ & full text & $\begin{array}{l}\text { Chiaburu, } \\
\text { Stoverink, Li, \& } \\
\text { Zhang (2015) }\end{array}$ & $\begin{array}{l}\text { study } 2 \text { of study } \\
1\end{array}$ & nothing & dependent & $\begin{array}{l}\text { dependent quasi-random } \\
\text { replication }\end{array}$ \\
\hline 436 & $\mathrm{JOM}$ & full text & $\begin{array}{l}\text { Collins \& } \\
\text { Mossholder (2017) }\end{array}$ & $\begin{array}{l}\text { study } 2 \text { of study } \\
1\end{array}$ & $\begin{array}{l}\text { replication or } \\
\text { replicate }\end{array}$ & dependent & $\begin{array}{l}\text { dependent confounded } \\
\text { replication }\end{array}$ \\
\hline 437 & $\mathrm{JOM}$ & full text & $\begin{array}{l}\text { Dahling, Chau, \& } \\
\text { O'Malley (2012) }\end{array}$ & $\begin{array}{l}\text { previous } \\
\text { research }\end{array}$ & $\begin{array}{l}\text { replication or } \\
\text { replicate }\end{array}$ & independent & $\begin{array}{l}\text { independent quasi-random } \\
\text { replication }\end{array}$ \\
\hline 438 & $\mathrm{JOM}$ & full text & $\begin{array}{l}\text { Daniels, Beesley, } \\
\text { Wimalasiri, \& } \\
\text { Cheyne (2013) }\end{array}$ & $\begin{array}{l}\text { previous } \\
\text { research }\end{array}$ & $\begin{array}{l}\text { replication or } \\
\text { replicate }\end{array}$ & dependent & $\begin{array}{l}\text { dependent quasi-random } \\
\text { reproducibility }\end{array}$ \\
\hline 439 & JOM & full text & $\begin{array}{l}\text { Dierdorff, Rubin, \& } \\
\text { Bachrach (2012) }\end{array}$ & $\begin{array}{l}\text { previous } \\
\text { research }\end{array}$ & $\begin{array}{l}\text { replication or } \\
\text { replicate }\end{array}$ & independent & $\begin{array}{l}\text { independent quasi-random } \\
\text { replication }\end{array}$ \\
\hline 440 & $\mathrm{JOM}$ & full text & $\begin{array}{l}\text { Dijke, Cremer, } \\
\text { Brebels, \& } \\
\text { Quaquebeke (2015) }\end{array}$ & $\begin{array}{l}\text { study } 2 \text { of study } \\
1\end{array}$ & $\begin{array}{l}\text { replication or } \\
\text { replicate }\end{array}$ & dependent & $\begin{array}{l}\text { dependent confounded } \\
\text { replication }\end{array}$ \\
\hline 441 & $\mathrm{JOM}$ & full text & $\begin{array}{l}\text { Dijke, Cremer, } \\
\text { Brebels, \& } \\
\text { Quaquebeke (2015) }\end{array}$ & $\begin{array}{l}\text { study } 3 \text { of } \\
\text { studies } 1 \text { and } 2\end{array}$ & $\begin{array}{l}\text { replication or } \\
\text { replicate }\end{array}$ & dependent & generalizability \\
\hline 442 & JOM & full text & $\begin{array}{l}\text { Dillon, Tinsley, } \\
\text { Madsen, \& Rogers } \\
\text { (2016) }\end{array}$ & $\begin{array}{l}\text { study } 4 \text { of study } \\
1\end{array}$ & $\begin{array}{l}\text { replication or } \\
\text { replicate }\end{array}$ & dependent & $\begin{array}{l}\text { dependent confounded } \\
\text { replication }\end{array}$ \\
\hline
\end{tabular}




\begin{tabular}{|c|c|c|c|c|c|c|c|}
\hline \# & Journal & Search & Study & $\begin{array}{l}\text { Which } \\
\text { repetition }\end{array}$ & Terminology & Independence & $\begin{array}{l}\text { Type of replication or } \\
\text { reproducibility study }\end{array}$ \\
\hline 443 & JOM & full text & $\begin{array}{l}\text { Dillon, Tinsley, } \\
\text { Madsen, \& Rogers } \\
\text { (2016) }\end{array}$ & $\begin{array}{l}\text { study } 3 \text { of study } \\
1\end{array}$ & $\begin{array}{l}\text { replication or } \\
\text { replicate }\end{array}$ & dependent & $\begin{array}{l}\text { dependent incremental } \\
\text { constructive replication }\end{array}$ \\
\hline 444 & JOM & full text & $\begin{array}{l}\text { Dillon, Tinsley, } \\
\text { Madsen, \& Rogers } \\
\text { (2016) }\end{array}$ & $\begin{array}{l}\text { study } 2 \text { of study } \\
1\end{array}$ & $\begin{array}{l}\text { replication or } \\
\text { replicate }\end{array}$ & dependent & $\begin{array}{l}\text { dependent confounded } \\
\text { replication }\end{array}$ \\
\hline 445 & JOM & full text & $\begin{array}{l}\text { Ferris, Spence, } \\
\text { Brown, \& Heller } \\
(2012)\end{array}$ & $\begin{array}{l}\text { previous } \\
\text { research }\end{array}$ & $\begin{array}{l}\text { replication or } \\
\text { replicate }\end{array}$ & independent & $\begin{array}{l}\text { independent quasi-random } \\
\text { replication }\end{array}$ \\
\hline 446 & JOM & full text & $\begin{array}{l}\text { González-Benito, } \\
\text { Aguinis, Boyd, \& } \\
\text { Suárez-González } \\
\text { (2012) }\end{array}$ & $\begin{array}{l}\text { previous } \\
\text { research }\end{array}$ & $\begin{array}{l}\text { replication or } \\
\text { replicate }\end{array}$ & independent & $\begin{array}{l}\text { independent quasi-random } \\
\text { replication }\end{array}$ \\
\hline 447 & JOM & full text & $\begin{array}{l}\text { Greenbaum, Hill, } \\
\text { Mawritz, \& Quade } \\
\text { (2017) }\end{array}$ & $\begin{array}{l}\text { study } 2 \text { of study } \\
1\end{array}$ & $\begin{array}{l}\text { replication or } \\
\text { replicate }\end{array}$ & dependent & $\begin{array}{l}\text { dependent incremental } \\
\text { constructive replication }\end{array}$ \\
\hline 448 & $\mathrm{JOM}$ & full text & $\begin{array}{l}\text { Greenbaum, } \\
\text { Mawritz, \& Piccolo } \\
(2015)\end{array}$ & $\begin{array}{l}\text { study } 2 \text { of study } \\
1\end{array}$ & $\begin{array}{l}\text { replication or } \\
\text { replicate }\end{array}$ & dependent & $\begin{array}{l}\text { dependent confounded } \\
\text { replication }\end{array}$ \\
\hline 449 & JOM & full text & $\begin{array}{l}\text { Harrison, Banks, } \\
\text { Pollack, O’Boyle, } \\
\text { \& Short (2017) }\end{array}$ & $\begin{array}{l}\text { previous } \\
\text { research }\end{array}$ & $\begin{array}{l}\text { replication or } \\
\text { replicate }\end{array}$ & independent & $\begin{array}{l}\text { independent literal } \\
\text { reproducibility }\end{array}$ \\
\hline
\end{tabular}




\begin{tabular}{|c|c|c|c|c|c|c|c|}
\hline \# & Journal & Search & Study & $\begin{array}{l}\text { Which } \\
\text { repetition }\end{array}$ & Terminology & Independence & $\begin{array}{l}\text { Type of replication or } \\
\text { reproducibility study }\end{array}$ \\
\hline 450 & JOM & full text & $\begin{array}{l}\text { Hartog, Boon, } \\
\text { Verburg, \& Croon } \\
(2013)\end{array}$ & $\begin{array}{l}\text { previous } \\
\text { research }\end{array}$ & $\begin{array}{l}\text { replication or } \\
\text { replicate }\end{array}$ & independent & $\begin{array}{l}\text { independent quasi-random } \\
\text { replication }\end{array}$ \\
\hline 451 & JOM & full text & $\begin{array}{l}\text { Heavey \& Simsek } \\
(2017)\end{array}$ & study 1 & $\begin{array}{l}\text { constructive } \\
\text { replication }\end{array}$ & dependent & $\begin{array}{l}\text { dependent quasi-random } \\
\text { reproducibility }\end{array}$ \\
\hline 452 & JOM & $\begin{array}{l}\text { abstract } \\
\& \text { title }\end{array}$ & $\begin{array}{l}\text { Hochwarter, Ferris, } \\
\text { Laird, Treadway, \& } \\
\text { Gallagher (2010) }\end{array}$ & $\begin{array}{l}\text { sample } 2 \text { of } \\
\text { sample } 1\end{array}$ & $\begin{array}{l}\text { replication or } \\
\text { replicate }\end{array}$ & dependent & $\begin{array}{l}\text { dependent quasi-random } \\
\text { replication }\end{array}$ \\
\hline 453 & JOM & $\begin{array}{l}\text { abstract } \\
\& \text { title }\end{array}$ & $\begin{array}{l}\text { Hochwarter, Ferris, } \\
\text { Laird, Treadway, \& } \\
\text { Gallagher (2010) }\end{array}$ & $\begin{array}{l}\text { sample } 4 \text { of } \\
\text { sample } 3\end{array}$ & $\begin{array}{l}\text { replication or } \\
\text { replicate }\end{array}$ & dependent & $\begin{array}{l}\text { dependent quasi-random } \\
\text { replication }\end{array}$ \\
\hline 454 & $\mathrm{JOM}$ & $\begin{array}{l}\text { abstract } \\
\& \text { title }\end{array}$ & $\begin{array}{l}\text { Hochwarter, Ferris, } \\
\text { Laird, Treadway, \& } \\
\text { Gallagher (2010) }\end{array}$ & sample 5 & nothing & dependent & generalizability \\
\hline 455 & JOM & $\begin{array}{l}\text { abstract } \\
\& \text { title }\end{array}$ & $\begin{array}{l}\text { Hochwarter, Laird, } \\
\text { \& Brouer (2008) }\end{array}$ & $\begin{array}{l}\text { sample } 2 \text { of } \\
\text { sample } 1\end{array}$ & $\begin{array}{l}\text { replication or } \\
\text { replicate }\end{array}$ & dependent & $\begin{array}{l}\text { dependent quasi-random } \\
\text { replication }\end{array}$ \\
\hline 456 & JOM & $\begin{array}{l}\text { abstract } \\
\& \text { title }\end{array}$ & $\begin{array}{l}\text { Hochwarter, Laird, } \\
\text { \& Brouer (2008) }\end{array}$ & $\begin{array}{l}\text { sample } 3 \text { of } \\
\text { samples } 1 \text { and } 2\end{array}$ & $\begin{array}{l}\text { replication or } \\
\text { replicate }\end{array}$ & dependent & $\begin{array}{l}\text { dependent quasi-random } \\
\text { replication }\end{array}$ \\
\hline 457 & JOM & $\begin{array}{l}\text { abstract } \\
\& \text { title }\end{array}$ & $\begin{array}{l}\text { Hochwarter, Laird, } \\
\text { \& Brouer (2008) }\end{array}$ & $\begin{array}{l}\text { sample } 4 \text { of } \\
\text { samples } 1-3\end{array}$ & $\begin{array}{l}\text { replication or } \\
\text { replicate }\end{array}$ & dependent & $\begin{array}{l}\text { dependent quasi-random } \\
\text { replication }\end{array}$ \\
\hline
\end{tabular}




\begin{tabular}{|c|c|c|c|c|c|c|c|}
\hline \# & Journal & Search & Study & $\begin{array}{l}\text { Which } \\
\text { repetition }\end{array}$ & Terminology & Independence & $\begin{array}{l}\text { Type of replication or } \\
\text { reproducibility study }\end{array}$ \\
\hline 458 & JOM & $\begin{array}{l}\text { abstract } \\
\& \text { title }\end{array}$ & $\begin{array}{l}\text { Hochwarter, Laird, } \\
\text { \& Brouer (2008) }\end{array}$ & $\begin{array}{l}\text { sample } 5 \text { of } \\
\text { samples } 1-4\end{array}$ & $\begin{array}{l}\text { replication or } \\
\text { replicate }\end{array}$ & dependent & $\begin{array}{l}\text { dependent quasi-random } \\
\text { replication }\end{array}$ \\
\hline 459 & $\mathrm{JOM}$ & full text & $\begin{array}{l}\text { Holtz \& Harold } \\
(2013)\end{array}$ & $\begin{array}{l}\text { study } 2 \text { of study } \\
1\end{array}$ & $\begin{array}{l}\text { replication or } \\
\text { replicate }\end{array}$ & dependent & $\begin{array}{l}\text { dependent incremental } \\
\text { constructive replication }\end{array}$ \\
\hline 460 & JOM & full text & $\begin{array}{l}\text { Houwelingen, } \\
\text { Dijke, \& Cremer } \\
(2017)\end{array}$ & $\begin{array}{l}\text { study } 3 \text { of study } \\
1\end{array}$ & $\begin{array}{l}\text { replication or } \\
\text { replicate }\end{array}$ & dependent & $\begin{array}{l}\text { dependent quasi-random } \\
\text { replication }\end{array}$ \\
\hline 461 & JOM & full text & $\begin{array}{l}\text { Houwelingen, } \\
\text { Dijke, \& Cremer } \\
(2017)\end{array}$ & $\begin{array}{l}\text { study } 4 \text { of study } \\
1 \text { and } 3\end{array}$ & $\begin{array}{l}\text { replication or } \\
\text { replicate }\end{array}$ & dependent & $\begin{array}{l}\text { dependent quasi-random } \\
\text { replication }\end{array}$ \\
\hline 462 & JOM & full text & $\begin{array}{l}\text { Houwelingen, } \\
\text { Dijke, \& Cremer } \\
(2017)\end{array}$ & $\begin{array}{l}\text { study } 2 \text { of study } \\
1\end{array}$ & nothing & dependent & $\begin{array}{l}\text { dependent confounded } \\
\text { replication }\end{array}$ \\
\hline 463 & JOM & full text & $\begin{array}{l}\text { Hüffmeier, Freund, } \\
\text { Zerres, Backhaus, } \\
\text { \& Hertel (2014) }\end{array}$ & study 1 & $\begin{array}{l}\text { replication or } \\
\text { replicate }\end{array}$ & independent & $\begin{array}{l}\text { independent quasi-random } \\
\text { replication }\end{array}$ \\
\hline 464 & $\mathrm{JOM}$ & full text & $\begin{array}{l}\text { Kam, Morin, } \\
\text { Meyer, \& } \\
\text { Topolnytsky (2016) }\end{array}$ & $\begin{array}{l}\text { previous } \\
\text { research }\end{array}$ & $\begin{array}{l}\text { replication or } \\
\text { replicate }\end{array}$ & independent & $\begin{array}{l}\text { independent quasi-random } \\
\text { replication }\end{array}$ \\
\hline 465 & $\mathrm{JOM}$ & full text & $\begin{array}{l}\text { King, Botsford, } \\
\text { Hebl, Kazama, }\end{array}$ & $\begin{array}{l}\text { study } 5 \text { of } \\
\text { studies } 3 \text { and } 4\end{array}$ & $\begin{array}{l}\text { replication or } \\
\text { replicate }\end{array}$ & dependent & $\begin{array}{l}\text { dependent quasi-random } \\
\text { replication }\end{array}$ \\
\hline
\end{tabular}




\begin{tabular}{|c|c|c|c|c|c|c|c|}
\hline \# & Journal & Search & Study & $\begin{array}{l}\text { Which } \\
\text { repetition }\end{array}$ & Terminology & Independence & $\begin{array}{l}\text { Type of replication or } \\
\text { reproducibility study }\end{array}$ \\
\hline & & & $\begin{array}{l}\text { Dawson, \& Perkins } \\
\text { (2012) }\end{array}$ & & & & \\
\hline 466 & JOM & full text & $\begin{array}{l}\text { King, Botsford, } \\
\text { Hebl, Kazama, } \\
\text { Dawson, \& Perkins } \\
\text { (2012) }\end{array}$ & $\begin{array}{l}\text { study } 2 \text { of study } \\
1\end{array}$ & $\begin{array}{l}\text { generalizabilit } \\
\text { y }\end{array}$ & dependent & $\begin{array}{l}\text { dependent quasi-random } \\
\text { replication }\end{array}$ \\
\hline 467 & $\mathrm{JOM}$ & full text & $\begin{array}{l}\text { King, Mohr, } \\
\text { Peddie, Jones, \& } \\
\text { Kendra (2017) }\end{array}$ & $\begin{array}{l}\text { previous } \\
\text { research }\end{array}$ & $\begin{array}{l}\text { replication or } \\
\text { replicate }\end{array}$ & independent & $\begin{array}{l}\text { independent quasi-random } \\
\text { replication }\end{array}$ \\
\hline 468 & JOM & full text & $\begin{array}{l}\text { Kluemper, } \\
\text { DeGroot, \& Choi } \\
\text { (2013) }\end{array}$ & $\begin{array}{l}\text { previous } \\
\text { research }\end{array}$ & $\begin{array}{l}\text { replication or } \\
\text { replicate }\end{array}$ & independent & $\begin{array}{l}\text { independent quasi-random } \\
\text { replication }\end{array}$ \\
\hline 469 & JOM & full text & $\begin{array}{l}\text { Kluemper, } \\
\text { DeGroot, \& Choi } \\
\text { (2013) }\end{array}$ & $\begin{array}{l}\text { study } 2 \text { of study } \\
1\end{array}$ & $\begin{array}{l}\text { replication or } \\
\text { replicate }\end{array}$ & dependent & $\begin{array}{l}\text { dependent quasi-random } \\
\text { replication }\end{array}$ \\
\hline 470 & JOM & full text & $\begin{array}{l}\text { Kostopoulos, } \\
\text { Spanos, \& } \\
\text { Prastacos (2013) }\end{array}$ & $\begin{array}{l}\text { study } 3 \text { of study } \\
2\end{array}$ & $\begin{array}{l}\text { replication or } \\
\text { replicate }\end{array}$ & dependent & $\begin{array}{l}\text { dependent quasi-random } \\
\text { replication }\end{array}$ \\
\hline 471 & JOM & full text & $\begin{array}{l}\text { Kukenberger, } \\
\text { Mathieu, \& Ruddy } \\
\text { (2015) }\end{array}$ & $\begin{array}{l}\text { study } 2 \text { of study } \\
1\end{array}$ & $\begin{array}{l}\text { constructive } \\
\text { replication }\end{array}$ & dependent & $\begin{array}{l}\text { dependent substantial } \\
\text { constructive replication }\end{array}$ \\
\hline
\end{tabular}




\begin{tabular}{|c|c|c|c|c|c|c|c|}
\hline \# & Journal & Search & Study & $\begin{array}{l}\text { Which } \\
\text { repetition }\end{array}$ & Terminology & Independence & $\begin{array}{l}\text { Type of replication or } \\
\text { reproducibility study }\end{array}$ \\
\hline 472 & $\mathrm{JOM}$ & full text & $\begin{array}{l}\text { Kunze, Jong, \& } \\
\text { Bruch (2016) }\end{array}$ & $\begin{array}{l}\text { footnotes } 3 \text { and } \\
9\end{array}$ & $\begin{array}{l}\text { replication or } \\
\text { replicate }\end{array}$ & dependent & $\begin{array}{l}\text { dependent quasi-random } \\
\text { reproducibility }\end{array}$ \\
\hline 473 & $\mathrm{JOM}$ & full text & $\begin{array}{l}\text { Lanzolla \& Suarez } \\
(2012)\end{array}$ & study 1 & $\begin{array}{l}\text { replication or } \\
\text { replicate }\end{array}$ & dependent & $\begin{array}{l}\text { dependent quasi-random } \\
\text { reproducibility }\end{array}$ \\
\hline 474 & JOM & full text & $\begin{array}{l}\text { Lapierre \& Allen } \\
(2012)\end{array}$ & $\begin{array}{l}\text { previous } \\
\text { research }\end{array}$ & $\begin{array}{l}\text { replication or } \\
\text { replicate }\end{array}$ & independent & $\begin{array}{l}\text { independent quasi-random } \\
\text { replication }\end{array}$ \\
\hline 475 & JOM & full text & $\begin{array}{l}\text { Lee \& Antonakis } \\
(2014)\end{array}$ & study 1 & $\begin{array}{l}\text { replication or } \\
\text { replicate }\end{array}$ & dependent & generalizability \\
\hline 476 & $\mathrm{JOM}$ & $\begin{array}{l}\text { abstract } \\
\& \text { title }\end{array}$ & $\begin{array}{l}\text { Livingston, } \\
\text { Schilpzand, \& Erez } \\
(2017)\end{array}$ & $\begin{array}{l}\text { sample } 2 \text { of } \\
\text { sample } 1 \text { in } \\
\text { study } 1\end{array}$ & nothing & dependent & $\begin{array}{l}\text { dependent quasi-random } \\
\text { replication }\end{array}$ \\
\hline 477 & $\mathrm{JOM}$ & $\begin{array}{l}\text { abstract } \\
\& \text { title }\end{array}$ & $\begin{array}{l}\text { Livingston, } \\
\text { Schilpzand, \& Erez } \\
\text { (2017) }\end{array}$ & study 2 of study & $\begin{array}{l}\text { replication or } \\
\text { replicate }\end{array}$ & dependent & $\begin{array}{l}\text { dependent quasi-random } \\
\text { replication }\end{array}$ \\
\hline 478 & JOM & $\begin{array}{l}\text { abstract } \\
\& \text { title }\end{array}$ & $\begin{array}{l}\text { Livingston, } \\
\text { Schilpzand, \& Erez } \\
(2017)\end{array}$ & $\begin{array}{l}\text { study } 3 \text { of } \\
\text { studies } 1 \text { and } 2\end{array}$ & $\begin{array}{l}\text { replication or } \\
\text { replicate }\end{array}$ & dependent & generalizability \\
\hline 479 & JOM & full text & Madsen (2013) & study 1 & $\begin{array}{l}\text { replication or } \\
\text { replicate }\end{array}$ & dependent & $\begin{array}{l}\text { dependent quasi-random } \\
\text { reproducibility }\end{array}$ \\
\hline
\end{tabular}




\begin{tabular}{|c|c|c|c|c|c|c|c|}
\hline \# & Journal & Search & Study & $\begin{array}{l}\text { Which } \\
\text { repetition }\end{array}$ & Terminology & Independence & $\begin{array}{l}\text { Type of replication or } \\
\text { reproducibility study }\end{array}$ \\
\hline 480 & $\mathrm{JOM}$ & full text & $\begin{array}{l}\text { Marcus, Taylor, } \\
\text { Hastings, Sturm, \& } \\
\text { Weigelt (2016) }\end{array}$ & $\begin{array}{l}\text { study } 2 \text { of study } \\
1\end{array}$ & $\begin{array}{l}\text { replication or } \\
\text { replicate }\end{array}$ & dependent & $\begin{array}{l}\text { dependent quasi-random } \\
\text { replication }\end{array}$ \\
\hline 481 & $\mathrm{JOM}$ & $\begin{array}{l}\text { abstract } \\
\& \text { title }\end{array}$ & $\begin{array}{l}\text { McClelland, Liang, } \\
\text { \& Barker (2010) }\end{array}$ & $\begin{array}{l}\text { previous } \\
\text { research }\end{array}$ & $\begin{array}{l}\text { replication or } \\
\text { replicate }\end{array}$ & independent & $\begin{array}{l}\text { independent quasi-random } \\
\text { replication }\end{array}$ \\
\hline 482 & $\mathrm{JOM}$ & full text & $\begin{array}{l}\text { Perry, Lorinkova, } \\
\text { Hunter, Hubbard, \& } \\
\text { McMahon (2016) }\end{array}$ & $\begin{array}{l}\text { study } 2 \text { of study } \\
1\end{array}$ & $\begin{array}{l}\text { replication or } \\
\text { replicate }\end{array}$ & dependent & dependent literal replication \\
\hline 483 & JOM & full text & $\begin{array}{l}\text { Peters, Daniels, } \\
\text { Hodgkinson, \& } \\
\text { Haslam (2014) }\end{array}$ & study 1 & $\begin{array}{l}\text { replication or } \\
\text { replicate }\end{array}$ & dependent & $\begin{array}{l}\text { dependent quasi-random } \\
\text { reproducibility }\end{array}$ \\
\hline 484 & $\mathrm{JOM}$ & full text & $\begin{array}{l}\text { Quinn \& } \\
\text { Bunderson (2016) }\end{array}$ & $\begin{array}{l}\text { previous } \\
\text { research }\end{array}$ & $\begin{array}{l}\text { replication or } \\
\text { replicate }\end{array}$ & independent & generalizability \\
\hline 485 & JOM & $\begin{array}{l}\text { abstract } \\
\& \text { title }\end{array}$ & $\begin{array}{l}\text { Restubog, } \\
\text { Zagenczyk, Bordia, } \\
\text { Bordia, \& Chapman } \\
(2015)\end{array}$ & $\begin{array}{l}\text { Sample } 2 \text { of } \\
\text { sample } 1\end{array}$ & $\begin{array}{l}\text { constructive } \\
\text { replication }\end{array}$ & dependent & $\begin{array}{l}\text { dependent regressive } \\
\text { replication }\end{array}$ \\
\hline 486 & JOM & $\begin{array}{l}\text { abstract } \\
\& \text { title }\end{array}$ & $\begin{array}{l}\text { Restubog, } \\
\text { Zagenczyk, Bordia, } \\
\text { Bordia, \& Chapman } \\
(2015)\end{array}$ & $\begin{array}{l}\text { previous } \\
\text { research }\end{array}$ & $\begin{array}{l}\text { replication or } \\
\text { replicate }\end{array}$ & independent & $\begin{array}{l}\text { semi-independent quasi- } \\
\text { random replication }\end{array}$ \\
\hline
\end{tabular}




\begin{tabular}{|c|c|c|c|c|c|c|c|}
\hline \# & Journal & Search & Study & $\begin{array}{l}\text { Which } \\
\text { repetition }\end{array}$ & Terminology & Independence & $\begin{array}{l}\text { Type of replication or } \\
\text { reproducibility study }\end{array}$ \\
\hline 487 & $\mathrm{JOM}$ & $\begin{array}{l}\text { abstract } \\
\& \text { title }\end{array}$ & $\begin{array}{l}\text { Rosen, Slater, } \\
\text { Chang, \& Johnson } \\
\text { (2013) }\end{array}$ & $\begin{array}{l}\text { study } 4 \text { of } \\
\text { studies } 2 \text { and } 3\end{array}$ & $\begin{array}{l}\text { replication or } \\
\text { replicate }\end{array}$ & dependent & $\begin{array}{l}\text { dependent incremental } \\
\text { constructive replication }\end{array}$ \\
\hline 488 & JOM & $\begin{array}{l}\text { abstract } \\
\& \text { title }\end{array}$ & $\begin{array}{l}\text { Rosen, Slater, } \\
\text { Chang, \& Johnson } \\
(2013)\end{array}$ & $\begin{array}{l}\text { study } 3 \text { of study } \\
2\end{array}$ & $\begin{array}{l}\text { replication or } \\
\text { replicate }\end{array}$ & dependent & $\begin{array}{l}\text { dependent quasi-random } \\
\text { replication }\end{array}$ \\
\hline 489 & JOM & full text & $\begin{array}{l}\text { Rosenbusch, } \\
\text { Rauch, \& Bausch } \\
(2013)\end{array}$ & study 1 & $\begin{array}{l}\text { replication or } \\
\text { replicate }\end{array}$ & dependent & $\begin{array}{l}\text { dependent quasi-random } \\
\text { reproducibility }\end{array}$ \\
\hline 490 & $\mathrm{JOM}$ & full text & $\begin{array}{l}\text { Shen \& Benson } \\
(2016)\end{array}$ & $\begin{array}{l}\text { previous } \\
\text { research }\end{array}$ & $\begin{array}{l}\text { replication or } \\
\text { replicate }\end{array}$ & independent & $\begin{array}{l}\text { independent quasi-random } \\
\text { replication }\end{array}$ \\
\hline 491 & $\mathrm{JOM}$ & full text & $\begin{array}{l}\text { Steel, Kammeyer- } \\
\text { Mueller, \& } \\
\text { Paterson (2015) }\end{array}$ & $\begin{array}{l}\text { previous } \\
\text { research }\end{array}$ & $\begin{array}{l}\text { replication or } \\
\text { replicate }\end{array}$ & dependent & $\begin{array}{l}\text { dependent substantial } \\
\text { constructive replication }\end{array}$ \\
\hline 492 & $\mathrm{JOM}$ & $\begin{array}{l}\text { abstract } \\
\text { \& title }\end{array}$ & $\begin{array}{l}\text { Sue-Chan, Wood, } \\
\text { \& Latham (2012) }\end{array}$ & $\begin{array}{l}\text { study } 2 \text { of study } \\
1\end{array}$ & $\begin{array}{l}\text { replication or } \\
\text { replicate }\end{array}$ & dependent & $\begin{array}{l}\text { dependent quasi-random } \\
\text { replication }\end{array}$ \\
\hline 493 & $\mathrm{JOM}$ & $\begin{array}{l}\text { abstract } \\
\text { \& title }\end{array}$ & $\begin{array}{l}\text { Treadway, Breland, } \\
\text { Williams, Cho, } \\
\text { Yang, \& Ferris } \\
(2013)\end{array}$ & $\begin{array}{l}\text { study } 2 \text { of study } \\
1\end{array}$ & $\begin{array}{l}\text { constructive } \\
\text { replication }\end{array}$ & dependent & $\begin{array}{l}\text { dependent quasi-random } \\
\text { replication }\end{array}$ \\
\hline
\end{tabular}




\begin{tabular}{|c|c|c|c|c|c|c|c|}
\hline \# & Journal & Search & Study & $\begin{array}{l}\text { Which } \\
\text { repetition }\end{array}$ & Terminology & Independence & $\begin{array}{l}\text { Type of replication or } \\
\text { reproducibility study }\end{array}$ \\
\hline 494 & JOM & full text & Vagnani (2015) & footnote 8 & $\begin{array}{l}\text { replication or } \\
\text { replicate }\end{array}$ & dependent & $\begin{array}{l}\text { dependent quasi-random } \\
\text { reproducibility }\end{array}$ \\
\hline 495 & JOM & full text & $\begin{array}{l}\text { Vogel \& Mitchell } \\
(2017)\end{array}$ & $\begin{array}{l}\text { study } 2 \text { of study } \\
1\end{array}$ & $\begin{array}{l}\text { constructive } \\
\text { replication }\end{array}$ & dependent & $\begin{array}{l}\text { dependent substantial } \\
\text { constructive replication }\end{array}$ \\
\hline 496 & JOM & full text & $\begin{array}{l}\text { Vogel \& Mitchell } \\
(2017)\end{array}$ & $\begin{array}{l}\text { study } 3 \text { of } \\
\text { studies } 1 \text { and } 2\end{array}$ & $\begin{array}{l}\text { replication or } \\
\text { replicate }\end{array}$ & dependent & $\begin{array}{l}\text { dependent confounded } \\
\text { replication }\end{array}$ \\
\hline 497 & JOM & full text & $\begin{array}{l}\text { Wallace, Butts, } \\
\text { Johnson, Stevens, } \\
\text { \& Smith (2016) }\end{array}$ & $\begin{array}{l}\text { previous } \\
\text { research }\end{array}$ & $\begin{array}{l}\text { replication or } \\
\text { replicate }\end{array}$ & independent & $\begin{array}{l}\text { independent quasi-random } \\
\text { replication }\end{array}$ \\
\hline 498 & $\mathrm{JOM}$ & full text & $\begin{array}{l}\text { Wallace, Edwards, } \\
\text { Paul, Burke, } \\
\text { Christian, \& Eissa } \\
\text { (2016) }\end{array}$ & $\begin{array}{l}\text { previous } \\
\text { research }\end{array}$ & $\begin{array}{l}\text { replication or } \\
\text { replicate }\end{array}$ & independent & $\begin{array}{l}\text { independent quasi-random } \\
\text { replication }\end{array}$ \\
\hline 499 & JOM & full text & $\begin{array}{l}\text { Way, Tracey, Fay, } \\
\text { Wright, Snell, } \\
\text { Chang, \& Gong } \\
\text { (2015) }\end{array}$ & $\begin{array}{l}\text { sample } 7 \text { of } \\
\text { previous } \\
\text { samples }\end{array}$ & $\begin{array}{l}\text { replication or } \\
\text { replicate }\end{array}$ & dependent & $\begin{array}{l}\text { dependent quasi-random } \\
\text { replication }\end{array}$ \\
\hline 500 & JOM & $\begin{array}{l}\text { abstract } \\
\& \text { title }\end{array}$ & $\begin{array}{l}\text { Wihler, Blickle, } \\
\text { Parker Ellen, } \\
\text { Hochwarter, \& } \\
\text { Ferris (2017) }\end{array}$ & $\begin{array}{l}\text { study } 3 \text { of } \\
\text { studies } 1 \text { and } 2\end{array}$ & $\begin{array}{l}\text { constructive } \\
\text { replication }\end{array}$ & dependent & $\begin{array}{l}\text { dependent quasi-random } \\
\text { replication }\end{array}$ \\
\hline
\end{tabular}




\begin{tabular}{|c|c|c|c|c|c|c|c|}
\hline \# & Journal & Search & Study & $\begin{array}{l}\text { Which } \\
\text { repetition }\end{array}$ & Terminology & Independence & $\begin{array}{l}\text { Type of replication or } \\
\text { reproducibility study }\end{array}$ \\
\hline 501 & $\mathrm{JOM}$ & full text & $\begin{array}{l}\text { Wu \& Parker } \\
(2017)\end{array}$ & $\begin{array}{l}\text { study } 2 \text { of study } \\
1\end{array}$ & $\begin{array}{l}\text { replication or } \\
\text { replicate }\end{array}$ & dependent & $\begin{array}{l}\text { dependent quasi-random } \\
\text { replication }\end{array}$ \\
\hline 502 & $\mathrm{JOM}$ & full text & $\begin{array}{l}\text { Wu, Parker, \& Jong } \\
(2014)\end{array}$ & $\begin{array}{l}\text { study } \\
\text { mentioned in } \\
\text { discussion } \\
\text { section }\end{array}$ & $\begin{array}{l}\text { replication or } \\
\text { replicate }\end{array}$ & dependent & $\begin{array}{l}\text { dependent quasi-random } \\
\text { replication }\end{array}$ \\
\hline 503 & $\mathrm{JOM}$ & full text & $\begin{array}{l}\text { Young, Morris, \& } \\
\text { Scherwin (2013) }\end{array}$ & $\begin{array}{l}\text { study } 3 \text { of study } \\
2\end{array}$ & $\begin{array}{l}\text { replication or } \\
\text { replicate }\end{array}$ & dependent & $\begin{array}{l}\text { dependent quasi-random } \\
\text { replication }\end{array}$ \\
\hline 504 & $\mathrm{JOM}$ & full text & $\begin{array}{l}\text { Zagenczyk, } \\
\text { Restubog, Kiewitz, } \\
\text { Kiazad, \& Tang } \\
(2014)\end{array}$ & $\begin{array}{l}\text { study } 3 \text { of } \\
\text { studies } 1 \text { and } 2\end{array}$ & $\begin{array}{l}\text { constructive } \\
\text { replication }\end{array}$ & dependent & $\begin{array}{l}\text { dependent quasi-random } \\
\text { replication }\end{array}$ \\
\hline 505 & JOM & full text & $\begin{array}{l}\text { Zagenczyk, } \\
\text { Restubog, Kiewitz, } \\
\text { Kiazad, \& Tang } \\
(2014)\end{array}$ & $\begin{array}{l}\text { study } 4 \text { of } \\
\text { studies } 1-3\end{array}$ & $\begin{array}{l}\text { constructive } \\
\text { replication }\end{array}$ & dependent & $\begin{array}{l}\text { dependent quasi-random } \\
\text { replication }\end{array}$ \\
\hline 506 & $\mathrm{JOM}$ & full text & $\begin{array}{l}\text { Zagenczyk, } \\
\text { Restubog, Kiewitz, } \\
\text { Kiazad, \& Tang } \\
(2014)\end{array}$ & $\begin{array}{l}\text { study } 2 \text { of study } \\
1\end{array}$ & $\begin{array}{l}\text { constructive } \\
\text { replication }\end{array}$ & dependent & generalizability \\
\hline 507 & JOM & full text & $\begin{array}{l}\text { Zaheer, Castañer, \& } \\
\text { Souder (2013) }\end{array}$ & $\begin{array}{l}\text { previous } \\
\text { research }\end{array}$ & $\begin{array}{l}\text { replication or } \\
\text { replicate }\end{array}$ & independent & $\begin{array}{l}\text { independent quasi-random } \\
\text { replication }\end{array}$ \\
\hline
\end{tabular}




\begin{tabular}{|l|l|l|l|l|l|l|l|}
\hline$\#$ & Journal & Search & Study & $\begin{array}{l}\text { Which } \\
\text { repetition }\end{array}$ & Terminology & Independence & $\begin{array}{l}\text { Type of replication or } \\
\text { reproducibility study }\end{array}$ \\
\hline 508 & JOM & full text & $\begin{array}{l}\text { Zhang, Li, Ullrich, } \\
\text { \& Dick (2015) }\end{array}$ & $\begin{array}{l}\text { previous } \\
\text { research }\end{array}$ & $\begin{array}{l}\text { replication or } \\
\text { replicate }\end{array}$ & independent & $\begin{array}{l}\text { independent quasi-random } \\
\text { replication }\end{array}$ \\
\hline
\end{tabular}




\title{
Supplementary Materials B: References for coded articles
}

\begin{abstract}
AMJ
Ballesteros, L., Useem, M. \& Wry, T. 2017. Masters of disasters? An empirical analysis of how societies benefit from corporate disaster aid. Academy of Management Journal, 60: 16821708.
\end{abstract}

Barrick, M. R., Bradley, B. H., Kristof-Brown, A. L. \& Colbert, A. E. 2007. The moderating role of top management team interdependence: Implications for real teams and working groups. Academy of Management Journal, 50: 544-557.

Bendersky, C. \& Parikh Shah, N. 2013. The downfall of extraverts and rise of neurotics: The dynamic process of status allocation in task groups. Academy of Management Journal, 56: $387-406$.

Boivie, S., Graffin, S. D. \& Pollock, T. G. 2012. Time for me to fly: Predicting director exit at large firms. Academy of Management Journal, 55: 1334-1359.

Briscoe, F., Chin, M. K. \& Hambrick, D. C. 2014. CEO ideology as an element of the corporate opportunity structure for social activists. Academy of Management Journal, 57: 17861809.

Briscoe, F. \& Joshi, A. 2017. Bringing the boss's politics in: Supervisor political ideology and the gender gap in earnings. Academy of Management Journal, 60: 1415-1441.

Carson Marr, J. \& Cable, D. M. 2014. Do interviewers sell themselves short? The effects of selling orientation on interviewers' judgments. Academy of Management Journal, 57: 624-651. 
Carson Marr, J. \& Thau, S. 2014. Falling from great (and not-so-great) heights: How initial status position influences performance after status loss. Academy of Management Journal, 57: 223-248.

Carton, A. M., Murphy, C. \& Clark, J. R. 2014. A (blurry) vision of the future: How leader rhetoric about ultimate goals influences performance. Academy of Management Journal, 57: $1544-1570$.

Černe, M., Nerstad, C. G. L., Dysvik, A. \& Škerlavaj, M. 2014. What goes around comes around: Knowledge hiding, perceived motivational climate, and creativity. Academy of Management Journal, 57: 172-192.

Chen, Y., Ferris, D. L., Kwan, H. K., Yan, M., Zhou, M. \& Hong, Y. 2013. Self-love's lost labor: A self-enhancement model of workplace incivility. Academy of Management Journal, 56: 1199-1219.

Courtright, S. H., Gardner, R. G., Smith, T. A., McCormick, B. W. \& Colbert, A. E. 2016. My family made me do it: A cross-domain, self-regulatory perspective on antecedents to abusive supervision. Academy of Management Journal, 59: 1630-1652.

Crilly, D., Hansen, M. \& Zollo, M. 2016. The grammar of decoupling: A cognitive-linguistic perspective on firms' sustainability claims and stakeholders' interpretation. Academy of Management Journal, 59: 705-729.

De Vries, T. A., Walter, F., Van Der Vegt, G. S. \& Essens, P. J. M. D. 2014. Antecedents of individuals' interteam coordination: Broad functional experiences as a mixed blessing. Academy of Management Journal, 57: 1334-1359. 
Desai, V. 2015. Learning through the distribution of failures within an organization: Evidence from heart bypass surgery performance. Academy of Management Journal, 58: 10321050.

Desai, V. M. 2016. The behavioral theory of the (governed) firm: Corporate board influences on organizations' responses to performance shortfalls. Academy of Management Journal, 59: 860-879.

Dineen, B. R., Duffy, M. K., Henle, C. A. \& Kiyoung, L. E. E. 2017. Green by comparison: Deviant and normative transmutations of job search envy in a temporal context. Academy of Management Journal, 60: 295-320.

Dineen, B. R., Noe, R. A., Shaw, J. D., Duffy, M. K. \& Wiethoff, C. 2007. Level and dispersion of satisfaction in teams: Using foci and social context to explain the satisfactionabsenteeism relationship. Academy of Management Journal, 50: 623-643.

Duffy, M. K., Scott, K. L., Shaw, J. D., Tepper, B. J. \& Aquino, K. 2012. A social context model of envy and social undermining. Academy of Management Journal, 55: 643-666.

Erez, A., Schilpzand, P., Leavitt, K., Woolum, A. H. \& Judge, T. A. 2015. Inherently relational: Interactions between peers' and individuals' personalities impact reward giving and appraisal of individual performance. Academy of Management Journal, 58: 1761-1784.

Faison Hewlin, P., Dumas, T. L. \& Flowers Burnett, M. 2017. To thine own self be true? Facades of conformity, values incongruence, and the moderating impact of leader integrity. Academy of Management Journal, 60: 178-199.

Farh, C. I. C., Lanaj, K. \& Ilies, R. 2017. Resource-based contingencies of when team-member exchange helps member performance in teams. Academy of Management Journal, 60: 1117-1137. 
Farooq, O., Rupp, D. E. \& Farooq, M. 2017. The multiple pathways through which internal and external corporate social responsibility influence organizational identification and multifoci outcomes: The moderating role of cultural and social orientations. Academy of Management Journal, 60: 954-985.

Ferris, D. L., Lian, H., Brown, D. J. \& Morrison, R. 2015. Ostracism, self-esteem, and job performance: When do we self-verify and when do we self-enhance? Academy of Management Journal, 58: 279-297.

Ferris, D. L., Yan, M., Lim, V. K. G., Chen, Y. \& Fatimah, S. 2016. An approach-avoidance framework of workplace aggression. Academy of Management Journal, 59: 1777-1800.

Firth, B. M., Chen, G., Kirkman, B. L. \& Kim, K. 2014. Newcomers abroad: Expatriate adaptation during early phases of international assignments. Academy of Management Journal, 57: 280-300.

Flammer, C. 2013. Corporate social responsibility and shareholder reaction: The environmental awareness of investors. Academy of Management Journal, 56: 758-781.

Gabriel, A. S. \& Diefendorff, J. M. 2015. Emotional labor dynamics: A momentary approach. Academy of Management Journal, 58: 1804-1825.

Galunic, C., Ertug, G. \& Gargiulo, M. 2012. The positive externalities of social capital: Benefiting from senior brokers. Academy of Management Journal, 55: 1213-1231.

Glaser, L., Stam, W. \& Takeuchi, R. 2016. Managing the risks of proactivity: A multilevel study of initiative and performance in the middle management context. Academy of Management Journal, 59: 1339-1360. 
Grant, A. M. 2012. Leading with meaning: Beneficiary contact, prosocial impact, and the performance effects of transformational leadership. Academy of Management Journal, 55: $458-476$.

Grant, A. M., Gino, F. \& Hofmann, D. A. 2011. Reversing the extraverted leadership advantage: The role of employee proactivity. Academy of Management Journal, 54: 528-550.

Grégoire, D. A. \& Shepherd, D. A. 2012. Technology-market combinations and the identification of entrepreneurial opportunities: An investigation of the opportunityindividual nexus. Academy of Management Journal, 55: 753-785.

Greve, H. R. \& Zhang, C. M. 2017. Institutional logics and power sources: Merger and acquisition decisions. Academy of Management Journal, 60: 671-694.

Grohsjean, T., Kober, P. \& Zucchini, L. 2016. Coming back to edmonton: Competing with former employers and colleagues. Academy of Management Journal, 59: 394-413.

Hafenbrädl, S. \& Waeger, D. 2017. Ideology and the micro-foundations of csr: Why executives believe in the business case for csr and how this affects their csr engagements. Academy of Management Journal, 60: 1582-1606.

Harrison, S. H. \& Wagner, D. T. 2016. Spilling outside the box: The effects of individuals' creative behaviors at work on time spent with their spouses at home. Academy of Management Journal, 59: 841-859.

Heimeriks, K. H., Schijven, M. \& Gates, S. 2012. Manifestations of higher-order routines: The underlying mechanisms of deliberate learning in the context of postacquisition integration. Academy of Management Journal, 55: 703-726.

Jacquart, P. \& Antonakis, J. 2015. When does charisma matter for top-level leaders? Effect of attributional ambiguity. Academy of Management Journal, 58: 1051-1074. 
Jia, H. U. \& Liden, R. C. 2015. Making a difference in the teamwork: Linking team prosocial motivation to team processes and effectiveness. Academy of Management Journal, 58: 1102-1127.

Jones, D. A., Willness, C. R. \& Madey, S. 2014. Why are job seekers attracted by corporate social performance? Experimental and field tests of three signal-based mechanisms. Academy of Management Journal, 57: 383-404.

Kilduff, G. J., Galinsky, A. D., Gallo, E. \& Reade, J. J. 2016. Whatever it takes to win: Rivalry increases unethical behavior. Academy of Management Journal, 59: 1508-1534.

Klingebiel, R. \& Adner, R. O. N. 2015. Real options logic revisited: The performance effects of alternative resource allocation regimes. Academy of Management Journal, 58: 221-241.

Koopman, J., Lanaj, K. \& Scott, B. A. 2016. Integrating the bright and dark sides of ocb: A daily investigation of the benefits and costs of helping others. Academy of Management Journal, 59: 414-435.

Kozhikode, R. K. \& Li, J. 2012. Political pluralism, public policies, and organizational choices: Banking branch expansion in india, 1948-2003. Academy of Management Journal, 55: 339-359.

Kraimer, M. L., Shaffer, M. A., Harrison, D. A. \& Ren, H. 2012. No place like home? An identity strain perspective on repatriate turnover. Academy of Management Journal, 55: $399-420$.

Lam, C. K., Xu, H. \& Chan, S. C. H. 2015. The threshold effect of participative leadership and the role of leader information sharing. Academy of Management Journal, 58: 836-855.

Lanzolla, G. \& Frankort, H. T. W. 2016. The online shadow of offline signals: Which sellers get contacted in online b2b marketplaces? Academy of Management Journal, 59: 207-231. 
Lee, M., Pitesa, M., Pillutla, M. M. \& Thau, S. 2017. Male immorality: An evolutionary account of sex differences in unethical negotiation behavior. Academy of Management Journal, 60: 2014-2044.

Lee, S. Y., Pitesa, M., Thau, S. \& Pillutla, M. M. 2015. Discrimination in selection decisions: Integrating stereotype fit and interdependence theories. Academy of Management Journal, 58: 789-812.

Lepine, M. A., Zhang, Y., Crawford, E. R. \& Rich, B. L. 2016. Turning their pain to gain: Charismatic leader influence on follower stress appraisal and job performance. Academy of Management Journal, 59: 1036-1059.

Leslie, L. M., Flaherty Manchester, C. \& Dahm, P. C. 2017. Why and when does the gender gap reverse? Diversity goals and the pay premium for high potential women. Academy of Management Journal, 60: 402-432.

Lin, B., Law, K. S. \& Zhou, J. 2017. Why is underemployment related to creativity and ocb? A task-crafting explanation of the curvilinear moderated relations. Academy of Management Journal, 60: 156-177.

Little, L. M., Smith Major, V., Hinojosa, A. S. \& Nelson, D. L. 2015. Professional image maintenance: How women navigate pregnancy in the workplace. Academy of Management Journal, 58: 8-37.

Lopez-Kidwell, V., Grosser, T. J., Dineen, B. R. \& Borgatti, S. P. 2013. What matters when: A multistage model and empirical examination of job search effort. Academy of Management Journal, 56: 1655-1678.

Maltarich, M. A., Nyberg, A. J., Reilly, G., Abdulsalam, D. D. \& Martin, M. 2017. Pay-forperformance, sometimes: An interdisciplinary approach to integrating economic 
rationality with psychological emotion to predict individual performance. Academy of Management Journal, 60: 2155-2174.

Matta, F. K., Scott, B. A., Colquitt, J. A., Koopman, J. \& Passantino, L. G. 2017. Is consistently unfair better than sporadically fair? An investigation of justice variability and stress. Academy of Management Journal, 60: 743-770.

Mawritz, M. B., Greenbaum, R. L., Butts, M. M. \& Graham, K. A. 2017. I just can't control myself: A self-regulation perspective on the abuse of deviant employees. Academy of Management Journal, 60: 1482-1503.

Opper, S., Nee, V. \& Holm, H. J. 2017. Risk aversion and guanxi activities: A behavioral analysis of ceos in china. Academy of Management Journal, 60: 1504-1530.

Ou, A. Y., Seo, J., Choi, D. \& Hom, P. W. 2017. When can humble top executives retain middle managers? The moderating role of top management team faultlines. Academy of Management Journal, 60: 1915-1931.

Owens, B. P. \& Hekman, D. R. 2016. How does leader humility influence team performance? Exploring the mechanisms of contagion and collective promotion focus. Academy of Management Journal, 59: 1088-1111.

Parker, S. K., Johnson, A., Collins, C. \& Nguyen, H. 2013. Making the most of structural support: Moderating influence of employees' clarity and negative affect. Academy of Management Journal, 56: 867-892.

Patel, P. C., Messersmith, J. G. \& Lepak, D. P. 2013. Walking the tightrope: An assessment of the relationship between high-performance work systems and organizational ambidexterity. Academy of Management Journal, 56: 1420-1442. 
Pitesa, M. \& Thau, S. 2013. Compliant sinners, obstinate saints: How power and self-focus determine the effectiveness of social influences in ethical decision making. Academy of Management Journal, 56: 635-658.

Raffiee, J. \& Coff, R. 2016. Micro-foundations of firm-specific human capital: When do employees perceive their skills to be firm-specific? Academy of Management Journal, 59: 766-790.

Raffiee, J. \& Feng, J. 2014. Should i quit my day job?: A hybrid path to entrepreneurship. Academy of Management Journal, 57: 936-963.

Ramarajan, L., Rothbard, N. P. \& Wilk, S. L. 2017. Discordant vs. Harmonious selves: The effects of identity conflict and enhancement on sales performance in employee-customer interactions. Academy of Management Journal, 60: 2208-2238.

Reyt, J.-N. \& Wiesenfeld, B. M. 2015. Seeing the forest for the trees: Exploratory learning, mobile technology, and knowledge workers' role integration behaviors. Academy of Management Journal, 58: 739-762.

Rodell, J. B. 2013. Finding meaning through volunteering: Why do employees volunteer and what does it mean for their jobs? Academy of Management Journal, 56: 1274-1294.

Schaubroeck, J. M., Chunyan Peng, A. N. N. \& Hannah, S. T. 2013. Developing trust with peers and leaders: Impacts on organizational identification and performance during entry. Academy of Management Journal, 56: 1148-1168.

Shani, G. U. Y. \& Westphal, J. D. 2016. Persona non grata? Determinants and consequences of social distancing from journalists who engage in negative coverage of firm leadership. Academy of Management Journal, 59: 302-329. 
Shaw, J. D., Dineen, B. R., Fang, R. \& Vellella, R. F. 2009. Employee-organization exchange relationships, hrm practices, and quit rates of good and poor performers. Academy of Management Journal, 52: 1016-1033.

Sirola, N. \& Pitesa, M. 2017. Economic downturns undermine workplace helping by promoting a zero-sum construal of success. Academy of Management Journal, 60: 1339-1359.

Soenen, G., Melkonian, T. \& Ambrose, M. L. 2017. To shift or not to shift? Determinants and consequences of phase shifting on justice judgments. Academy of Management Journal, 60: 798-817.

Stuart, H. C. \& Moore, C. 2017. Shady characters: The implications of illicit organizational roles for resilient team performance. Academy of Management Journal, 60: 1963-1985.

Summers, J. K., Humphrey, S. E. \& Ferris, G. R. 2012. Team member change, flux in coordination, and performance: Effects of strategic core roles, information transfer, and cognitive ability. Academy of Management Journal, 55: 314-338.

Tost, L. P., Gino, F. \& Larrick, R. P. 2013. When power makes others speechless: The negative impact of leader power on team performance. Academy of Management Journal, 56: $1465-1486$.

Trevor, C. O., Reilly, G. \& Gerhart, B. 2012. Reconsidering pay dispersion's effect on the performance of interdependent work: Reconciling sorting and pay inequality. Academy of Management Journal, 55: 585-610.

Vakili, K. \& McGahan, A. M. 2016. Health care's grand challenge: Stimulating basic science on diseases that primarily afflict the poor. Academy of Management Journal, 59: 1917-1939. 
Vincent, L. C. \& Kouchaki, M. 2016. Creative, rare, entitled, and dishonest: How commonality of creativity in one's group decreases an individual's entitlement and dishonesty. Academy of Management Journal, 59: 1451-1473.

Walker, H. J., Bauer, T. N., Cole, M. S., Bernerth, J. B., Feild, H. S. \& Short, J. C. 2013. Is this how i will be treated? Reducing uncertainty through recruitment interactions. Academy of Management Journal, 56: 1325-1347.

Wang, Z., Singh, S. N., Li, Y. J., Mishra, S., Ambrose, M. \& Biernat, M. 2017. Effects of employees' positive affective displays on customer loyalty intentions: An emotions-associal-information perspective. Academy of Management Journal, 60: 109-129.

Wee, E. X. M., Liao, H., Liu, D. \& Liu, J. 2017. Moving from abuse to reconciliation: A powerdependence perspective on when and how a follower can break the spiral of abuse. Academy of Management Journal, 60: 2352-2380.

Wo, D. X. H., Ambrose, M. L. \& Schminke, M. 2015. What drives trickle-down effects? A test of multiple mediation processes. Academy of Management Journal, 58: 1848-1868.

Wombacher, J. \& Felfe, J. 2017. The interplay of team and organizational commitment in motivating employees' interteam conflict handling. Academy of Management Journal, 60: 1554-1581.

Yam, K. C., Klotz, A. C., He, W. \& Reynolds, S. J. 2017. From good soldiers to psychologically entitled: Examining when and why citizenship behavior leads to deviance. Academy of Management Journal, 60: 373-396.

Zapata, C. P., Carton, A. M. \& Liu, J. T. 2016. When justice promotes injustice: Why minority leaders experience bias when they adhere to interpersonal justice rules. Academy of Management Journal, 59: 1150-1173. 
Zhang, Y. \& Qu, H. 2016. The impact of ceo succession with gender change on firm performance and successor early departure: Evidence from china's publicly listed companies in 1997-2010. Academy of Management Journal, 59: 1845-1868.

Zhao, E. Y. \& Wry, T. 2016. Not all inequality is equal: Deconstructing the societal logic of patriarchy to understand microfinance lending to women. Academy of Management Journal, 59: 1994-2020.

\section{JAP}

Adam, H. \& Shirako, A. 2013. Not all anger is created equal: The impact of the expresser's culture on the social effects of anger in negotiations. Journal of Applied Psychology, 98: 785-798.

Ali, A. A., Lyons, B. J. \& Ryan, A. M. 2017. Managing a perilous stigma: Ex-offenders' use of reparative impression management tactics in hiring contexts. Journal of Applied Psychology, 102: 1271-1285.

Ali, A. A., Ryan, A. M., Lyons, B. J., Ehrhart, M. G. \& Wessel, J. L. 2016. The long road to employment: Incivility experienced by job seekers. Journal of Applied Psychology, 101: 333-349.

Allen, D. G., Peltokorpi, V. \& Rubenstein, A. L. 2016. When "embedded" means "stuck": Moderating effects of job embeddedness in adverse work environments. Journal of Applied Psychology, 101: 1670-1686.

Arthur, W., Jr., Glaze, R. M., Jarrett, S. M., White, C. D., Schurig, I. \& Taylor, J. E. 2014. Comparative evaluation of three situational judgment test response formats in terms of 
construct-related validity, subgroup differences, and susceptibility to response distortion: Research report. Journal of Applied Psychology, 99: 535-545.

Avery, D. R., McKay, P. F. \& Volpone, S. D. 2016. Blaming the building: How venue quality influences consumer bias against stigmatized leaders. Journal of Applied Psychology, 101: 1111-1121.

Ballard, T., Yeo, G., Loft, S., Vancouver, J. B. \& Neal, A. 2016. An integrative formal model of motivation and decision making: The mgpm*. Journal of Applied Psychology, 101: 12401265.

Ballinger, G. A., Cross, R. \& Holtom, B. C. 2016. The right friends in the right places: Understanding network structure as a predictor of voluntary turnover. Journal of Applied Psychology, 101: 535-548.

Barnes, C. M., Reb, J. \& Ang, D. 2012. More than just the mean: Moving to a dynamic view of performance-based compensation. Journal of Applied Psychology, 97: 711-718.

Beatty, A. S., Barratt, C. L., Berry, C. M. \& Sackett, P. R. 2014. Testing the generalizability of indirect range restriction corrections. Journal of Applied Psychology, 99: 587-598.

Beck, J. W. \& Schmidt, A. M. 2013. State-level goal orientations as mediators of the relationship between time pressure and performance: A longitudinal study. Journal of Applied Psychology, 98: 354-363.

Beck, J. W., Scholer, A. A. \& Hughes, J. 2017. Divergent effects of distance versus velocity disturbances on emotional experiences during goal pursuit. Journal of Applied Psychology, 102: 1109-1123.

Belmi, P. \& Pfeffer, J. 2016. Power and death: Mortality salience increases power seeking while feeling powerful reduces death anxiety. Journal of Applied Psychology, 101: 702-720. 
Bennett, A. A., Gabriel, A. S., Calderwood, C., Dahling, J. J. \& Trougakos, J. P. 2016. Better together? Examining profiles of employee recovery experiences. Journal of Applied Psychology, 101: 1635-1654.

Berry, C. M., Cullen, M. J. \& Meyer, J. M. 2014. Racial/ethnic subgroup differences in cognitive ability test range restriction: Implications for differential validity. Journal of Applied Psychology, 99: 21-37.

Bezrukova, K., Spell, C. S., Caldwell, D. \& Burger, J. M. 2016. A multilevel perspective on faultlines: Differentiating the effects between group- and organizational-level faultlines. Journal of Applied Psychology, 101: 86-107.

Bindl, U. K., Parker, S. K., Totterdell, P. \& Hagger-Johnson, G. 2012. Fuel of the self-starter: How mood relates to proactive goal regulation. Journal of Applied Psychology, 97: 134150.

Biron, M. \& Bamberger, P. 2012. Aversive workplace conditions and absenteeism: Taking referent group norms and supervisor support into account. Journal of Applied Psychology, 97: 901-912.

Blanton, H., Jaccard, J., Strauts, E., Mitchell, G. \& Tetlock, P. E. 2015. Toward a meaningful metric of implicit prejudice. Journal of Applied Psychology, 100: 1468-1481.

Bonner, J. M., Greenbaum, R. L. \& Quade, M. J. 2017. Employee unethical behavior to shame as an indicator of self-image threat and exemplification as a form of self-image protection: The exacerbating role of supervisor bottom-line mentality. Journal of Applied Psychology, 102: 1203-1221.

Brooks, M. E., Dalal, D. K. \& Nolan, K. P. 2014. Are common language effect sizes easier to understand than traditional effect sizes? Journal of Applied Psychology, 99: 332-340. 
Brosi, P., Sporrle, M., Welpe, I. M. \& Heilman, M. E. 2016. Expressing pride: Effects on perceived agency, communality, and stereotype-based gender disparities. Journal of Applied Psychology, 101: 1319-1328.

Byrne, Z. S., Peters, J. M. \& Weston, J. W. 2016. The struggle with employee engagement: Measures and construct clarification using five samples. Journal of Applied Psychology, 101: 1201-1227.

Caleo, S. 2016. Are organizational justice rules gendered? Reactions to men's and women's justice violations. Journal of Applied Psychology, 101: 1422-1435.

Campagna, R. L., Mislin, A. A., Kong, D. T. \& Bottom, W. P. 2016. Strategic consequences of emotional misrepresentation in negotiation: The blowback effect. Journal of Applied Psychology, 101: 605-624.

Campbell, E. M., Liao, H., Chuang, A., Zhou, J. \& Dong, Y. 2017. Hot shots and cool reception? An expanded view of social consequences for high performers. Journal of Applied Psychology, 102: 845-866.

Carter, N. T., Dalal, D. K., Boyce, A. S., O'Connell, M. S., Kung, M.-C. \& Delgado, K. M. 2014. Uncovering curvilinear relationships between conscientiousness and job performance: How theoretically appropriate measurement makes an empirical difference. Journal of Applied Psychology, 99: 564-586.

Chen, M., Chen, C. C. \& Sheldon, O. J. 2016. Relaxing moral reasoning to win: How organizational identification relates to unethical pro-organizational behavior. Journal of Applied Psychology, 101: 1082-1096.

Chen, P., Myers, C. G., Kopelman, S. \& Garcia, S. M. 2012. The hierarchical face: Higher rankings lead to less cooperative looks. Journal of Applied Psychology, 97: 479-486. 
Chi, W., Li, W.-D., Wang, N. \& Song, Z. 2016. Can genes play a role in explaining frequent job changes? An examination of gene-environment interaction from human capital theory. Journal of Applied Psychology, 101: 1030-1044.

da Motta Veiga, S. P. \& Gabriel, A. S. 2016. The role of self-determined motivation in job search: A dynamic approach. Journal of Applied Psychology, 101: 350-361.

Dang, C. T., Umphress, E. E. \& Mitchell, M. S. 2017. Leader social accounts of subordinates' unethical behavior: Examining observer reactions to leader social accounts with moral disengagement language. Journal of Applied Psychology, 102: 1448-1461.

de Wit, F. R. C., Greer, L. L. \& Jehn, K. A. 2012. The paradox of intragroup conflict: A metaanalysis. Journal of Applied Psychology, 97: 360-390.

DeCelles, K. A., DeRue, D. S., Margolis, J. D. \& Ceranic, T. L. 2012. Does power corrupt or enable? When and why power facilitates self-interested behavior. Journal of Applied Psychology, 97: 681-689.

Den Hartog, D. N. \& Belschak, F. D. 2012. When does transformational leadership enhance employee proactive behavior? The role of autonomy and role breadth self-efficacy. Journal of Applied Psychology, 97: 194-202.

Diestel, S., Rivkin, W. \& Schmidt, K.-H. 2015. Sleep quality and self-control capacity as protective resources in the daily emotional labor process: Results from two diary studies. Journal of Applied Psychology, 100: 809-827.

Dietz, B., van Knippenberg, D., Hirst, G. \& Restubog, S. L. D. 2015. Outperforming whom? A multilevel study of performance-prove goal orientation, performance, and the moderating role of shared team identification. Journal of Applied Psychology, 100: 1811-1824. 
Dimotakis, N., Conlon, D. E. \& Ilies, R. 2012. The mind and heart (literally) of the negotiator: Personality and contextual determinants of experiential reactions and economic outcomes in negotiation. Journal of Applied Psychology, 97: 183-193.

Dragoni, L. \& Kuenzi, M. 2012. Better understanding work unit goal orientation: Its emergence and impact under different types of work unit structure. Journal of Applied Psychology, 97: 1032-1048.

Dragoni, L., Park, H., Soltis, J. \& Forte-Trammell, S. 2014. Show and tell: How supervisors facilitate leader development among transitioning leaders. Journal of Applied Psychology, 99: 66-86.

Duguid, M. M. \& Thomas-Hunt, M. C. 2015. Condoning stereotyping? How awareness of stereotyping prevalence impacts expression of stereotypes. Journal of Applied Psychology, 100: 343-359.

Eberly, M. B., Holley, E. C., Johnson, M. D. \& Mitchell, T. R. 2017. It's not me, it's not you, it's us! An empirical examination of relational attributions. Journal of Applied Psychology, 102: 711-731.

Eby, L. T., Durley, J. R., Evans, S. C. \& Ragins, B. R. 2008. Mentors' perceptions of negative mentoring experiences: Scale development and nomological validation. Journal of Applied Psychology, 93: 358-373.

Ellingson, J. E., Tews, M. J. \& Dachner, A. M. 2016. Constituent attachment and voluntary turnover in low-wage/low-skill service work. Journal of Applied Psychology, 101: 129140. 
Ellington, K. J., Dierdorff, E. C. \& Rubin, R. S. 2014. Decelerating the diminishing returns of citizenship on task performance: The role of social context and interpersonal skill. Journal of Applied Psychology, 99: 748-758.

Ellis, A. P. J., Mai, K. M. \& Christian, J. S. 2013. Examining the asymmetrical effects of goal faultlines in groups: A categorization-elaboration approach. Journal of Applied Psychology, 98: 948-961.

Farh, C. I. C. \& Chen, Z. 2014. Beyond the individual victim: Multilevel consequences of abusive supervision in teams. Journal of Applied Psychology, 99: 1074-1095.

Farh, C. I. C., Seo, M.-G. \& Tesluk, P. E. 2012. Emotional intelligence, teamwork effectiveness, and job performance: The moderating role of job context. Journal of Applied Psychology, 97: 890-900.

Ferguson, A. J. \& Peterson, R. S. 2015. Sinking slowly: Diversity in propensity to trust predicts downward trust spirals in small groups. Journal of Applied Psychology, 100: 1012-1024.

Ferris, D. L., Brown, D. J., Berry, J. W. \& Lian, H. 2008. The development and validation of the workplace ostracism scale. 1348. United States: APA AMERICAN PSYCHOLOGICAL ASSOCIATION.

Ferris, D. L., Johnson, R. E., Rosen, C. C., Djurdjevic, E., Chang, C.-H. \& Tan, J. A. 2013. When is success not satisfying? Integrating regulatory focus and approach/avoidance motivation theories to explain the relation between core self-evaluation and job satisfaction. Journal of Applied Psychology, 98: 342-353.

Fine, S., Goldenberg, J. \& Noam, Y. 2016. Beware of those left behind: Counterproductive work behaviors among nonpromoted employees and the moderating effect of integrity. Journal of Applied Psychology, 101: 1721-1729. 
Fisher, D. M. 2014. Distinguishing between taskwork and teamwork planning in teams: Relations with coordination and interpersonal processes. Journal of Applied Psychology, 99: 423-436.

Fisher, D. M. 2014. A multilevel cross-cultural examination of role overload and organizational commitment: Investigating the interactive effects of context. Journal of Applied Psychology, 99: 723-736.

Fisher, D. M., Bell, S. T., Dierdorff, E. C. \& Belohlav, J. A. 2012. Facet personality and surfacelevel diversity as team mental model antecedents: Implications for implicit coordination. Journal of Applied Psychology, 97: 825-841.

Foulk, T. A. \& Long, D. M. 2016. Impressed by impression management: Newcomer reactions to ingratiated supervisors. Journal of Applied Psychology, 101: 1487-1497.

Gabriel, A. S., Daniels, M. A., Diefendorff, J. M. \& Greguras, G. J. 2015. Emotional labor actors: A latent profile analysis of emotional labor strategies. Journal of Applied Psychology, 100: 863-879.

Garcia, P. R. J. M., Restubog, S. L. D., Kiewitz, C., Scott, K. L. \& Tang, R. L. 2014. Roots run deep: Investigating psychological mechanisms between history of family aggression and abusive supervision. Journal of Applied Psychology, 99: 883-897.

Gelfand, M. J., Brett, J., Gunia, B. C., Imai, L., Huang, T.-J. \& Hsu, B.-F. 2013. Toward a culture-by-context perspective on negotiation: Negotiating teams in the united states and taiwan. Journal of Applied Psychology, 98: 504-513.

Gerbasi, A., Porath, C. L., Parker, A., Spreitzer, G. \& Cross, R. 2015. Destructive de-energizing relationships: How thriving buffers their effect on performance. Journal of Applied Psychology, 100: 1423-1433. 
Giessner, S. R., van Knippenberg, D., van Ginkel, W. \& Sleebos, E. 2013. Team-oriented leadership: The interactive effects of leader group prototypicality, accountability, and team identification. Journal of Applied Psychology, 98: 658-667.

Giumetti, G. W., Schroeder, A. N. \& Switzer, F. S., III. 2015. Forced distribution rating systems: When does "rank and yank" lead to adverse impact? Journal of Applied Psychology, 100: 180-193.

Grand, J. A., Braun, M. T., Kuljanin, G., Kozlowski, S. W. J. \& Chao, G. T. 2016. The dynamics of team cognition: A process-oriented theory of knowledge emergence in teams. Journal of Applied Psychology, 101: 1353-1385.

Grant, A. M. \& Rothbard, N. P. 2013. When in doubt, seize the day? Security values, prosocial values, and proactivity under ambiguity. Journal of Applied Psychology, 98: 810-819.

Greenbaum, R. L., Quade, M. J., Mawritz, M. B., Kim, J. \& Crosby, D. 2014. When the customer is unethical: The explanatory role of employee emotional exhaustion onto work-family conflict, relationship conflict with coworkers, and job neglect. Journal of Applied Psychology, 99: 1188-1203.

Guarana, C. L. \& Hernandez, M. 2016. Identified ambivalence: When cognitive conflicts can help individuals overcome cognitive traps. Journal of Applied Psychology, 101: 10131029.

Gunia, B. C., Brett, J. M., Nandkeolyar, A. K. \& Kamdar, D. 2011. Paying a price: Culture, trust, and negotiation consequences. Journal of Applied Psychology, 96: 774-789.

Gupta, N., Ganster, D. C. \& Kepes, S. 2013. Assessing the validity of sales self-efficacy: A cautionary tale. Journal of Applied Psychology, 98: 690-700. 
Hahn, V. C. \& Dormann, C. 2013. The role of partners and children for employees' psychological detachment from work and well-being. Journal of Applied Psychology, 98: $26-36$.

Hanek, K. J., Garcia, S. M. \& Tor, A. 2016. Gender and competitive preferences: The role of competition size. Journal of Applied Psychology, 101: 1122-1133.

Harman, R. P., Ellington, K. J., Surface, E. A. \& Thompson, L. F. 2015. Exploring qualitative training reactions: Individual and contextual influences on trainee commenting. Journal of Applied Psychology, 100: 894-916.

Harold, C. M., Oh, I.-S., Holtz, B. C., Han, S. \& Giacalone, R. A. 2016. Fit and frustration as drivers of targeted counterproductive work behaviors: A multifoci perspective. Journal of Applied Psychology, 101: 1513-1535.

Hernandez, M., Avery, D. R., Tonidandel, S., Hebl, M. R., Smith, A. N. \& McKay, P. F. 2016. The role of proximal social contexts: Assessing stigma-by-association effects on leader appraisals. Journal of Applied Psychology, 101: 68-85.

Hershcovis, M. S. \& Bhatnagar, N. 2017. When fellow customers behave badly: Witness reactions to employee mistreatment by customers. Journal of Applied Psychology, 102: $1528-1544$.

Hideg, I. \& Ferris, D. L. 2017. Dialectical thinking and fairness-based perspectives of affirmative action. Journal of Applied Psychology, 102: 782-801.

Hillebrandt, A. \& Barclay, L. J. 2017. Comparing integral and incidental emotions: Testing insights from emotions as social information theory and attribution theory. Journal of Applied Psychology, 102: 732-752. 
Hochwarter, W. A., Ferris, G. R., Zinko, R., Arnell, B. \& James, M. 2007. Reputation as a moderator of political behavior-work outcomes relationships: A two-study investigation with convergent results. Journal of Applied Psychology, 92: 567-576.

Huang, G.-H., Wellman, N., Ashford, S. J., Lee, C. \& Wang, L. 2017. Deviance and exit: The organizational costs of job insecurity and moral disengagement. Journal of Applied Psychology, 102: 26-42.

Huang, J. L., Liu, M. \& Bowling, N. A. 2015. Insufficient effort responding: Examining an insidious confound in survey data. Journal of Applied Psychology, 100: 828-845.

Huang, X., Hsieh, P.-A. J. J. \& He, W. 2014. Expertise dissimilarity and creativity: The contingent roles of tacit and explicit knowledge sharing. Journal of Applied Psychology, 99: 816-830.

Hulsheger, U. R. 2016. From dawn till dusk: Shedding light on the recovery process by investigating daily change patterns in fatigue. Journal of Applied Psychology, 101: 905914.

Hulsheger, U. R., Alberts, H. J., Feinholdt, A. \& Lang, J. W. 2013. Benefits of mindfulness at work: The role of mindfulness in emotion regulation, emotional exhaustion, and job satisfaction. Journal of Applied Psychology, 98: 310-325.

Hulsheger, U. R., Lang, J. W. B., Schewe, A. F. \& Zijlstra, F. R. H. 2015. When regulating emotions at work pays off: A diary and an intervention study on emotion regulation and customer tips in service jobs. Journal of Applied Psychology, 100: 263-277.

Jiang, L. \& Probst, T. M. 2017. The rich get richer and the poor get poorer: Country- and statelevel income inequality moderates the job insecurity-burnout relationship. Journal of Applied Psychology, 102: 672-681. 
Johnson, M. D., Ilies, R. \& Boles, T. L. 2012. Alternative reference points and outcome evaluation: The influence of affect. Journal of Applied Psychology, 97: 33-45.

Joseph, D. L., Jin, J., Newman, D. A. \& O'Boyle, E. H. 2015. Why does self-reported emotional intelligence predict job performance? A meta-analytic investigation of mixed ei. Journal of Applied Psychology, 100: 298-342.

Judge, T. A., Rodell, J. B., Klinger, R. L., Simon, L. S. \& Crawford, E. R. 2013. Hierarchical representations of the five-factor model of personality in predicting job performance: Integrating three organizing frameworks with two theoretical perspectives. Journal of Applied Psychology, 98: 875-925.

Kark, R., Katz-Navon, T. \& Delegach, M. 2015. The dual effects of leading for safety: The mediating role of employee regulatory focus. Journal of Applied Psychology, 100: 13321348.

Keiser, H. N., Sackett, P. R., Kuncel, N. R. \& Brothen, T. 2016. Why women perform better in college than admission scores would predict: Exploring the roles of conscientiousness and course-taking patterns. Journal of Applied Psychology, 101: 569-581.

Kiewitz, C., Restubog, S. L. D., Shoss, M. K., Garcia, P. R. J. M. \& Tang, R. L. 2016. Suffering in silence: Investigating the role of fear in the relationship between abusive supervision and defensive silence. Journal of Applied Psychology, 101: 731-742.

Kinias, Z. \& Sim, J. 2016. Facilitating women's success in business: Interrupting the process of stereotype threat through affirmation of personal values. Journal of Applied Psychology, 101: 1585 . 
Klein, R. M., Dilchert, S., Ones, D. S. \& Dages, K. D. 2015. Cognitive predictors and age-based adverse impact among business executives. Journal of Applied Psychology, 100: 14971510.

Kluemper, D. H., McLarty, B. D. \& Bing, M. N. 2015. Acquaintance ratings of the big five personality traits: Incremental validity beyond and interactive effects with self-reports in the prediction of workplace deviance: Research report. Journal of Applied Psychology, 100: 237-248.

Kouchaki, M. \& Desai, S. D. 2015. Anxious, threatened, and also unethical: How anxiety makes individuals feel threatened and commit unethical acts. Journal of Applied Psychology, 100: $360-375$.

Krumm, S., Lievens, F., Huffmeier, J., Lipnevich, A. A., Bendels, H. \& Hertel, G. 2015. How "situational" is judgment in situational judgment tests? Journal of Applied Psychology, 100: 399-416.

Kwong, J. Y. Y. \& Fai Ellick Wong, K. 2014. Reducing and exaggerating escalation of commitment by option partitioning. Journal of Applied Psychology, 99: 697-712.

Lacerenza, C. N., Reyes, D. L., Marlow, S. L., Joseph, D. L. \& Salas, E. 2017. Leadership training design, delivery, and implementation: A meta-analysis. Journal of Applied Psychology, 102: 1686-1718.

Lambert, L. S., Tepper, B. J., Carr, J. C., Holt, D. T. \& Barelka, A. J. 2012. Forgotten but not gone: An examination of fit between leader consideration and initiating structure needed and received. Journal of Applied Psychology, 97: 913-930. 
Lanaj, K., Johnson, R. E. \& Lee, S. M. 2016. Benefits of transformational behaviors for leaders: A daily investigation of leader behaviors and need fulfillment. Journal of Applied Psychology, 101: 237-251.

Le, H., Robbins, S. B. \& Westrick, P. 2014. Predicting student enrollment and persistence in college stem fields using an expanded p-e fit framework: A large-scale multilevel study. Journal of Applied Psychology, 99: 915-947.

Lee, J. J., Gino, F. \& Staats, B. R. 2014. Rainmakers: Why bad weather means good productivity: Research report. Journal of Applied Psychology, 99: 504-513.

Leroy, H., Dierynck, B., Anseel, F., Simons, T., Halbesleben, J. R., McCaughey, D., Savage, G. T. \& Sels, L. 2012. Behavioral integrity for safety, priority of safety, psychological safety, and patient safety: A team-level study. Journal of Applied Psychology, 97: 12731281.

Levi, A. S. \& Fried, Y. 2008. Differences between african americans and whites in reactions to affirmative action programs in hiring, promotion, training, and layoffs. Journal of Applied Psychology, 93: 1118-1129.

Li, J., Lee, T. W., Mitchell, T. R., Hom, P. W. \& Griffeth, R. W. 2016. The effects of proximal withdrawal states on job attitudes, job searching, intent to leave, and employee turnover. Journal of Applied Psychology, 101: 1436-1456.

Lian, H., Ferris, D. L. \& Brown, D. J. 2012. Does power distance exacerbate or mitigate the effects of abusive supervision? It depends on the outcome. Journal of Applied Psychology, 97: 107-123. 
Lievens, F., Schollaert, E. \& Keen, G. 2015. The interplay of elicitation and evaluation of traitexpressive behavior: Evidence in assessment center exercises. Journal of Applied Psychology, 100: 1169-1188.

Lin, S.-H. \& Johnson, R. E. 2015. A suggestion to improve a day keeps your depletion away: Examining promotive and prohibitive voice behaviors within a regulatory focus and ego depletion framework. Journal of Applied Psychology, 100: 1381-1397.

Lin, S.-H., Ma, J. \& Johnson, R. E. 2016. When ethical leader behavior breaks bad: How ethical leader behavior can turn abusive via ego depletion and moral licensing. Journal of Applied Psychology, 101: 815-830.

Liu, W., Gong, Y. \& Liu, J. 2014. When do business units benefit more from collective citizenship behavior of management teams? An upper echelons perspective: Research report. Journal of Applied Psychology, 99: 523-534.

Liu, W., Tangirala, S., Lam, W., Chen, Z., Jia, R. T. \& Huang, X. 2015. How and when peers' positive mood influences employees' voice. Journal of Applied Psychology, 100: 976989.

Lindsey, A. P., Avery, D. R., Dawson, J. F. \& King, E. B. 2017. Investigating why and for whom management ethnic representativeness influences interpersonal mistreatment in the workplace. Journal of Applied Psychology, 102: 1545-1563.

Long, E. C. \& Christian, M. S. 2015. Mindfulness buffers retaliatory responses to injustice: A regulatory approach. Journal of Applied Psychology, 100: 1409-1422.

Loschelder, D. D., Trotschel, R., Swaab, R. I., Friese, M. \& Galinsky, A. D. 2016. The information-anchoring model of first offers: When moving first helps versus hurts negotiators. Journal of Applied Psychology, 101: 995-1012. 
Luchak, A. A. \& Gellatly, I. R. 2007. A comparison of linear and nonlinear relations between organizational commitment and work outcomes. Journal of Applied Psychology, 92: 786793.

Luksyte, A., Avery, D. R. \& Yeo, G. 2015. It is worse when you do it: Examining the interactive effects of coworker presenteeism and demographic similarity. Journal of Applied Psychology, 100: 1107-1123.

Lyons, B. J., Volpone, S. D., Wessel, J. L. \& Alonso, N. M. 2017. Disclosing a disability: Do strategy type and onset controllability make a difference? Journal of Applied Psychology, 102: $1375-1383$.

Madera, J. M. \& Hebl, M. R. 2012. Discrimination against facially stigmatized applicants in interviews: An eye-tracking and face-to-face investigation. Journal of Applied Psychology, 97: 317-330.

Madrid, H. P., Totterdell, P., Niven, K. \& Barros, E. 2016. Leader affective presence and innovation in teams. Journal of Applied Psychology, 101: 673-686.

Mai, K. M., Ellis, A. P. J., Christian, J. S. \& Porter, C. O. L. H. 2016. Examining the effects of turnover intentions on organizational citizenship behaviors and deviance behaviors: A psychological contract approach. Journal of Applied Psychology, 101: 1067-1081.

Martinez, L. R., Sawyer, K. B., Thoroughgood, C. N., Ruggs, E. N. \& Smith, N. A. 2017. The importance of being "me": The relation between authentic identity expression and transgender employees' work-related attitudes and experiences. Journal of Applied Psychology, 102: 215-226. 
Martinez, L. R., White, C. D., Shapiro, J. R. \& Hebl, M. R. 2016. Selection bias: Stereotypes and discrimination related to having a history of cancer. Journal of Applied Psychology, 101: $122-128$.

Mathieu, J. E., Kukenberger, M. R., D'Innocenzo, L. \& Reilly, G. 2015. Modeling reciprocal team cohesion-performance relationships, as impacted by shared leadership and members' competence. Journal of Applied Psychology, 100: 713-734.

Maynard, T. M., Luciano, M. M., D'Innocenzo, L., Mathieu, J. E. \& Dean, M. D. 2014. Modeling time-lagged reciprocal psychological empowerment-performance relationships. Journal of Applied Psychology, 99: 1244-1253.

McCarthy, J. M., Trougakos, J. P. \& Cheng, B. H. 2016. Are anxious workers less productive workers? It depends on the quality of social exchange. Journal of Applied Psychology, 101: 279-291.

McGonagle, A. K., Fisher, G. G., Barnes-Farrell, J. L. \& Grosch, J. W. 2015. Individual and work factors related to perceived work ability and labor force outcomes. Journal of Applied Psychology, 100: 376-398.

Melwani, S., Mueller, J. S. \& Overbeck, J. R. 2012. Looking down: The influence of contempt and compassion on emergent leadership categorizations. Journal of Applied Psychology, 97: 1171-1185.

Mitchell, M. S. \& Ambrose, M. L. 2012. Employees' behavioral reactions to supervisor aggression: An examination of individual and situational factors. Journal of Applied Psychology, 97: 1148-1170. 
Nahum-Shani, I., Henderson, M. M., Lim, S. \& Vinokur, A. D. 2014. Supervisor support: Does supervisor support buffer or exacerbate the adverse effects of supervisor undermining? Journal of Applied Psychology, 99: 484-503.

Naveh, E. \& Katz-Navon, T. 2015. A longitudinal study of an intervention to improve road safety climate: Climate as an organizational boundary spanner: Research report. Journal of Applied Psychology, 100: 216-226.

Niessen, C. \& Jimmieson, N. L. 2016. Threat of resource loss: The role of self-regulation in adaptive task performance. Journal of Applied Psychology, 101: 450-462.

Nye, C. D., Brummel, B. J. \& Drasgow, F. 2014. Understanding sexual harassment using aggregate construct models. Journal of Applied Psychology, 99: 1204-1221.

Oc, B., Bashshur, M. R. \& Moore, C. 2015. Speaking truth to power: The effect of candid feedback on how individuals with power allocate resources. Journal of Applied Psychology, 100: 450-463.

O'Reilly, J., Aquino, K. \& Skarlicki, D. 2016. The lives of others: Third parties' responses to others' injustice. Journal of Applied Psychology, 101: 171-189.

Owens, B. P., Baker, W. E., Sumpter, D. M. \& Cameron, K. S. 2016. Relational energy at work: Implications for job engagement and job performance. Journal of Applied Psychology, 101: 35-49.

Pearsall, M. J. \& Venkataramani, V. 2015. Overcoming asymmetric goals in teams: The interactive roles of team learning orientation and team identification. Journal of Applied Psychology, 100: 735-748. 
Perry-Smith, J. E. 2014. Social network ties beyond nonredundancy: An experimental investigation of the effect of knowledge content and tie strength on creativity. Journal of Applied Psychology, 99: 831-846.

Pitesa, M. \& Thau, S. 2013. Masters of the universe: How power and accountability influence self-serving decisions under moral hazard. Journal of Applied Psychology, 98: 550-558.

Porath, C. L., Gerbasi, A. \& Schorch, S. L. 2015. The effects of civility on advice, leadership, and performance. Journal of Applied Psychology, 100: 1527-1541.

Qin, X., Ren, R., Zhang, Z.-X. \& Johnson, R. E. 2015. Fairness heuristics and substitutability effects: Inferring the fairness of outcomes, procedures, and interpersonal treatment when employees lack clear information. Journal of Applied Psychology, 100: 749-766.

Rafaeli, A., Erez, A., Ravid, S., Derfler-Rozin, R., Treister, D. E. \& Scheyer, R. 2012. When customers exhibit verbal aggression, employees pay cognitive costs. Journal of Applied Psychology, 97: 931-950.

Reb, J. \& Greguras, G. J. 2010. Understanding performance ratings: Dynamic performance, attributions, and rating purpose. Journal of Applied Psychology, 95: 213.

Reich, T. C. \& Hershcovis, S. M. 2015. Observing workplace incivility: Research report. Journal of Applied Psychology, 100: 203-215.

Ritter, K.-J., Matthews, R. A., Ford, M. T. \& Henderson, A. A. 2016. Understanding role stressors and job satisfaction over time using adaptation theory. Journal of Applied Psychology, 101: 1655-1669.

Rockmann, K. W. \& Ballinger, G. A. 2017. Intrinsic motivation and organizational identification among on-demand workers. Journal of Applied Psychology, 102: 1305-1316. 
Rockstuhl, T., Ang, S., Ng, K.-Y., Lievens, F. \& Van Dyne, L. 2015. Putting judging situations into situational judgment tests: Evidence from intercultural multimedia sjts. Journal of Applied Psychology, 100: 464-480.

Ryan, A. M. \& Nguyen, H.-H. D. 2017. Publication bias and stereotype threat research: A reply to Zigerell. Journal of Applied Psychology, 102: 1169-1177.

Schaumberg, R. L. \& Flynn, F. J. 2017. Clarifying the link between job satisfaction and absenteeism: The role of guilt proneness. Journal of Applied Psychology, 102: 982-992.

Schleicher, D. J., Smith, T. A., Casper, W. J., Watt, J. D. \& Greguras, G. J. 2015. It's all in the attitude: The role of job attitude strength in job attitude-outcome relationships. Journal of Applied Psychology, 100: 1259-1274.

Schultze, T., Pfeiffer, F. \& Schulz-Hardt, S. 2012. Biased information processing in the escalation paradigm: Information search and information evaluation as potential mediators of escalating commitment. Journal of Applied Psychology, 97: 16-32.

Scott, K. L., Restubog, S. L. \& Zagenczyk, T. J. 2013. A social exchange-based model of the antecedents of workplace exclusion. Journal of Applied Psychology, 98: 37-48.

Sheldon, O. J., Dunning, D. \& Ames, D. R. 2014. Emotionally unskilled, unaware, and uninterested in learning more: Reactions to feedback about deficits in emotional intelligence. Journal of Applied Psychology, 99: 125-137.

Shoss, M. K., Eisenberger, R., Restubog, S. L. \& Zagenczyk, T. J. 2013. Blaming the organization for abusive supervision: The roles of perceived organizational support and supervisor's organizational embodiment. Journal of Applied Psychology, 98: 158-168. 
Sinaceur, M., Van Kleef, G. A., Neale, M. A., Adam, H. \& Haag, C. 2011. Hot or cold: Is communicating anger or threats more effective in negotiation? Journal of Applied Psychology, 96: 1018.

Sinha, R., Janardhanan, N. S., Greer, L. L., Conlon, D. E. \& Edwards, J. R. 2016. Skewed task conflicts in teams: What happens when a few members see more conflict than the rest? Journal of Applied Psychology, 101: 1045-1055.

Skarlicki, D. P., van Jaarsveld, D. D., Shao, R., Song, Y. H. \& Wang, M. 2016. Extending the multifoci perspective: The role of supervisor justice and moral identity in the relationship between customer justice and customer-directed sabotage. Journal of Applied Psychology, 101: 108-121.

Sliter, M. T., Sinclair, R. R., Yuan, Z. \& Mohr, C. D. 2014. Don't fear the reaper: Trait death anxiety, mortality salience, and occupational health. Journal of Applied Psychology, 99: $759-769$.

Sonnentag, S., Mojza, E. J., Demerouti, E. \& Bakker, A. B. 2012. Reciprocal relations between recovery and work engagement: The moderating role of job stressors. Journal of Applied Psychology, 97: 842-853.

Speer, A. B., Christiansen, N. D., Goffin, R. D. \& Goff, M. 2014. Situational bandwidth and the criterion-related validity of assessment center ratings: Is cross-exercise convergence always desirable? Journal of Applied Psychology, 99: 282-295.

Spieler, I., Scheibe, S., Stamov-Ronagel, C. \& Kappas, A. 2017. Help or hindrance? Day-level relationships between flextime use, work-nonwork boundaries, and affective well-being. Journal of Applied Psychology, 102: 67-87. 
Stoverink, A. C., Umphress, E. E., Gardner, R. G. \& Miner, K. N. 2014. Misery loves company: Team dissonance and the influence of supervisor-focused interpersonal justice climate on team cohesiveness. Journal of Applied Psychology, 99: 1059-1073.

Strauss, K., Griffin, M. A. \& Parker, S. K. 2012. Future work selves: How salient hoped-for identities motivate proactive career behaviors. Journal of Applied Psychology, 97: 580598.

Sturman, M. C., Shao, L. \& Katz, J. H. 2012. The effect of culture on the curvilinear relationship between performance and turnover. Journal of Applied Psychology, 97: 46-62.

Sun, S., Song, Z. \& Lim, V. K. 2013. Dynamics of the job search process: Developing and testing a mediated moderation model. Journal of Applied Psychology, 98: 771-784.

Thau, S., Derfler-Rozin, R., Pitesa, M., Mitchell, M. S. \& Pillutla, M. M. 2015. Unethical for the sake of the group: Risk of social exclusion and pro-group unethical behavior. Journal of Applied Psychology, 100: 98-113.

Unsworth, K. L. \& McNeill, I. M. 2017. Increasing pro-environmental behaviors by increasing self-concordance: Testing an intervention. Journal of Applied Psychology, 102: 88-103.

van Hooft, E. A. J. \& Born, M. P. 2012. Intentional response distortion on personality tests: Using eye-tracking to understand response processes when faking. Journal of Applied Psychology, 97: 301-316.

Van Iddekinge, C. H., Roth, P. L., Raymark, P. H. \& Odle-Dusseau, H. N. 2012. The criterionrelated validity of integrity tests: An updated meta-analysis. Journal of Applied Psychology, 97: 499-530. 
Van Kleef, G. A., Steinel, W. \& Homan, A. C. 2013. On being peripheral and paying attention: Prototypicality and information processing in intergroup conflict. Journal of Applied Psychology, 98: 63-79.

Van Kleef, G. A., van den Berg, H. \& Heerdink, M. W. 2015. The persuasive power of emotions: Effects of emotional expressions on attitude formation and change. Journal of Applied Psychology, 100: 1124-1142.

Venkataramani, V., Labianca, G. \& Grosser, T. 2013. Positive and negative workplace relationships, social satisfaction, and organizational attachment. Journal of Applied Psychology, 98: 1028-1039.

Venkataramani, V., Richter, A. W. \& Clarke, R. 2014. Creative benefits from well-connected leaders: Leader social network ties as facilitators of employee radical creativity. Journal of Applied Psychology, 99: 966-975.

Walker, D. D., van Jaarsveld, D. D. \& Skarlicki, D. P. 2017. Sticks and stones can break my bones but words can also hurt me: The relationship between customer verbal aggression and employee incivility. Journal of Applied Psychology, 102: 163-179.

Walker, H. J., Feild, H. S., Bernerth, J. B. \& Becton, J. B. 2012. Diversity cues on recruitment websites: Investigating the effects on job seekers' information processing. Journal of Applied Psychology, 97: 214-224.

Walter, F., Lam, C. K., van der Vegt, G. S., Huang, X. \& Miao, Q. 2015. Abusive supervision and subordinate performance: Instrumentality considerations in the emergence and consequences of abusive supervision. Journal of Applied Psychology, 100: 1056-1072.

Wang, D., Waldman, D. A. \& Zhang, Z. 2014. A meta-analysis of shared leadership and team effectiveness. Journal of Applied Psychology, 99: 181-198. 
Wang, H.-J., Lu, C.-Q. \& Siu, O.-L. 2015. Job insecurity and job performance: The moderating role of organizational justice and the mediating role of work engagement. Journal of Applied Psychology, 100: 1249-1258.

Wang, L. \& Murnighan, J. K. 2017. The dynamics of punishment and trust. Journal of Applied Psychology, 102: 1385-1402.

Wang, M., Liu, S., Liao, H., Gong, Y., Kammeyer-Mueller, J. \& Shi, J. c. 2013. Can't get it out of my mind: Employee rumination after customer mistreatment and negative mood in the next morning. Journal of Applied Psychology, 98: 989-1004.

Ward, A.-K., Ravlin, E. C., Klaas, B. S., Ployhart, R. E. \& Buchan, N. R. 2016. When do highcontext communicators speak up? Exploring contextual communication orientation and employee voice. Journal of Applied Psychology, 101: 1498-1511.

Wegge, J., Roth, C., Neubach, B., Schmidt, K.-H. \& Kanfer, R. 2008. Age and gender diversity as determinants of performance and health in a public organization: The role of task complexity and group size. Journal of Applied Psychology, 93: 1301-1313.

Wellman, N., Mayer, D. M., Ong, M. \& DeRue, D. S. 2016. When are do-gooders treated badly? Legitimate power, role expectations, and reactions to moral objection in organizations. Journal of Applied Psychology, 101: 793-814.

Whiting, S. W., Maynes, T. D., Podsakoff, N. P. \& Podsakoff, P. M. 2012. Effects of message, source, and context on evaluations of employee voice behavior. Journal of Applied Psychology, 97: 159-182.

Williams, L. J. \& O'Boyle, E. H. 2015. Ideal, nonideal, and no-marker variables: The confirmatory factor analysis (cfa) marker technique works when it matters. Journal of Applied Psychology, 100: 1579-1602. 
Winterich, K. P., Aquino, K., Mittal, V. \& Swartz, R. 2013. When moral identity symbolization motivates prosocial behavior: The role of recognition and moral identity internalization. Journal of Applied Psychology, 98: 759-770.

Wu, C.-H. \& Griffin, M. A. 2012. Longitudinal relationships between core self-evaluations and job satisfaction. Journal of Applied Psychology, 97: 331-342.

Wu, C.-H., Liu, J., Kwan, H. K. \& Lee, C. 2016. Why and when workplace ostracism inhibits organizational citizenship behaviors: An organizational identification perspective. Journal of Applied Psychology, 101: 362-378.

Yam, K. C., Fehr, R. \& Barnes, C. M. 2014. Morning employees are perceived as better employees: Employees' start times influence supervisor performance ratings. Journal of Applied Psychology, 99: 1288-1299.

Yu, J., Downes, P. E., Carter, K. M. \& O'Boyle, E. H. 2016. The problem of effect size heterogeneity in meta-analytic structural equation modeling. Journal of Applied Psychology, 101: 1457-1473.

Zerres, A., Huffmeier, J., Freund, P. A., Backhaus, K. \& Hertel, G. 2013. Does it take two to tango? Longitudinal effects of unilateral and bilateral integrative negotiation training. Journal of Applied Psychology, 98: 478-491.

Zhan, Y., Wang, M. \& Shi, J. 2015. Retirees' motivational orientations and bridge employment: Testing the moderating role of gender. Journal of Applied Psychology, 100: 1319-1331.

Zhou, L., Wang, M., Chen, G. \& Shi, J. 2012. Supervisors' upward exchange relationships and subordinate outcomes: Testing the multilevel mediation role of empowerment. Journal of Applied Psychology, 97: 668-680. 
Zhu, L., Aquino, K. \& Vadera, A. K. 2016. What makes professors appear credible: The effect of demographic characteristics and ideological beliefs. Journal of Applied Psychology, 101: $862-880$.

\section{JOM}

Abbas, M., Raja, U., Darr, W. \& Bouckenooghe, D. 2014. Combined effects of perceived politics and psychological capital on job satisfaction, turnover intentions, and performance. Journal of Management, 40: 1813-1830.

Aryee, S., Walumbwa, F. O., Mondejar, R. \& Chu, C. W. L. 2017. Core self-evaluations and employee voice behavior: Test of a dual-motivational pathway. Journal of Management, 43: 946-966.

Bagger, J. \& Li, A. 2014. How does supervisory family support influence employees' attitudes and behaviors? A social exchange perspective. Journal of Management, 40: 1123-1150.

Bardolet, D., Brown, A. \& Lovallo, D. 2017. The effects of relative size, profitability, and growth on corporate capital allocations. Journal of Management, 43: 2469-2496.

Belschak, F. D., Hartog, D. N. D. \& Kalshoven, K. 2015. Leading machiavellians: How to translate machiavellians' selfishness into pro-organizational behavior. Journal of Management, 41: 1934-1956.

Beus, J. M. \& Whitman, D. S. 2017. Almighty dollar or root of all evil? Testing the effects of money on workplace behavior. Journal of Management, 43: 2147-2167.

Bizzi, L. 2013. The dark side of structural holes: A multilevel investigation. Journal of Management, 39: 1554-1578. 
Bordia, P., Restubog, S. L. D., Bordia, S. \& Tang, R. L. 2017. Effects of resource availability on social exchange relationships: The case of employee psychological contract obligations. Journal of Management, 43: 1447-1471.

Carlson, D. S., Hunter, E. M., Ferguson, M. \& Whitten, D. 2014. Work-family enrichment and satisfaction: Mediating processes and relative impact of originating and receiving domains. Journal of Management, 40: 845-865.

Chiaburu, D. S., Stoverink, A. C., Li, N. \& Zhang, X.-a. 2015. Extraverts engage in more interpersonal citizenship when motivated to impression manage: Getting along to get ahead? Journal of Management, 41: 2004-2031.

Collins, B. J. \& Mossholder, K. W. 2017. Fairness means more to some than others: Interactional fairness, job embeddedness, and discretionary work behaviors. Journal of Management, 43: $293-318$.

Dahling, J. J., Chau, S. L. \& O’Malley, A. 2012. Correlates and consequences of feedback orientation in organizations. Journal of Management, 38: 531-546.

Daniels, K., Beesley, N., Wimalasiri, V. \& Cheyne, A. 2013. Problem solving and well-being: Exploring the instrumental role of job control and social support. Journal of Management, 39: 1016-1043.

Den Hartog, D. N., Boon, C., Verburg, R. M. \& Croon, M. A. 2013. Hrm, communication, satisfaction, and perceived performance: A cross-level test. Journal of Management, 39: 1637-1665.

Dierdorff, E. C., Rubin, R. S. \& Bachrach, D. G. 2012. Role expectations as antecedents of citizenship and the moderating effects of work context. Journal of Management, 38: 573598. 
Dijke, M. v., Cremer, D. D., Brebels, L. \& Quaquebeke, N. V. 2015. Willing and able: Actionstate orientation and the relation between procedural justice and employee cooperation. Journal of Management, 41: 1982-2003.

Dillon, R. L., Tinsley, C. H., Madsen, P. M. \& Rogers, E. W. 2016. Organizational correctives for improving recognition of near-miss events. Journal of Management, 42: 671-697.

Ferris, D. L., Spence, J. R., Brown, D. J. \& Heller, D. 2012. Interpersonal injustice and workplace deviance: The role of esteem threat. Journal of Management, 38: 1788-1811.

González-Benito, J., Aguinis, H., Boyd, B. K. \& Suárez-González, I. 2012. Coming to consensus on strategic consensus: A mediated moderation model of consensus and performance. Journal of Management, 38: 1685-1714.

Greenbaum, R. L., Hill, A., Mawritz, M. B. \& Quade, M. J. 2017. Employee machiavellianism to unethical behavior: The role of abusive supervision as a trait activator. Journal of Management, 43: 585-609.

Greenbaum, R. L., Mawritz, M. B. \& Piccolo, R. F. 2015. When leaders fail to "walk the talk": Supervisor undermining and perceptions of leader hypocrisy. Journal of Management, 41: 929-956.

Harrison, J. S., Banks, G. C., Pollack, J. M., O’Boyle, E. H. \& Short, J. 2017. Publication bias in strategic management research. Journal of Management, 43: 400-425.

Heavey, C. \& Simsek, Z. 2017. Distributed cognition in top management teams and organizational ambidexterity: The influence of transactive memory systems. Journal of Management, 43: 919-945. 
Hochwarter, W. A., Ferris, G. R., Laird, M. D., Treadway, D. C. \& Gallagher, V. C. 2010. Nonlinear politics perceptions_-work outcomes relationships: A three-study, five-sample investigation. Journal of Management, 36: 740-763.

Hochwarter, W. A., Laird, M. D. \& Brouer, R. L. 2008. Board up the windows: The interactive effects of hurricane-induced job stress and perceived resources on work outcomes. Journal of Management, 34: 263-289.

Holtz, B. C. \& Harold, C. M. 2013. Interpersonal justice and deviance:The moderating effects of interpersonal justice values and justice orientation. Journal of Management, 39: 339-365.

Houwelingen, G. v., Dijke, M. v. \& Cremer, D. D. 2017. Fairness enactment as response to higher level unfairness:The roles of self-construal and spatial distance. Journal of Management, 43: 319-347.

Hüffmeier, J., Freund, P. A., Zerres, A., Backhaus, K. \& Hertel, G. 2014. Being tough or being nice? A meta-analysis on the impact of hard- and softline strategies in distributive negotiations. Journal of Management, 40: 866-892.

Kam, C., Morin, A. J. S., Meyer, J. P. \& Topolnytsky, L. 2016. Are commitment profiles stable and predictable? A latent transition analysis. Journal of Management, 42: 1462-1490.

King, E. B., Botsford, W., Hebl, M. R., Kazama, S., Dawson, J. F. \& Perkins, A. 2012. Benevolent sexism at work: Gender differences in the distribution of challenging developmental experiences. Journal of Management, 38: 1835-1866.

King, E. B., Mohr, J. J., Peddie, C. I., Jones, K. P. \& Kendra, M. 2017. Predictors of identity management: An exploratory experience-sampling study of lesbian, gay, and bisexual workers. Journal of Management, 43: 476-502. 
Kluemper, D. H., DeGroot, T. \& Choi, S. 2013. Emotion management ability: Predicting task performance, citizenship, and deviance. Journal of Management, 39: 878-905.

Kostopoulos, K. C., Spanos, Y. E. \& Prastacos, G. P. 2013. Structure and function of team learning emergence: A multilevel empirical validation. Journal of Management, 39: $1430-1461$.

Kukenberger, M. R., Mathieu, J. E. \& Ruddy, T. 2015. A cross-level test of empowerment and process influences on members' informal learning and team commitment. Journal of Management, 41: 987-1016.

Kunze, F., Jong, S. B. d. \& Bruch, H. 2016. Consequences of collective-focused leadership and differentiated individual-focused leadership: Development and testing of an organizational-level model. Journal of Management, 42: 886-914.

Lanzolla, G. \& Suarez, F. F. 2012. Closing the technology adoption-use divide: The role of contiguous user bandwagon. Journal of Management, 38: 836-859.

Lapierre, L. M. \& Allen, T. D. 2012. Control at work, control at home, and planning behavior: Implications for work-family conflict. Journal of Management, 38: 1500-1516.

Lee, Y.-T. \& Antonakis, J. 2014. When preference is not satisfied but the individual is: How power distance moderates person-job fit. Journal of Management, 40: 641-675.

Livingston, B. A., Schilpzand, P. \& Erez, A. 2017. Not what you expected to hear: Accented messages and their effect on choice. Journal of Management, 43: 804-833.

Madsen, P. M. 2013. Perils and profits: A reexamination of the link between profitability and safety in u.S. Aviation. Journal of Management, 39: 763-791. 
Marcus, B., Taylor, O. A., Hastings, S. E., Sturm, A. \& Weigelt, O. 2016. The structure of counterproductive work behavior: A review, a structural meta-analysis, and a primary study. Journal of Management, 42: 203-233.

McClelland, P. L., Liang, X. \& Vincent L. Barker, I. 2010. Ceo commitment to the status quo: Replication and extension using content analysis. Journal of Management, 36: 12511277.

Perry, S. J., Lorinkova, N. M., Hunter, E. M., Hubbard, A. \& McMahon, J. T. 2016. When does virtuality really "work"? Examining the role of work-family and virtuality in social loafing. Journal of Management, 42: 449-479.

Peters, K., Daniels, K., Hodgkinson, G. P. \& Haslam, S. A. 2014. Experts’ judgments of management journal quality: An identity concerns model. Journal of Management, 40: $1785-1812$.

Quinn, R. W. \& Bunderson, J. S. 2016. Could we huddle on this project? Participant learning in newsroom conversations. Journal of Management, 42: 386-418.

Restubog, S. L. D., Zagenczyk, T. J., Bordia, P., Bordia, S. \& Chapman, G. J. 2015. If you wrong us, shall we not revenge? Moderating roles of self-control and perceived aggressive work culture in predicting responses to psychological contract breach. Journal of Management, 41: 1132-1154.

Rosen, C. C., Slater, D. J., Chang, C.-H. \& Johnson, R. E. 2013. Let's make a deal: Development and validation of the ex post i-deals scale. Journal of Management, 39: 709-742.

Rosenbusch, N., Rauch, A. \& Bausch, A. 2013. The mediating role of entrepreneurial orientation in the task environment-performance relationship: A meta-analysis. Journal of Management, 39: 633-659. 
Shen, J. \& Benson, J. 2016. When csr is a social norm: How socially responsible human resource management affects employee work behavior. Journal of Management, 42: 1723-1746.

Steel, P., Kammeyer-Mueller, J. \& Paterson, T. A. 2015. Improving the meta-analytic assessment of effect size variance with an informed bayesian prior. Journal of Management, 41: 718-743.

Sue-Chan, C., Wood, R. E. \& Latham, G. P. 2012. Effect of a coach's regulatory focus and an individual's implicit person theory on individual performance. Journal of Management, 38: 809-835.

Treadway, D. C., Breland, J. W., Williams, L. M., Cho, J., Yang, J. \& Ferris, G. R. 2013. Social influence and interpersonal power in organizations: Roles of performance and political skill in two studies. Journal of Management, 39: 1529-1553.

Vagnani, G. 2015. Exploration and long-run organizational performance: The moderating role of technological interdependence. Journal of Management, 41: 1651-1676.

Vogel, R. M. \& Mitchell, M. S. 2017. The motivational effects of diminished self-esteem for employees who experience abusive supervision. Journal of Management, 43: 2218-2251.

Wallace, J. C., Butts, M. M., Johnson, P. D., Stevens, F. G. \& Smith, M. B. 2016. A multilevel model of employee innovation: Understanding the effects of regulatory focus, thriving, and employee involvement climate. Journal of Management, 42: 982-1004.

Wallace, J. C., Edwards, B. D., Paul, J., Burke, M., Christian, M. \& Eissa, G. 2016. Change the referent? A meta-analytic investigation of direct and referent-shift consensus models for organizational climate. Journal of Management, 42: 838-861. 
Way, S. A., Tracey, J. B., Fay, C. H., Wright, P. M., Snell, S. A., Chang, S. \& Gong, Y. 2015. Validation of a multidimensional hr flexibility measure. Journal of Management, 41: 1098-1131.

Wihler, A., Blickle, G., B. Parker Ellen, I., Hochwarter, W. A. \& Ferris, G. R. 2017. Personal initiative and job performance evaluations: Role of political skill in opportunity recognition and capitalization. Journal of Management, 43: 1388-1420.

Wu, C.-H. \& Parker, S. K. 2017. The role of leader support in facilitating proactive work behavior: A perspective from attachment theory. Journal of Management, 43: 1025-1049.

Wu, C.-H., Parker, S. K. \& Jong, J. P. J. d. 2014. Need for cognition as an antecedent of individual innovation behavior. Journal of Management, 40: 1511-1534.

Young, M. J., Morris, M. W. \& Scherwin, V. M. 2013. Managerial mystique: Magical thinking in judgments of managers' vision, charisma, and magnetism. Journal of Management, 39: 1044-1061.

Zagenczyk, T. J., Restubog, S. L. D., Kiewitz, C., Kiazad, K. \& Tang, R. L. 2014. Psychological contracts as a mediator between machiavellianism and employee citizenship and deviant behaviors. Journal of Management, 40: 1098-1122.

Zaheer, A., Castañer, X. \& Souder, D. 2013. Synergy sources, target autonomy, and integration in acquisitions. Journal of Management, 39: 604-632.

Zhang, X.-a., Li, N., Ullrich, J. \& Dick, R. v. 2015. Getting everyone on board: The effect of differentiated transformational leadership by ceos on top management team effectiveness and leader-rated firm performance. Journal of Management, 41: 1898-1933. 